\title{
Applied and Computational Mathematics Division
}

Summary of Activities for Fiscal Year 2012

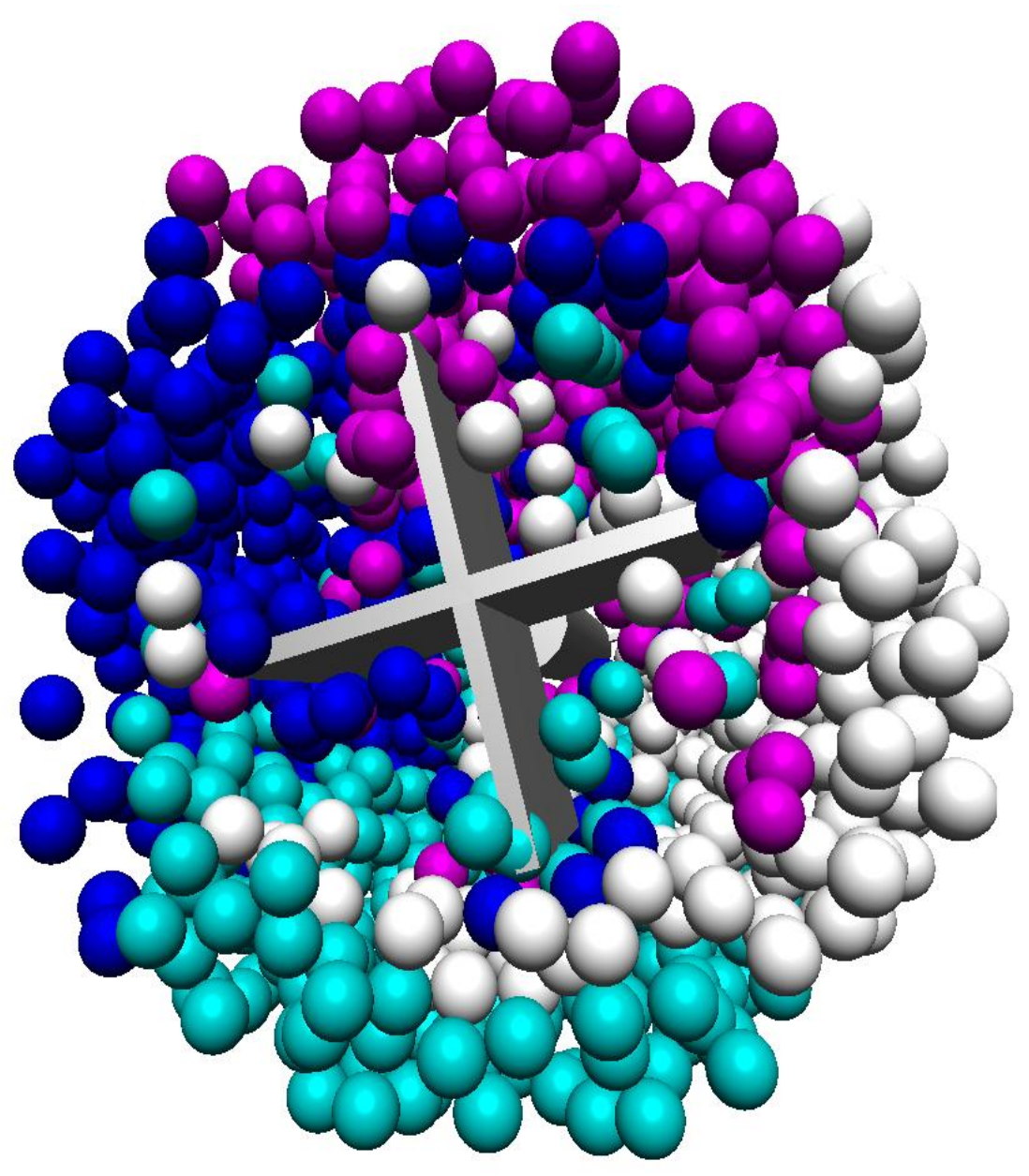

National Institute of Standards and Technology 

NISTIR 7931

\title{
Applied and Computational Mathematics Division
}

\section{Summary of Activities for Fiscal Year 2012}

\author{
Ronald F. Boisvert, Editor \\ Applied and Computational Mathematics Division \\ Information Technology Laboratory
}

http://dx.doi.org/10.6028/NIST.IR.7931

April 2013

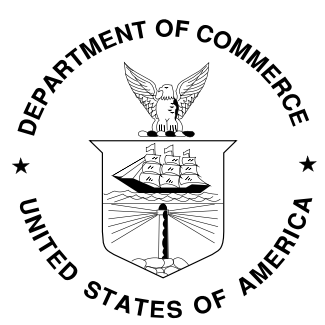

U.S. Department of Commerce

Rebecca Blank, Acting Secretary 



\section{$\underline{\text { Abstract }}$}

This report summarizes the technical work of the Applied and Computational Sciences Division of NIST's Information Technology Laboratory. Part I (Overview) provides a high-level overview of the Division's activities, including highlights of technical accomplishments during the previous year. Part II (Features) provides further details on ten projects of particular note this year. This is followed in Part III (Project Summaries) by brief synopses of all technical projects active during the past year. Part IV (Activity Data) provides listings of publications, technical talks, and other professional activities in which Division staff members have participated. The reporting period covered by this document is October 2011 through December 2012.

For further information, contact Ronald F. Boisvert, Mail Stop 8910, NIST, Gaithersburg, MD 208998910, phone 301-975-3812, email boisvert@nist.gov, or see the Division's web site at http://www.nist.gov/itl/math/index.cfm.

Cover Visualization: Snapshot from a simulation of a four-blade vane rheometer with a suspension of hard spheres. Each sphere is colored-coded by the rheometer octant in which it was located at the start of the simulation. The blades are rotating clockwise and have made approximately two full rotations at the time of this snapshot. For details, see page 28.

Acknowledgements: Thanks to Timothy Burns, Daniel Samarov, and Ginger White for their very careful reading of the manuscript.

Disclaimer: Certain commercial entities, equipment, or materials may be identified in this document in order to describe an experimental procedure or concept adequately. Such identification is not intended to imply recommendation or endorsement by the National Institute of Standards and Technology, nor is it intended to imply that the entities, materials, or equipment are necessarily the best available for the purpose. 


\section{Contents}

PART I: OVERVIEW

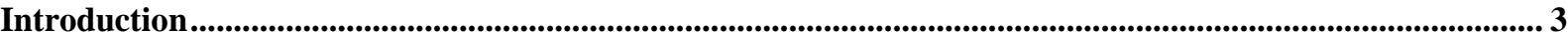

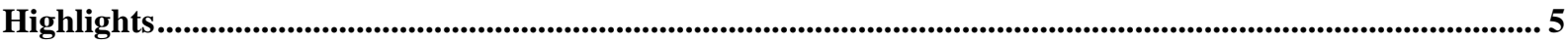

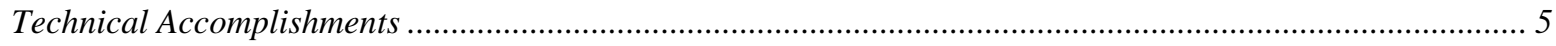

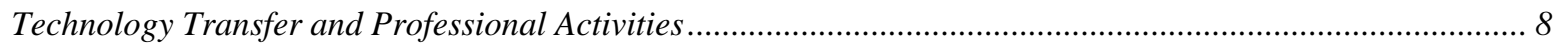

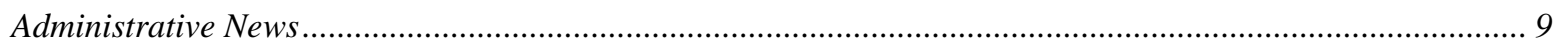

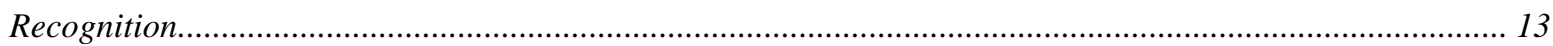

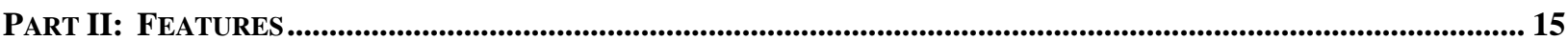

Quantum Algorithms for Quantum Field Theories ...................................................................................................... 17

Micromagnetic Investigation of Periodic Cross-Tie/Vortex Wall Geometry ....................................................... 19

Continuation Backward in Time in 2D Nonlinear Parabolic Equations, and an Experiment in Deblurring Nonlinearly Blurred Imagery............................................................................................................................ 22

Metrics for Manipulation and Enhancement of Forensic Images ........................................................................ 25

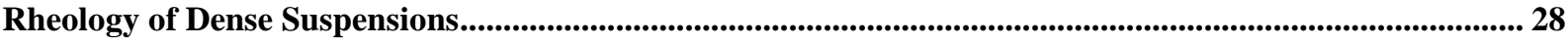

Visualization and Modeling of RF Propagation in Body Area Networks .............................................................. 32

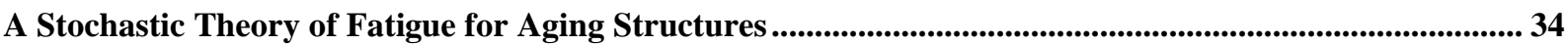

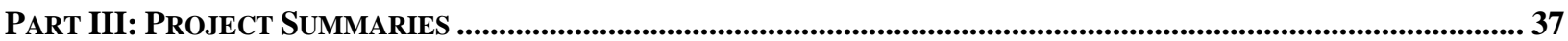

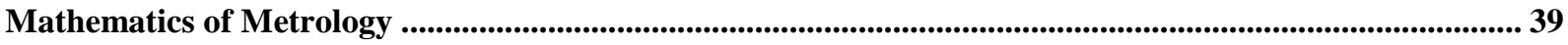

Continuation Backward in Time in 2D Nonlinear Parabolic Equations......................................................... 39

Micromagnetic Investigation of Periodic Cross-Tie/Vortex Wall Geometry ................................................... 39

Metrics for Manipulation and Enhancement of Forensic Images ............................................................... 39

A Stochastic Theory of Fatigue for Aging Structures .................................................................................. 39

Molecular Movies: Imaging Femtosecond Motion during Electrochemical Transitions ................................ 39

Virtual Measurements in Quantum Chemistry ..................................................................................... 41

Bubble Motion and Size Variation during Thermal Migration with Phase Change......................................... 41

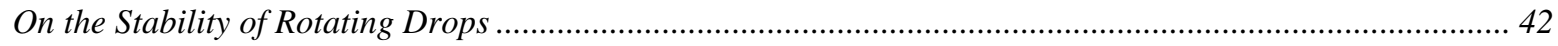

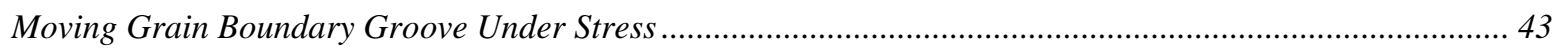

Measurement and Modeling of Plastic Flow Stress for High-Speed Machining Simulations ........................... 44

Selecting an Appropriate Statistical Distribution for a Class of Engineering Material Property Data............ 45

A Bayesian Nondestructive Evaluation Approach to Estimating Remaining Life of Aging Structures .............. 46

Simulation of Bioregulatory Networks Involved in Cell Cycle Control.......................................................... 47

Mathematical Optimization of Procedures for Cryoprotectant Equilibration ................................................. 48

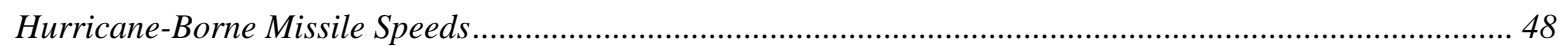

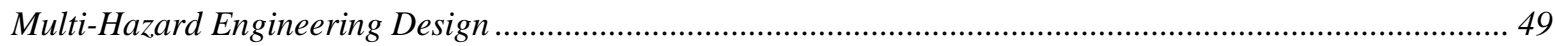

Alternative Methods of Latent Fingerprint Enhancement and Metrics for Comparison .................................. 50 


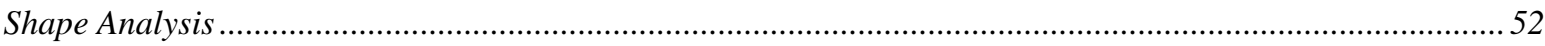

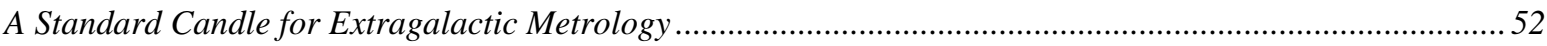

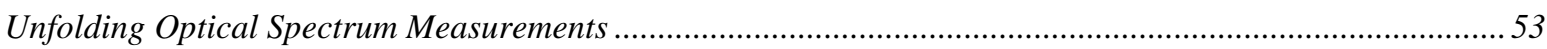

High Performance Computing and Visualization..................................................................................................................56

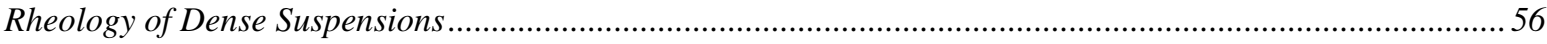

Visualization and Modeling of RF Propagation in Body Area Networks .....................................................56

Modeling and Visualization of Cement Paste Hydration and Microstructure Development ............................ 56

Nano-structures, Nano-optics, and How to Control Exciton Fine Structure with Electric Fields...................... 57

High Precision Calculations of Fundamental Properties of Few-Electron Atomic Systems ............................ 58

Determination of Computational Features to Quantitatively Characterize Stem Cell Colony Quality..............59

An Automated Method for Locating Phantom Nodules in Phantom CT Studies..............................................59

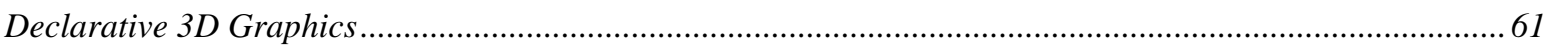

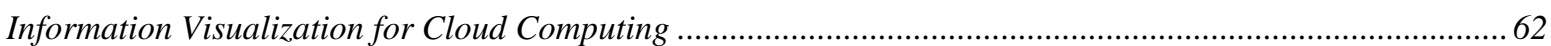

Interactive Visualization and Analysis Tools for the National Vulnerability Database .................................. 63

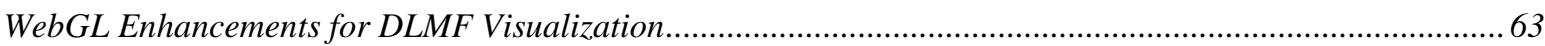

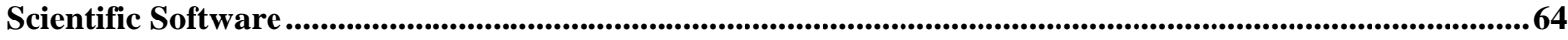

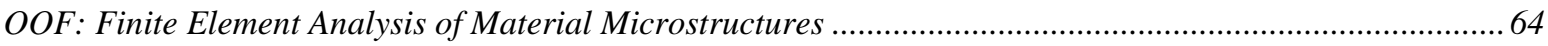

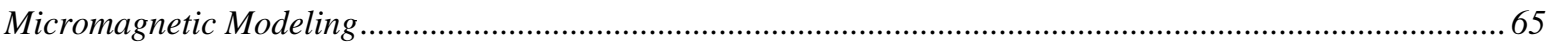

Parallel Adaptive Refinement and Multigrid Finite Element Methods ...........................................................66

Digital Library of Mathematical Functions ....................................................................................................................68

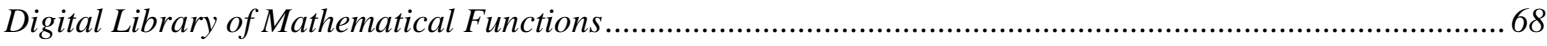

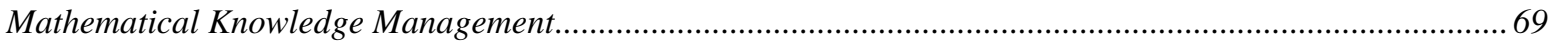

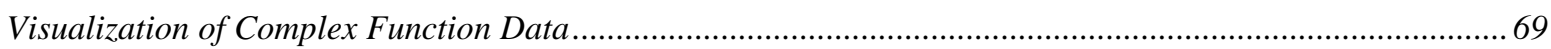

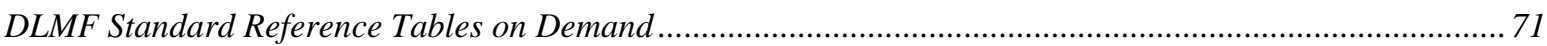

Special Function Expansions and Definite Integrals Associated with Fundamental Solutions of Linear Partial

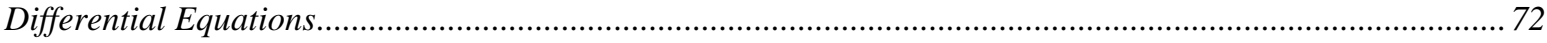

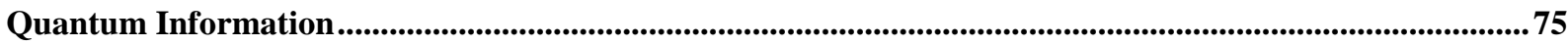

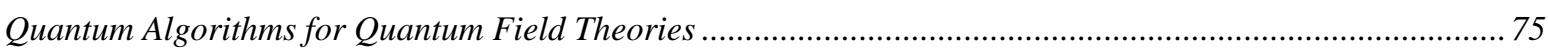

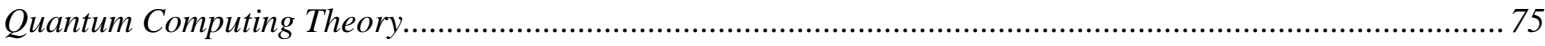

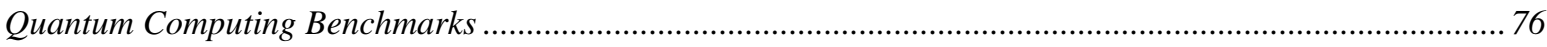

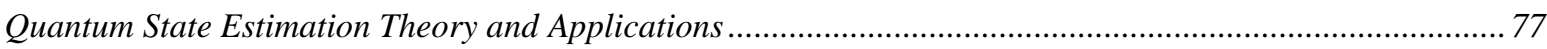

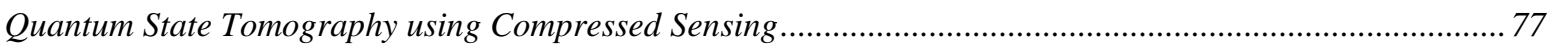

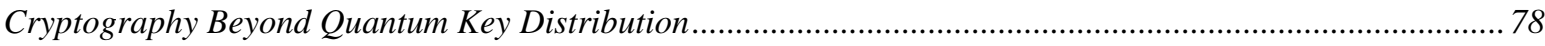

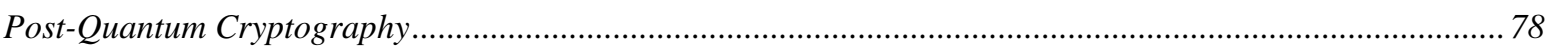

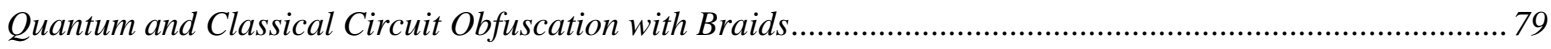

Generating Random Bits from Entangled Quantum States............................................................................ 79

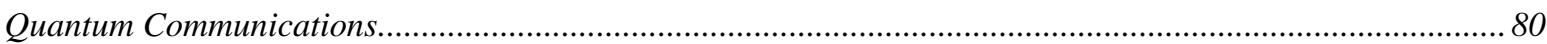

Low Density Parity Codes for Quantum Key Distribution........................................................................... 81

Foundations of Measurement Science for Information Systems ........................................................................82

A Game-theoretic Approach to the Security of Complex Systems................................................................... 82

An Algebraic Formulation for the Analysis and Visualization of Network Graphs ........................................ 83 


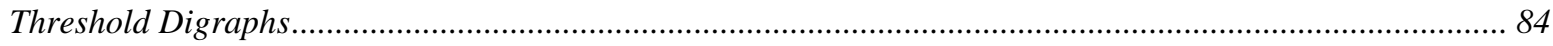

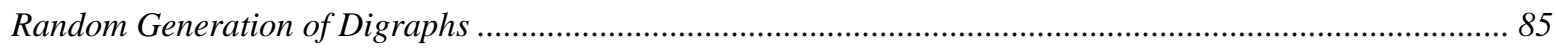

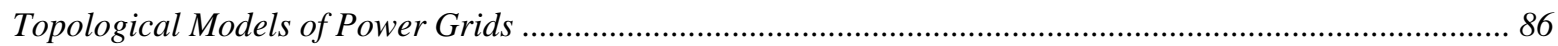

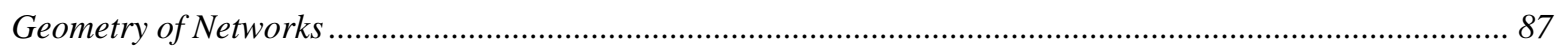

Broadcast and Spread of Information in Complex Networks ................................................................. 88

Security Risk Analysis of Enterprise Networks Using Attack Graphs ...................................................... 89

Distributed Motion Control in Mobile Sensor Networks .......................................................................... 90

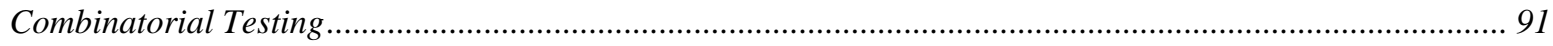

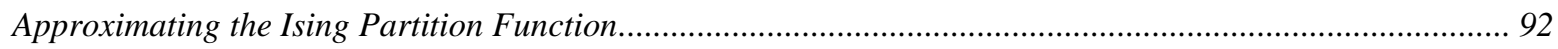

Spectral Algorithms for Document Topic Modeling ............................................................................ 93

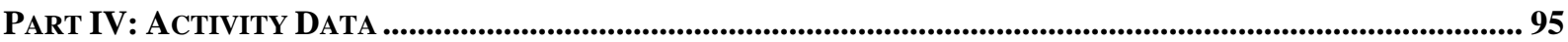

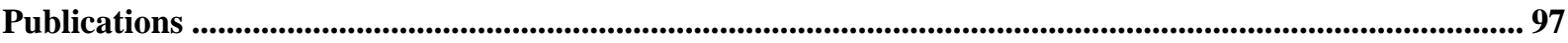

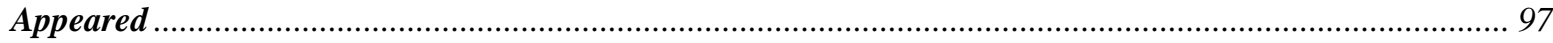

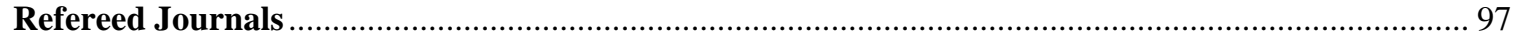

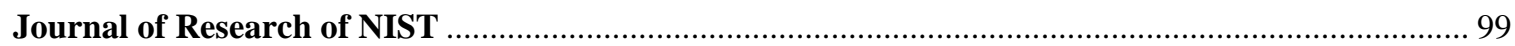

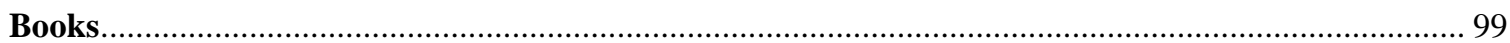

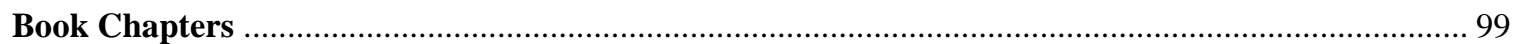

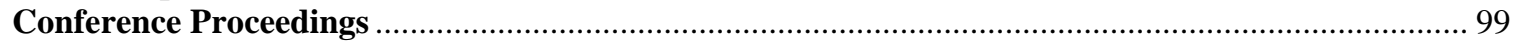

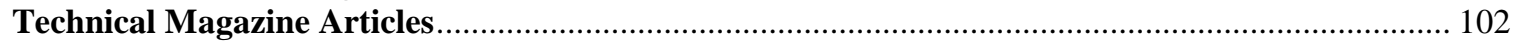

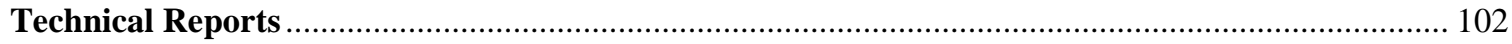

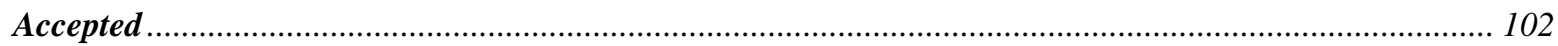

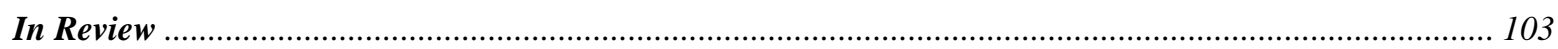

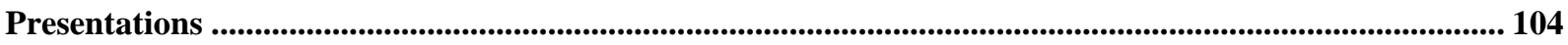

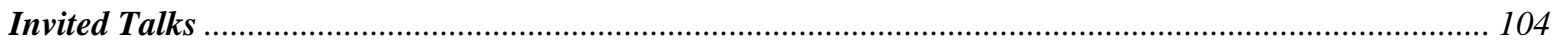

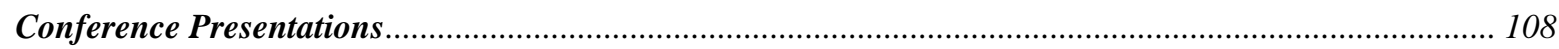

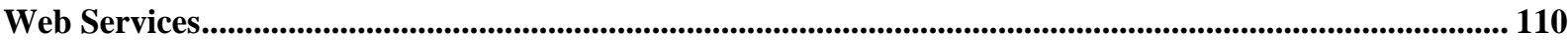

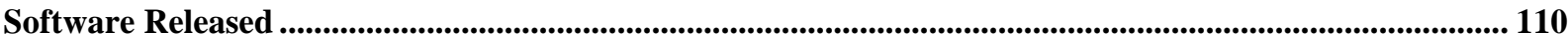

Conferences, Minisymposia, Lecture Series, Courses...................................................................................... 111

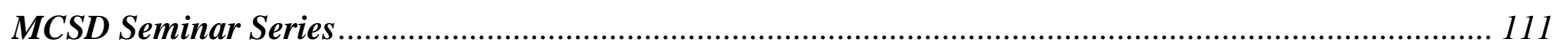

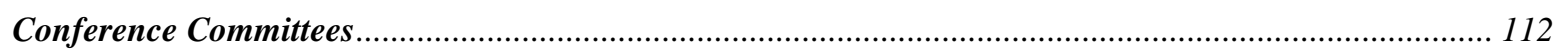

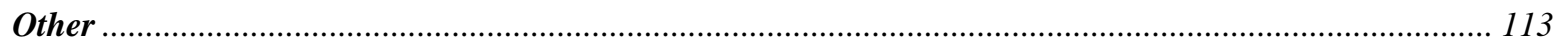

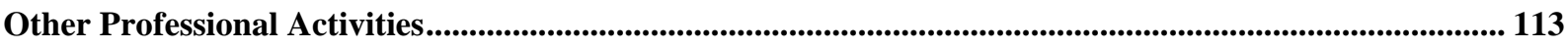

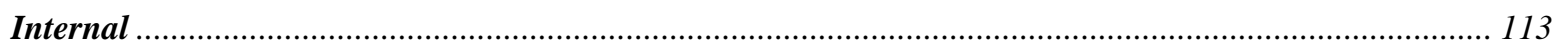

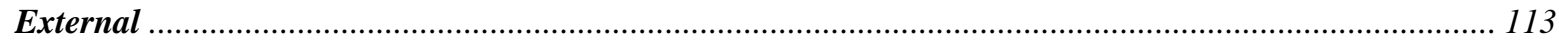

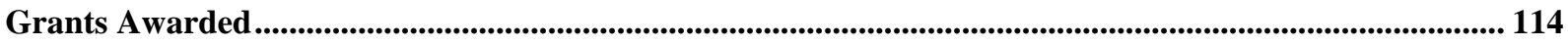

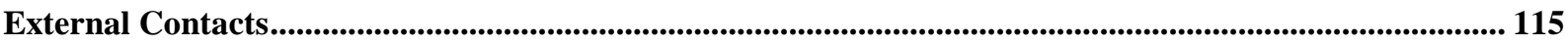

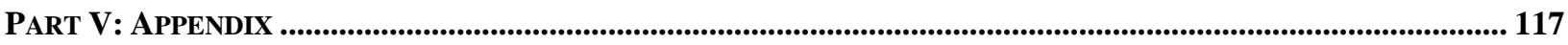

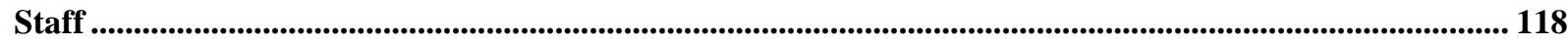

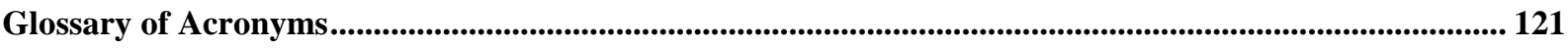




\section{Part I}

\section{Overview}

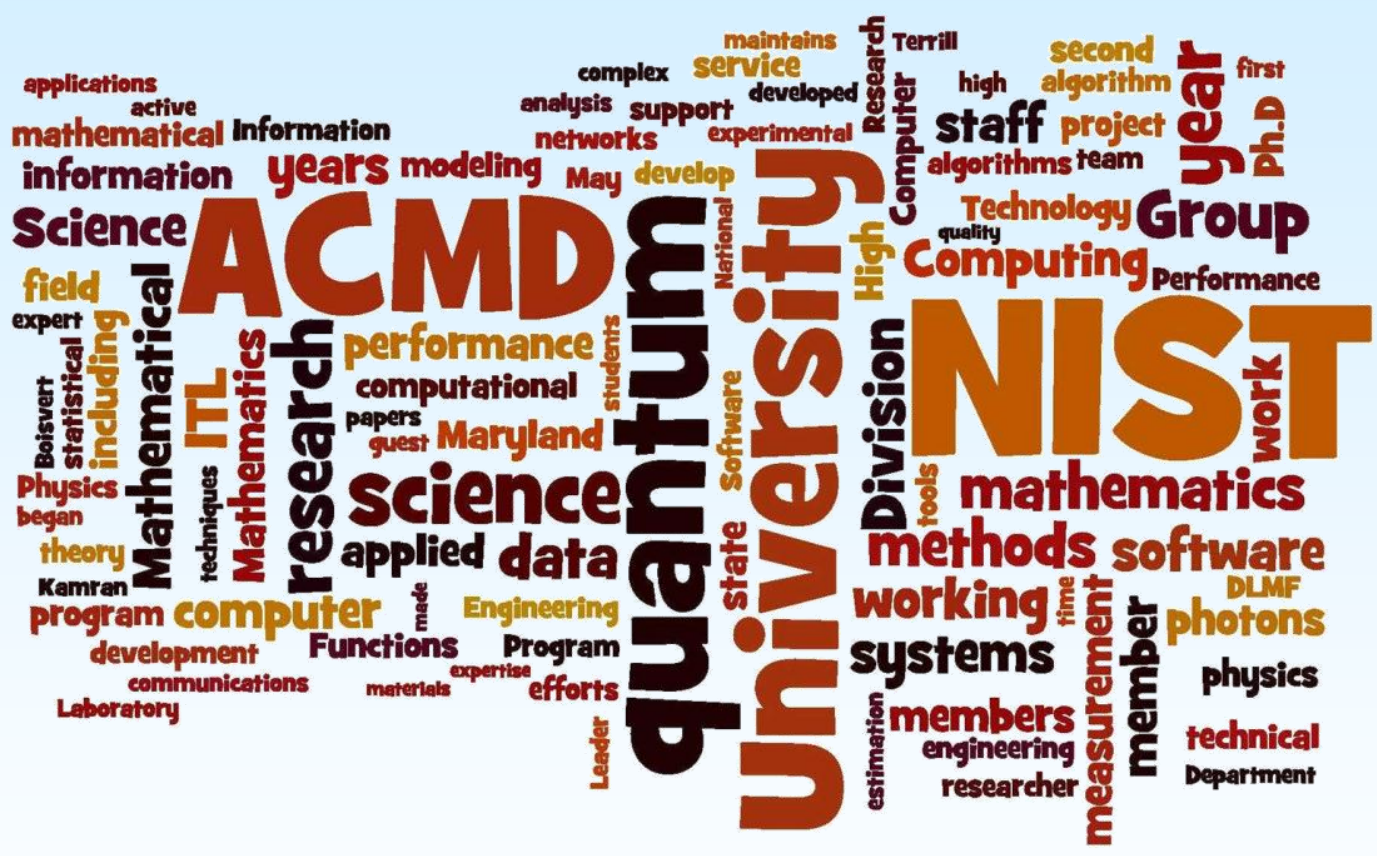




\section{Introduction}

Founded in 1901, NIST is a non-regulatory federal agency within the U.S. Department of Commerce. NIST's mission is to promote U.S. innovation and industrial competitiveness by advancing measurement science, standards, and technology in ways that enhance economic security and improve our quality of life. The NIST Laboratory program is broad-ranging, with research efforts in physics, electrical engineering, materials science, chemistry, bioscience, engineering, fire research, and information technology.

The Information Technology Laboratory (ITL) is one of six major laboratories and user facilities at NIST. ITL seeks to (a) accelerate the development and deployment of information and communication systems that are reliable, usable, interoperable, and secure; (b) advance measurement science through innovations in mathematics, statistics, and computer science; and (c) conduct research to develop the measurements and standards infrastructure for emerging information technologies and applications.

The Applied and Computational Mathematics Division (ACMD) is one of six technical Divisions in ITL. ACMD provides leadership within NIST in the use of applied and computational mathematics to solve science and engineering problems arising in measurement science and related applications. In that role ACMD staff members

- perform research and development in applied mathematics and computer science and engineering, including analytical methods, numerical and symbolic algorithms, advanced computing and communications architectures and applications, and high performance scientific visualization;

- engage in peer-to-peer collaborations in the application of mathematical and computational technologies to NIST problems;

- develop and disseminate mathematical reference data, software, and related tools; and

- work with internal groups and external organizations to develop standards, test procedures, reference implementations, and other measurement technologies for advanced scientific computation on current and future architectures.

Division staff is organized into four groups:

Mathematical Analysis and Modeling Group (Timothy Burns, Leader)

Performs research and maintains expertise in applied mathematics, mathematical modeling, and numerical analysis for application to measurement science.

Mathematical Software Group (Michael Donahue, Acting Leader)

Performs research and maintains expertise in the methodology and application of mathematical algorithms and software in support of computational science within NIST as well as in industry and academia.

Computing and Communications Theory Group (Ronald Boisvert, Acting Leader; Isabel Bechl and Xiao Tang, Project Leaders)

Performs research and maintains expertise in fundamental mathematics, physics, and measurement science necessary to enable the development of future computing and communications systems.

High Performance Computing and Visualization Group (Judith Terrill, Leader)

Performs research and maintains expertise in the methodologies and tools of high performance scientific computing and visualization for use in measurement science.

The technical work of the Division is organized into seven thematic areas; these are described in the sidebar. Six of these are represented by project descriptions in Section III of this document. The seventh, Virtual Measurements, is a cross-cutting program whose work is found under the Mathematics of Metrology, High Performance Computing and Visualization, and Scientific Software project descriptions. Projects that contribute to this crosscut note this fact in their descriptions in Section III. 


\section{Division Thematic Areas}

Mathematics of Metrology. Mathematics plays an important role in the science of metrology. Mathematical models are needed to understand how to design effective measurement systems, and to analyze the results they produce. Mathematical techniques are used to develop and analyze idealized models of physical phenomena to be measured, and mathematical algorithms are necessary to find optimal system parameters. Finally, mathematical and statistical techniques are needed to transform the resulting data into useful information. The goal of this work is to develop fundamental mathematical methods and analytical tools necessary for NIST to continue as a worldclass metrology institute, and to apply them to critical measurement science applications.

High Performance Computing and Visualization. Computational capability is advancing rapidly. This means that modeling and simulation can be done with greatly increased fidelity (e.g., higher resolution, more complex physics). However, developing large-scale parallel applications remains highly challenging, requiring expertise that application scientists rarely have. In addition, the hardware landscape is changing rapidly, so new algorithmic techniques must constantly be developed. We are developing and applying facilities and expertise of this type for application to NIST problems. Large scale computations and laboratory experiments invariably produce large volumes of scientific data, which cannot be readily comprehended without some form of visual analysis. We are developing the infrastructure necessary for advanced visualization of scientific data, including the use of 3D immersive environments and applying this to NIST problems. One of our goals is to develop the 3D immersive environment into a true interactive measurement laboratory.

Virtual Measurements. A virtual measurement is a quantitative result and its associated uncertainty, obtained by a computer simulation or computer-assisted measurements. We seek to develop metrology constructs, standard references, uncertainty characterization, and traceability into scientific computation and computerassisted measurements to enable predictive computing with quantified reliability.

Scientific Software. Modern science and engineering in general, and modern measurement science in particular, require a wide variety of software tools for scientific discovery, exploration, and analysis. As scientific inquiry becomes deeper and more specialized, so must the supporting software tools. The goal of this work is to develop critical software tools that support measurement science at NIST, as well as computational science and engineering at large.
Digital Library of Mathematical Functions. The special functions of applied mathematics are fundamental tools enabling modeling and analysis in all areas of science and engineering. To make effective use of such functions, practitioners must have ready access to a reliable source of information on their properties. The goal of this work is the development and dissemination of definitive reference data on the special functions of applied mathematics. The centerpiece of this effort is the DLMF, a freely available interactive and richly linked online resource.

Quantum Information. An emerging discipline at the intersection of physics and computer science, quantum information science (QIS) is likely to revolutionize science and technology in the same way that lasers, electronics, and computers did in the $20^{\text {th }}$ century. By encoding information into quantum states of matter, one can, in theory, exploit the seemingly strange and counterintuitive behavior of quantum systems to enable phenomenal increases in information storage and processing capability, as well as communication channels with extremely high levels of security. Although many of the necessary physical manipulations of quantum states have been demonstrated experimentally, scaling these up to enable fully capable quantum computers remains a grand challenge. We engage in (a) theoretical studies to understand the power of quantum computing, (b) collaborative efforts with the multi-laboratory experimental quantum science program at NIST to characterize and benchmark specific physical implementations of quantum information processing, and (c) the development and assessment of technologies for quantum communication.

Foundations of Measurement Science for Information Systems. Modern information systems are astounding in their complexity. Software applications are built from thousands of interacting components. Computer networks interconnect millions of independently operating nodes. Large-scale networked applications provide the basis for services of national scope, such as financial transactions and electrical power distribution. In spite of our increasing reliance on such systems, our ability to build far outpaces our ability to secure. Protocols controlling the behavior of individual nodes lead to unexpected macroscopic behavior. Local power anomalies propagate in unexpected ways leading to large-scale outages. Computer system vulnerabilities are exploited in viral attacks resulting in widespread loss of data and system availability. The long term stability of our critical infrastructure is simply unknown. Measurement science has long provided a basis for the understanding and control of physical systems. Similar types of deep understanding and insight are lacking for complex information systems. We seek to develop the mathematical foundations needed for a true measurement science for complex networked information systems. 


\section{Highlights}

In this section we identify some of the major accomplishments of the Division during the past year. We also provide news related to ACMD staff.

\section{Technical Accomplishments}

ACMD has made significant technical progress on many fronts during the past year. Here we highlight a few notable technical accomplishments with significant impact. Further details are provided in Part II (Features) and Part III (Project Summaries).

\section{Digital Library of Mathematical Functions}

The NIST Handbook of Mathematical Functions, published by Cambridge University Press in May 2010, has passed the 400 citation mark on Google Scholar ${ }^{1}$. The Handbook provides a succinct reference on the properties of the special functions of applied mathematics, which are a staple of mathematical modeling in many fields. Recent citations have come from published work on light scattering, instabilities in rocks, cosmology, elementary quantum systems, quantum field theory, general relativity, statistical mechanics, nanophotonics and search algorithms. The NIST Digital Library of Mathematical Functions $^{2}$ (DLMF), a parallel free online resource, has satisfied nearly 5 million full page requests resulting from more than 615,000 user sessions over the same 32 month period. The main goal of the DLMF project, conceived nearly 15 years ago and carried out by ITL and PML, was to develop a $21^{\text {st }}$ century successor to the legendary Handbook of Mathematical Functions, a classic 1964 NBS publication edited by Milton Abramowitz and Irene Stegun (A\&S). The most cited publication in NIST history, A\&S still holds the lead in citations, with more than 60,000 recorded on Google Scholar, albeit with nearly a 50-year head start over DLMF. Together, A\&S and DLMF present vibrant faces of NIST to the technical community, one of great heritage, the other looking forward. [See page 68.]

\section{Quantum Information}

Stephen Jordan of ACMD, working with colleagues at Caltech and the University of Pittsburgh, has developed the first quantum algorithm for efficiently simulating a quantum field theory. The paper describing this work was published as a feature article in Science on June 1,2012. The significance of the achievement was explained in an associated Perspectives article by Hauke, Tagliacozzo and Lewenstein ${ }^{3}$. Quantum field theories are used to describe systems in which the effects of quantum mechanics and special relativity are both important. The Standard Model of particle physics, which aims to explain all physical phenomena excluding gravity, is a quantum field theory. Calculating quantitative predictions from a quantum field theory is extremely difficult. In particular, no efficient algorithm is known for classical computers to simulate the dynamics of quantum field theories involving strongly-interacting particles. Jordan and colleagues devised a quantum algorithm to simulate the simplest nontrivial quantum field theory, massive phi-fourth $\left(\varphi^{4}\right)$ theory. Their algorithm calculates scattering probabilities in relativistic particle collisions similar to those that occur in particle accelerators like the Large Hadron Collider. The algorithm scales in time polynomially in the number of particles, their energy, and the desired precision, and works even when the coupling between particles is strong. By studying whether quantum computers can efficiently simulate the Standard Model, Jordan and colleagues also hope to shed light on the question of the fundamental computational power of the universe, by either confirming or refuting the oft-repeated claim that the universe is equivalent in computational power to a quantum computer. [See page 17.]

NIST has developed a robust experimental program in quantum-based masurements ${ }^{4}$. A fundamental problem in this area is how to fully characterize a quantum state created in a laboratory.

\footnotetext{
${ }^{1}$ http://scholar.google.com/

2 http://dlmf.nist.gov/

3 P. Hauke, L. Tagliacozzo and M. Lewenstein, Speeding Up Quantum Field Theories, Science 336 (June 1, 2012 ), $1122-1123$.

${ }^{4}$ http://www.nist.gov/public_affairs/factsheet/quantum_meas_2010.cfm
} 
Unfortunately, no single measurement can completely characterize a quantum state. Furthermore, any single measurement is, in fact, destructive. Thus, quantum state estimation is a statistical problem of estimating an underlying quantum state using a collection of measurements made on independently prepared copies of the state. We are working to develop improved mathematical techniques for doing this. Two major efforts have been undertaken. Scott Glancy and Manny Knill are working to ameliorate well-known biases in traditional methods of quantum state estimation, applying the resulting techniques to the characterization of quantum states produced in PML. Yi-Kai Liu is exploiting methods of compressed sensing to significantly reduce the experimental and computational burden on state estimation through greatly improved sampling techniques. [See page 77.]

Tests performed by Paulina Kuo, Oliver Slattery, and Xiao Tang of ACMD show that a prototype device developed with collaborators at Stanford University can significantly speed up quantum information transfer through fiber-optic cables ${ }^{5}$. Conventional fiber-optic systems, in use for decades, transmit data as a series of light pulses. Such pulse streams can be intercepted by third parties undetectably. But individual photons themselves can carry data, encoded in their quantum states. Because any attempt to intercept that data alters the quantum state, eavesdroppers can always be detected. This property is the basis for the security of quantum key distribution systems. While information scientists have found a way to encode photon quantum states successfully, practical systems need the photons to be at wavelengths compatible with both existing optical-fiber networks and single-photon sensitive (and economically viable) silicon detectors. Unfortunately, these wavelengths are different. One potential solution is to change the photons' wavelength from the infrared - desirable for fiber networks - to the visible spectrum, so that a silicon detector can "see" them. A method of doing so is to mix the information-carrying photons with a second photon beam. The information-carrying photons absorb this second beam's additional energy and get kicked up from the infrared region of the spectrum to the wavelength of visible red light, which silicon sensors can detect. However, silicon single-photon detectors cannot operate very fast, which puts limitations on data rates and ultimately, their usefulness for quantum information transmission systems. The heart of the newly developed device is a new crystal that goes beyond converting the wavelength of the photons. Designed and fabricated by Stanford's Jason Pelc, the crystal is capable of splitting the beam of infrared, information-carrying photons into two distinct beams of slightly different color, and directing the different-colored photons to different outputs. Controlling the flow to either output allows the team to use two "slow" detectors in place of one, thereby doubling the overall system speed. ACMD tests showed that this innovation allows twice as much data to be sent in a single beam. Since the photons conceivably can be split not just into two, but several different beams, this provides a promising solution to the detector problem. [See page 80.]

\section{Scientific Software}

This year ACMD's software system, $\mathrm{PHAML}^{6}$, for parallel solution of elliptic partial differential equations (PDEs) based on adaptive grid refinement and multigrid methods, was used as a platform for a major survey of methods for $h p$ adaptive grid refinement. Adaptive methods, which automatically make localized adjustments to the method to optimize use of computational resources, are essential for successfully attacking the most challenging modeling problems based on PDEs. $h$-based methods adapt by refining in space (i.e., reducing size of triangles in the computational grid), while $p$-based methods adapt by increasing the polynomial order of approximation on fixed-size grid elements. $h p$ methods combine the two. Many heuristics have been proposed for implementing such a refinement strategy. 13 separate strategies were evaluated on a battery of 12 test problems. Not only do the results provide useful data on the comparative performance of the methods, but they also provide insight on practical tradeoffs between efficiency and performance. The work was carried out by William Mitchell and Marjorie McClain. [See page 66.]

\footnotetext{
${ }^{5}$ This description is adapted from an article in NIST TechBeat.

${ }^{6}$ PHAML stands for Parallel Hierarchical Adaptive Multi-Level; see http://math.nist.gov/phaml/
} 


\section{High Performance Computing and Visualization}

A team of researchers from ACMD and EL were awarded 22 million hours of computer time in 2012 from the Department of Energy to support the study of the flow properties of large-particle suspensions such as concrete ${ }^{7}$. The award is for the second year of a three year, peer reviewed, proposal to DOE's Innovative and Novel Computational Impact on Theory and Experiment (INCITE) program ${ }^{8}$. High fidelity flow simulations with many thousands of particles, with a wide range of sizes and shapes in a non-Newtonian fluid matrix, are enabling the determination of fundamental rheological parameters such as stress and strain rate in non-analytical rheometers and mixing geometries, properties that cannot now be measured accurately in industrial settings. NIST standard reference materials for suspension rheology are also being designed using the results of these simulations. Not only is this work solving a critical outstanding problem in the cement and concrete industry, but it is expected to have an enormous influence on the wide array of industries that use vane rheometers and mixers such as food processing, water treatment, coatings, and pharmaceuticals. The research team includes William George, Marc Olano, and Judith Terrill of ACMD, Nicos Martys and Edward Garboczi of the NIST Engineering Laboratory, and Pascal Hebraud of CNRS/ESPCI (France). Simulations are being run in the Leadership Computing Facility of Argonne National Laboratory on "Intrepid," an IBM Blue Gene/P system with 164,000 cores, 80 terabytes of RAM, and a peak performance of 557 teraflops. [See page 28.]

ACMD staff members are also contributing to the improvement of medical imaging technology. The Quantitative Imaging (QI-Bench) group is a NIST-sponsored organization developing "open-source informatics tooling used to characterize the performance of quantitative medical imaging as needed to advance the field". QI-Bench maintains a publicly available FDA database consisting of more than 1,000 CT scans of artificial lungs embedded with "phantom" tumors intended for facilitating the assessment of lung cancer nodule size estimation methodologies and tools. The use of these scans would be greatly facilitated by the availability of phantom nodule location data ("ground truth"). This data is currently unavailable, and generating it through laborious inspections of trained radiologists is prohibitively time consuming and expensive. Adele Peskin of ACMD has written and tested an initial version of software to perform automatic lung cancer nodule location. For data sets with thin slice distances $(0.4 \mathrm{~mm}$ vs. 1.25 $\mathrm{mm}$ ), her algorithm accurately locates over $93 \%$ of the nodules as identified by subject matter experts, with a false positive rate of $3.5 \%$. Preliminary results were presented this year at a peer-reviewed conference. The software is currently being enhanced to extend this NIST-developed analysis to the 1,187 sets of CT scans of FDA phantoms on the QI-bench website. [See page 59.]

\section{Foundations of Measurement Science for Information Systems}

On June 8, 2012 ACMD hosted a Workshop on Large-scale Complex Networks aimed at highlighting and discussing current research on: (a) large-scale geometry of complex networks, including hyperbolicity and its possible impact on network performance, reliability, robustness, and security; (b) processes on complex networks, including percolation and diffusion; and (c) interplay between processes on networks and network evolution. Some 30 people participated, including researchers from institutions such as the Army Research Lab, Bell Labs, Stanford University, Stony Brook University, the University of Illinois, the University of Maryland, and the University of Southern California. This was the second of a series of workshops on this topic co-sponsored by Bell Labs and NIST. Vladimir Marbukh and Assane Gueye organized the event.

This year we also established a new external partnership that will allow us to engage the complex systems research community on topics of interest to ACMD. Using grant funding from NIST, the Santa Fe Institute (SFI) will organize a series of small working groups, larger workshops, and collaborative visits to and from SFI in the areas of network science, biologically inspired computation, and computer security. The first such workshop, entitled Structure, Statistical Inference, and Dynamics in Networks: From Graphs to Rich Data will be held in Santa Fe in May 2013. That workshop will consider how net-

\footnotetext{
${ }^{7}$ http://www.alcf.anl.gov/articles/us-department-energy-awards-alcf-resources-researchers-addressing-global-science-issues

${ }^{8}$ http://www.science.energy.gov/news/in-focus/2011/11-15-11-2/

9 http://www.qi-bench.org/
} 
work data beyond simple connectivity can be captured and used to obtain new understanding of complex interconnected systems. The second, entitled Deep Computation in Statistical Physics, to be held in the summer of 2013, will consider how novel algorithms and methods of statistical physics can contribute to the understanding of complex systems more broadly.

\section{Mathematics of Metrology}

Fingerprint evidence collected at crime scenes is rarely pristine. The images are incomplete, suffer from pressure gradations, are partially obscured by underlying surface patterns and colors, etc. Due to the low signal quality of the fingerprint such images are often not suitable for input into fingerprint identification software to search for matches. Because of this, forensic scientists currently use a variety of image processing tools to improve the quality of these images. To date, such latent fingerprint image enhancement has been treated as a preprocessing step for subsequent identity matching analysis. As dramatic and essential as these transformations are, no research exists to characterize this step of the evidentiary analysis chain. Lack of such characterizations can result in detrimental consequences for reproducibility, traceability, and quantification of accuracy. This year Andrew Dienstfrey and Alfred Carasso of ACMD, in collaboration with a larger NIST team, have undertaken a first investigation of this step of the forensic analysis workflow. [See page 39.]

Finally, this year we also established a formal partnership with the Institute for Mathematics and Its Applications (IMA) at the University of Minnesota for work associated with the Materials Genome Initiative. The IMA project will study molecular dynamics (MD) modeling as used to predict the material properties of resins. Resins are a critical component of composite materials such as those used in the development of the Boeing 787 Dreamliner. Of particular interest is the development of techniques and tools to quantify the uncertainty in the results of such MD simulations. The work will be a collaboration between the IMA, Boeing, and NIST. To facilitate the collaboration, the IMA will identify an industrial postdoctoral fellow who will spend time at each institution.

\section{Technology Transfer and Professional Activities}

The volume of technical output of ACMD remains high. During the last 18 months, Division staff members were (co-)authors of 51 articles appearing in peer-reviewed journals, 37 papers in conference proceedings, and 7 magazine articles. Sixteen additional papers have been accepted for publication, while 22 others are undergoing review. Division staff members gave 93 invited technical talks and presented 48 others in conferences and workshops.

ACMD continues to maintain an active Web site with a variety of information and services, including the Digital Library of Mathematical Functions, the Guide to Available Mathematical Software, the Matrix Market, and the SciMark Java benchmark. During calendar year (CY) 2012, the division web server satisfied more than 4.4 million requests for pages from more than 370,000 distinct hosts. In total, there have been more than 326 million "hits" on ACMD Web servers since they went online as NIST's first web servers in 1994. The individual software packages that we produce and distribute also continue to see very high usage. Table 1 lists download counts for some of our most popular ones. Another indication of the successful transfer of our technology is references to our software in refereed journal articles. For example, our OOMMF software for nano-magnetic modeling was cited in 148 such papers which were published in CY 2012 alone (more than 1,440 such papers have been identified since 2001).

Members of the Division are also active in professional circles. Staff members hold a total of 15 editorial positions in peer-reviewed journals. This year Isabel Beichl completed her second term as Editorin-Chief of the IEEE/AIP publication Computing in Science \& Engineering, and ACMD faculty appointee Dianne O'Leary serves at Editor-in-Chief of the SIAM Journal on Matrix Analysis and Applications. Staff members are also active in conference organization, serving on 18 organizing/steering/program committees.

Service within professional societies is also prevalent among our staff. For example, Ronald Boisvert serves as Co-Chair of the Publications Board of the Association for Computing Machinery (ACM) and is a member of the ACM Council, the association's board of directors. In addition, staff members 
Table 1. Downloads of Selected Division Software Packages During CY 2012

\begin{tabular}{llr}
\hline Package & \multicolumn{1}{c}{ Description } & Downloads \\
\hline JAMA & Linear algebra in Java & 27,426 \\
\hline OOMMF & Modeling of nano-magnetic phenomena & 5,000 \\
\hline TNT & Linear algebra using C++ templates & 3,890 \\
\hline OOF & Modeling materials with complex microstructure & 1,217 \\
\hline f90gl & Fortran 90 interface to OpenGL graphics & 1,162 \\
\hline spblas & Sparse basic linear algebra components & 1,131 \\
\hline Ngraph & Basic graph algorithms; web crawler & 812 \\
\hline PHAML & Parallel adaptive solution of partial differential equations & 518 \\
\hline
\end{tabular}

serve on committees of the IEEE Computer Society and the Mathematical Association of America. Staff members are also active in a variety of working groups. For example, Ronald Boisvert completed 10 years of service as Chair of the International Federation for Information Processing (IFIP) Working Group 2.5 on Numerical Software, Donald Porter is a member of the Tcl Core Team, Bruce Miller is a member of W3C's Math Working Group, Kamran Sayrafian is a member of the IEEE 802.15 Working Group on Wireless Personal Area Networks, and Sandy Ressler is a member of the Declarative 3D for the Web Architecture Community Group. Judith Terrill represents NIST on the High End Computing Interagency Working Group of the Federal Networking and Information Technology Research and Development (NITRD) Program. Robert Bohn is Co-Chair of the NITRD Program's Faster Administration of Science and Technology Education and Research (FASTER) Community of Practice.

For further details, see Section IV, Activity Data.

\section{Administrative News}

The past year saw many staffing changes. Among these are the following.

\section{$\underline{\text { Arrivals }}$}

Kamran Sayrafian joined ACMD after four years of service as the ITL Program Manager for Pervasive Information Technology. Kamran holds Ph.D., M.S. and B.S. degrees in Electrical and Computer Engineering from University of Maryland, Villanova University and Sharif University of Technology, respectively. He has been at NIST since 2004. Prior to joining NIST, he was the cofounder of Zagros Networks, Inc., a fabless semiconductor company based in Rockville, MD where he served as President and senior member of the architecture team. Kamran is the co-inventor of four U.S. patents. He is a senior member of IEEE and an adjunct faculty of the University of Maryland. While an ITL Program Manager, Kamran started up and participated in several research efforts in collaboration with ACMD, including studies of RF propagation in body area networks and in the optimal deployment of mobile sensors.

An expert in computer graphics, Wesley Griffin joined ACMD's permanent staff in the fall of 2012 as part of the High Performance Computing and Visualization Group. Wesley received a B.S. degree from the University of Maryland, and an M.S. from UMBC, both in Computer Science. He is presently a Ph.D. candidate at UMBC, working under the direction of ACMD faculty appointee Marc Olano on variable bit rate texture compression, real-time decompression, and objective color image quality assessment.

Amanda Streib and Noah Streib began two-year appointments in ACMD as NIST NRC Postdoctoral Associates in July 2012. Amanda received a B.S. in mathematics from Furman University in 2006 and a Ph.D. from Georgia Tech's program in Algorithms, Combinatorics and Optimization in May 2012. Her thesis studied mixing rates of Markov chains with applications in computer science and statistical physics. She was a National Physical Sciences Consortium Fellow at Georgia Tech. Noah also graduated with a $\mathrm{Ph} . \mathrm{D}$. from the same program at Georgia Tech. He received a B.A. in mathematics from Oberlin College in 2006. At Georgia Tech he studied the combinatorics of finite partially ordered sets, completing a thesis 
on planar and Hamiltonian cover graphs. At NIST Amanda and Noah are working with Isabel Beichl on approximations of the partition function for the Ising model of statistical physics.

Recipient of an NSF Postdoctoral Fellowship for Transformative Computational Science using CyberInfrastructure, Daniel Kaslovsky joined ACMD in Boulder in the fall of 2012 as a guest researcher. Daniel received a B.A. in computer science from Colgate University in 2003 and a Ph.D. in Applied Mathematics from the University of Colorado in Boulder in May 2012. At NIST he will be working with Brad Alpert on the development of computational algorithms based on random sampling for efficient processing of compressible data.

Catherine Graham joined ACMD as Division Secretary in February 2012. A NIST staff member since 2000, Cathy had previous assignments in the NIST Manufacturing Engineering Laboratory and the NIST Baldridge Performance Excellence Program.

Paulina Kuo of the Joint Quantum Institute (a cooperative venture of NIST and the University of Maryland) joined ACMD in October 2011 as a Guest Researcher. Paulina received undergraduate degrees in Physics and Materials Science from MIT. In 2008 she received a PhD in Applied Physics from Stanford University. She also served as an NRC Postdoctoral Associate in PML. An expert in nonlinear optics, Paulina will be contributing to the Division's experimental efforts in quantum communications.

Ismet Sahin began a term in ACMD as an ARRA Senior Fellow in October 2011. Sahin has a PhD in Electrical and Computer Engineering from the University of Pittsburgh (2006). He was most recently a Guest Researcher in the NIST National Center for Neutron Research. Ismet will be working with Anthony Kearsley on optimization methods applied to waveform time delay estimation algorithms that are used in seismology, radar, sonar, and biomedical science.

Yong-Su Kim joined ACMD in March 2012 as a guest researcher from the Pohang University of Science and Technology (POSTECH) in South Korea. An expert in quantum optics, Yong-Su is contributing to ITL's quantum communications project, especially in experimental techniques for the conversion of flying qubits to stationary qubits.

Itzel Dominquez Mendoza of the Centro Nacional de Metrología (CENAM), NIST's sister institution from Mexico began an appointment as a guest researcher in ACMD in September 2012. Participating in the ITL project on combinatorial software testing, she is working on the inclusion of constraints in the team's coverage measurement tool.

Santanu Sarkar, a recent Ph.D. in Mathematics from the Indian Statistical Institute joined ACMD as a guest researcher in late 2012. An expert on cryptanalysis, Santanu is working with the cryptography group from ITL's Computer Security Division on number-theoretic tools for improving performance and/or functionality of cryptographic applications.

\section{Departures}

Daniel Lozier retired from NIST in February 2012 after 47 years of Federal service. Dan's government career began in 1963 at the Army R\&D Lab at Ft. Belvoir. He joined the National Bureau of Standards in 1969 after completing a Ph.D. in applied mathematics from the University of Maryland. An expert in the computation of special functions, Dan held many leadership positions during his long tenure at NIST including Chair of the SIAM Activity Group on Orthogonal Polynomials and Special Functions, President of the NIST Chapter of Sigma Xi, Associate Editor of the Mathematics of Computation, and Leader of the NIST Mathematical Software Group. Dan's most significant contribution was as project lead for the NIST Digital Library of Mathematical Functions (DLMF), for which he earned a Department of Commerce Gold Medal in 2011. In the spring of 2012 Dan returned to NIST to continue his contributions to the DLMF project on a part-time basis.

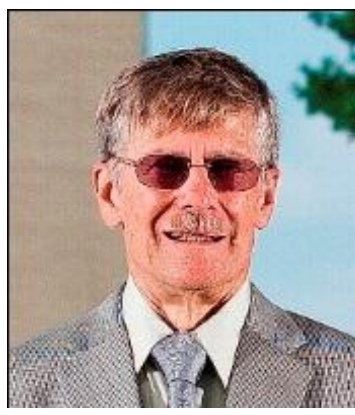

Figure 1. Daniel Lozier. 
Table 2. Student Interns in ACMD

\begin{tabular}{|c|c|c|c|c|c|}
\hline Name & Institution & Level & Program & Mentor & Project Title \\
\hline $\begin{array}{l}\text { Styvens } \\
\text { Belloge }\end{array}$ & $\begin{array}{l}\text { ISIMA } \\
\text { (France) }\end{array}$ & G & $\begin{array}{l}\text { Guest } \\
\text { Researcher }\end{array}$ & J. Terrill & $\begin{array}{l}\text { Visualization and analysis of the } \mathrm{Na}- \\
\text { tional Vulnerability Database }\end{array}$ \\
\hline $\begin{array}{l}\text { Hamza } \\
\text { Attak }\end{array}$ & $\begin{array}{l}\text { ISIMA } \\
\text { (France) }\end{array}$ & G & $\begin{array}{l}\text { Guest } \\
\text { Researcher }\end{array}$ & J. Terrill & Visualization algorithms \\
\hline $\begin{array}{l}\text { Gregory } \\
\text { Rami }\end{array}$ & $\begin{array}{l}\begin{array}{l}\text { ISIMA } \\
\text { (France) }\end{array} \\
\end{array}$ & G & $\begin{array}{l}\text { Guest } \\
\text { Researcher }\end{array}$ & J. Terrill & Impact of fire on building structures \\
\hline $\begin{array}{l}\text { Martina } \\
\text { Barbi } \\
\end{array}$ & $\begin{array}{l}\text { University } \\
\text { of Bologna }\end{array}$ & G & $\begin{array}{l}\text { Guest } \\
\text { Researcher }\end{array}$ & K. Sayrafian & RF propagation in body-area networks \\
\hline $\begin{array}{l}\text { Deyan } \\
\text { Ginev }\end{array}$ & $\begin{array}{l}\text { Jacobs } \\
\text { University } \\
\text { (Germany) }\end{array}$ & G & $\begin{array}{l}\text { Guest } \\
\text { Researcher }\end{array}$ & B. Miller & Semantics of mathematical documents \\
\hline $\begin{array}{l}\text { Adam } \\
\text { Meier }\end{array}$ & $\begin{array}{l}\text { University } \\
\text { of Colorado }\end{array}$ & G & PREP & E. Knill & Quantum computer simulation \\
\hline $\begin{array}{l}\text { Michael } \\
\text { Mullan }\end{array}$ & $\begin{array}{l}\text { University } \\
\text { of Colorado }\end{array}$ & G & PREP & E. Knill & Quantum computing theory \\
\hline $\begin{array}{l}\text { Yanbao } \\
\text { Zhang }\end{array}$ & $\begin{array}{l}\text { University } \\
\text { of Colorado }\end{array}$ & G & PREP & E. Knill & Tests of local realism \\
\hline $\begin{array}{l}\text { Catherine } \\
\text { Baker }\end{array}$ & $\begin{array}{l}\text { De Pauw } \\
\text { University }\end{array}$ & $\mathrm{U}$ & SURF & B. Cloteaux & Graph toughness conjecture \\
\hline $\begin{array}{l}\text { Brian } \\
\text { Clanton }\end{array}$ & $\begin{array}{l}\text { Rochester } \\
\text { Instiute of } \\
\text { Technology }\end{array}$ & $\mathrm{U}$ & SURF & S. Ressler & $\begin{array}{l}\text { Declarative 3D and web based } \\
\text { graphics }\end{array}$ \\
\hline $\begin{array}{l}\text { Jeffrey } \\
\text { Jacobs }\end{array}$ & $\begin{array}{l}\text { University } \\
\text { of Maryland }\end{array}$ & $\mathrm{U}$ & SURF & A. Mink & LDPC error correction on GPUs \\
\hline $\begin{array}{l}\text { Andrew } \\
\text { Keller }\end{array}$ & $\begin{array}{l}\text { De Pauw } \\
\text { University }\end{array}$ & $\mathrm{U}$ & SURF & J. Terrill & Visualization in cybersecurity \\
\hline $\begin{array}{l}\text { Connor } \\
\text { MacKenzie }\end{array}$ & $\begin{array}{l}\text { Westminster } \\
\text { College }\end{array}$ & $\mathrm{U}$ & SURF & H. Cohl & $\begin{array}{l}\text { Associated Legendre function repre- } \\
\text { sentations }\end{array}$ \\
\hline $\begin{array}{l}\text { Divya } \\
\text { Mouli }\end{array}$ & $\begin{array}{l}\text { Carnegie } \\
\text { Mellon }\end{array}$ & $\mathrm{U}$ & SURF & J. Terrill & $\begin{array}{l}\text { Gesture in an immersive visualization } \\
\text { environment }\end{array}$ \\
\hline $\begin{array}{l}\text { Johnathan } \\
\text { Orthwein }\end{array}$ & Columbia & $\mathrm{U}$ & SURF & G. McFadden & $\begin{array}{l}\text { Spectral analysis for the detection of } \\
\text { gases }\end{array}$ \\
\hline $\begin{array}{l}\text { Katelyn } \\
\text { Weber }\end{array}$ & $\begin{array}{l}\text { Montana } \\
\text { State }\end{array}$ & $\mathrm{U}$ & SURF & S. Glancy & Quantum state estimation \\
\hline $\begin{array}{l}\text { Jason } \\
\mathrm{Wu}\end{array}$ & Berkeley & $\mathrm{U}$ & SURF & F. Hunt & $\begin{array}{l}\text { Stochastic models of gene regulatory } \\
\text { networks }\end{array}$ \\
\hline $\begin{array}{l}\text { Joel Mus- } \\
\text { kin-Pierret }\end{array}$ & $\begin{array}{l}\text { Washington } \\
\text { University }\end{array}$ & $\mathrm{U}$ & STEP & J. Terrill & Visualization \\
\hline $\begin{array}{l}\text { Michael } \\
\text { Ekstrom }\end{array}$ & $\begin{array}{l}\text { Montgomery } \\
\text { Blair HS }\end{array}$ & HS & SHIP & J. Terrill & Visualization for cybersecurity \\
\hline $\begin{array}{l}\text { Jonathan } \\
\text { Ibers }\end{array}$ & $\begin{array}{l}\text { Poolesville } \\
\text { HS }\end{array}$ & HS & SHIP & J. Terrill & Visualization for cybersecurity \\
\hline
\end{tabular}

Legend G: Graduate student

U: Undergraduate student

HS: High school student

PREP: NIST Professional Research Experience Program (Boulder)

SHIP: Summer High-school Internship Program

STEP: Student Employment Program

SURF: NIST Summer Undergraduate Student Fellowship Program 


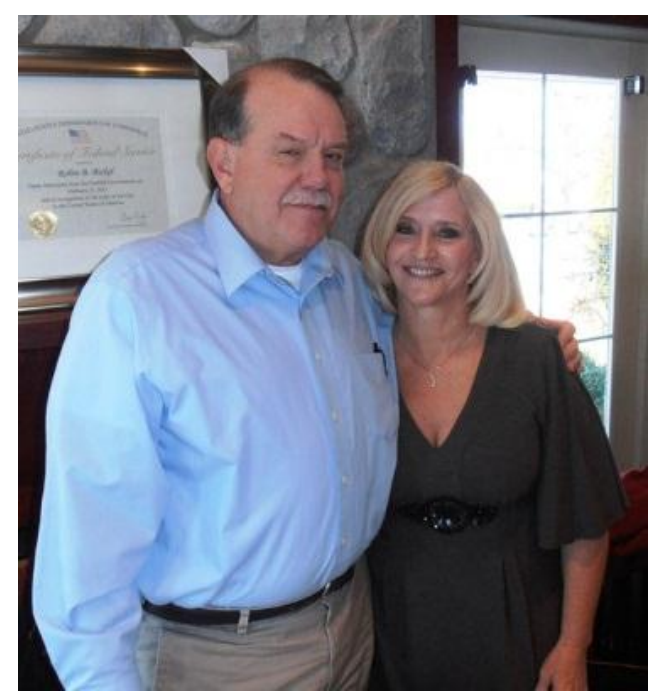

Figure 2. Robin Bickel and husband Keith. Robin retired in February 2012 after serving as Division Secretary for 23 years.
Robin Bickel, ACMD's Division Secretary, retired from NIST in February 2012 after 36 years of Federal service. Robin began her assignment as Division Secretary in 1989. An exemplary secretary, Robin received the ITL Outstanding Support Award on two occasions.

John Kelso, a member of ACMD's High Performance Computing and Communication Group for nine years, left the Federal service in September 2012. John was a key member of the team developing the software infrastructure for HPCV's immersive visualization facility. He resides in Blacksburg, VA.

Agnes (Abbie) O'Gallagher of ACMD-Boulder retired in November 2012 after 27 years of service. During her career at NIST Abbie provided support for ACMD efforts in cryocooler modeling, DNA sequence alignment, and CT scanner modeling. She was also an active member of ITL's Diversity Committee, where she organized the very successful ITL Diversity Book Club.

Peter Ketcham resigned from NIST in October 2011 after service as a mathematician at NIST for 14 years. During the late 1990s Ketcham worked with David Feder of the NIST Physics Lab and others to develop visualizations of models of the newly discovered Bose Einstein condensate. These striking images made their way to the covers of Physics Today, Optics and Photonics News, Parity, and the lead illustration in a Scientific American article. In 2000 Ketcham was a joint recipient of the NIST Bronze Medal.

Lijun Ma, an ITL guest researcher for seven years, left NIST in October 2011 to take a position as a research assistant professor in the Biomedical Engineering Department at Washington University in St. Louis. An expert in quantum optics, Ma was a significant contributor to ITL's experimental efforts in quantum communications. An incredibly productive researcher, Ma co-authored 52 papers in journals and conference proceedings during his six years at NIST. Ma received his PhD in Physics from Tsinghua University in China in 2001. He recently became a permanent resident of the US.

\section{Students}

During FY 2012 ACMD was able to support the work of 20 student interns, including nine in the Student Undergraduate Research Fellowship (SURF) program, three graduate students in Boulder's Professional Research Experience Program (PREP), and two high school volunteers. We also hosted three graduate students from French universities; this was facilitated by a Memorandum of Understanding signed in late 2009 between ITL and the Institut Supérieur d'Informatique, de Modélisation et de leurs Applications (ISIMA) at the University of Clermont-Ferrand. See Table 2 for a complete listing.

This year ACMD issued a grant to the National Physical Sciences Consortium (NPSC) to support the graduate research of three students. The NPSC is a partnership between government agencies and laboratories, industry, and higher education, whose goal is to increase the number of American citizens with graduate degrees in the physical sciences and related engineering fields, emphasizing recruitment of a diverse applicant pool ${ }^{10}$. As part of their studies, NPSC students spend at least one summer in residence at NIST. The students selected for NIST support beginning in the fall of 2012 are:

- Earl Bellinger, Indiana University: High performance computing (J. Terrill, mentor)

- Bryan Gard, Louisiana State University, Quantum information (M. Knill, mentor)

- Franz Sauer, University of California at Davis, Scientific visualization (J. Terrill, mentor)

${ }^{10}$ http://www.npsc.org/ 


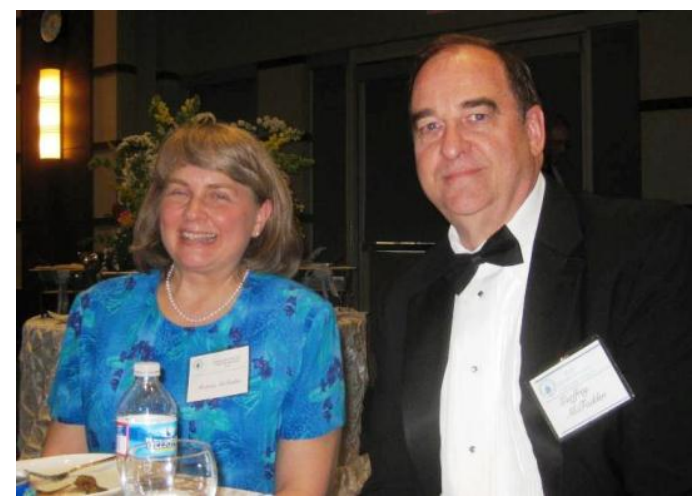

Figure 4. Geoffrey McFadden and spouse, Roberta, at the Washington Academy of Sciences Awards Ceremony.

\section{Recognition}

Division staff garnered a number of professional recognitions during the past year. These are described below.

Geoffrey McFadden of ACMD was named a Fellow of the Society for Industrial and Applied Mathematics (SIAM) at the society's annual meeting on July 10, 2012 in Minneapolis. McFadden was cited for advances in mathematics applied to fluid dynamics, solidification, and the interaction of the two, using sharp and diffuse interface theories. SIAM inaugurated its Fellows Program in 2008 to recognize members who have made outstanding contributions to industrial and applied mathematics.

Geoffrey McFadden was also selected to receive the 2012 Washington Academy of Sciences (WAS) Award for merit and distinction in Mathematics and Computer Science. McFadden is one of 10 awardees representing multiple fields in the sciences and engineering who was recognized at the WAS annual meeting and awards banquet held on May 10 in Arlington, VA. Previous winners of the WAS award in mathematics and computer science include James Yorke, Eitan Tadmor, and Victor Basili of the University of Maryland, and Stuart Katzke of ITL. An applied mathematician, McFadden's contributions have been in mathematical modeling in fluid dynamics, solidification, and pattern formation, with application to materials science and other fields.

On September 28, 2012 Ronald Boisvert of ACMD received an Outstanding Alumni distinction from the Purdue University College of Science. Purdue's Computer Science Department, which celebrates its $50^{\text {th }}$ anniversary this year, developed the nomination. Boisvert received a Ph.D. in computer science from Purdue in 1979.

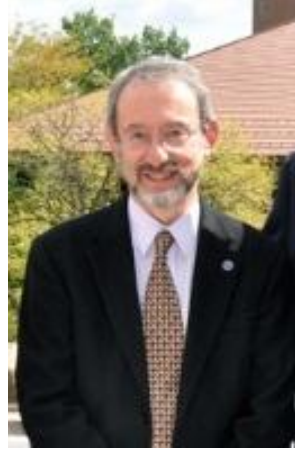

Figure 3. Ronald Boisvert was named an Outstanding Alumni by the Computer Science Department at Purdue. 


\section{Part II}

\section{Features}

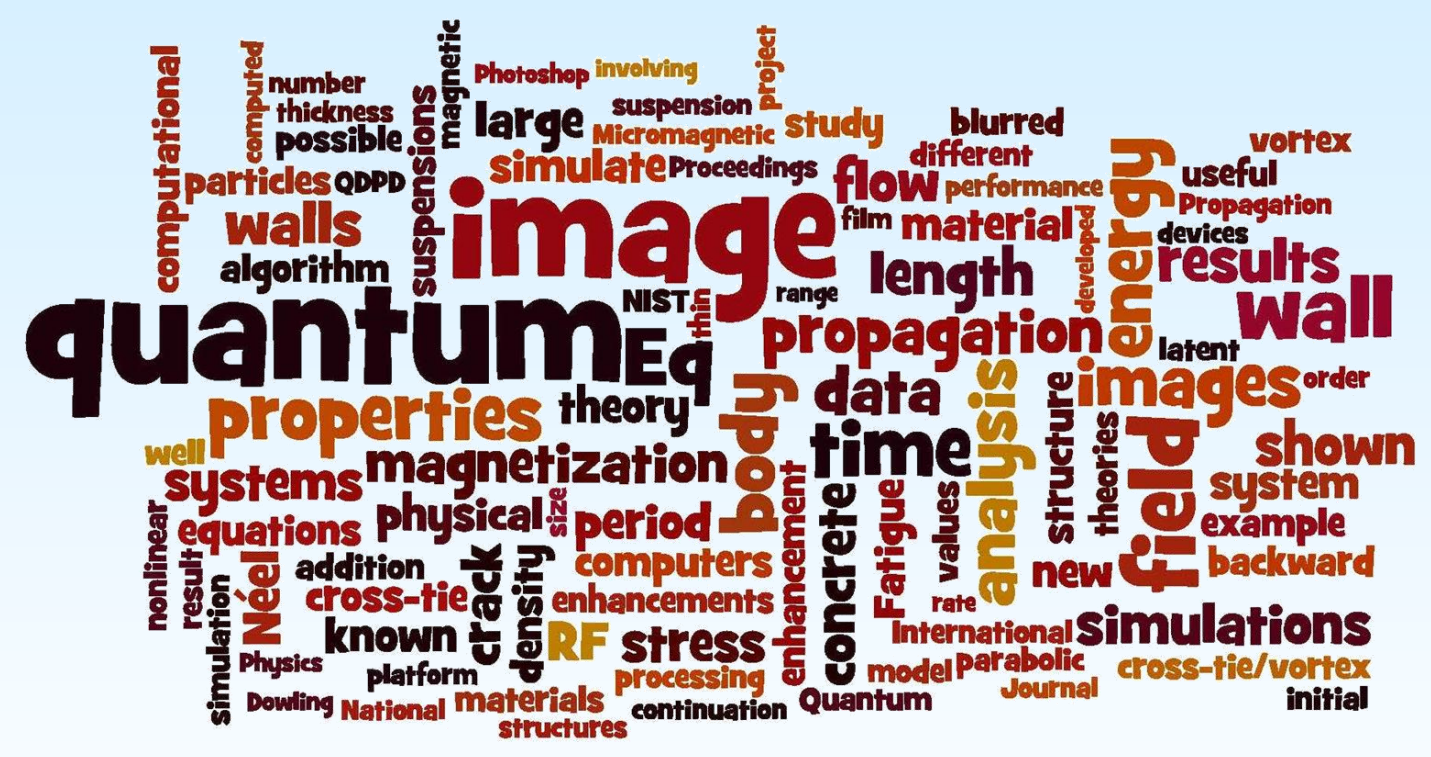




\section{Quantum Algorithms for Quantum Field Theories}

\begin{abstract}
Quantum field theories are needed to describe systems in which the effects of quantum mechanics and special relativity are both important. The Standard Model of particle physics, which aims to explain all physical phenomena excluding gravity, is a quantum field theory. Calculating quantitative predictions from a quantum field theory can be extremely difficult. In particular, no efficient algorithm is known for classical computers to simulate the dynamics of quantum field theories involving strongly-interacting particles. In this project, we are developing methods by which quantum computers could efficiently simulate the dynamics of quantum field theories, even when the interactions between particles are strong. If quantum computers are successfully built, our methods could be of practical use in predicting the outcomes of experiments at particle accelerators. Furthermore, by studying whether quantum computers can efficiently simulate the Standard Model, we hope to address the fundamental computational power of the universe by either confirming or refuting the oft-repeated claim that the universe is equivalent in computational power to a quantum computer.
\end{abstract}

\section{Stephen Jordan}

Ever since Richard Feynman's original proposal for quantum computers, one of the primary applications envisioned has been efficient simulation of other quantum systems. In fact, it has been conjectured that quantum computers are universal simulators, which can simulate all physical systems using computational resources that scale polynomially with the system's number of degrees of freedom. Quantum field theories have posed a challenge in that the set of degrees of freedom is formally infinite. In this project, we are working to show that quantum computers, if built, could nevertheless efficiently simulate quantum field theories at bounded energy scales.

So far, we have devised a quantum algorithm to simulate the simplest nontrivial quantum field theory we could find, namely massive phi-fourth $\left(\varphi^{4}\right)$ theory [1]. Our algorithm calculates scattering probabilities in relativistic particle collisions similar to those that occur in particle accelerators such as the Large Hadron Collider. Our algorithm runs in time scaling polynomially in the number of particles, their energy, and the desired precision, and works even when the coupling between particles is strong. In contrast, known polynomial-time classical algorithms break down when computing dynamical quantities such as scattering amplitudes in situations involving strong coupling or requiring high precision.

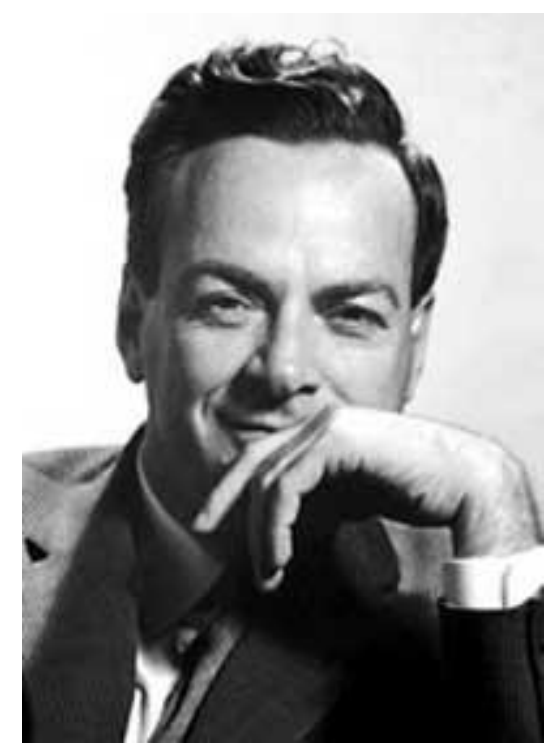

Figure 5. Richard Feynman proposed the idea of a universal quantum simulator in 1982. He postulated that while a classical Turing machine would require exponential resources to simulate quantum phenomena, a simulator based itself on quantum principles would not.

Photo: http://en.wikipedia.org/wiki/File:Richard_Feynman_Nobel.jpg

Although we believe the algorithm of [1] represents a major first step in simulating quantum field theories, much work remains to be done. In particular, matter consists of two main classes of particles called bosons and fermions. $\varphi^{4}$ theory involves only bosons. Currently, we are working to generalize our algorithm to treat quantum field theories involving fermions [2]. In future work we will attempt to extend our algorithm to handle massless particles and gauge symmetries. If successful, this will result in a quantum algorithm capable of efficiently simulating the Standard Model of particle physics, which encompasses all known physical processes not involving gravity.

In the theory of computational complexity, it has proven fruitful to categorize computational problems into those that can be solved using a number of computational steps that scales polynomially with the input size, and those that require a larger-than-polynomial (typically exponential) amount of computation. Being coarse-grained, this distinction is highly independent of the details of the model of computation used to define the notion of computational steps. In fact, prior to the discovery of quantum computation, it was widely believed that the polynomial vs. superpolynomial categorization was completely robust; no realistic form of computation was believed to be more powerful than standard Turing machines. This assertion can be formalized as the complexity-theoretic Church-Turing thesis: anything that can be computed in polynomial 
time can be computed in polynomial time on a Turing machine.

The philosophical status of the complexitytheoretic Church-Turing thesis has long been a source of discomfort. Being unwilling to call it either a theorem or a definition, mathematicians deferred the issue of identifying the status of the claim by calling it a "thesis". In the 1980s, David Deutsch put forth the interpretation that the complexity-theoretic ChurchTuring thesis only becomes meaningful when interpreted as a claim about physics: "Anything that can be computed in polynomial-time by physical means can be computed in polynomial-time by a Turing machine." Furthermore, building on previous observations by Feynman, Deutsch conjectured that this claim is false, due to quantum-mechanical processes that seem to be exponentially hard to simulate using standard classical Turing machines.

The point of view espoused by Feynman and Deutsch gained much support from Peter Shor's spectacular 1994 discovery of a polynomial-time quantum algorithm for factoring $n$-digit integers, a problem widely believed to require superpolynomial time to solve classically. The complexity-theoretic ChurchTuring thesis was soon superseded by the quantum Church-Turing hypothesis: "Anything that can be computed in polynomial-time by physical means can be computed in polynomial time by a quantum Turing machine." Paralleling the history of classical computation, many theoretical models of quantum computation were proposed, such as quantum generalizations of logic circuits, and quantum analogues of simulated annealing, and all of these were proven to be simulatable with polynomial overhead by quantum Turing machines. Furthermore, polynomial-time quantum algorithms were eventually developed to simulate various quantum phenomena in nature, such as chemical reactions, and the dynamics of spin systems. All of these simulation results gave additional support to the validity of the quantum Church-Turing thesis, and the notion that the universe is equivalent to a large quantum computer began to permeate the popular press [3].

Although potentially very valuable if quantum computers are successfully built, the polynomial-time quantum algorithms for simulating chemical reactions and spin dynamics do not provide fully convincing evidence for the validity of the quantum Church-Turing thesis. Fundamentally, all of these quantum algorithms for simulating were designed to simulate many-body systems, and have runtime scaling polynomially in the number of degrees of freedom being simulated. In principle, a field has infinitely many degrees of freedom, as it is specified by its value at every point in space. In contrast, a system of $n$ particles has only $3 n$ degrees of freedom: the three spatial coordinates of each particle. Direct application of existing algorithms is not possible and one is faced with an open question: can quantum computers in polynomial time simulate the dynamics of quantum field theories such as the standard model?

The obvious response to the infinite degrees of freedom in a field is to discretize. This is invariably done in simulating classical fields, for instance by finite-element methods. What we have learned in the course of our research so far is that discretizing space on a lattice yields an efficient method for simulating quantum field theories on quantum computers and the discretization errors converge to zero as the lattice spacing is taken to zero, just as one would hope. However, the means by which this convergence is achieved has some uniquely quantum-mechanical quirks. Specifically, the parameters of the physical model, such as the particle mass and coupling strength, must be adjusted as functions of the lattice spacing in order to achieve convergence to a continuum limit. This infamous aspect of quantum field theory is known as renormalization, and its conceptual and quantitative elucidation was only achieved in the 1970s and 1980s, led by Kenneth Wilson.

The main technical aspects of our algorithm for simulating $\varphi^{4}$ theory are the efficient preparation of physically realistic initial states, and the renormalization-based analysis of discretization errors. Both of these aspects are highly dependent on the underlying physics. Thus, extension of our results to more general quantum field theories, such as the Standard Model remains nontrivial. Our suspicion is that quantum computers can in polynomial time simulate the entire Standard Model. Proving this remains a guiding goal for our future work. On the other hand, if this turns out to be false, we can instead look forward to quantum field computers, at least as a theoretical construct, that are exponentially more powerful than standard quantum computers.

\section{References}

[1] S. Jordan, K. S. M. Lee, and J. Preskill, Quantum Algorithms for Quantum Field Theories, Science 336 (2012) 1130-1133.

[2] S. Jordan, K. S. M. Lee, and J. Preskill, Quantum Algorithms for Fermionic Quantum Field Theories, in preparation.

[3] S. Lloyd, Programming the Universe: A Quantum Computer Scientist Takes on the Cosmos, Vintage Press, 2007.

\section{Participants}

Stephen Jordan (ACMD), Keith Lee (University of Pittsburgh), John Preskill (Caltech) 


\section{Micromagnetic Investigation of Periodic Cross-Tie/Vortex Wall Geometry}

A careful examination of magnetization structure in thin film elements has been conducted via a systematic series of micromagnetic simulations. Depending on the element dimensions, a particular structure known as a cross-tie wall can occur in these elements. The physical processes supporting cross-tie wall formation are revealed and a phase diagram that predicts the existence or not of cross-tie walls is produced. These results are of interest because they differ from analytic results in the infinitely thin film limit. The results are of practical interest because the performance of devices such as computer hard drive read heads and nascent magnetic memory technology such as MRAM are dependent on fine details of magnetization structure in thin film elements.

\section{Michael J. Donahue}

Magnetic materials are generally classified into two categories, "hard" materials that hold a remnant magnetization and resist change to the magnetization, and "soft" materials with magnetization that is easily changed by an applied magnetic field. The former are used, for example, in permanent magnets and computer hard drive platters, while the latter are in transformers, magnetic field sensors, computer hard drive read heads, and in new classes of magnetic memory devices such as MRAM.

As a result of energy minimization, the magnetization in magnetic materials tends to form domains of more or less uniformly magnetized regions separated by narrow transition regions called domain walls, as shown in Figure 6. The number and type of domains and domain walls can influence the behavior and response speeds of magnetic devices.

In thin magnetic films of the type primarily deployed in present day devices, the archetypal domain walls are Néel walls, where the magnetization in the wall rotates in the plane of the film, and Bloch walls, where the magnetization rotation is about the axis normal to the plane of the wall. Néel walls occur in very thin films, Bloch walls are found in thicker samples, and numerous transitional structures [1-3] are obtained at intermediate thicknesses. One of the more interesting transitional structures is the cross-tie/vortex (or simply cross-tie) wall which alternates cross-ties and vortices between counter-rotating segments of Néel walls. The configuration in Figure 6a shows one crosstie with an " $X$ "-shaped magnetization pattern in the center of the strip, and vortices on either side with circular magnetization patterns with counterclockwise
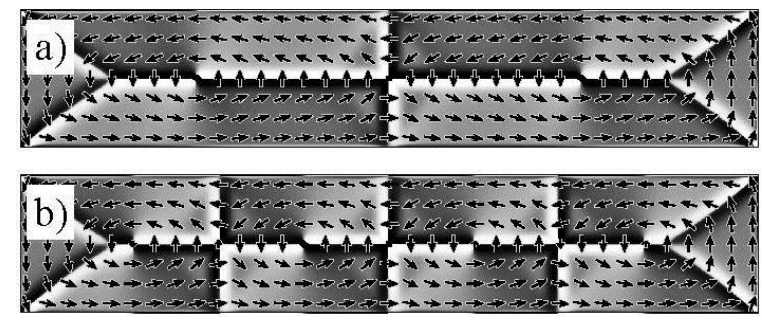

Figure 6. Two remanent equilibrium states for identical $2645 \mathrm{~nm}$ $\times 530 \mathrm{~nm} \times 32 \mathrm{~nm}$ NiFe film rectangles. Grayscale indicates magnetic charge, $-\nabla \cdot \mathbf{M}$. Each of these configurations is energetically stable, however configuration (b) has lower energy.
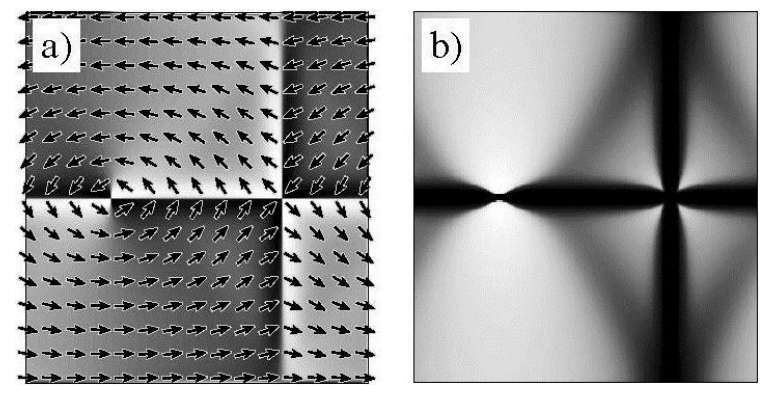

Figure 7. Detail of equilibrium configuration for strip with width $Y=200 \ell_{e x}$, thickness $Z=2 l_{\mathrm{ex}}$, and X-period length $317 \mathrm{lex}_{\mathrm{ex}}$ which is the natural period length for this $Y$ and $Z$ ): (a) Magnetization pattern, (b) stray-field energy density. In (a) the grayscale indicates magnetic charge; in (b) the grayscale runs from white (low energy density) to black (high energy density).

flow about each vortex core. The connecting region between the cross-tie and each vortex has the form of a $180^{\circ}$ Néel wall. Cross-tie/vortex walls are observed experimentally [4-6], in micromagnetic simulations [69], and in theory $[10,11]$. In finite samples, this wall type appears in low remanence closed-flux Landau patterns; if the structure is long enough, then multiple cross-tie/vortex pairs may appear (Figure 6b).

In the following, energy densities are reported relative to the material magnetostatic energy density $K_{\mathrm{d}}$ $=\mu_{0} M_{\mathrm{s}}^{2} / 2$, and lengths relative to the magnetostaticexchange length $\ell_{\mathrm{ex}}=\left(A / K_{\mathrm{d}}\right)^{1 / 2}$. $\left(M_{\mathrm{s}}\right.$ is the saturation magnetization in $A / \mathrm{m}^{2}$, and $A$ is the material exchange coefficient in $\mathrm{J} / \mathrm{m}$ ). This makes the results applicable to any soft magnetic film. For the NiFe material in Figure $6, K_{\mathrm{d}}=400 \mathrm{~kJ} / \mathrm{m}$ and $\ell_{\mathrm{ex}}=5 \mathrm{~nm}$. All results in this report are based on simulations using the OOMMF micromagnetic program [12].

To understand how cross-tie/vortex structures lower the energy density as compared to a Néel wall, examine the checkerboard pattern formed by the magnetic charges in Figure 6 or the close-up shown in 


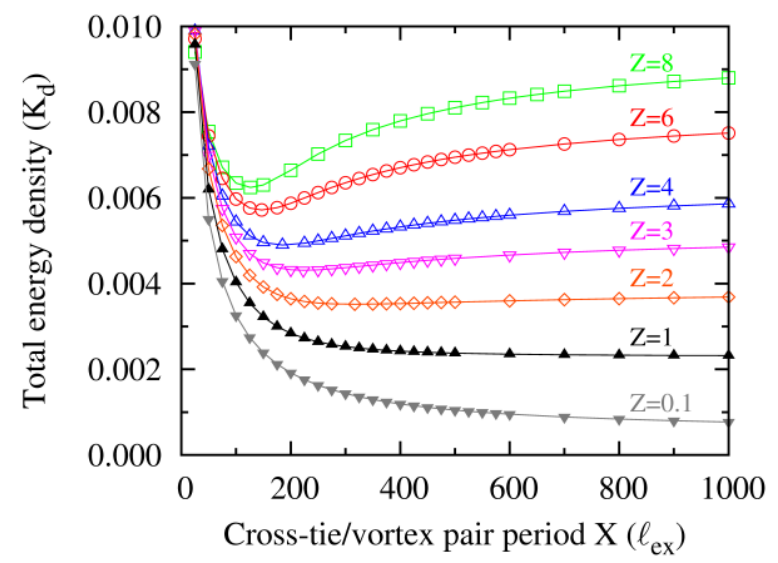

Figure 8. Average total energy density as a function of period length $X$ for simulations with width $Y=200 \ell_{\mathrm{ex}}$ for various thicknesses $Z$ (measured in $\ell_{\mathrm{ex}}$ ). Symbols represent simulation results, lines are a guide to the eye. Each of the curves with $Z \geq 2 \ell_{\mathrm{ex}}$ exhibits a minimum value between $125 \ell_{\mathrm{ex}}$ and $320 \ell_{\mathrm{ex}}$. The curves for $Z \leq 1 \ell_{\mathrm{ex}}$ are monotonically decreasing with $X$ across the displayed range.

Figure 7a. Looking first at the section of the Néel portion of the wall between the left hand vortex and the cross-tie, and moving from bottom to top across the wall, the magnetization rotates counterclockwise. This configuration produces negative magnetic charge on the lower half of the strip (dark region) and positive charges on the upper half (light region). This sets up a stray field running from the positive charges to the negative charges, counter to the magnetization in the center of the wall, making the wall center a region of high stray field energy density. However, in the checkerboard pattern, stray field between charge blocks runs not only up and down across the Néel sections of the wall, but also left and right horizontally parallel to the wall. The orientation of the magnetization about each vortex core is such that it aligns with the stray field from the nearby charge blocks, so that the regions above and below each vortex are regions of low stray field energy density. This effect is displayed directly in Figure $7 b$, where the light-colored low energy density regions above and below the vortex core are clearly visible. Conversely, the areas around the cross-tie are dark-colored high energy density regions. More detailed analysis of these simulations confirms that at these thicknesses the vortex/cross-tie wall is energetically favorable relative to a Néel wall because of the magnetostatic energy savings around the vortex. This result stands in contrast to analytic studies in the infinitely thin film limit $[10,11]$, which find the cross-tie to be energetically favored and the vortex to be disfavored.

Vortices and cross-ties pin easily on material defects, affecting wall movement and magnetization evolution. Also the cross-tie/vortex wall structure is periodic, and the energy density in the wall is affected by the period length. Therefore it is useful for design purposes, as well as being a matter of theoretical interest, to understand the mechanisms behind the formation of cross-tie walls and to be able to predict the occurrence and cross-tie/vortex period length in this type of wall. In order to study this structure free from effects of edge domains in finite-length samples, a systematic series of simulations was performed with periodic boundary conditions along the direction of the wall. (This requires some care because the field from each periodic image affects the magnetization in all the other periodic images [13].) In this study the strip width and thickness is fixed, a period length is selected and then the magnetization is relaxed until a (local) energy minimum is reached. The energy density for this equilibrium is recorded, and the process is repeated for a different period length.

In Figure 8, each point marks the energy density at equilibrium resulting from a simulation with width $Y$ $=200 \ell_{\mathrm{ex}}$ at the indicated thickness $Z$ and period length $X$. There is one cross-tie/vortex pair in each period, so as the period length $X$ grows large the wall becomes essentially two Néel segments interrupted by a cross-tie and vortex at either end. This is evident in the behavior of the curves for large $X$, as for each thickness $Z$ the curve asymptotically converges to the energy density of the associated Néel wall. For small period lengths $\left(X<100 \ell_{\mathrm{ex}}\right)$ the energy density grows sharply as the exchange energy resists compression of the complex cross-tie/vortex structure.

In the thicker films there is a unique minimum on each curve, which corresponds to a "natural" period length - this is the period length that minimizes the energy for a cross-tie/vortex wall at the given film thickness and strip width. Note though that each point in Figure 8 is a stable equilibrium configuration under the fixed period assumption. So if the period length is constrained by geometry or other means such as pinning defects, then period lengths other than the natural length are possible, as seen in Figure 6.

For thinner films $\left(Z\right.$ under about $1 \ell_{\mathrm{ex}}$ for $Y=200$ $\ell_{\mathrm{ex}}$ ), the energy density curves are monotonically decreasing. This means that in a thin, infinite strip, if the cross-tie and vortex are not pinned by some means, they will spread out indefinitely leaving behind a plain Néel wall. This is consistent with the experimental result that cross-tie/vortex walls are not observed in ultra-thin films. One can obtain a good estimate of the critical thickness below which pure Néel walls are favored by finding the natural period length for each thickness and fitting a curve through the data. This curve has a pole at the critical thickness. The pole/critical thickness is a function of the strip width; the data from simulation series at various strip widths is collected on the log-log plot in Figure 9. Across this range of values the data are fit quite well by the rela- 
tion $Z_{\text {crit }} \approx 30 / Y^{2 / 3}$. This graph represents a phase diagram for walls in thin film strips-strips with thickness/width combinations below this line will prefer Néel walls, while thickness/width combinations above this line will favor cross-tie/vortex walls. Additional details and results are available in [14].

\section{References}

[1] R. Kirchner and W. Döring, Thin Films: Domain Walls and Related Topics, Journal of Applied Physics 39 (1968), 855-856.

[2] D. V. Berkov, K. Ramstöck, and A. Hubert, Solving Micromagnetic Problems. Towards an Optimal Numerical Method, Physicus Status Solidi (a) 137 (1993), 207225.

[3] K. Ramstöck, W. Hartung, and A. Hubert, The Phase Diagram of Domain Walls in Narrow Magnetic Strips, Physicus Status Solidi (a) 155 (1996), 505-518.

[4] R. Ploessl, J. N. Chapman, A. M. Thompson, J. Zweck, and $\mathrm{H}$. Hoffmann, Investigation of the Micromagnetic Structure of Cross-tie Walls in Permalloy, Journal of Applied Physics 73 (1993), 2447-2452.

[5] A. Hubert and R. Schäfer, Magnetic Domains. Springer, 1998.

[6] S. Huo, G. Pan, D. J. Mapps, W. W. Clegg, G. Heydon, W. M. Rainforth, H. A. Davies, J. E. L. Bishop, J. W. Tucker, and M. R. J. Gibbs, Magnetic Force Microscopy and Micromagnetic Study of Cross-tie Wall Structures in $\mathrm{Co}_{91} \mathrm{Nb}_{6} \mathrm{Zr}_{3}$ Amorphous Thin Films, Journal of Applied Physics 87 (2000), 1096-1102.

[7] M. Redjdal, A. Kakay, M. F. Ruane, and F. B. Humphrey, Cross-tie Walls in Thin Permalloy Films, IEEE Transactions on Magnetics 38 (2002), 2471-2473.

[8] W. Rave and A. Hubert, Magnetic Ground State of a Thin-Film Element, IEEE Transactions on Magnetics $\mathbf{3 6}$ (2000), 3886-3899,

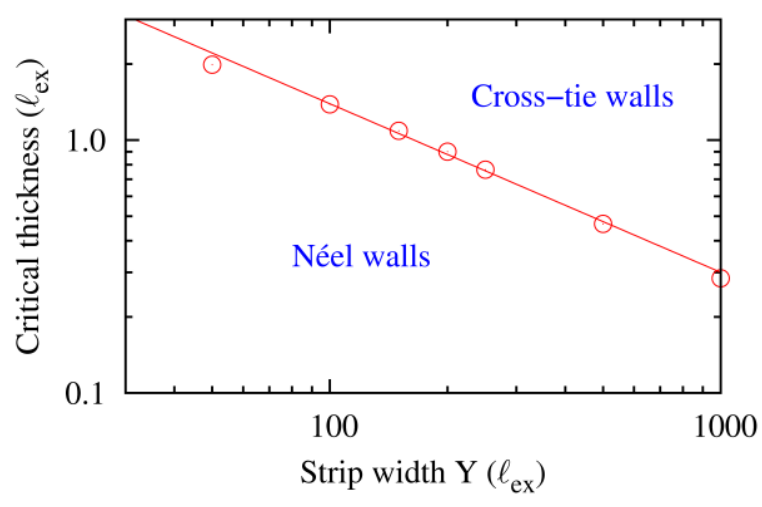

Figure 9. Critical strip thickness for which the pure Néel wall has lower energy density than a cross-tie/vortex wall of any period, as a function of strip width $Y$. Data points represent results from simulations; the line is the fit curve $30 / Y^{2 / 3}$.

[9] R. Hertel, Thickness Dependence of Magnetization Structures in Thin Permalloy Rectangles, Zeitshrift für Metallkunde 93 (2002), 957-962.

[10] F. Alouges, T. Rivière, and S. Serfaty, Néel and Crosstie Wall Energies for Planar Micromagnetic Configurations, ESAIM: Control, Optimization and Calculus of Variations 8 (2002), 31-68.

[11] A. DeSimone, H. Knüpfer, F. Otto, 2-d Stability of the Néel Wall, Calculus of Variations and Partial Differential Equations 27 (2006), 233-253.

[12] M. J. Donahue and D. G. Porter, OOMMF Object Oriented Micromagnetic Framework, National Institute of Standards and Technology, Gaithersburg, MD. http://math.nist.gov/oommf.

[13] K. M. Lebecki, M. J. Donahue, and M. W. Gutowski, Periodic Boundary Conditions for Demagnetization Interactions in Micromagnetic Simulations, Journal of Physics D - Applied Physics 41 (2008), 175005.

[14] M. J. Donahue, Micromagnetic Investigation of Periodic Cross-Tie/Vortex Wall Geometry, Advances in Condensed Matter Physics (2012), 908692 (8 pages). 


\section{Continuation Backward in Time in 2D Nonlinear Parabolic Equations, and an Experiment in Deblurring Nonlinearly Blurred Imagery}

Identifying sources of ground water pollution by solving the advection dispersion equation (ADE) backward in time, the so-called hydrologic inversion problem, is of major interest in environmental engineering [1]. In an entirely different direction, ACMD has pioneered the use of time-reversed fractional and logarithmic diffusion equations in blind image deblurring in such areas as MRI and PET brain scans, nanoscale scanning electron and Helium ion micrographs, and Hubble space telescope imagery [2]. Here, the recorded blurred image is viewed as the solution at time $t=1$ in an appropriate linear parabolic equation, and the desired ideal image is the corresponding initial value at time $t=0$. These and other successful inverse reconstructions have generated a great deal of current mathematical interest in backward in time continuation in parabolic equations. Not surprisingly, very little is known either analytically or computationally in the significantly more challenging area of backward continuation in nonlinear multidimensional parabolic equations. We discuss instructive examples illustrating the unexpected influence of certain types of nonlinearities. Visually and statistically indistinguishable blurred images are presented, with vastly different deblurring results. These examples indicate that how an image is nonlinearly blurred is critical, in addition to the amount of blur. The equations studied represent nonlinear generalizations of Brownian motion, and the blurred images may be interpreted as visually expressing the results of novel stochastic processes.

\section{Alfred S. Carasso}

It is instructive to study 2D second order parabolic equations such as the following,

$$
\begin{aligned}
& w_{t}=\gamma r(w) \nabla \cdot\{q(x, y, t) \nabla w\} \\
& \quad+c \sqrt{w} w_{x}+d\left(\cos ^{2} w\right) w_{y} \quad \Omega \times(0, T) \\
& w(x, y, 0)=g(x, y)
\end{aligned}
$$

on the unit square $\Omega$, with homogeneous Neumann conditions on the boundary of $\Omega$. Here $\gamma, c, d$ are appropriately chosen positive constants, $q(x, y, t)$ is a positive non-isotropic diffusivity, and the positive nonlinear function $r(w)$ is exponential in $w$. By varying the constants in $r(w)$ and in the nonlinearities in the $w_{x}$ and $w_{y}$ terms, a variety of interesting examples can be created. Numerous other types of nonlinearities can also
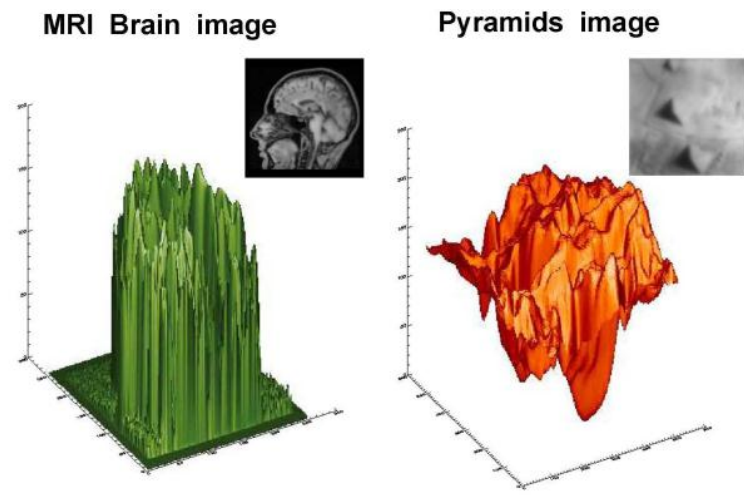

Figure 10. Images provide unusually interesting non negative initial values $g(x, y)$ that can be used to generate approximate values $f(x, y)$, for the exact solution $w(x, y, T)$ at time T in Eq. (1).

be considered. Such nonlinear equations may occur in diverse scientific and engineering contexts.

Useful computational exploration of ill-posed backward continuation in Eq. (1) requires intelligent choices for the initial values $g(x, y)$, which must be sufficiently complex and rich in high frequency components in order to adequately probe behavior. As shown in Figure 10, images can provide unusually interesting candidates $g(x, y)$. Irrespective of the ultimate intended application of Eq. (1), an efficient exploration strategy can be based on creating fictitious blurred image data by using sharp images for $g(x, y)$, and selecting the corresponding solution at time $T>0$ as the given continuation data. Successful backward continuation from $t=T$ to $t=0$ would recover the sharp image. An important advantage is that visual recognition can provide useful and immediate evaluation of the degree of success or failure in that continuation. This can then be translated into the intended application of Eq. (1), unrelated to imaging.

Hazardous backward continuation. For any initial value $g(x, y)$, Eq. (1) has a unique solution $w(x, y, T)$ at time $T>0$. The function $w(x, y, T)$ belongs to a very restricted class of smooth functions and is seldom known exactly. Rather, only approximate values $f(x, y)$ are typically known. A major challenge is to obtain a useful approximation to the true initial value $g(x, y)$, by backward continuation from $t=T$ using the noisy data $f(x, y)$. Indeed, if $\|w(., T)-f\|_{2} \leq \delta$ with small $\delta>0$, and we constrain the continuations to satisfy a prescribed $L^{2}$ bound $M$ at $t=0$, the difference between any two possible continuations $w_{i}(x, y, t), i=1,2$, obeys the following stability inequality 


\section{NONLINEAR PARABOLIC BLURRING OF SHARP CARRIER IMAGE}

(G) Original sharp image

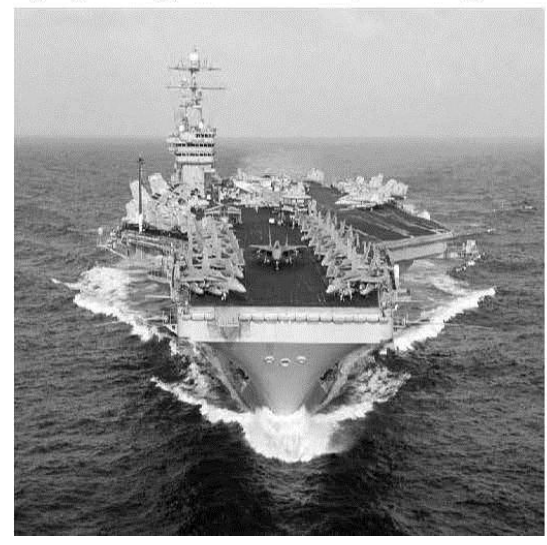

(H) Blur with $\mathrm{c}=2.5, \mathrm{~d}=\mathbf{0 . 3}$

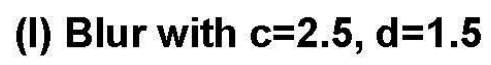

Figure 11. Nonlinear parabolic blurring of sharp USS Eisenhower image, by using it as the initial value g(x,y) in Eq. (1) with two different sets of constants $c, d$. Blurred images $(H)$ and $(I)$ are visually and statistically indistinguishable, with the same PSNR values, and almost identical $L^{1}, L^{2}$, and TV norm values.

$$
\left\|w_{1}(., t)-w_{2}(., t)\right\|_{2} \leq 2 M^{1-\mu(t)} \delta^{\mu(t)}, \quad 0 \leq t \leq T .
$$

In the best case scenario, that of a linear autonomous selfadjoint parabolic equation, we have $\mu(t)=t / T$ in Eq. (2), implying a systematic loss of reconstruction accuracy as $t \downarrow 0$. Nevertheless, as shown in [2], useful reconstruction may still be possible. However, in nonlinear equations deviating strongly from the autonomous selfadjoint case, $\mu(t)$ may exhibit rapid exponential decay as $t \downarrow 0$. The resulting loss of accuracy may then be so severe that useful reconstruction is not possible, despite the use of regularizing smoothness and non-negativity constraints. Examples of smooth, non-negative, physically plausible, yet false backward reconstructions are given in [3].

Van Cittert iterative procedure. Recently, a useful approach to such non-linear continuation problems as Eq. (1) has been discovered in ACMD. It is based on adapting an iterative procedure originating in the field of spectroscopy in the 1930's. This is the Van Cittert iteration [4], which was designed for solving 1D linear convolution integral equations $S g=f$, with explicitly known kernels having positive Fourier transforms. With suitable fixed $\lambda$ such that $0<\lambda<1$, and $h^{1}(x)=\lambda$ $f(x)$, Van Cittert's method solves $S g=f$ using the iteration

$$
h^{m+1}(x)=h^{m}(x)+\lambda\left\{f(x)-S\left\lfloor h^{m}(x)\right]\right\}, \quad m>1
$$

There is no explicitly known linear convolution operator $S$ in the case of Eq. (1). Instead, we can construct a discrete nonlinear solution operator $\Lambda$, which maps the initial value $g(x, y)$ into $w(x, y, T)$. We restrict attention to $256 \times 256$ pixel 8-bit images. Imposing a $256 \times 256$ mesh on the unit square, we use centered space differencing in Eq. (1), together with explicit time differencing, while lagging all non-linearities at the previous time step. With $T=N \Delta t$, the discrete solution operator $\Lambda$ is defined on $256 \times 256$ images $w^{0}$, by $\Lambda\left[w^{0}\right]=w^{N}$. Thus, $\Lambda$ applied to a given image uses that image as data $w^{0}$ at $t=0$ in the difference scheme, and marches that image $N$ time steps forward in time, to produce a blurred image $w^{N}$ at $T=N \Delta t$, in accordance with Eq. (1).

Given the sharp $256 \times 256$ image $\mathrm{g}(\mathrm{x}, \mathrm{y})$ as initial value in Eq. (1), applying the discrete solution operator $\Lambda$ to $g(x, y)$ produces a blurred image $f(x, y)$, which is a noisy approximation to the unknown, highly elusive, smooth, exact solution $w(x, y, T)$. Backward continuation from $t=T$ in Eq. (1) using the given data $f(x, y)$, requires solving the $2 \mathrm{D}$ nonlinear equation $\Lambda g=f$, in analogy with the simpler 1D linear equation $S g=f$ in Eq. (3). Accordingly, with suitable fixed $\lambda$ such that $0<$ $\lambda<1$, and $h^{1}(x, y)=\lambda f(x, y)$, Van Cittert nonlinear deblurring is defined by

(4) $h^{m+1}(x, y)=h^{m}(x, y)+\lambda\left\{f(x, y)-\Lambda\left[h^{m}(x, y)\right]\right\}, \quad m>1$

Nonlinear deblurring experiment. It is remarkable that Eq. (4) can be used successfully in backward continuation in a wide class of 2D nonlinear parabolic equations similar to Eq. (1). However, as indicated by Eq. (2), stability difficulties should be anticipated in parabolic equations deviating too strongly from the

Table 3. Behavior using Eq. (1) in nonlinear blurring in Figure 11 .

\begin{tabular}{|l|l|l|l|l|l|}
\hline $\begin{array}{l}\text { Image } \\
f(x, y)\end{array}$ & $\begin{array}{l}\text { Parameters } \\
c / d\end{array}$ & $\|f\|_{1}$ & $\|f\|_{2}$ & $\|\nabla f\|_{1}$ & $P S N R$ \\
\hline $\mathrm{G}$ & Not blurred & 139 & 153 & 4760 & $\infty$ \\
\hline $\mathrm{H}$ & $2.5 / 0.3$ & 134 & 147 & 1720 & 20 \\
\hline $\mathrm{I}$ & $2.5 / 1.5$ & 134 & 148 & 1770 & 20 \\
\hline
\end{tabular}




\section{NONLINEAR DEBLURRING OF CARRIER IMAGE}

(H) Blur with $\mathrm{c}=2.5, \mathrm{~d}=\mathbf{0 . 3} \quad$ After 100 VanCittert iterns.

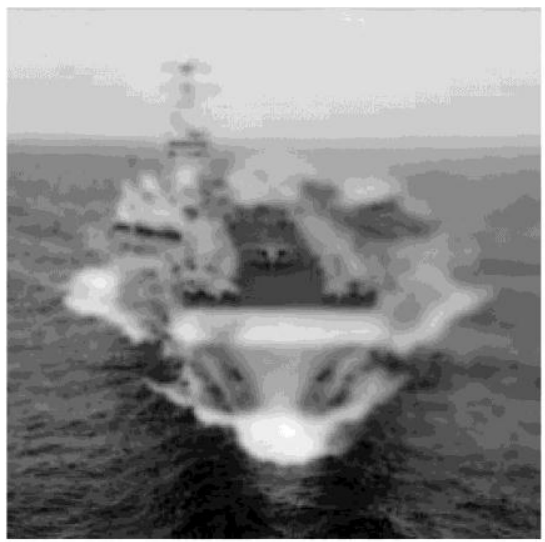

(I) Blur with $c=2.5, d=1.5$

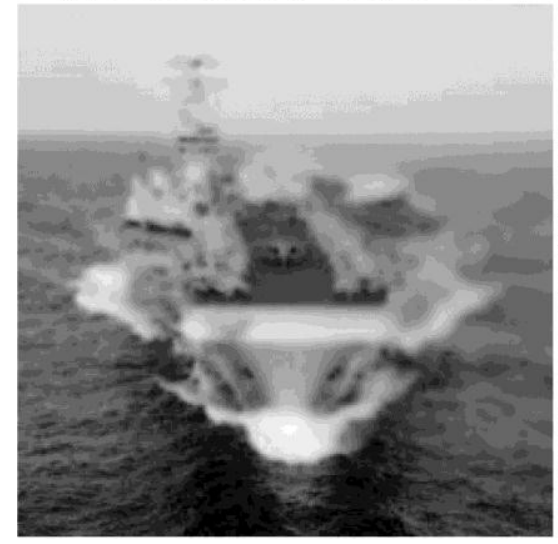

After 100 VanCittert iterns.

Figure 12. Nonlinearly blurred image $(H)$ was successfully deblurred after 100 interations. Visually indistinguishable image (I), blurred with stronger nonlinearities, could not be usefully deblurred. Evidently, how an image was blurred, and not just the amount of blur, is critical in nonlinearly blurred imagery.

linear, autonomous, selfadjoint canonical model. Additional difficulties may arise from the Van Cittert iteration, originally intended for linear convolution equations $S g=f$, where the kernel in $S$ has a positive Fourier transform.

The following nonlinear deblurring experiment illustrates the strengths and limitations of the above methodology. In Figure 11, a sharp image of the aircraft carrier USS Eisenhower is blurred, using Eq. (1) with two different sets of values for the constants $c$ and $d$. Because $d$ is larger in image (I) than it is in image $(\mathrm{H})$, the nonlinearity is stronger in image (I). However, as shown in Table 3, images (I) and (H) have almost the same values for their $L^{1}, L^{2}$, and $\mathrm{k}\|\nabla f\|_{1}$ norms. In addition, images (I) and (H) have identical PSNR val- ues, an image quality metric that measures the degradation away from the original sharp image (G). Thus, images (I) and (H) are visually and statistically indistinguishable in Figure 11.

In Figure 12, the nonlinearly blurred image $(\mathrm{H})$ is successfully deblurred after 100 iterations. This is a highly encouraging result, as there are very few pubpublished examples of successful nonlinear multi-dimensional backward parabolic continuations. Many other such examples, involving a variety of nonlinearities, are equally feasible.

The unsuccessful deblurring of image (I) suggests that if the nonlinearities are too strong in the parabolic equation, the resulting backward stability may be too weak to permit useful reconstruction. In addition, the Van Cittert iteration may become illbehaved, even if the blurred image is virtually indistinguishable from an image that can be usefully deblurred.

\section{References}

[1] C. Bagtzoglou and J. Atmadja, Marching-jury Backward Beam Equation and Quasireversibility Methods for Hydrologic Inversion: Application to Contaminant Plume Spatial Distribution Recovery, Water Resources Research 39 (2003), 1038-1052.

[2] A. S. Carasso, Bochner Subordination, Logarithmic Diffusion Equations, and Blind Deconvolution of Hubble Space Telescope Imagery and Other Scientific Data, SIAM Journal of Imaging Sciences 3 (2010), 954-980.

[3] A. S. Carasso, Reconstructing the Past from Imperfect Knowledge of the Present: Effective Non-uniqueness in Solving Parabolic Equations Backward in Time, Mathematical Methods in the Applied Sciences (2012), DOI: 10.1002/mma.2582.

[4] P. H. Van Cittert, Zum Einfluss der Spaltbreite auf die Intensittsverteilung in Spektrallinien II, Z. Physik 69 (1931), 298-308. 


\section{Metrics for Manipulation and Enhancement of Forensic Images}

Fingerprint evidence is routinely used to convict offenders of crimes, but such evidence is rarely pristine. Factors that influence the quality of latent fingerprint evidence collected from a crime scene include: incomplete prints, pressure gradations, variability in underlying surface and materials, including colors. Due to the low signal quality of the fingerprint in relation to other systematic image features (color, pattern, text etc.), these images are not suitable for input into fingerprint identification software to search for matches. In response to this problem the forensics community currently uses a variety of image processing tools to improve the quality of these images. To date, latent image enhancement has been treated as a preprocessing step for subsequent identity matching analysis. As dramatic and essential as these transformations are, no research exists to characterize this step of the evidentiary analysis chain. Detrimental consequences for reproducibility, traceability, and quantification of accuracy follow [2]. This project represents a first investigation of this step of the forensic analysis workflow.

\section{Andrew Dienstfrey}

In FY 2012 we initiated a new project to research and develop metrics to characterize image processing performed in the course of forensic analysis of latent fingerprint images. Previously this critical step in the evidentiary workflow has been insufficiently characterized, and its reproducibility has not been studied. This project will facilitate the creation of reproducible and quantitative analysis to promote a scientific basis for latent fingerprint enhancement. We expect that this project will provide a foundation for comparable studies in other forensic image analysis workflows.

To establish a controlled testbed, two expert forensic analysis companies-Schwarz Forensic Enterprises and ForayTechnologies - created a database of surrogate latent fingerprints and their associated enhancements. The images shown in Figure 13 are two examples from this new database. The original latent fingerprints are shown on the left, and the associated enhancements are shown to the right, Figure 13 (a), (c) and (b), (d) respectively. Visually, the result of the enhancement process is striking. While fingerprints can barely be seen in the original images, the combination of the removal of systematic background features and color processing reveals enhanced images that undeniably contain fingerprints. Schwarz and Foray initially provided 39 before-after pairs and associated metadata (see below). A second round consisting of more than 60 additional enhancement pairs has been provided and
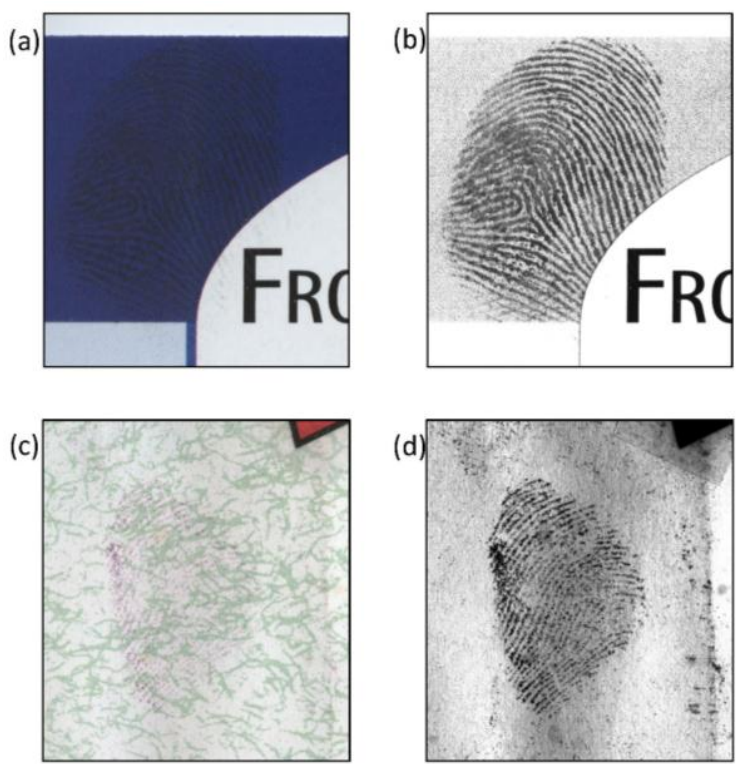

Figure 13. Sample latent prints in the NIST database and their Photoshop enhancements.

uploaded to NIST servers and will be analyzed in follow-up work.

Our first goal was to understand the scope of image transformations most often used to enhance latent prints. Forensic guidelines require that enhanced images be accompanied by metadata indicating all processing applied to an evidentiary article. Adobe Photoshop is the image enhancement software of choice in the forensic sciences as practiced today. Within Photoshop this metadata requirement is met by activating a logging utility that records the sequence of Photoshop commands executed by the analysts. All enhancements in the new NIST latent fingerprint archive are accompanied by such text files. This compendium provides valuable data for analysis of the nature of the enhancement processing. For example, a parser was written to extract command usage from the $\log$ files. A preliminary result on the frequency of command usage is shown in Figure 14. The space of image processing possibilities is quite large. Using a ranking-by-frequency approach this analysis represents the first systematic investigation of the relative importance of enhancement procedures of which we are aware.

In addition, this collection of $\log$ files enables us to begin addressing the question of enhancement reproducibility. In current forensic practice the metadata files are required as a mechanism for reproducibility of the enhancement process should it be questioned in an evidentiary proceeding. However, a comparative study of the Photoshop command structure and the details 


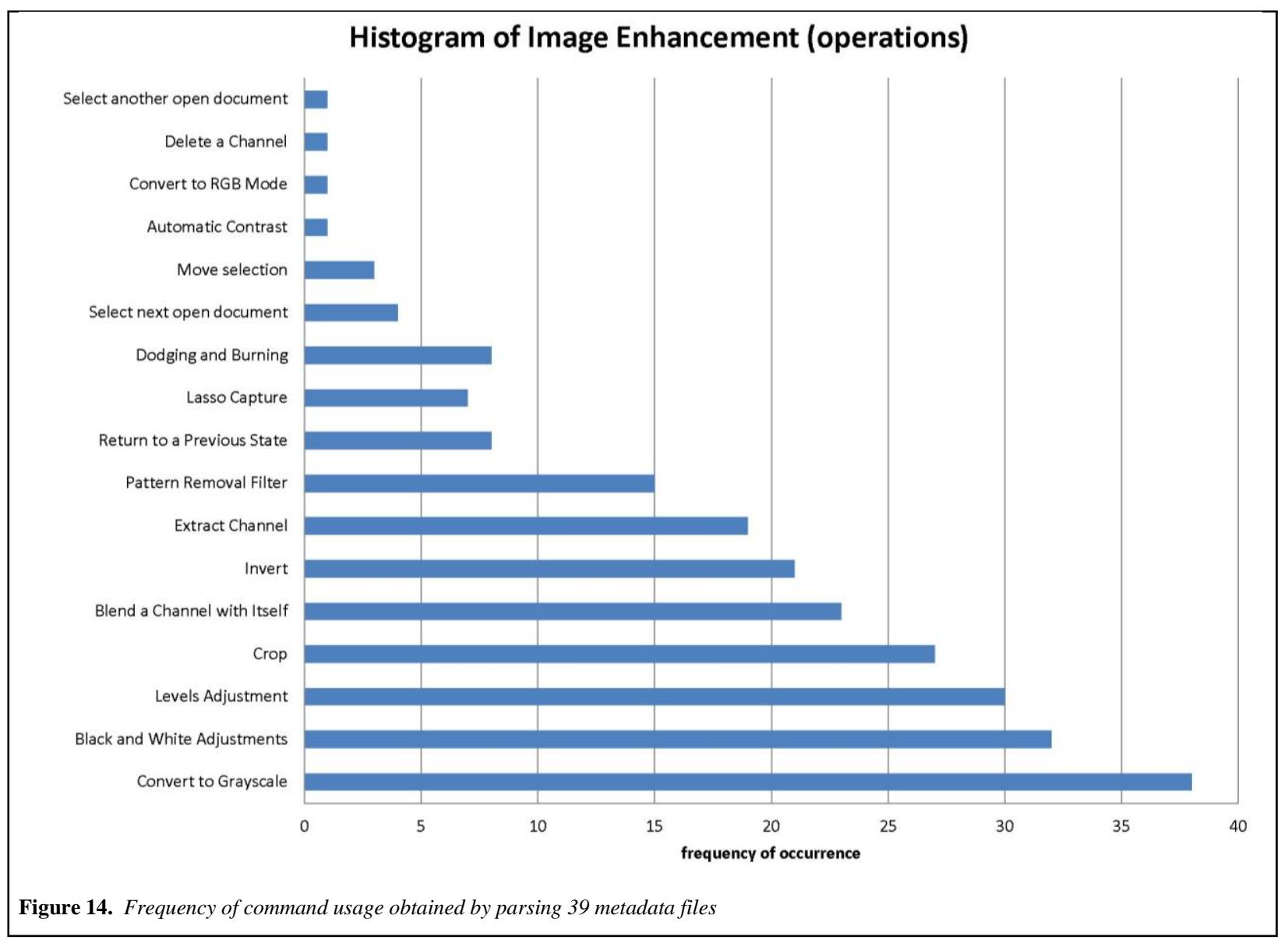

provided in the metadata logs reveals a substantial inadequacy in this regard. For example, in a preliminary analysis of 40 latent enhancements we find that combination of either burn and dodge commands is used over 120 times; indicative of frequent and repeated utilization. However, while in practice these commands require user-specified parameters in the form of bounding boxes and "strength" (i.e., of the lightening or darkening associated with the dodge or burn respectively), in the case of burn and dodge, the log files simply record the command name with no parameter information. It follows that pixel-by-pixel reproducibility is not possible using this record-keeping utility in the Photoshop enhancement workflow. This example, along with others, provides a basis for future research on details and implementation of reproducibility requirements in the forensic image enhancement workflow.

Finally we investigated possibilities for metrical analysis of the enhancements as well as alternative image enhancements performed independently of Photoshop. In Figure 15 we show an example of an enhancement performed using Matlab in comparison with the result achieved with Photoshop. Visually the resulting enhancements appear comparable. Consideration of such alternative enhancements may better inform the subsequent matching and identification procedure as they provide an idea of the range of images that may be reasonably obtained. In addition, using scientific packages such as Matlab, IDL, and PV-Wave provides additional benefits as they generally include a broader set of processing commands, yet do so in a more rigorous and reproducible manner.

Concerning the metrical analysis, among the many image metrics we are investigating we draw attention here to the discrete $L^{1}$ and $L^{2}$ image norms, as well as the discrete $L^{1}$ and $L^{2}$ image gradient norms. For an image $f(x, y)$ of size $N_{x}$ by $N_{y}$ these are defined by

$$
\begin{aligned}
& \|f\|_{p} \equiv\left(\frac{1}{N_{x} \cdot N_{y}} \sum_{x, y=1}^{N_{x}, N_{y}}|f(x, y)|^{p}\right)^{1 / p} \\
& \|\nabla f\|_{p} \equiv\left(\frac{1}{N_{x} \cdot N_{y}} \sum_{x, y=1}^{N_{x}-1, N_{y}-1}\left(\left(\nabla_{x} f(x, y)\right)^{2}+\left(\nabla_{y} f(x, y)\right)^{2}\right)^{p / 2}\right)^{1 / p} \\
& \nabla_{x} f(x, y) \equiv N_{x} \cdot(f(x+1, y)-f(x, y)) \\
& \nabla_{y} f(x, y) \equiv N_{y} \cdot(f(x, y+1)-f(x, y))
\end{aligned}
$$




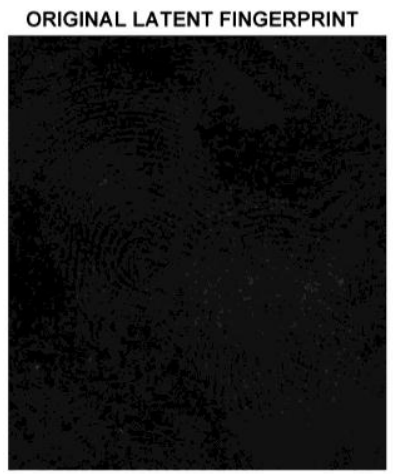

FORENSIC PHOTOSHOP PROCESS

MATLAB CONTRAST ADJUSTMENT
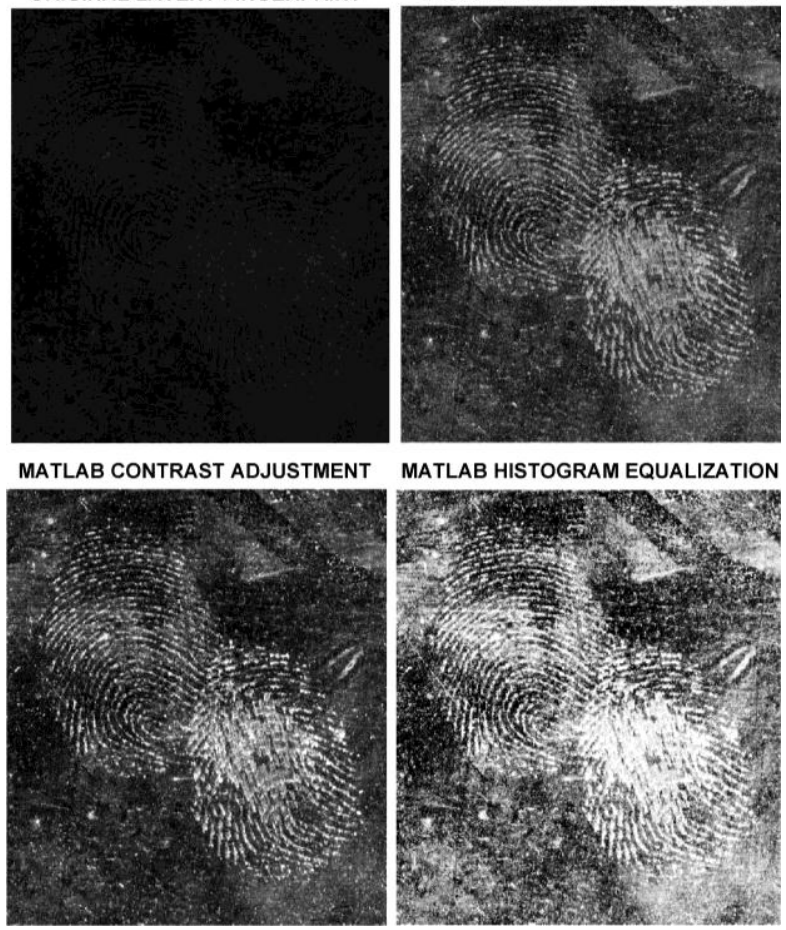

MATLAB HISTOGRAM EQUALIZATION

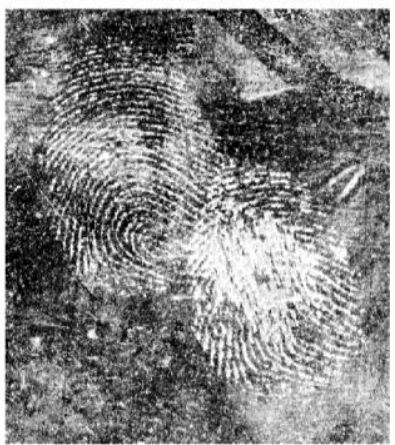

Figure 15. Sample enhancements performed independently of Adobe Photoshop.

Table 4. Result of measuring alternative enhancement workflows with different norms.

\begin{tabular}{|l|r|r|r|r|}
\hline Image & $\|f\|_{\mathbf{1}}$ & $\|f\|_{\mathbf{2}}$ & $\|\nabla f\|_{\mathbf{1}}$ & \multicolumn{1}{|c|}{$\boldsymbol{\nabla f \| _ { \mathbf { 2 } }}$} \\
\hline Original Laten & 16 & 18 & 2600 & 3900 \\
\hline Forensic Photoshop & 61 & 75 & 25000 & 36000 \\
\hline Matlab Contrast Adj. & 60 & 80 & 25000 & 40000 \\
\hline Matlab Histogram Eq. & 112 & 138 & 42000 & 60000 \\
\hline
\end{tabular}

Note that the gradient $L^{1}$ norm is a discrete version of the total variation (TV) norm frequently used in image analysis. Table 4 shows these norms applied to the four images in Figure 15. While the Photoshop and Matlab performed enhancements appear visually comparable, the $L^{2}$ image and gradient norms report different values for these two images. We also observe that the $L^{1}$ versions of these norms appear to be less discerning of this difference. We have performed similar analysis on several additional enhancements and find this pattern to be consistent. Specifically, the gradient $L^{2}$ norms routinely return different values, distinguishing diverse analysis workflows.

The above work has been documented in a NIST report [1]. In FY 2013 we expect to further investigate the metrical analysis using additional fingerprintspecific image metrics including the spectral image verification and validation described in [3].

This work was sponsored by a 2012 NIST Forensic Measurement Challenges grant.

\section{References}

[1] A. Carasso, Alternative Methods of Latent Fingerprint Enhancement and Metrics for Comparing Them, NISTIR 7910, National Institute of Standards and Technology, Gaithersburg, MD, January 2013.

[2] Expert Working Group on Human Factors in Latent Print Analysis, Latent Print Examination and Human Factors: Improving the Practice through a Systems Approach, NISTIR 7842, National Institute of Standards and Technology, Gaithersburg, MD, February 2012.

[3] J. M. Libert, J. Grantham, and S. Orandi. A 1D Spectral Image Validation/Verification Metric for Fingerprints, NISTIR 7599, National Institute of Standards and Technology, Gaithersburg, MD, 2009.

\section{Participants}

Andrew Dienstfrey, Alfred Carasso (ACMD); Mary Theofanos (NIST SCO), Peter Bajcsy (ITL SSD), Haiying Guan (ITL IAD), David Witzke (Foray Technologies), Matt Schwarz (Schwarz Forensic Enterprises) 


\section{Rheology of Dense Suspensions}

Understanding the mechanisms of dispersion or agglomeration of particulate matter in complex fluids, such as suspensions, is of technological importance in many industries such as pharmaceuticals, coatings, and construction. These fluids are disordered systems consisting of a variety of components with disparate properties that can interact in many different ways. Modeling and predicting the flow of such systems represents a great scientific and computational challenge requiring large-scale simulations. In collaboration with scientists in NIST's Engineering Laboratory (EL), we have developed an application, called QDPD (Quaternion-based Dissipative Particle Dynamics), which is capable of performing large-scale simulations of dense suspensions. QDPD is highly parallel and has been shown to efficiently scale up to at least 32,000 processors when running on the DOE supercomputer Intrepid at Argonne National Laboratory. Our goal in this project is to advance understanding of the flow properties of a specific material, fresh concrete, a dense suspension composed of cement, water, sand, and rocks.

Our current focus is on enabling the use of vane rheometers for measuring the flow properties of fresh concrete, something not currently possible. While different concrete flow measurements can be found to correlate, they usually do not agree well. A vane geometry combined with a strongly random suspension will result in complex local stress and strain/strain rate fields. Current vanes are not optimized for precise measurement (or mixers for best mixing practice). These simulations will enable us to map out stress/strain fields in $3 D$, and bring insight into the vane design process. In addition to the advancement of our understanding of the flow of dense suspensions, we are also using this simulator in the design process for new standard reference materials (SRMs) which will be used for calibrating mortar and concrete rheometers.

\section{William George}

Concrete is the most widely used building material in the world, representing a 100 billion dollar industry in the US that is crucial for our nation's physical infrastructure. There is now a strong interest in making concrete a more sustainable material by finding new ways to recycle it, and by changing its ingredients in order to reduce the amount of greenhouse gas from its production. (The manufacture of concrete's key ingredient, cement, is responsible for about $8 \%$ to $10 \%$ of global $\mathrm{CO}_{2}$ production.) As new mixture designs of concrete are developed to meet these needs, it is important to measure and control rheological (i.e., flow) properties to satisfy performance specifications. Failure
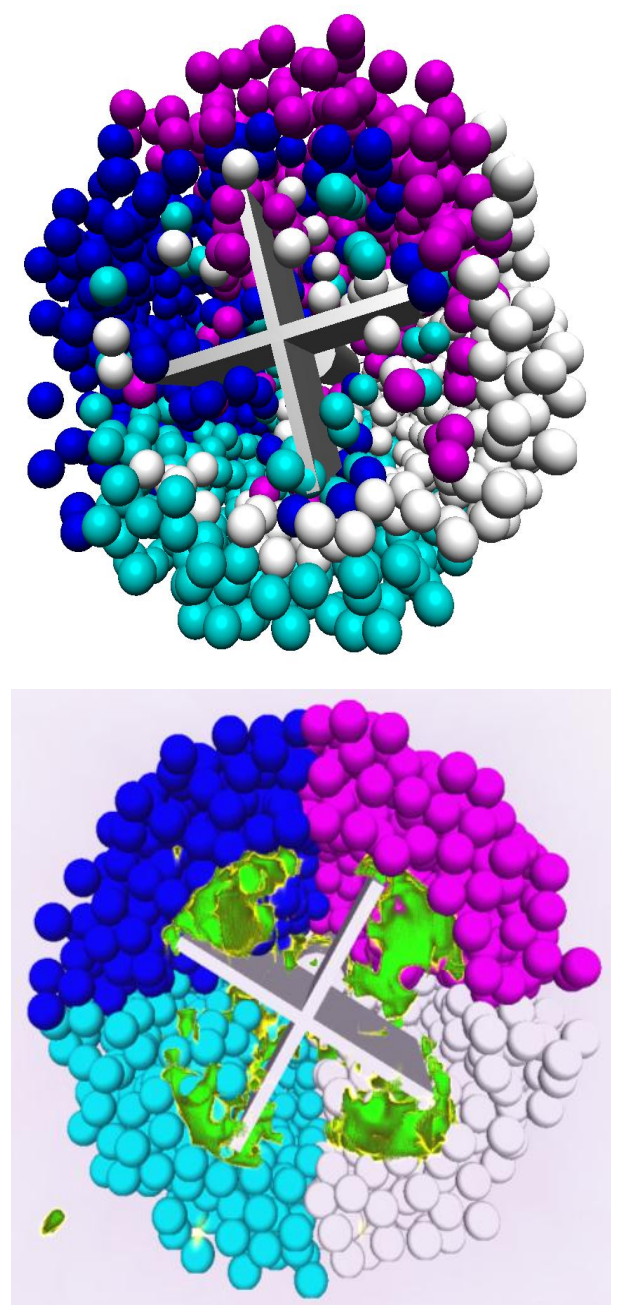

Figure 16. Top: Snapshot from a simulation of a four-blade vane rheometer with a suspension of hard spheres. Each sphere is colored-coded by the rheometer octant in which it was located at the start of the simulation. The blades are rotating clockwise and have made approximately two full rotations at the time of this snapshot. Bottom: Vane rheometer simulation displayed with a volume visualization of the magnitude of the local stress within the system. Only four of the eight octants are shown here in order to see details around the vanes. Here stress is color-coded from yellow (low stress), to green (high stress), with stresses below a set threshold show in light purple.

to control the flow of concrete on a job site can lead to significant cost overruns and months of delay by having to correct previous errors.

Often fluids are characterized by the properties yield stress and viscosity, both of which can vary as a function of shear rate. Yield stress is the force applied per unit area to initiate the flow. Viscosity is the applied force per unit area needed to maintain a shear rate. Shear rate is the velocity gradient perpendicular to the flow direction. An image showing the local stress in a vane rheometer is shown in Figure 16. 


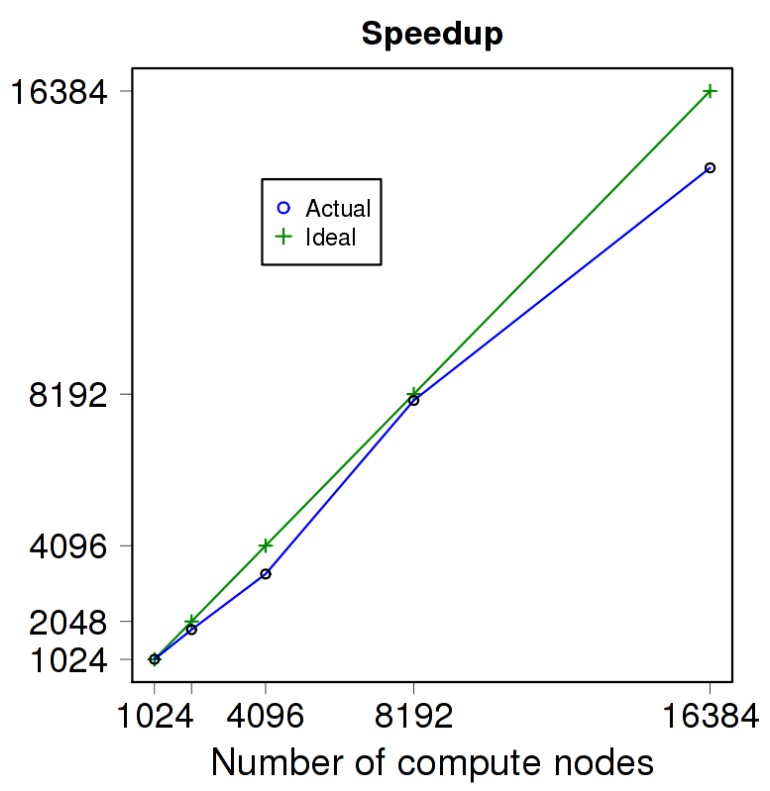

Figure 17. Performance of QDPD on Intrepid, an IBM Blue Gene/P, on a system of approximately $10^{5}$ inclusions (rocks) and $10^{8}$ DPD particles.

Many factors control viscosity and yield stress. For example, in building materials such as concrete, viscosity and yield stress depend on the ratio of water to cement, the volume of sand or rocks used, as well as their shape and size distribution. There can be great variation in each material depending on its history and where it was obtained. For example, rocks from quarries are usually angular because they are crushed when processed, whereas rocks obtained from river beds are typically rounded due to erosion. Additionally, it turns out that the more similar the size of the rocks in a concrete suspension, the harder it is to get that concrete to flow. In this case, the concrete may actually jam when poured through a narrow opening, thus causing delays in construction. Clearly, to optimize the flow properties of concrete and other suspensions, one needs to understand the relationship between flow and properties of the fluid's constituents.

Quaternion-based Dissipative Particle Dynamics. Modeling and predicting the flow of suspensions such as concrete represents a great scientific challenge, since these are complex disordered systems with a variety of components that can interact in many different ways. Indeed, accounting for the size and shape variation of the solid components of the suspension (cement particles, sand, or rocks) poses many computational challenges. A representative system may entail keeping track of up to 100,000 solids of varying shape and size. Further, many of the forces between particles depend on the local surface curvature of the aggregate at points close to neighboring aggregates, which requires keeping track of the location, orientation and shortest distance between neighboring solids. Clearly, modeling such systems necessitates large scale simulations at a fundamental level to accurately predict its properties. We have adopted and developed some novel approaches, originally based on cellular automata methods that can successfully take into account many of the features of a suspension. Our code, QDPD, has been validated by both theory and experiments on idealized systems and has been extended to account for random shaped objects with different interparticle interactions.

QDPD [1], which stands for Quaternion-based Dissipative Particle Dynamics (DPD), uses modified versions of the DPD technique [2] for the simulation of Newtonian fluids, and the Smoothed Particle Hydrodynamics technique (SPH) [3] for the simulation of nonNewtonian fluids, in order to simulate dense suspensions such as cement paste, mortar, and concrete. In addition to the forces computed using the DPD and SPH techniques, other forces are computed in these systems to better account for the interaction between the suspended particles, that is, the sand and gravel. These additional forces include lubrication forces that help keep these suspended particles separated, and van der Waals forces that introduce an attractive interparticle force. Brownian forces are also present in QDPD to maintain system temperature.

High Performance Computing. Starting with the original serial version of this simulator we have enhanced QDPD to utilize the power of large parallel machines regularly using $8000+$ processors, and, for large production runs, using 32,000 processors or more. The performance of QDPD running a typical simulation on an IBM Blue Gene/P is shown in Figure 17 in terms of speedup [8]. QDPD remains under constant development to improve its capabilities as well as to improve its parallel performance. For example, we have recently begun basic algorithm changes to enable the efficient execution of our simulations on at least 170,000 processors of Mira, the latest DOE machine at the Argonne Leadership Computing Facility (ALCF). This includes changes in inter-processor communications as well as the addition of multi-threading.

During the 2012 calendar year we have had access to the IBM Blue Gene/P supercomputer Intrepid, having been awarded 22 million CPU-hours of compute time on this machine from the DOE INCITE program (Innovative and Novel Computational Impact on Theory and Experiment). This access has enabled us to perform several very large simulations of a 4-blade vane rheometer. We have been awarded a third year of compute time, for 2013, at ALCF through our latest peer-reviewed INCITE proposal. For 2013 we will have a total of 30 million CPU-hours of compute time at the ALCF, including 10 million CPU-hours of time on Mira, the new IBM Blue Gene/Q at Argonne. Mira, which consists of 786,432 processors, and is ranked 


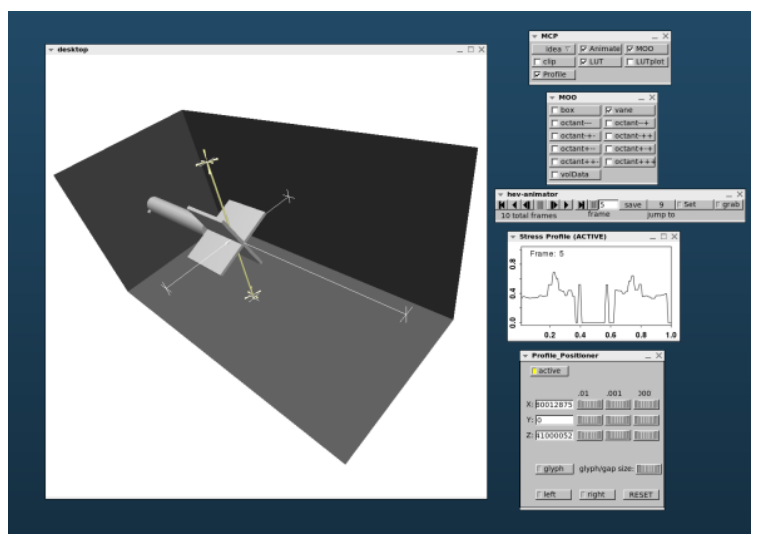

Figure 18. Interactively positioning a line probe in a system. Each endpoint is indicated with a $3 D$ cursor consisting of three orthogonal pairs of small cones surrounding the chosen point. The endpoints of this line probe can be positioned anywhere within the simulation box shown in this image. The hard spheres and volume visualization of the stress have been turned off for this image in order to highlight the probe.

number 4 on the November 2012 Top 500 Supercomputers list ${ }^{11}$.

Visualization. A major part of this project involves the development and use of specialized visualization software to investigate in detail the results of our simulations. This includes both the display of the systems under study as well as techniques to probe and measure aspects of the system we are viewing. For performance reasons we rely heavily on the programmable capabilities of GPUs (Graphics Processing Units). For example, within the images in each of the figures, each suspended sphere is rendered within the GPU as a point sprite, i.e., a 2D textured image at a single 3D point, which requires only location and size information for display, rather than using a polygonal representation of these spheres. We also use volume visualization techniques to display various properties within the system, such as in Figure 16 (bottom) which shows the local stress value throughout the system. As shown in Figure 18 and Figure 19, we can interactively probe a system to reveal values of properties at specific locations or areas in the system. All of our visualizations can also be used within our 3D immersive visualization system for full immersion into the simulated systems. This system consists of 3 large displays (each approximately $2.5 \mathrm{~m}$ by $2.5 \mathrm{~m}$ ), arranged as two walls and a floor forming the corner of a cube.

Results. Over the last few years our simulations have provided fundamental insights into the mechanisms that control the onset of flow in suspensions that can be linked to macroscopic properties such as yield stress and viscosity [4, 7]. Our studies have also enabled us to discover that, given the rheological properties of a nonNewtonian fluid matrix in a dense suspension, such as

11 http:// www.top500.org/ a mortar or concrete, we can predict key rheological properties. For example, we have simulated the flow of dense suspensions consisting of $1 \mathrm{~mm}$ diameter glass beads suspended in a matrix fluid of known properties, NIST SRM 2492 [5], for volume fractions ranging from $10 \%$ to $49 \%$ glass beads. These simulations have been performed for a large range of shear rates. From our studies, we have discovered that, given the rheological properties of a non-Newtonian fluid matrix, we can predict the rheological properties of suspensions composed of hard spheres in this fluid [6]. We are currently writing a paper detailing this discovery. Such suspensions are currently being evaluated as a new candidate SRM for mortars. As a consequence of this discovery, one only needs to measure the fluid properties of the matrix fluid, which is generally far easier to do than measuring the suspension. Hence, fewer experiments are needed to obtain the rheological properties of the suspension, saving cost and time for industry. Currently these results apply only to hard sphere suspensions. However, we plan to test this scaling approach on suspensions composed of more complex shaped inclusions.

Finally, while the main thrust of our research focuses on predicting the rheological properties of cement based materials such as concrete, an improved general understanding of rheological properties derived from this research should have a broad impact. Suspensions are utilized in a wide variety of technological processes and, as our study is largely parametric in nature, results will be transferable to other suspensions of interest such as nanoparticle systems. Understanding mechanisms for the dispersion or agglomeration of such systems remains a challenge in many industries, ranging from pharmaceuticals to coatings.

\section{References}

[1] N. Martys, Study of a Dissipative Particle Dynamics Based Approach for Modeling Suspensions, Journal of Rheology 49 (2) (2005).

[2] P. J. Hoogerbrugge and J. M. V. A. Koelman, Simulating Microscopic Hydrodynamic Phenomena with Dissipative Particle Dynamics, Europhysics Letters 19 (1992), 155-160.

[3] J. J. Monaghan, Smoothed Particle Hydrodynamics. Reports on Progress in Physics 68 (2005), 1703-1759.

[4] N. S. Martys, M. Khalil, W. George, D. Lootens, and P. Hebraud, Stress Propagation in a Concentrated Colloidal Suspension under Shear, The European Physical Journal E 35 (3) (2012), 20 (7 pages).

[5] C. F. Ferraris, P. Stutzman, J. Winpigler, and W. F. Guthrie, Certification of SRM 2492: Bingham Paste Mixture for Rheological Measurements, NIST Special Publication SP 260-174 Rev. 2012, U.S. Government Printing Office: Washington, DC (2012).

[6] D. Lootens, N. Martys, and P. Hebraud, Universal 
Behaviour of Non-Newtonian Suspensions, $\mathrm{XVI}^{\text {th }}$ International Conference on Rheology, Lisbon, Portugal, 05-Aug-2012.

[7] P. Hebraud, D. Lootens, and N. Martys, Stress Organization in an Attractive Concentrated Colloidal Suspension under Flow, $\mathrm{XVI}^{\text {th }}$ International Conference on Rheology, Lisbon, Portugal, 05-Aug2012.

[8] W. George, N. Martys, C. Ferraris, S. Satterfield, M. Olano, and J. Terrill, Computational Based Study of Suspension Flow in Vane Rheometers, Workshop on High-Performance Computing for Industry, Rensselaer
Polytechnic Institute, Troy, New York, October 26-38 (2011).

\section{Participants}

William George, Steven Satterfield, Marc Olano, Judith Terrill (ACMD); Nicos Martys, Edward Garboczi (NIST EL); Pascal Hebraud (CNRS/ESPCI, France).

http://www.nist.gov/itl/math/hpcvg/rheology.cfm http://www.nist.gov/itl/math/hpcvg/concretevis.cfm

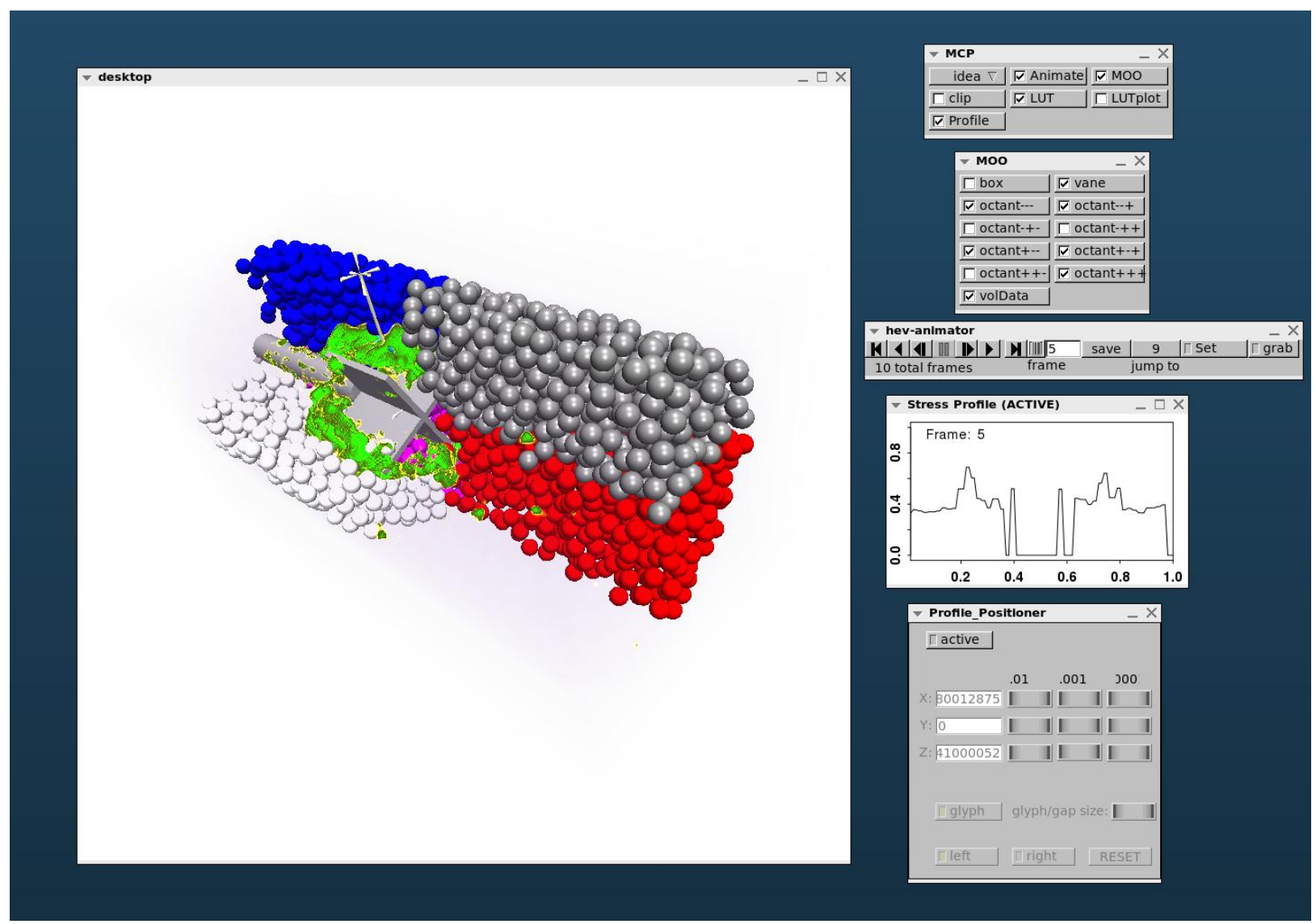

Figure 19. This is the identical display as in Figure 18 except that now we have turned on the volume visualization of the stress data as well as some of the hard spheres. The stress along the probe line is shown in the sub-window labeled Stress Profile. This plot is dynamically generated and updates each frame as the simulation is animated. 


\section{Visualization and Modeling of RF Propagation in Body Area Networks}

\begin{abstract}
A body area network (BAN) is a radio communication and networking protocol for short range, low power and highly reliable wireless connectivity for use inside or around a human body. It includes radio frequency $(R F)$ enabled devices that could be implanted, worn or located adjacent to the body. Radio-enabled implantable sensor nodes offer a revolutionary set of applications, among which are smart pills for precision drug delivery, glucose monitors, blood pressure monitors, and eye pressure sensors for glaucoma patients. At the same time, wearable sensor nodes can enable remote wireless monitoring of electrocardiogram $(E C G)$, temperature, respiration, and heart rate. While $B A N$ is poised to be a promising technology with novel uses in pervasive healthcare, there are still numerous technical, commercial and regulatory challenges, including usability, interoperability, energy source, miniaturization, security, and privacy. One of the prerequisites for the safe and effective design of such systems is an understanding of RF propagation around and inside the body. We have developed a visualization platform for the study of human body RF propagation and are applying it in support of IEEE standardization efforts.
\end{abstract}

\section{Kamran Sayrafian}

With recent advances in microelectronics, the technology to build very small and extremely low power wearable and implantable devices is clearly within our reach. However, commercial success of this technology depends on the widespread adoption of a global standard for its communication protocol (i.e., air interface). Knowledge of RF propagation is a critical step in this process, enabling RF engineers to optimize their physical layer design to achieve better communication performance. Such information is typically gathered by conducting physical experiments and processing the measured data to obtain propagation channel characteristics. Obtaining data sufficient to study this for various scenarios is difficult for wearable BAN sensors. Moreover, obtaining any data through physical experimentation is extremely challenging to impossible for implantable devices.

In view of the above, a 3D modeling and visualization platform that is capable of emulating physical experiments can be extremely beneficial in understanding the radio waves propagation behavior in body area networks. Although validation with physical experiment is ultimately needed to investigate the reliability of the results, such a platform can be used to determine

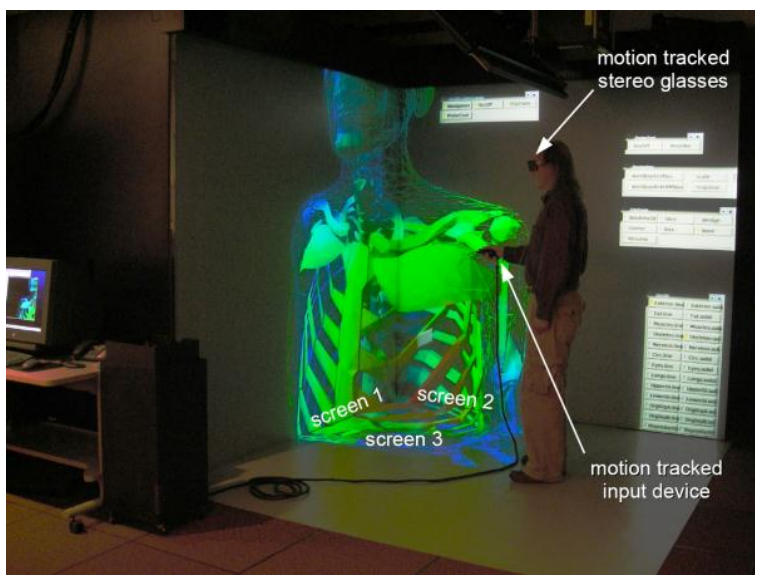

Figure 20. Our 3D-immersive platform for the study of RF propagation in body-area networks.

the best (i.e., optimized) test scenarios for more limited physical experiments.

For this reason, we have set up a unique 3D immersive visualization environment that allows virtual experiments to be performed in order to study various RF propagation scenarios among implantable or wearable body sensors. Inhomogeneity and complexity of the propagation medium, along with possible propagation paths from any direction, necessitate a 3D environment to better capture, visualize, and understand RF propagation. The complexity of the environment surrounding a medical implant also implies that an appropriately designed immersive platform would be very helpful in better understanding the radio frequency channel characteristics inside the human body. Lack of a sufficiently detailed human body model and realistic wearable/implantable antenna models are usually among the shortcomings of previous simulation studies in this area. The present work overcomes these limitations.

Our 3D immersive platform, as shown in Figure 20, includes several components: a visual display consisting of three large screens, stereoscopic glasses that are motion-tracked, and a hand-held input device that is also motion-tracked. The three large projection screens are arranged edge-to-edge in a corner configuration; these are used as a single 3D stereo display. The 3D scene is updated for the position of the stereo glasses given by the motion tracker. This enables system to present a virtual 3D world within which the user can move and interact with the virtual objects; this is the essence of the immersive experience. A hand-held three button motion-tracked wand with a joystick is the principal interaction device. 


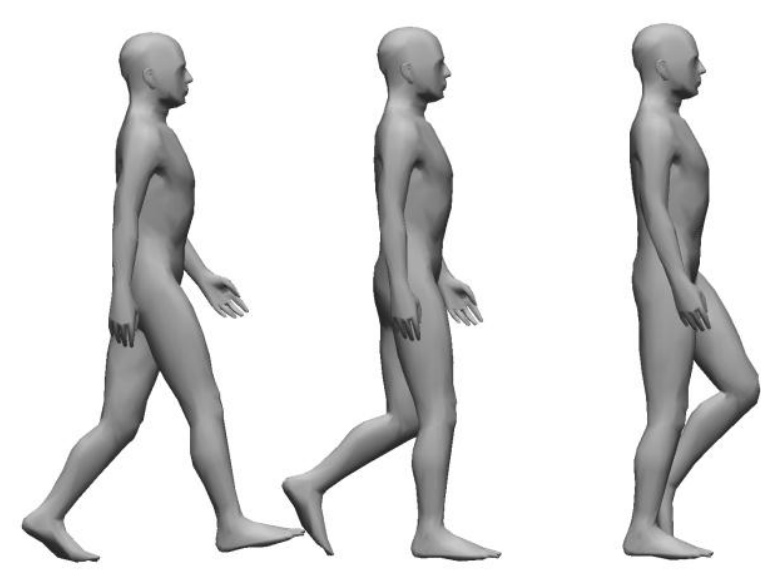

Figure 21. Several body poses from a walking sequence.

In this environment, a researcher can place a custom designed antenna at the desired location of the human body, set the operating frequency of the node, and study the RF propagation at any location inside or around the body. The platform user can observe the data representations at his desired scale and position. The user can physically move through data, and change visual orientation. The user can also control the elements of the virtual world using a variety of interactive measurement and analysis techniques. All of these capabilities are especially useful for the 3D study of RF propagation to/from implanted or wearable body devices. In addition, a more convenient and natural interaction between experts with different backgrounds is possible using this system. For example, a surgeon can point to the exact location of an implant using the interactive tools, and then an antenna designer can design the antenna to fit the chosen location, given the physical and biological constraints. An RF engineer, in turn, would be able to observe the propagation performance for the target communication link, or compute other measures, such as specific absorption rate (SAR), given the transmitted power.

Some of our results that are related to the statistical modeling of path-loss for implant communication have been adopted by the IEEE 802.15.6 International standard on body area networks [1]. In [2], we have explained how our simulation platform can be used to better understand the discrepancies between various sets of physical measurements related to body surface propagation. The impact of an aortic valve on this type of propagation has also been determined using our platform [3]. Evaluating the efficiency of custom made antennas and studying the creeping wave propagation on the body surface can be easily done in our system as well [4]. In addition to qualitative and quantitative data observation and statistical processing, studying RF propagation for specific medical applications such as cochlear implants, brain neural interface and retina implants is also possible using such platforms.

We are currently studying the effects of body pose and motion on RF propagation in BAN. This is referred to as dynamic channel and it is a more realistic scenario of operation of wearable and implantable sensors in a human body. To accomplish this, we have developed computational methods for deforming the 3D body model (including the internal body organs) into various poses. The most difficult problem that we had to solve in the body deformation was to ensure that the deformed internal structures do not intersect one another, thereby rendering the model invalid for our simulations. We plan to use these methods to create a sequence of poses to describe a motion such as walking. Figure 21 shows three poses from a sequence that describes a walking motion. The posed body models are then used as the basis for a series of simulations. Through collaborative efforts with other research institutes, we plan to compare the results of our simulations with experimental results. We anticipate that these efforts will accelerate the commercial development of BAN technology along with its related standards.

\section{References}

[1] K. Sayrafian-Pour, W. Yang, J. Hagedorn, J. Terrill, K. Y. Yazdandoost, A Statistical Path Loss Model for Medical Implant Communication Channels, in Proceedings of the IEEE International Symposium on Personal, Indoor and Mobile Radio Communications, 2009.

[2] W. Yang, K. Sayrafian-Pour, J. Hagedorn, J. Terrill, K. Y. Yazdandoost, Simulation Study of Body Surface RF Propagation for UWB Wearable Medical Sensors, in Proceedings of the 2nd International Symposium on Applied Sciences in Biomedical and Communication TechTechnologies, November 2009.

[3] W. Yang, K. Sayrafian, J. Hagedorn, J. Terrill, K. Yazdandoost, A. Taparugssanagorn, M. Hämäläinen, J. Iinatti, Impact of an Aortic Valve Implant on Body Surface UWB Propagation: A Preliminary Study, in ProProceedings of the 5th International Symposium on Medical Information and Communication Technology (ISMICT), March 2011.

[4] K. Sayrafian, J. Hagedorn, W. Yang, J. Terrill, A Virtual Reality Platform to Study RF Propagation in Body Area Networks, in Proceedings of the $3^{\text {rd }}$ IEEE International Conference on Cognitive Info-communications, December 2012.

\section{Participants}

John Hagedorn, Kamran Sayrafian, Judith Terrill

http://www.nist.gov/itl/math/hpcvg/ban.cfm 


\section{A Stochastic Theory of Fatigue for Aging Structures}

\begin{abstract}
Engineering components and structures are designed to function without premature failures due to aging. Traditionally, for new structures, their useful lives have been estimated from mathematical models of fatigue which are deterministic. Those models contain parameters that are determined from experiments using specimens of the structure when new. It is well-known that as structures age, their properties change, and hence the models based on the original materials are no longer applicable. In other words, it is not correct to simulate the performance of the aging structures when their current properties are not known. To address this gap-in-modeling problem, we develop a stochastic theory of fatigue, using in-situ nondestructive evaluation (NDE) measurements of detectable crack lengths and crack growth rates in an aging component or structure as a basis for estimating the mean and standard deviation of its remaining life.
\end{abstract}

\section{Jeffrey Fong}

It has been almost fifty years since Paris [1] proposed a simple model to describe the crack growth behavior of a large number of engineering materials by a relationship between cyclic crack growth rate, $d a / d N$ and stress intensity range $\Delta K$,

$$
d a / d N=C(\Delta K)^{m}
$$

where $a$ is the crack length, $N$ is the number of cycles, and $C$ and $m$ are two material constants that are estimated from a log-log plot of test data (after Paris, et al. [2]). The model has since been widely accepted (see, e.g., Kanninen and Popelar [3], Dowling [4]). In particular, it has been adopted in the ASME Boiler and Pressure Vessel Code for fatigue design of new and nondestructive evaluation (NDE) of aging pressure vessels and piping (see, e.g., Bamford [5]).

It turns out that the constant $C$ is a pseudo material property parameter, because it is sensitive not only to the cyclic stress ratio $R\left(=s_{\min } / s_{\max }\right)$, as shown by Dennis [6] for a constant amplitude cyclic stress test with the stress range $\Delta S$ defined by $\left(s_{\max }-s_{\min }\right)$, but also to three additional empirical material parameters as proposed by Walker [7] and documented by Dowling [4, p. 506, Table 11.2]. In spite of these complications in modeling fatigue crack growth, engineers have devised a formula for estimating the useful life (in cycles), $N_{i f}$, of a new component or structure by integrating Eq. (1):

$$
N_{i f}=\frac{a_{f}^{1-m / 2}-a_{i}^{1-m / 2}}{C(F \Delta S \sqrt{\pi})^{m}(1-m / 2)}
$$

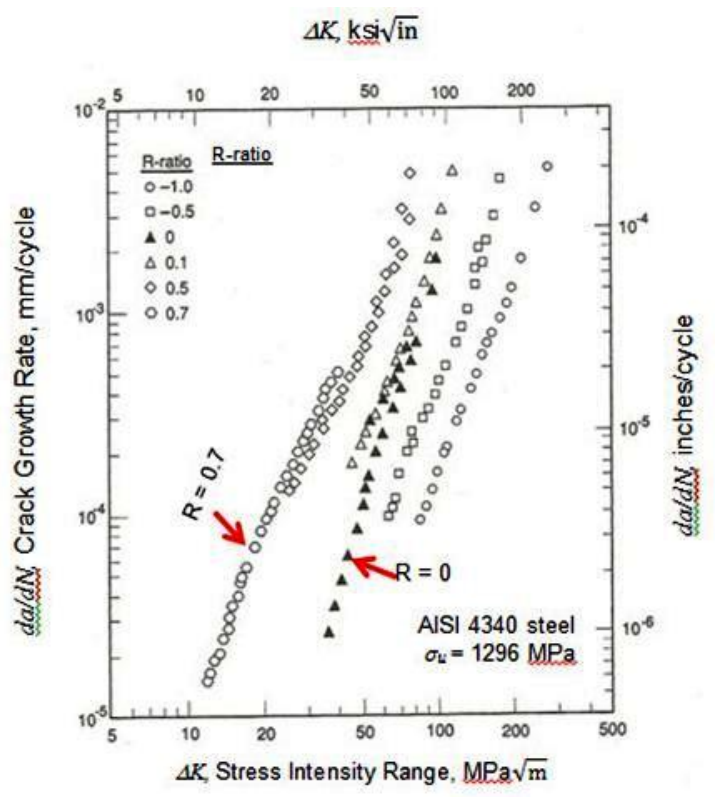

Figure 22. Effect of R-ratio on crack growth rates for an alloy steel. For $R<0$, the compressive portion of the load cycle is here included in calculating $\Delta K$. (After Dowling [4, p.494] with data from Dennis [6]).

where the third constant, $F$, is a geometric factor that is introduced to account for a reduction in the estimated life, $N_{i f}$, when the crack tip is located too close to a boundary. It is important to note that neither the initial nor final crack lengths $\left(a_{i}\right.$ and $\left.a_{f}\right)$ are measurable by NDE methods. As shown in Figure 22 (after Dowling [4, p. 49]), what is measurable by an NDE team is $a_{d}$, the minimum crack size that can be "reliably" detected, where $a_{d}>a_{i}$.

Another interesting material property parameter, known as the critical crack length, $a_{c}$, is also indirectly measurable, because it is the length that causes a specimen or full-scale structure to fail, and can be estimated from a formula (see Dowling [4, p. 521, Eq. 11.33]) based on the theory of brittle fracture. If a material is ductile, the final crack length, $a_{f}$, is always greater than $a_{c}$. In other words, $a_{f}>a_{c}>a_{d}>a_{i}$.

For most metals and alloys, Eq. (2) can be simplified by dropping the term involving the quantity $a_{f}$ using the following argument. Assuming we are working with a component made of AISI 4340 steel with crack growth data given in Figure 22. For $R=0.7$, Dowling [4, p.507] estimated that, for Eq. (2), the constant $C=2.49 \times 10^{-9}$, and $m=3.46$. From a plot in Dowling [4, p. 301, Fig. 8.12], and assuming that the crack tip is far from a boundary, we estimate that the geometric constant $F$ in Eq. (2) is approximately equal to 1.0. Let the maximum stress, $s_{\max }$, be equal to 300 MPa. With $R=0.7$, the minimum stress, $s_{\text {min }}$, is equal 


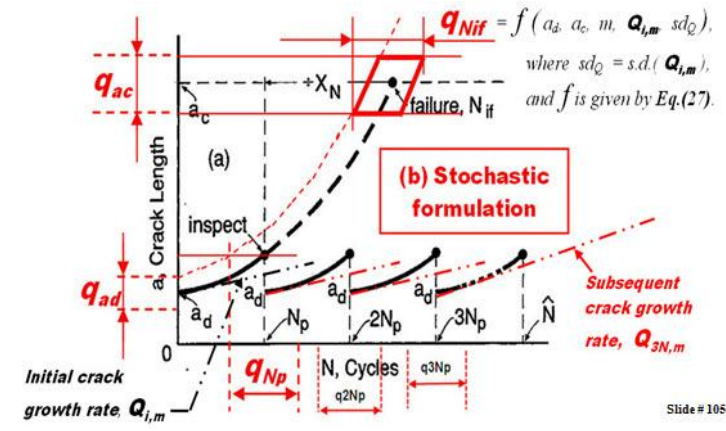

Figure 23. An application of the stochastic single-crack-growth theory (after Fong, et al. [8, 9]).

to $0.7 \times 300=210 \mathrm{MPa}$. The stress range, $\Delta S$, is, therefore, equal to $300-210=90 \mathrm{MPa}$. Let us assume, based on best estimates from the literature (see Kitagawa and Suzuki [10], and also Nisitani and Takao [11]), that the initial crack length, $a_{i}$, is of the order of $0.1 \mathrm{~mm}$. Let us also assume that the final crack length, $a_{f}$, equals $50 \mathrm{~mm}$. Substituting all of the above values of $C, m, F, \Delta S, a_{i}$, and $a_{f}$, into Eq. (2), we obtain $N_{i f}$ $=193$ cycles. If we drop the term involving $a_{f}$, we obtain $N_{i f}=197$ cycles, a difference of about $2 \%$.

Motivated by the large scatter of fatigue test data at microstructural, specimen, and full-scale structural levels, we have applied the classical theory of error propagation (see $\mathrm{Ku}[12]$ ) to the simplified Eq. (2) and developed a stochastic single-crack-growth theory of fatigue [9], as illustrated in Figure 23 and the following equations. (Note that $\eta^{\prime}=\left(\eta^{1-m / 2}-1\right) /(1-m / 2)$, in which $\eta=a_{f} / a_{i}$. )

$$
\begin{gathered}
N_{i f}=\eta^{\prime} \frac{a_{i}}{Q_{i, m}} \\
\operatorname{Var}\left(N_{i f}\right)=\left(\eta^{\prime}\right)^{2} \frac{a_{i}^{2}}{Q_{i, m}^{2}}\left[\frac{\operatorname{Var}\left(a_{i}\right)}{a_{i}^{2}}+\frac{\operatorname{Var}\left(Q_{i, m}\right)}{Q_{i, m}^{2}}\right]
\end{gathered}
$$

where

$$
Q_{i, m}=\left[\frac{d a}{d N}\right]_{a=a_{i}}
$$

\section{References}

[1] P. C. Paris, The Fracture Mechanics Approach to Fatigue, in Proceedings on the Symposium on FatigueAn Interdisciplinary Approach (J. J. Burke, et al., eds.), $10^{\text {th }}$ Sagamore Army Materials Research Conference, Syracuse University Press (1964), 107-127.

[2] P. C. Paris, et al., Extensive Studies of Low Fatigue Crack Growth Rates in A533 and A508 Steels, in Stress Analysis and Growth of Cracks, Proceedings of 1971 National Symposium on Fracture Mechanics, Part 1, ASTM STP 513, ASTM (1972), 141-176.
[3] M. F. Kanninen, and C. H. Popelar, Advanced Fracture Mechanics, Oxford University Press (1985).

[4] N. E. Dowling, Mechanical Behavior of Materials, $2^{\text {nd }}$ Edition, Prentice-Hall (1999).

[5] W. H. Bamford, Chapter 31. Fatigue Crack Growth, Fatigue, and Stress Corrosion Crack Growth: Section XI Evaluation, in Companion Guide to the ASME Boiler \& Pressure Vessel Code, Vol. 2, (K. R. Rao, ed.), 3rd edition, ASME Press (2009), 535-567.

[6] K. R. Dennis, Fatigue Crack Growth of Gun Tube Steel under Spectrum Loading, MS Thesis, Virginia Polytechnic Institute and State University (1986).

[7] K. Walker, The Effect of Stress Ratio during Crack Propagation and Fatigue for 2024-T3 and 7075-T6 Aluminum, in Proceedings of the Symposium on Effects of Environment and Complex Loading History on Fatigue Life, ASTM STP 462, ASTM International, West Conshohocken, PA (1970), 1-14.

[8] J. T. Fong, P. V. Marcal, O. F. Hedden, Y. J. Chao, and P. S. Lam, A Web-based Uncertainty Plug-In (WUPI) for Fatigue Life Prediction Based on NDE Data and Fracture Mechanics Analysis, in Proceedings of the ASME Pressure Vessels \& Piping Conference, July 2630, 2009, Prague, ASME, New York, NY (2009), Paper PVP2009-77827.

[9] J. T. Fong, N. A. Heckert, and J. J. Filliben, A Stochastic and Local Theory of Fatigue for Aging Pressure Vessels \& Piping, in From Failure to Better Design, Manufacture and Construction, (S. T. Tu, Z. D. Wang, and G. C. Sih, eds.), Proceedings of the $3^{\text {rd }}$ International Symposium on Structural Integrity, Oct. 31 - Nov. 4, 2012, Shandong University, Jinan, China, East China University of Science and Technology Press, Shanghai, China (2012), 19-31.

[10] H. Kitagawa, and I. Suzuki, in Reliability Approach in Structural Engineering, (A. M. Freudenthal, et al., eds.), Maruzen, Tokyo, Japan (1975), 217-233.

[11] H. Nisitani, and K. Takao, Significance of initiation, Propagation ad Crack Closure of Micro-cracks in High Cycle Fatigue of Ductile Metals, Engineering Fracture Mechanics 15 (3-4) (1981), 445-456.

[12] H. H. Ku, Notes on the Use of Propagation of Error Formulas, Journal of Research of the National Bureau of Standards - C. Engineering and Instrumentation, 70C (4) (1966), 263-273.

\section{Participants}

Jeffrey T. Fong (ACMD); N. Alan Heckert, James J. Filliben (ITL SED), Richard A. Fields, Stephen W. Freiman (NIST MML); Pedro V. Marcal (MPACT Corp., Oak Park, CA); Y. J. (Bill) Chao (University of South Carolina); Poh-San Lam (DOE Savannah River National Laboratory); Owen F. Hedden (ASME Codes $\&$ Standards) 


\section{Part III}
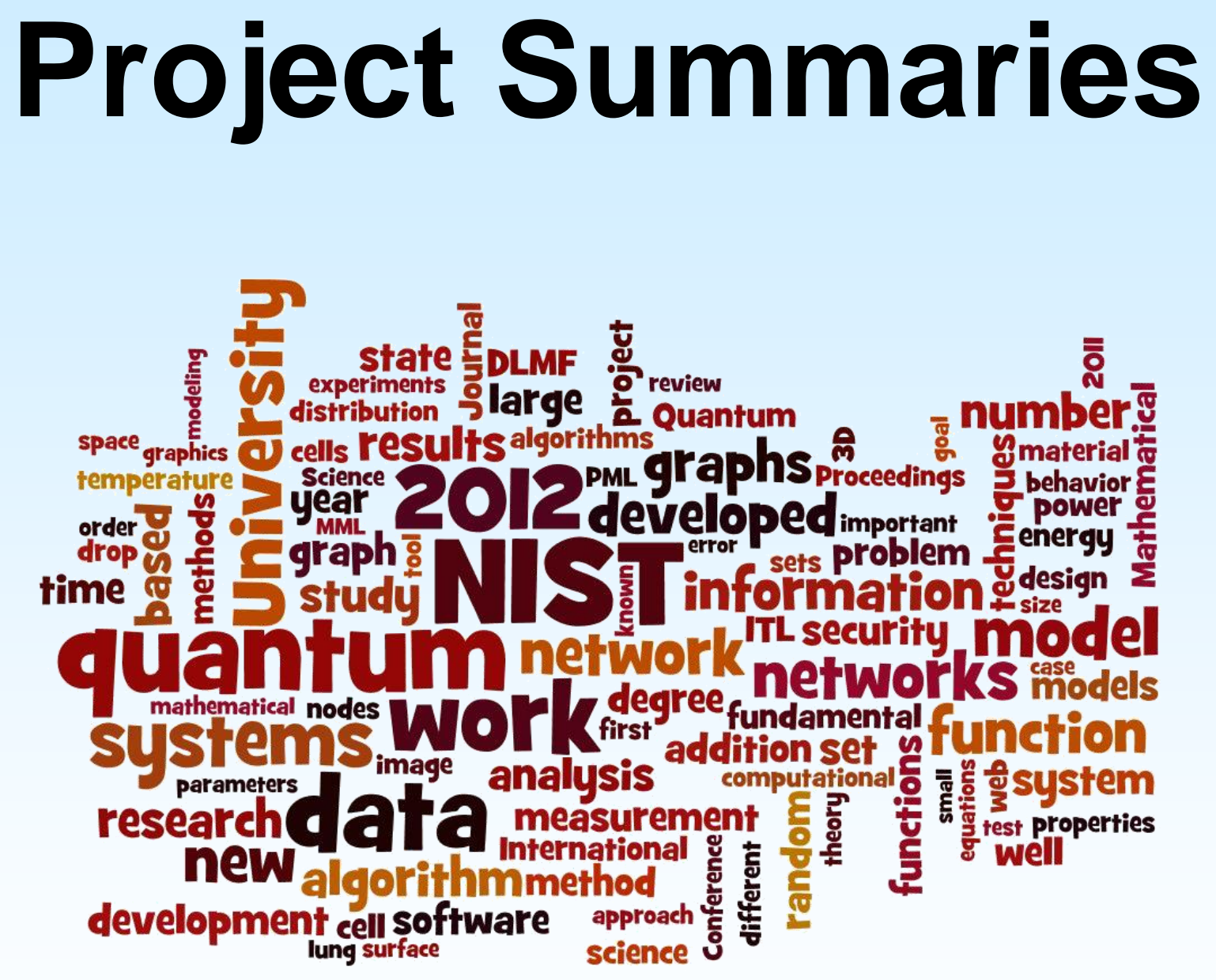


\section{Mathematics of Metrology}

Mathematics plays an important role in the science of metrology. Mathematical models are needed to understand how to design effective measurement systems, and to analyze the results they produce. Mathematical techniques are used to develop and analyze idealized models of physical phenomena to be measured, and mathematical algorithms are necessary to find optimal system parameters. Finally, mathematical and statistical techniques are needed to transform the resulting data into useful information. Our goal is to develop fundamental mathematical methods and analytical tools necessary for NIST to continue as a world-class metrology institute, and to apply them to critical measurement science applications.

\section{Continuation Backward in Time in 2D Nonlinear Parabolic Equations}

Alfred Carasso

See feature article, page 22

\section{Micromagnetic Investigation of Periodic Cross-Tie/Vortex Wall Geometry}

Michael Donahue

See feature article page 19.

\section{Metrics for Manipulation and Enhancement of Forensic Images}

Andrew Dienstfrey

Alfred Carasso

Mary Theofanos (NIST SCO)

Peter Bajcsy (ITL SSD)

Haiying Guan (ITL IAD)

David Witzke (Foray Technologies)

Matt Schwarz (Schwarz Forensic Enterprises)

See feature article, page 25.

\section{A Stochastic Theory of Fatigue for Aging Structures}

Jeffrey T. Fong

N. Alan Heckert (ITL SED)

James J. Filliben (ITL SED)

Richard A. Fields (NIST MML)

Stephen W. Freiman (NIST MML)

Pedro V. Marcal (MPACT Corp.)

Y. J. (Bill) Chao (University of South Carolina)
Poh-San Lam (Savannah River National Laboratory) Owen F. Hedden (ASME Codes \& Standards)

See feature article, page 34.

[Contributes to Virtual Measurements crosscut.]

\section{Molecular Movies: Imaging Femtosecond Motion during Electrochemical Transitions}

Bradley Alpert

Joel Ullom et al. (NIST PML)

Chris Cromer et al. (NIST PML)

Ralph Jimenez et al. (NIST PML/JILA)

Henry Kapteyn et al. (University of Colorado/JILA)

Vital to the development of next-generation nanomaterials, including photovoltaics and industrial catalysts, is an understanding gained through measurement of electron release, transport, and transfer in engineered nanostructures. This project, supported for the last two years by a NIST Innovations in Measurement Science award, proposes a revolutionary, tabletop x-ray imaging system to capture the motion of electrons, atoms, and molecules on femtosecond time scales and with picometer spatial resolution.

The combination of table-top x-ray lasers, a dramatic recent breakthrough developed at JILA, with transition-edge sensor (TES) microcalorimeter spectroscopy, intensively developed and refined in the NIST Quantum Electronics and Photonics Division, promises to enable these new measurement capabilities. The integration of these components, accompanied by significant increase in detector array sizes, to achieve large increases in temporal and spatial resolution while maintaining extraordinary TES energy resolution, requires new data modeling and processing techniques. These techniques will overcome current limitations by

- Resolving temporal overlap in photon detection while achieving energy resolution of temporally isolated arrivals, 

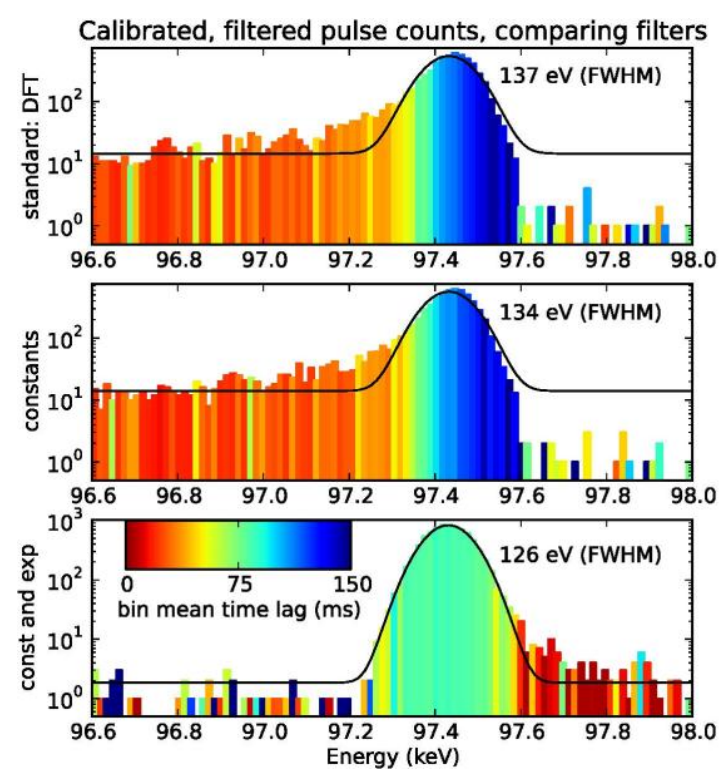

Figure 24. $97 \mathrm{keV}$ line histogram. Photon-count histograms near the $97.431 \mathrm{keV}$ gamma-ray emission line of ${ }^{153} \mathrm{Gd}$ for an average pulse rate of $13.15 \mathrm{~Hz}$ are shown for three processing filters. The standard "Wiener" filter constructed by means of the discrete Fourier transform (DFT) on the average pulse and the power spectral density is in the top subplot. In the middle subplot is shown the result of a filter constructed from the average pulse and noise autocorrelation with the new time-domain procedure that would coincide with the standard procedure if the DFT were a true Fourier transform but which in fact obviates artifacts from the DFT's artificial periodicity. The bottom subplot shows the result of the new procedure when the filter is constrained to be orthogonal to a decaying exponential tail $(\tau=6 \mathrm{~ms})$, reducing sensitivity to prior pulses. (For all three plots, the filters are orthogonal to constants, to eliminate baseline sensitivity.) Color denotes the pulse arrival time lag since the previous pulse, averaged over the histogram bin, and illustrates that filtering errors, concentrated in heavily piled-up pulses, are nearly eliminated by the filter orthogonal to exponentials.

- Improving efficiency in elimination of lowfrequency background noise, and

- Extending multiplexing and reducing cross talk in extracting the signals from below 1 degree Kelvin to room temperatures.

Wiener filtering, long used among astronomers for estimating amplitudes of pulses of known shape contaminated with noise of known frequency content, is suitable for measuring isolated pulses. Novel processing approaches are being developed and characterized that rely on this knowledge but are suitable for overlapping pulses.

This year progress occurred in the following areas.

1. Alpert's proposed method for computing filters in the time domain, rather than the frequency domain, allowing for orthogonality to prior pulse tails, was evaluated on gamma-ray data. In addition to an initial successful test at low rate pulses [1], a subsequent experiment demonstrated its better preservation of excellent energy resolution at high pulse rates than that possible under the standard method [2].

2. A filtering method recently proposed by other researchers, which is intended for inline processing and trades some resolution for simplicity and ability to handle high pulse rates, was analyzed [3].

3. An efficient procedure was developed for noise whitening, in which a photon detector's observed noise power spectrum is approximated by a rational function and a time-domain infinite impulse response filter is constructed. This procedure is expected to enable a nonlinear least squares procedure for processing piled-up pulses to be performed efficiently.

Attention is currently focused on recovering photon energies when the corresponding pulses are so closely spaced that the detector response is significantly nonlinear. In addition, the new experimental apparatus is now close to fully functional and we expect further sources of uncertainty will arise and need characterization.

[1] B. Alpert, J. Fowler, D. Bennett, R. Doriese, J. Ullom, A. Hoover, N. Hoteling, and M. Rabin, Improved High Rate Pulse Processing Algorithms for Microcalorimeters, 14th International Workshop on Low Temperature Detectors, August 2, 2011.

[2] B. K. Alpert, R. D. Horansky, D. A. Bennett, W. B. Doriese, J. W. Fowler, A. S. Hoover, M. W. Rabin, and J. N. Ullom, Filters for High Rate Pulse Processing, in review.

[3] B. K. Alpert, W. B. Doriese, J. W. Fowler, J. N. Ullom, Predicted Energy Resolution of a Running-Sum Algorithm for Microcalorimeters, Journal of Low Temperature Physics 167:5-6 (2012), 582-587.

[4] W. B. Doriese, B. K. Alpert, J. W. Fowler, G. C. Hilton, A. S. Hojem, K. D. Irwin, C. D. Reintsema, D. R. Schmidt, G. M. Stiehl, D. S. Swetz, J. N. Ullom, L. R. Vale, Optimization of the TES-Bias Circuit for Multiplexed Microcalorimeter Array, Journal of Low Temperature Physics 167:5-6 (2012), 595-601.

[5] D. A. Bennett, R. D. Horansky, D. R. Schmidt, A. S. Hoover, R. Winkler, B. K. Alpert, J. A. Beall, W. B. Doriese, J. W. Fowler, C. P. Fitzgerald, G. C. Hilton, K. D. Irwin, V. Kotsubo, J. A. B. Mates, G. C. O’Neil, M. W. Rabin, C. D. Reintsema, F. J. Schima, D. S. Swetz, L. R. Vale, J. N. Ullom, A High Resolution Gamma-ray Spectrometer based on Superconducting Microcalorimeters, Review of Scientific Instruments 83:9 (2012), 093113. 


\section{Virtual Measurements in Quantum Chemistry}

\author{
Raghu Kacker \\ Karl Irikura (NIST MML) \\ Russell Johnson III (NIST MML) \\ Ruth Jacobsen (NIST MML)
}

By a virtual measurement we mean a prediction along with its associated uncertainty for the value of a measurand determined from a computational model as an alternative to a physical measurement. Computational models are improving and are being increasingly used in science and engineering. An important application is quantum chemistry, where carefully determined uncertainties associated with computationally determined values have not been reported. As the technology improves, the need and importance of reliable uncertainties is being recognized. This project is focused on developing and applying methods for quantifying the uncertainty associated with a virtual measurement in quantum chemistry. The benefits accrue to research and development of chemical processes, materials development, and drug discovery.

Predictions from computational quantum chemistry models seldom agree with the corresponding highquality physical measurements. The differences are not random but systematic. Therefore, a common practice is to apply an empirical scaling factor to computational predictions to bring them closer to the true quantity values. The empirical scaling factor carries uncertainty. We have developed a methodology to quantify the uncertainty associated with a scaling factor. This approach is based on the Guide to the Expression of Uncertainty in Measurement, which is an international standard. The uncertainties for scaling factors lead to the corresponding uncertainties for virtual predictions.

We had reported in the Journal of Physical Chemistry (2005) scaling factors and uncertainties for vibrational harmonic frequencies. We had reported in the Journal of Chemical Theory and Computation (2010) scaling factors and uncertainties for vibrational anharmonic frequencies. Anharmonic frequencies are calculated from higher order models and require considerably more computational effort. Therefore anharmonic calculations are significantly more expensive than harmonic calculations. In 2011 and 2012 we determined that without empirical scaling, anharmonic frequencies are clearly more accurate than harmonic frequencies, as expected. However, the scaled frequencies for both harmonic and anharmonic calculations are all of comparable accuracy. The much greater cost of anharmonic calculations does not provide a benefit for the electronic structure methods. Our paper entitled "Anharmonic vibrational frequency calculations are not worthwhile for small basis sets" has been accepted for publication in the Journal of Chemical Theory and Computation [1]. We continue to address uncertainties with the outputs of computational quantum chemistry models.

[1] R. L. Jacobsen, R. D. Johnson III, K. K. Irikura and R. Kacker, Anharmonic Vibrational Frequency Calculations are not Worthwhile for Small Basis Sets, Journal of Chemical Theory and Computation, to appear.

[Contributes to Virtual Measurements crosscut.]

\section{Bubble Motion and Size Variation during Thermal Migration with Phase Change}

\author{
Asha Nurse \\ Geoffrey McFadden \\ Sam Coriell (NIST MML)
}

If a temperature gradient is applied to a fluid containing liquid drops or gas bubbles, the drops can migrate to the direction of higher temperature, even overcoming the buoyancy forces on the drops. This migration is due to the Marangoni forces that arise due to stresses associated with the temperature variation of the surface energy at the fluid-fluid interface. Generally the surface energy tends to decrease with temperature, and the resulting tangential flow along the interface is from the hotter end of the bubble to the cooler end, moving the bubble into the region of higher temperatures. Marangoni effects arise in many material processing techniques and can have a significant effect on the quality of the resulting material.

Steve Hardy, a NIST material scientist, conducted a series of experiments on the motion of an air bubble in silicone oil under carefully controlled thermal conditions [1]. He measured the vertical position and diameter of the air bubble for a specific temperature gradient and observed oscillations of both the bubble position and the radius when the system is heated from below. Hardy attributed the oscillations to the diffusion of air into or out of the bubble from the surrounding silicone oil as the bubble moves into regions of higher or lower temperature. At the turning points of the motion where the velocity vanishes, Hardy used the theoretical analysis for the thermal migration of an immiscible bubble developed by Young, Goldstein, and Block [2], who obtained an expression for the velocity of a migrating bubble as a function of the temperature gradient, gravitational acceleration, and material properties of the system. In this way Hardy was able to measure the variation of the surface energy with temperature. The model of Young et al. is not able to predict the oscillations in position and radius of the bubble that Hardy observed, however. 


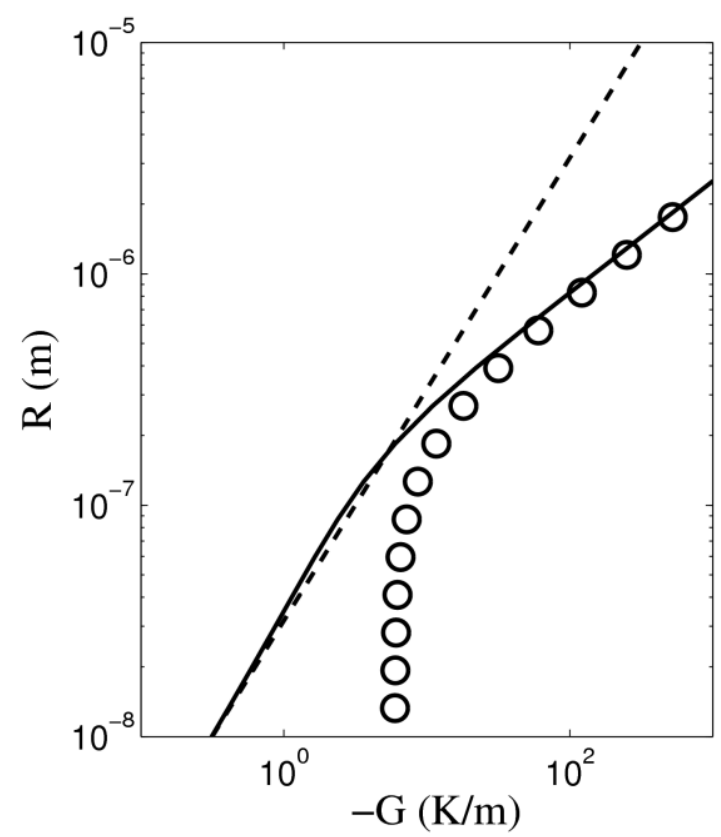

Figure 25. The steady state radius, $R$, versus temperature gradient, $G$, for a stationary bubble of steam in water heated from below at $373.15 \mathrm{~K}$. The solid curve is our exact solution; open circles show results obtained by "turning off" the Marangoni effects, and the dashed curve shows the immiscible bubble results of Young et al.

Generally motivated by Hardy's observations, we extend [3] the analysis of Young et al. by considering a drop that is the vapor phase of the surrounding unbounded liquid and vice versa. The drop radius and position are both allowed to vary in time as flow normal to the interface occurs due to phase change. We assume the flow is governed by the incompressible Stokes equations and is coupled to a quasi-static thermal field. The flow equations are solved with associated boundary conditions that include the release of latent heat upon a change of phase at the surface, and also permit flow through the surface of the bubble, in contrast to the case of the thermal migration of an immiscible drop.

For a spherical drop, an explicit solution gives a pair of coupled ordinary differential equations that govern the rate of change of the drop radius and position. Our expression for the drop velocity contains terms proportional to the temperature gradient and the gravitational acceleration, just as in the analogous expression obtained by Young et al. However, our expression contains a number of additional materials parameters, resulting in a more complicated dependence on the drop radius. The steady state solutions of these equations generally describe a balance between buoyancy and thermal effects and is illustrated using material properties corresponding to a water-steam system in Figure 25. For smaller drops, the results are similar to those for the immiscible case treated by Young et al [2], while for larger drops, the Marangoni effect can be overwhelmed by the effects of latent heat release.

We have analyzed the linear stability of the steadystate solutions of the governing equations and found conditions that lead to solutions that oscillate in position and radius as were observed experimentally by Hardy. We note that a drop or bubble with an immiscible interface cannot undergo purely radial oscillations if the flow is incompressible, since there is no local mass flux through the interface. Analogous to Hardy's diffusion problem, the cycle of oscillation here is that a downward displacement into a region of higher temperature produces an increase in bubble radius due to evaporation; the larger bubble then rises due to increased buoyancy. Overshooting its original position, it then rises to regions of colder temperature, where it shrinks due to condensation and falls due to reduced buoyancy to repeat the cycle. We have also performed a weakly-nonlinear analysis of the oscillatory modes, and compared the result with a numerical solution of the nonlinear equations. Future work will extend this single-component model to include the effects of gaseous diffusion, which should allow more accurate comparisons with the experimental results of Hardy.

[1] S. C. Hardy. The Motion of Bubbles in a Vertical Temperature Gradient, Journal of Colloid and Interface Science 69 (1979), 157-162.

[2] N. G. Young, J. S. Goldstein, and M. J. Block, The Motion of Bubbles in a Vertical Temperature Gradient, Journal of Fluid Mechanics 6 (1959), 350-356.

[3] A. K. Nurse, G. B. McFadden, and S. R. Coriell, Bubble Motion and Size Variation during Thermal Migration with Phase Change, in review.

\section{On the Stability of Rotating Drops}

\author{
Asha Nurse \\ Geoffrey McFadden \\ Sam Coriell (NIST MML)
}

Analyses of the dynamics of a rotating liquid drop held together by surface tension were initiated by Plateau (1863). Generally, an effectively weightless liquid drop is immersed in an immiscible liquid and then spun at a controllable rate. Assuming rigid body motion and taking into account solely axisymmetric drop configurations, the drops evolve from spherical configurations at zero rotation rate through a family of axisymmetric shapes that progressively flatten at the poles while developing an equatorial bulge. At large enough rotation rates, Plateau observed transient toroidal configurations that tended to break up into smaller drops.

Recently, there has been interest in the stability of toroidal shapes. Experiments on neonatal fibroblast 


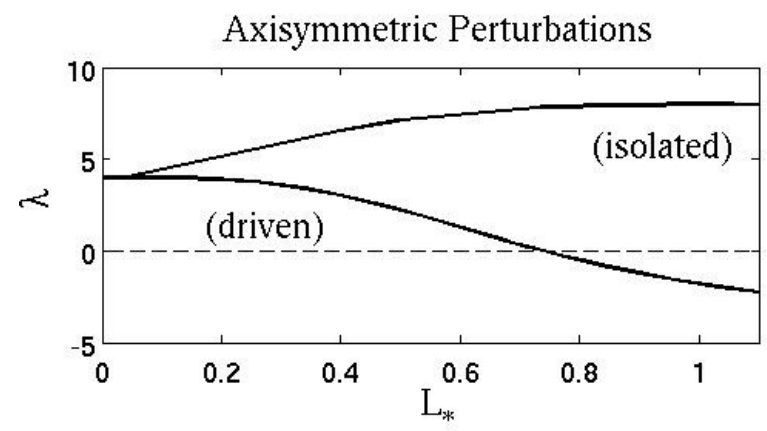

Figure 26. Linear stability of rotating spheroidal drops. The least stable values of $\lambda$ for axisymmetric disturbances $(n=0)$ are shown for driven and isolated drops as a function of the normalized angular momentum $L_{*}$

cells that have self-assembled into a toroidal cluster about the base of a conical pillar, have shown that the toroidal cluster will actively do work to climb the pillar to become a sphere or will remain at the base of the pillar and break up to form smaller clusters [1]. Subsequent theoretical work on this self-assembled system points to the minimization of the surface energy as a key factor in the configurational driving force for the motion of the cluster [2]. As for the thinner toroidal clusters that do not climb the conical pillar, the development of localized deformations along its circumference is viewed to as a result of the unstable growth of surface fluctuations. These works have revived interest in the stability of a rotating drop held together by surface tension, which we consider here.

Theoretical analysis of the spherical liquid drop and its resulting axisymmetric shapes has been done by Brown and Scriven [3], who performed finite element computations of the deformed shapes, and by Chandrasekhar [4], who included fluid flow inside the drop and used a method of moments. We employ a Lagrangian energy functional that represents the difference between a drop's potential energy, due to capillary forces, and the kinetic energy of rotation, subject to a volume constraint. The stationary points of the energy functional are used to determine the equilibrium shapes of spheroidal and toroidal drops. We obtain numerical solutions for these shapes in terms of an angle arc length formulation.

For a drop undergoing rigid body rotation with a fluid of a different density, the application of an external torque may be necessary to maintain a given rotation rate. This is referred to as a driven drop. Alternatively, if there is no applied external torque, the drop rotates with constant angular momentum and is termed an isolated drop. The energy functional for the drop is a function of the drop's shape and angular momentum. The stability of either case can be described in terms of the second variation of their energy functionals. For axisymmetric perturbations stability results for driven and isolated drops differ, as shown in Figure 26, with the isolated drop being more stable than the driven drop. Here $\lambda$ is an eigenvalue determining the linear stability of the rotating drop, with negative values of $\lambda$ corresponding to instabilities that are saddle points in the energy profile. In our work we have derived expressions for the second variation of the energy of both isolated and driven drops, and obtained numerical results for the stability of rotating spheroidal and toroidal configurations. In particular we find that toroidal drops with large major radii are subject to a wide class of non-axisymmetric perturbations, as suggested by Plateau's original experiments. Future work will include the modeling of the interaction of a toroidal drop with an underlying substrate, which may allow more accurate simulations of the observed instabilities in the fibroblast cellular clusters.

[1] J. Youssef, A. K. Nurse, L. B. Freund and J. R. Morgan, Quantification of the forces driving self-assembly of three-dimensional microtissues, Proceedings of the National Academy of Science 108 (2011) 6993-6998.

[2] A.K. Nurse, L. B. Freund and J. Youssef, A Model of Force Generation in a Three Dimensional Toroidal Cluster of Cells, Journal of Applied Mechanics 79 (2012) 1013.

[3] R.A. Brown and L.E. Scriven, The shape and stability of rotating liquid drops, Proceedings of the Royal Society of London A 371 (1980), 331-357.

[4] S. Chandrasekhar, The stability of a rotating liquid drop, Proceedings of the Royal Society of London A $\mathbf{2 8 6}$ (1965), 1-26.

\section{Moving Grain Boundary Groove Under Stress}

\section{Geoffrey McFadden \\ William Boettinger (NIST MML) \\ Robert Sekerka (Carnegie Mellon University)}

Grain boundaries are the interfaces that separate individual grains of a crystalline material that have different orientations of the crystal axes in each grain. Grain growth occurs by grain boundary motion, which is subject to energetic driving forces from effects such as surface tension, which tends to minimize net surface energy, and elastic forces, which tend to minimize strain energy. In thin films of polycrystalline material, the presence of lateral compressive or tensile stresses can cause migration of a grain boundary with an associated deformation of the nearby solid-vapor interface at the film surface. An example in a tin system is shown in Figure 27. The formation of such "hillocks" is commonly observed in thin films under compression, and has been conjectured to lead to the formation of crystalline "whiskers" that can bridge gaps between electronic components and lead to electrical failure. 


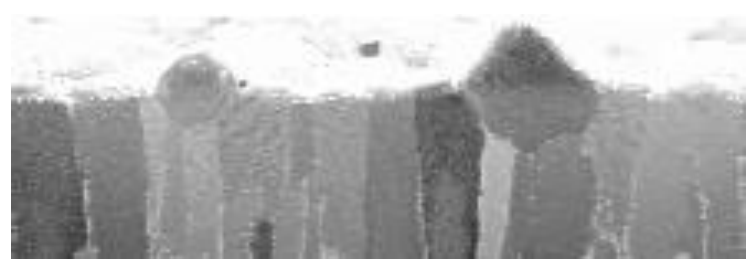

Figure 27. Scanning electron micrograph view of a cross section of a tin electrodeposit containing hillocks. There is evidence of grain boundary motion in the deposit under each hillock.

Models of grain boundary motion when the slope of the solid-vapor interface is small have been developed by Mullins and co-workers in a variety of contexts $[1,2]$. In the present work we have extended that work by improving the description of the grain boundary junction where the interfaces intersect, and by extending the model to finite slopes. In a sample with two-dimensional geometry this results in a system of nonlinear fourth-order ordinary differential equations in a coordinate system that moves with the velocity of the grain boundary. The model contains dimensionless parameters that represent the ratios of surface energies, $m$, the flux of atoms up the grain boundary due to stress, $j$, kinetic effects due to differences in chemical potentials at the groove, $c$, and the groove velocity, $a$. An example is shown in Figure 28, where the variation in the shape of the solid-vapor interface is shown for a given groove velocity, $a$, with no applied stress or kinetic effects, as the surface energy changes. A linear theory can be used when the slopes are small (small $m$ ), and the figure shows a comparison of those results with a nonlinear theory that is valid for finite slopes (larger $m$ ). The groove is moving to the right, and the elevation of surface height to the right of the groove represents the formation of a hillock in this model.

Future work may include the effects of perturbations perpendicular to the groove, which are observed as corrugated structures along the grains, and simula-

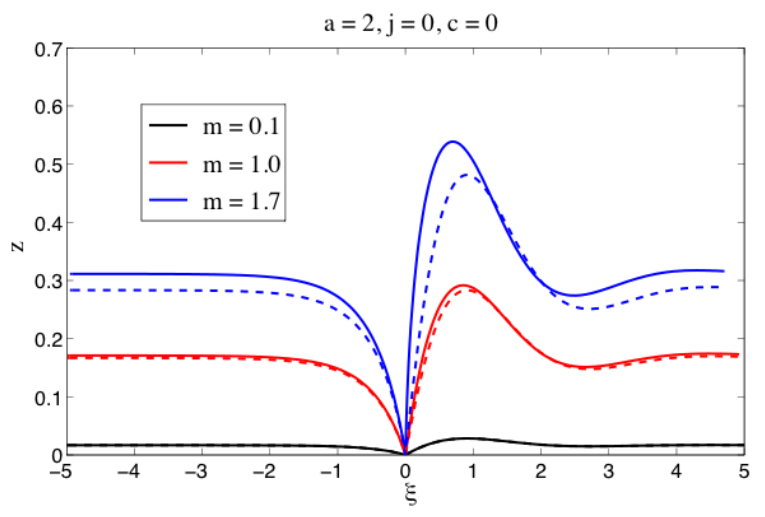

Figure 28. Groove shapes for $a=2$, with $j=c=0$. The dotted lines show the linear (small slope) solution, and the solid curves are the numerical (finite slope) solution. tions of the dynamical behavior of the groove in response to variations in stress.

[1] W. W. Mullins, The Effect of Thermal Grooving on Grain Boundary Motion, Acta Metallurgica 6 (1958), 414-427.

[2] F. Y. Genin, W. W. Mullins and P. Wynblatt, The Effect of Stress on Grain Boundary Grooving, Acta Metallurgica 41 (1993), 3541-3547.

\section{Measurement and Modeling of Plastic Flow Stress for High-Speed Machining Simulations}

\author{
Timothy Burns \\ Steven Mates (NIST MML) \\ Richard Rhorer (NIST EL) \\ Eric Whitenton (NIST EL) \\ Debasis Basak (Orbital Sciences Corporation)
}

A high-speed metal machining operation provides an interesting and industrially important setting for the study of plastic flow under extreme conditions. In the thin region where cutting takes place, it is not uncommon, in alloys of interest in manufacturing, to have shear strains on the order of $200 \%$, shear strain rates on the order of $10^{4} \mathrm{~s}^{-1}$, and heating so rapid that the local workpiece temperature increases from ambient to a significant fraction of the melting temperature in a time on the order of a tenth of a second or less. In order to obtain useful predictive computer simulations of the cutting conditions during a high-speed metal machining operation, such as the cutting forces and the temperature along the interface between the tool and the work material, a constitutive response model for the flow stress in the work material, and a model for the friction between the workpiece and the cutting surface of the tool, must be specified. Despite years of research on this subject, the current state of the art is unsatisfactory. For example, in a study that used data from cutting experiments performed at NIST in 2007 [1], it was found that the force predictions made using a state of the art finite-element software package that was developed specifically for machining simulations, were off by about $20 \%$.

In work performed this year [2], an analysis was made using some experimental measurements of the temperature along the tool-chip interface (segment A-B in Figure 30) during some high-speed machining tests [3], together with an empirically determined tool-chip interface friction model, to make an estimate of the flow stress in the workpiece material that provided the best peak temperature prediction for four different sets of cutting tests that had been performed at NIST. This stress estimate was then compared with two different 


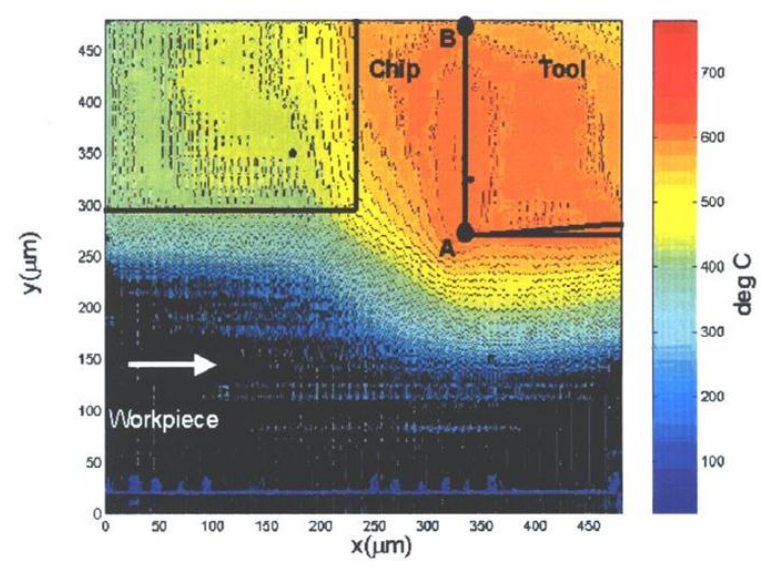

Figure 30. False color thermal image of high-speed, steady-state orthogonal machining of AISI 1045 steel; taken from [3].

constitutive models for the flow stress for the same material, which had been determined, respectively, using data that were measured in two different sets of experiments. The first set of experiments was performed on a Kolsky bar in a Dutch laboratory. In these tests, which were performed specifically for application to the study of high-speed machining, the sample material was pre-heated using a small oven, prior to loading the sample in compression [4]. In the second set of tests, which were performed on the NIST pulse-heated Kolsky bar, the sample material was pre-heated much more rapidly prior to loading in compression, and it was found that the material exhibited a significantly stiffer constitutive response, i.e., a higher shear stress at the same strain, strain rate, and temperature [5]. The results of this study are summarized in Figure 29. The stiffer response of the pulse-heated data is evident. However, it is clear that neither constitutive model for the flow stress that was determined using Kolsky bar experimental data set does an overall good job of matching the stress that was estimated independently.

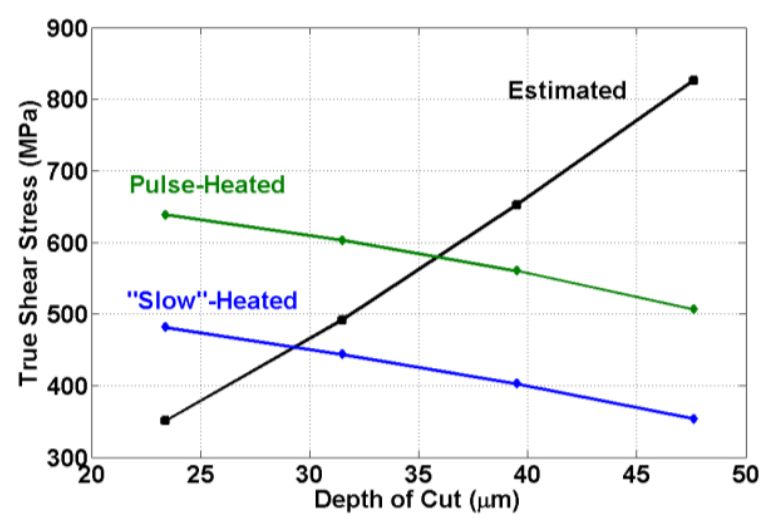

Figure 29. Comparison of estimated shear flow stress during four different high-speed cutting tests (black) with shear stress predicted from constitutive models based on "slow"-heated (blue) and NIST pulse-heated Kolsky bar data (green).
In work in progress, this method of estimating the stress using an interface friction model together with in-situ temperature measurements during high-speed cutting tests, is being extended using a more sophisticated model for the friction between the tool and the work material.

[1] R. Ivester, E. Whitenton, J. Heigel, T. Marusich and C. Arthur, Measuring Chip Segmentation by High-speed Microvideography, and Comparison to Finite-element Modeling Simulations, in Proceedings of the 10th CIRP International Workshop on Modeling of Machining Operations, Calabria, Italy, August 27-28, 2007.

[2] T. J. Burns, S. P. Mates, R. L. Rhorer, E. P. Whitenton and D. Basak, Transient Convection-diffusion Modelling of Peak Temperature in Orthogonal Cutting, in Proceedings of the 23rd International Congress of Theoretical and Applied Mechanics (ICTAM2012), Beijing, China, August 19-24, 2012.

[3] M. A. Davies, H. Yoon, T. L. Schmitz, T. J. Burns and M. D. Kennedy, Calibrated Thermal Microscopy of the Tool-chip Interface in Machining, Machining Science \& Technology 7 (2003), 166-190.

[4] S. P. F. C. Jaspers and J. H. Dautzenberg, 2002, Material Behaviour in Conditions Similar to Metal Cutting: Flow Stress in the Primary Shear Zone, Journal of Materials Processing Technology 122 (2002), 322-330.

[5] T. J. Burns, S. P. Mates, R. L. Rhorer, E. P. Whitenton and D. Basak, Modelling the Peak Cutting Temperature during High-speed Machining of AISI 1045 Steel, Engineering Transactions 60:2 (2012), 118-124.

\section{Selecting an Appropriate Statistical Distribution for a Class of Engineering Material Property Data}

Jeffrey T. Fong

N. Alan Heckert (ITL SED)

James J. Filliben (ITL SED)

Stephen W. Freiman (NIST MML)

Pedro V. Marcal (MPACT Corporation)

One of the most hotly-debated questions in engineering design and failure analysis is how best to fit a set of uniaxial ultimate strength data. In an ASTM recommended standard practice for advanced ceramic materials [1], it is assumed that a two-parameter Weibull distribution with a zero location parameter will "best" fit the data. This leads to a physically unrealistic and highly conservative assumption that among all possible samples of an engineering material, one will fail a uniaxial tensile strength test at zero load. Also unduly conservative will be the so-called A- and Bbasis design allowables for failure strength at $95 \%$ confidence when the location parameter is assumed to be at the origin. 
In this work, we take a brute-force and somewhat exhaustive approach by developing a screening tool to answer the question how "best" a given set of univariate strength data will fit a distribution without assuming a zero location parameter. We select two methods of parameter estimation and apply four criteria of goodness-of-fit to more than 60 commonly-known distributions to see how they rank among themselves according to each of the eight combinations of parameter-estimation method plus goodness-of-fit criterion. We then develop a composite index for the eight individual rankings and order all 60+ distributions in a list for selecting the most "appropriate" distribution for the given data set.

The two parameter estimation methods are:

1. Maximum Likelihood [2].

2. Probability Plot Correlation Coefficient [3].

The four goodness-of-fit criteria are:

a. Kolmogorov-Smirnov [4].

b. Anderson-Darling [5, 6].

c. Akaike-Burnham-Anderson [7, 8].

d. Probability Plot Correlation Coefficient [3].

Using public-domain statistical analysis software named DATAPLOT [9], we develop a generic screening tool, and demonstrate its feasibility by applying to three sets of data, one for a glassy material and two for steels [10].

[1] ASTM, Standard Practice for Reporting Uniaxial Strength Data and Estimating Weibull Distribution Parameters for Advanced Ceramics, ASTM Designation: C 1239-07, Committee C28 on Advanced Ceramics, reapproved 2008, West Conshohocken, PA, 19428-2959.

[2] J. Aldrich, R. A. Fisher and the Making of Maximum Likelihood 1912-1922, Statistical Science 12 (3) (1997), 162-176.

[3] J. J. Filliben, The Probability Plot Correlation Coefficient Test for Normality, Technometrics 17 (1) (1975), 111-117.

[4] F. J. Massey Jr., The Kolmogorov-Smirnov Test for Goodness of Fit, Journal of the American Statistical Association 46 (1951), 68-78.

[5] T. W. Anderson and D. A. Darling, Asymptotic Theory of Certain 'Goodness of Fit' Criteria based on Stochastic Processes, Annals of Mathematical Statistics 23 (1952), 193-212.

[6] T.W. Anderson and D. A. Darling, A Test of Goodness of Fit, Journal of the American Statistical Association 49 (1954), 765-769.

[7] H. Akaike, A New Look at the Statistical Model Identification, IEEE Transactions on Automatic Control 19 (6) (1974), 716-723.

[8] K. P. Burnham and D. R. Anderson, Multimodel Inference: Understanding AIC and BIC in Model Selection," Sociological Methods and Research 33 (2004), 261-304.
[9] J. J. Filliben and N. A. Heckert, Dataplot: A Statistical Data Analysis Software System ${ }^{12}$, National Institute of Standards and Technology, Gaithersburg, MD 20899, 2002.

[10] J. T. Fong, N. A. Heckert, J. J. Filliben, P. V. Marcal, and S. W. Freiman, A Multi-Distribution and MultiGoodness-of-Fit-Criterion Method of Finding the Most Probable Underlying Distribution for a Given Set of Univariate Data, in preparation.

\section{A Bayesian Nondestructive Evaluation Approach to Estimating Remaining Life of Aging Structures}

\author{
Jeffrey T. Fong \\ N. Alan Heckert (ITL SED) \\ James J. Filliben (ITL SED) \\ William F. Guthrie (ITL SED)
}

The formulation of a stochastic multi-crack-growth (MCG) -based theory of fatigue for aging structures [1] paved the way for us to use the powerful Bayesian statistical method for non-destructive evaluation (NDE) data analysis. This method allows us to use prior knowledge of the likely distributions of the crack length and crack-growth-rate data to iterate for the best estimation and to validate the model to make sure it provides a reasonable description of the variation in the observed data.

With the Bayesian analysis software WinBUGS [2] as a simulation platform, we used a Bayesian statistical model fit with a Markov-Chain-Monte-Carlo (MCMC) algorithm [3-7] to implement the four governing equations as reported in [1]. Since most of the NDE data on inspection of commercial pressure vessels and piping are proprietary, we used a set of synthetic data of the crack growth of five cracks as input to an example of the MCG-based theory.

[1] J. T. Fong, N. A. Heckert, and J. J. Filliben, A Stochastic and Local Theory of Fatigue for Aging Pressure Vessels \& Piping, in From Failure to Better Design, Manufacture and Construction, (S. T. Tu, Z. D. Wang, and G. C. Sih, eds.), Proceedings of the $3^{\text {rd }}$ International Symposium on Structural Integrity, Oct. 31 - Nov. 4, 2012, Shandong University, Jinan, China, East China University of Science and Technology Press, Shanghai, China, 2012, 19-31.

[2] D. J. Lunn, A. Thomas, N. Best, and D. Spiegelhalter, WinBUGS - A Bayesian Modeling Framework: Concepts, Structure, and Extensibility, Statistics and Computing 10 (2000), 325-337.

[3] A. Gelman, J. B. Carlin, H. S. Stern, and D. B. Rubin, Bayesian Data Analysis, Chapman \& Hall, London 1995.

\footnotetext{
${ }^{12}$ http://www.itl.nist.gov/div898/software/dataplot.html
} 
[4] D. Berry, Statistics: A Bayesian Perspective, Wadsworth Publishing Co., Belmont, CA, 1996.

[5] P. M. Lee, Bayesian Statistics: An Introduction, $2^{\text {nd }}$ edition, Arnold Publishing, London, 1997.

[6] O. Haggstrom, Finite Markov Chains and Algorithmic Applications, Cambridge University Press, 2002.

[7] G. Casella, and E. I. George, Explaining the Gibbs Sampler, American Statistician 46 (1992), 167-174.

\section{Simulation of Bioregulatory Networks Involved in Cell Cycle Control}

\section{Geoffrey B. McFadden}

Mirit Aladjem (National Institutes of Health)

S. Kim (National Institutes of Health)

Kurt Kohn (National Institutes of Health)

A. Luna (NIH and Boston University)

G. McFadden is a co-advisor to Sohyoung Kim, an NIH post-doctoral fellow, and Augustin Luna, a graduate student from Boston University, who are both doing research at the National Institutes of Health (NIH); their co-advisors are NIH researchers M. Aladjem and $\mathrm{K}$. Kohn. The team is developing models of bioregulatory networks that are involved in cell cycle control. The models consist of systems of nonlinear ordinary differential equations that typically exhibit switching behavior, limit cycles, and other types of bifurcations. Proper cell growth depends on a network of interacting molecules that monitors cellular metabolism and environmental signals. This network ensures that cells halt their growth in response to unfavorable conditions such as the absence of sufficient nutrients or the presence of potentially damaging agents. When cells escape these controls, the results are developmental abnormalities, genomic instability, and cancer.

Current work by Sohyoung Kim focuses on a protein known as p53, a tumor suppressor that causes cell cycle arrest or programmed cell death in response to stress signals such as DNA damage. Regulating the appropriate levels of p53 is essential for cell survival. Recent modeling work [1] has suggested experimental investigations of the role played by another regulatory protein, Mdmx, in the cell cycle that are currently being performed by Dr. Kim at the Laboratory for Molecular Pharmacology at the NIH National Cancer Institute. She has developed a specialized cell line that is designed to allow the measurement of p53 levels in a single cell by tagging individual species in the cell with green florescent proteins whose luminous intensity varies with concentration (see Figure 31 ). In this way the model predictions of the role of Mdmx can be tested experimentally [2]. Future work will include the

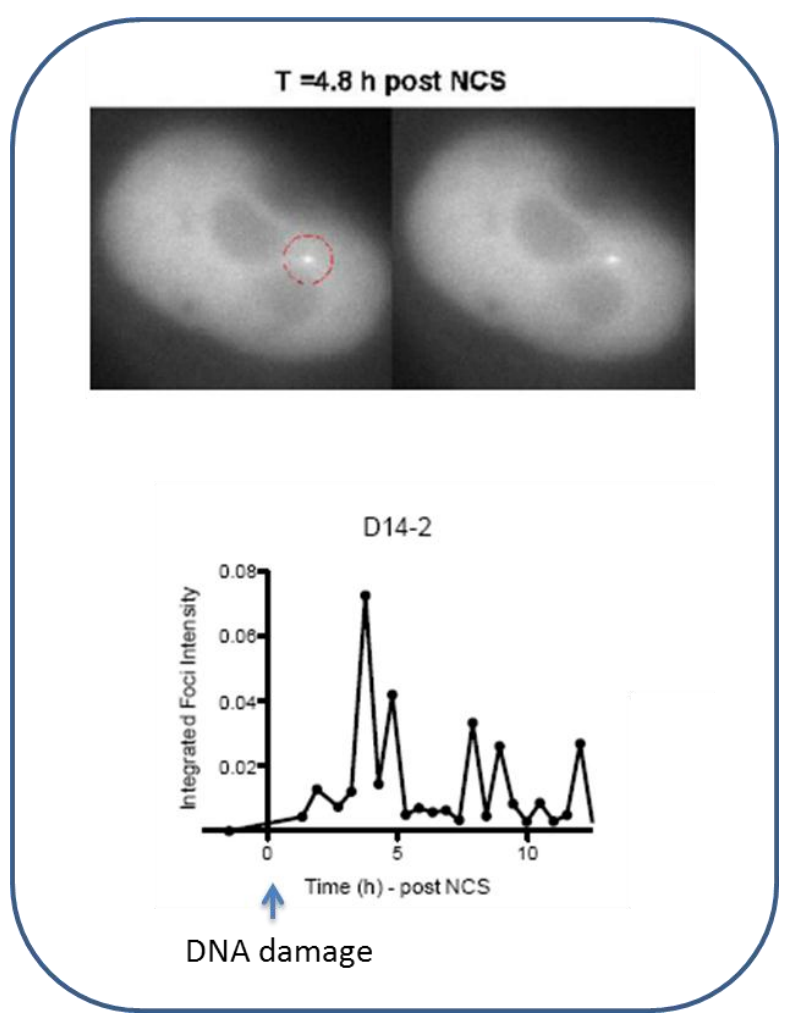

Figure 31. Images of a single cell exhibiting cyclic response to DNA damage (introduction of 'NCS' agent). The measured intensity at a foci (red highlighted circle) is a measure of the local p53 concentration.

development of refined models to better account for the newest experimental results.

Recent work by Augustin Luna includes the development of models for the role played by a protein, SIRT1, in regulating cellular response to DNA damage. This work is modeling the effects of SIRT1 on the mammalian circadian clock cycle. It is known that the

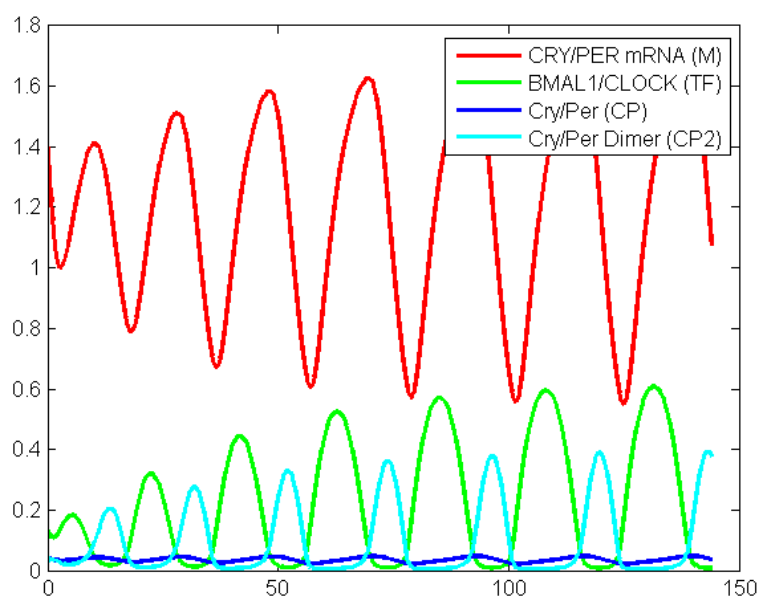

Figure 32. Numerical simulation of the dynamics of a model for the interaction between the DNA damage response and the circadian cycle in cells. The concentration of the model's species are shown as a function of time (hours). 
time of day that cancer treatments are administered affects drug efficacy, and circadian rhythms are also known to affect DNA damage response. SIRT1 is known to play a role in the DNA damage sensor that triggers the cellular response to genotoxic stress. In this work existing models for the circadian clock are being modified to include the specific effects of SIRT1 on the interaction between the DNA damage response and the circadian cycle in cells; a numerical example is shown in Figure 32.

A poster describing this work was presented at a recent international conference on systems biology [3]. This work is currently being written up as part of Dr. Luna's Ph.D. thesis, which will be defended in 2013.

[1] S. Kim, M. I. Aladjem, G. B. McFadden, and K. W. Kohn, Predicted Functions of MdmX in Fine-tuning the Response of p53 to DNA Damage, PLoS Computational Biology 6 (2010), e1000665 (18 pages).

[2] S. Kim, M. I. Aladjem, G. B. McFadden, and K. W. Kohn, Testing in silico Predictions of p53 Dynamics by Direct Measurements of Transcription Kinetics, NIH Research Festival, Bethesda, MD, October 9-12, 2012.

[3] A. Luna, G. McFadden, M. Aladjem, and K. Kohn, A Computational Mammalian Circadian Clock Model with Connections to the DNA Damage Response, $20^{\text {th }}$ Annual International Conference on Intelligent Systems for Molecular Biology, Long Beach, CA, July 15-17, 2012.

\section{Mathematical Optimization of Procedures for Cryoprotectant Equilibration}

\author{
Anthony Kearsley \\ Daniel Anderson (George Mason University) \\ James Benson (Northern Illinois University) \\ Adam Higgins (Oregon State University)
}

Cryopreservation procedures permit cells to be stored indefinitely using extremely cold temperatures to suspend all metabolic activities and therefore preserve the cell and cell function. Loosely speaking, there are two main types of cryopreservation procedures: equilibrium (conventional slow freezing) and non-equilibrium or ultra-rapid freezing (vitrification). Both procedures employ the use of cryoprotectants, chemicals behaving like antifreeze. Cryoprotectants prevent cellular damage during the freezing process. After proper freezing cells should be able to be stored for an indefinite amount of time. This can be done, for example, by immersing them in liquid nitrogen, an extremely cold fluid with an approximate temperature of -196 C. Cryoprotectants must be removed later during the thawing process and slowly the water balance in the cell can be restored and normal activity in the cell should return.
Procedures exist for the freezing of single cells, tissue comprised of many cells and entire organs. Size, amount of cytoplasm (or fluid) structural complexity change the freezing procedures employed. For example, cells with less cytoplasm, like sperm cells, are generally considered to be less difficult to freeze than those cells with more cytoplasm, like eggs. Slow freezing or equilibration has been successfully employed to freeze and store a wide range cells but research is ongoing to optimize the cryobiology of cells.

Recently we studied the use of optimization techniques to determine or improve the equilibration of cryoprotectant. We considered the case of cells and tissues with high concentrations of permeating chemicals known as cryoprotective agents (CPAs). Despite their protective properties, CPAs can cause damage as a result of osmotically-driven cell volume changes, as well as chemical toxicity. In this study, data was used to determine a toxicity cost function, a quantity that represents the cumulative damage caused by toxicity. We then used this cost function to define and numerically solve the optimal control problem for CPA equilibration, using human oocytes as representative cell type with high clinical relevance. The resulting toxicity-optimal procedures are predicted to yield significantly less toxicity than conventional procedures. Results showed that toxicity is minimized during CPA addition by inducing the cell to swell to its maximum tolerable volume and then loading it with CPA while in the swollen state. This counterintuitive result is considerably different from the conventional stepwise strategy, which involves exposure to successively higher CPA concentrations in order to avoid excessive shrinkage. The procedure identified is a first step in deriving procedures that significantly reduce damage due to toxicity.

[1] J. D. Benson, A. J. Kearsley, A. Z. Higgins, Mathematical Optimization Procedures for Cryoprotectant Equilibration using a Toxicity Cost Function, Cryobiology 64 (3) (2012), 144-151.

\section{Hurricane-Borne Missile Speeds}

\author{
Florian A. Potra \\ Emil Simiu (NIST EL) \\ Brad Harvey (Nuclear Regulatory Commission) \\ Kevin Quinlan (Nuclear Regulatory Commission)
}

The safety of nuclear power plants is a matter of paramount national concern. The U.S. Nuclear Regulatory Commission (NRC) contracted NIST to perform a study of hurricane-borne missiles (i.e., debris), and their possible impacts on nuclear power plants. The study resulted in a comprehensive technical report [2] intended to provide the technical basis for a new regulatory guide [3] that would provide licensees and 
applicants with guidance that the staff of the NRC considers acceptable for use in selecting the design-basis hurricane-borne missile speeds for the design of nuclear power plants. The design must prevent undue risk to the health and safety of the public in accordance with General Design Criterion 2, "Design Bases for Protection Against Natural Phenomena," and General Design Criterion 4, "Environmental and Dynamic Effects Design Bases," of Appendix A, "General Design Criteria for Nuclear Power Plants," to Title 10, Part 50, of the Code of Federal Regulations, "Domestic Licensing of Production and Utilization Facilities."

The report documents the approach to and results of calculations of hurricane-borne missile speeds that may be considered in the design of nuclear power plants. The missile spectrum, the assumptions on which the analyses are based -- which are similar to assumptions used for the development of Revision 1 to Regulatory Guide 1.76, "Design-Basis Tornado and Tornado Missiles for Nuclear Power Plants,"- and the range of wind speeds being considered, were based on consultations between the authors and NRC staff. In addition, calculated missile speeds based on assumptions on the initial missile height above ground that differ from the assumption used in Revision 1 to Regulatory Guide 1.76 are included in Appendixes. The staff initiated this study because Revision 1 to Regulatory Guide 1.76 was based on the new Enhanced Fujita scale, in which tornado design-basis wind speeds are lower than in the earlier Fujita scale. The design-basis tornado wind speeds presented in Regulatory Guide 1.76 correspond to an exceedance probability of $10^{-7}$ per year. This report covers missile speeds that, under several hypotheses, could occur in hurricanes with $10^{-7}$ per year probability of exceedance wind speeds. Software for estimating speeds and trajectories for different types of hurricane-borne missiles is available ${ }^{13}$.

A joint paper with NRC colleagues [1] discusses the findings of the report, and concludes that the study of extreme wind gusts during hurricanes showed that wind speeds from the design-basis tornado remain bounding except for locations along the United States Gulf Coast and the southern Atlantic Coast. The study of missile speeds during hurricanes also concluded that airborne missiles fly faster in a hurricane wind field as compared to a tornado wind field of the same strength. Consequently, applicants for new power plants with sites along the Gulf and Atlantic coasts where the design-basis tornado may not bound the design-basis hurricane are expected to show that their applicable structures can withstand, independently, the total design-basis tornado load and the total design-basis hurricane load as extreme environmental conditions. Additional comments are given in [4].

\footnotetext{
${ }^{13}$ http://www.itl.nist.gov/div898/winds/mathematica/nrc.htm
}

[1] B. Harvey, E. Simiu, F. A. Potra, and K. Quinlan, Design-Basis Hurricane Winds and Missiles for Nuclear Power Plants, in Transactions of the 22nd International Conference on Structural Mechanics in Reactor Technology, SMiRT-22, San Francisco, California, August 18-23, 2013, in review.

[2] E. Simiu and F. A. Potra, Technical Basis for Regulatory Guidance on Design-Basis Hurricane-Borne Missile Speeds for Nuclear Power Plants (NUREG/CR-7004), Office of Nuclear Regulatory Research, U.S. Nuclear Regulatory Commission, Washington, DC 20555-0001, November 2011, (110 pages).

[3] U.S. Nuclear Regulatory Commission (NRC), DesignBasis Hurricane and Hurricane Missiles for Nuclear Power Plants, Regulatory Guide 1.221, 2011.

[4] D.H. Yeo, F. T. Lombardo, D. K. Banerjee, E. J. Letvin, E. Simiu, M. L. Levitan and F. A. Potra, NIST Advances in Computer-aided and Computational Methods in Wind Engineering, in Proceedings of the ATC-SEI Advances in Hurricane Engineering Conference, Miami, FL, 2012 (12 pages).

\section{Multi-Hazard Engineering Design}

\author{
Florian A. Potra \\ Chiara Crosti (NIST EL) \\ Dat Duthinh (NIST EL) \\ Jay Harris (NIST EL) \\ Long T. Phan (NIST EL) \\ Emil Simiu (NIST EL)
}

There is a growing interest in the development of procedures for the design of structures exposed to multiple hazards, including strong winds. The goal is to achieve safer and more economical or "greener" designs than would be the case if the demands on structures were calculated independently for each individual hazard and an envelope of those demands were used for member sizing. A broad, multidisciplinary foundation for multi-hazard design should include structural analysis, optimization, and probabilistic structural reliability components. A paper presented at the 13th International Conference on Wind Engineering [1] discusses this interdisciplinary approach to engineering in a multihazard context and presents new procedures and results obtained by NIST researchers. Additional details are provided in [2].

The development of the current American Society of Civil Engineers (ASCE) 7 Standard provisions on structural design for regions with strong wind and seismic hazards is based on the belief that such provisions should be determined strictly by the fact that the probability of simultaneous occurrence of such hazards is negligible. Figure 33 represents schematically a water tower located on the coast in South Carolina, a state with both strong wind and hurricane hazards. A complete description of the structure can be found in [1]. 


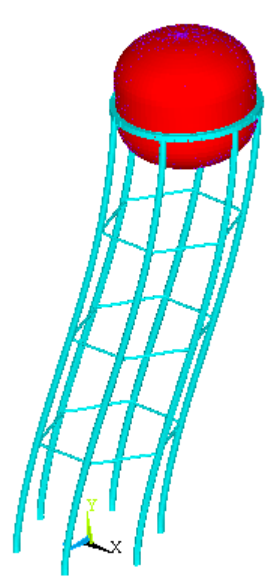

Figure 33. Schematic of water tower structure.

The calculations show that the legs of the water tower reach similar limit states under combined gravity and wind loading, and under combined gravity and earthquake loading. This means that, during its life, the legs would experience the limit states being considered with a probability that is approximately twice as large as the probability inherent in the ASCE 7-05 Standard.

A design that improves the performance of a structure under one hazard is called synergistic if it also improves the performance of the structure under a second hazard. For example, Hayes et al. [2] found that strengthening of a structure for seismic loads can also improve performance under blast loads as well as behavior with respect to progressive collapse.

Optimization under multiple hazards imposes multiple sets of constraints, all of which are applied simultaneously to the nonlinear programming problem (NLP) associated with the design. Among the applications of the optimization procedure is the reduction of the carbon footprint inherent in the energy embodied in structures via the minimization of an objective cost function that may include the cost of carbon emissions. A case study presented in [1] and [3] considers the

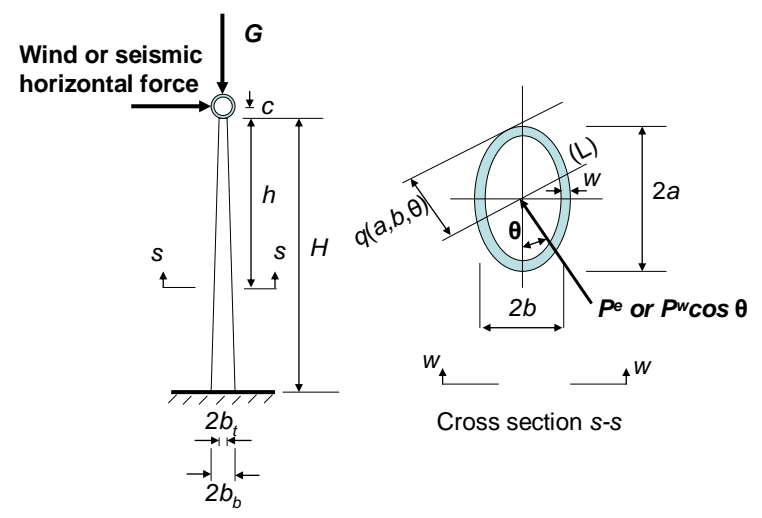

Figure 34. Vertical view and cross section of column. structure of Figure 34, representing one in a long row of columns free at the top and fixed at the base that support a pipe filled with water. The water is heated by ground-level, computer-controlled rotating mirrors that focus reflected solar rays onto the pipe. The resulting heat is used for power generation. The multi-hazard optimal design problem for this case study consists of determining the design variables in such way that the structure is guaranteed to sustain both wind and seismic loads and its cost is minimized. This problem was formulated as an NLP and solved by an interior point method.

[1] C. Crosti, D. Duthinh, J. Harris, L. T. Phan, F. A. Potra and E. Simiu, Wind engineering in a multi-hazard context: probabilistic, synergy, and optimization issues, in Proceedings of the 13th International Conference on Wind Engineering, IAWE, Amsterdam, The Netherlands, 2011 (8 pages).

[2] J. R. Hayes, S. C. Woodson, R. G. Pekelnicky, C. D. Poland, W. G. Corley and M. Sozen, Can Strengthening for Earthquake Improve Blast and Progressive Collapse Resistance?, Journal of Structural Engineering 131 (2005), 1157-1177.

[3] E. Simiu, F. A. Potra and D. Duthinh, Methodological and Probabilistic Issues in Multi-hazard Engineering Design, in Applications of Statistics and Probability in Civil Engineering (M. Faber, J. Koehler and K. Nishijima, eds.), CRC Press, Leiden, 2011, 836-839.

\section{Alternative Methods of Latent Fingerprint Enhancement and Metrics for Comparison}

\section{Alfred S. Carasso}

This work is a contribution to the FY 2012 research project Metrics for Manipulation and Enhancement of Forensic Images sponsored by the NIST Deputy Director for Laboratory Programs. That project is primarily focused on the processes used for digital image enhancement of poor quality latent fingerprints left unintentionally at a crime scene. To facilitate this study, law enforcement forensic experts provided NIST with a database of fingerprint image pairs, before and after enhancement. Such enhancements result exclusively from Adobe Photoshop image processing, which is the methodology preferred by law enforcement professionals.

In recent years, skepticism has occasionally been expressed about the reliability of such digital enhancements of latent fingerprints $[1,2]$. Of major concern is the possibility that the particular combination of Photoshop steps used in the enhancement process may inadvertently eliminate vital fine scale information, information that might exclude a suspect. Accordingly, 


\section{ALTERNATIVE ENHANCEMENTS OF LATENT PRINT (A)}

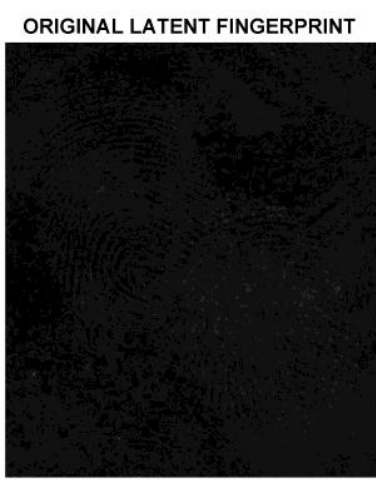

FORENSIC PHOTOSHOP PROCESS

MATLAB CONTRAST ADJUSTMENT
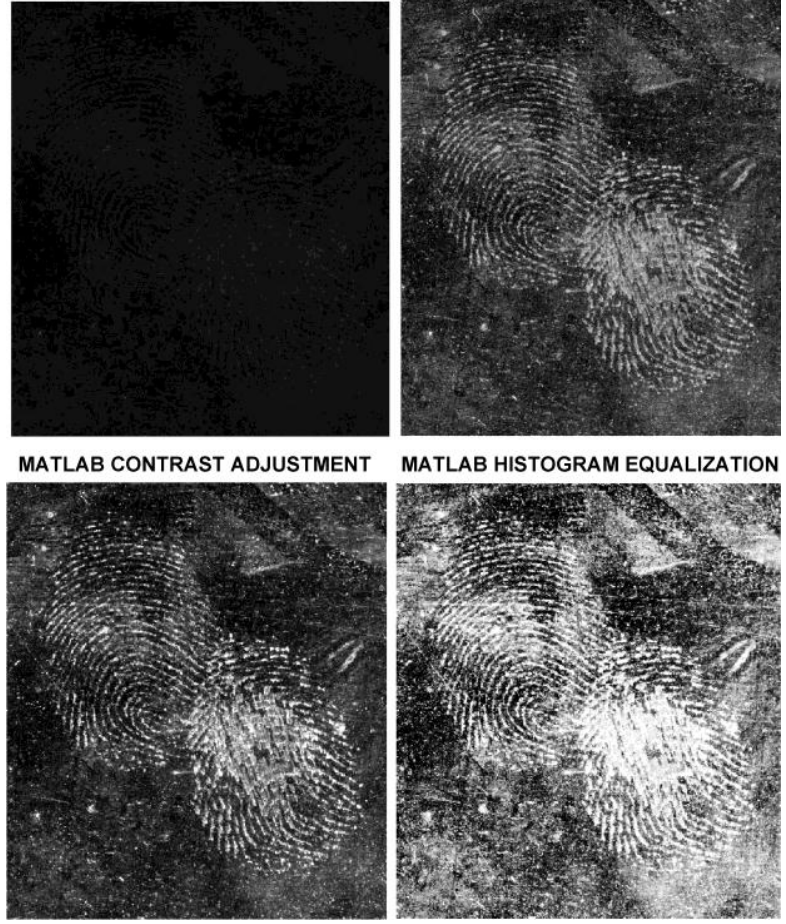

MATLAB HISTOGRAM EQUALIZATION

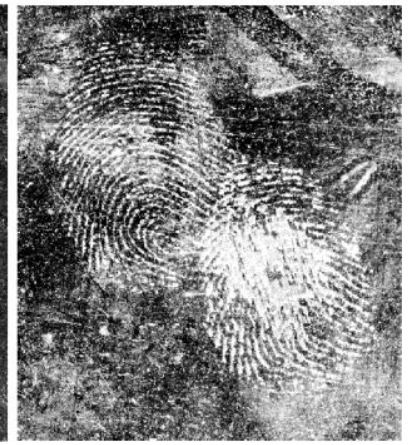

Figure 35. Enhancements of latent fingerprint. Original print, accompanying forensic Photoshop enhancement, and two alternative $M A T L A B$ enhancements. Significant enhancement of background features is apparent in histogram equalized image. This is reflected in the image gradient norms in Table 5.

a recently issued NIST Internal Report [7] explores alternative approaches to latent fingerprint enhancement that are independent of Photoshop. Consideration of such alternative enhancements, alongside the traditional Photoshop result, may better inform the subsequent, crucial, matching and identification procedure.

The proposed alternative methods yield reproducible results, and are based on the use of simple commands from such widely used scientific image analysis software packages as MATLAB [3], IDL [4], and PV-WAVE [5]. The uses and purposes of these commands are documented and illustrated in the accompanying user's manuals. In addition, [6] provides a thorough discussion of the theoretical ideas underlying the various enhancement techniques available in the above packages. Note that implementations vary among different packages, and the same enhancement technique can produce slightly different images in different packages. Such enhancement methods are designed to bring into better visual range potentially significant structures that are present in the original image, but not easily discernible.

Software routines that can be used with the above packages are included in [7]. These routines can perform contrast adjustment, Sobel filtering, unsharp

\section{REVERSE IMAGES IN ENHANCED LATENT PRINT (A)}

ORIGINAL LATENT FINGERPRINT
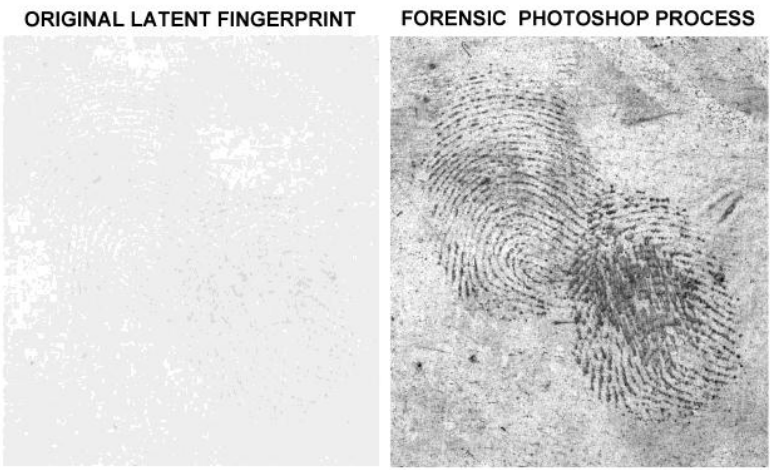

MATLAB CONTRAST ADJUSTMENT
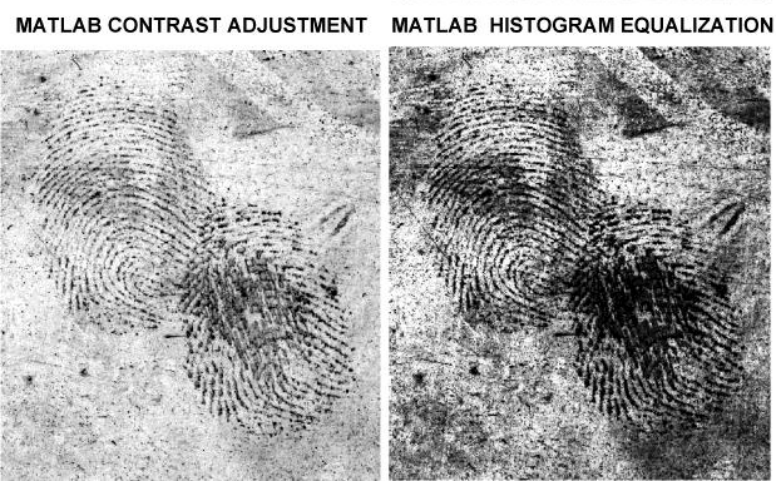

Figure 36. Reverse images in the latent print enhancements shown in Figure 35.

masking, and histogram equalization. The results of applying various these various enhancement methods to the same latent print, can be objectively compared using discrete $L^{1}$ and $L^{2}$ image norms, as well as $L^{1}$ and $L^{2}$ image gradient norms.

[1] F. McRoberts and S. Mills, Digitized Prints Can Point Finger at the Innocent, Chicago Tribune, January 3, 2005.

[2] F. McRoberts and S. Mills, US Seeks Review of Fingerprint Techniques, Chicago Tribune, February 212005.

[3] Image Processing Toolbox User's Guide, The MathWorks, Inc. Natick, MA 01760, 2012.

[4] ITTVIS Interactive Data Language (IDL), http://www.exelisvis.com/IDL.aspx.

[5] PV-Wave 7.5 Image Processing Toolkit User's Guide, Visual Numerics, Inc. Houston, TX 77042.

[6] W. K. Pratt, Digital Image Processing, Wiley-

Table 5. Image metrics in enhancements of latent print (A).

\begin{tabular}{|l|r|r|r|r|}
\hline Image & $\|f\|_{1}$ & $\|f\|_{2}$ & $\|\nabla f\|_{1}$ & $\|\nabla f\|_{2}$ \\
\hline Original Latent Print (A) & 16 & 18 & 2600 & 3900 \\
\hline Forensic Photoshop (A) & 61 & 75 & 25000 & 36000 \\
\hline Matlab Contrast Adj. (A) & 60 & 80 & 25000 & 40000 \\
\hline Matlab Histogram Eq. (A) & 112 & 138 & 42000 & 60000 \\
\hline
\end{tabular}


Interscience, New York, 2012.

[7] A. S. Carasso, Alternate Methods of Latent Fingerprint Enhancement and Metrics for Comparing Them, NISTIR 7910, January 2013.

\section{Shape Analysis}

\section{Javier Bernal \\ Charles Hagwood (ITL SED) \\ John Elliott (NIST MML) \\ Michael Halter (NIST MML)}

Many cell-based biomedical studies rely on the analysis of cell populations in the form of image data. Among the features that can be extracted from image data, cell shape is one that uniquely identifies a cell population. Accordingly, cell shape is an attribute on which statistical inference can be based regarding the effect of treating a disease in a particular way. The statistical tests used this way then provide the ability to identify homogeneous cell populations, and to differentiate between treated and untreated cells.

Shape governs a cell's motility, division, taxonomy, and oftentimes shape changes are a precursor to disease. Shape is generated by the outline of an object. Besides being required to be closed, smooth and simple, shapes are required to possess additional attributes such as scale, translational, rotational and reparametrization invariance. A theoretical foundation has been developed for shape space, in which the space is treated as a Riemannian manifold, so that calculus and other analysis can be performed. In particular the geodesic distance and path between elements of the space can be computed. This distance is used as a dissimilarity measure in the statistical tests (see Figure 37).

This year, we continued our work in cell biology, using mathematical and statistical techniques as mentioned above to characterize and analyze cell populations. What makes this work different from traditional applications in applied mathematics is that our data are not points in Euclidean space, but closed contours of cells treated as points in a space with a Riemannian structure, i.e., shape space. The data come embedded in fluorescent images. In previous work, we analyzed algorithms for segmenting cell images, from which the cell contours are obtained. We found the $k$ means algorithm with $(k=4)$, under a variety of imaging conditions, to be the best algorithm of four algorithms considered: $k$-means, Canny, Otsu thresholding and watershed. We used the cumulative distribution function of misclassification errors to compare algorithms, a new method we have introduced to the field. This work was published in 2012 in the IEEE Transactions on Medical Imaging [1]. Next, test procedures to compare cell populations based on cell shape were found. Shape provides a unique contour descriptor up to the invariants mentioned above. With the geodesic distance between shapes as a dissimilarity measure between cells, three nonparametric statistical tests for equality of populations were adapted to geodesic distance. This work was submitted to IEEE Transactions on Medical Imaging [2] and is under review.

[1] C. Hagwood, J. Bernal, M. Halter, J. Elliott, Evaluation of Segmentation Algorithms on Cell Populations using CDF Curves, IEEE Transactions on Medical Imaging 31 (2012), 380-390.

[2] C. Hagwood, J. Bernal, M. Halter, J. Elliott, T. Brennan, Testing Equality of Cell Populations based on Shape and Geodesic Distances, in review.

\section{A Standard Candle for Extragalactic Metrology}

\section{Bert W. Rust \\ Katherine M. Mullen (UCLA)}

To use Type Ia supernovae as standard candles in extragalactic metrology, we have developed a provisional calibration of the luminosities of six well-observed light curves in nearby galaxies whose distances are well known. Accurate metrology of the light curves was enabled by fitting them with our recently developed model,

$$
L(t)=C_{1} W(t)+C_{2}\left[k_{1} N_{1}(t)+2.146 k_{2} N_{2}(t)\right]
$$

where

$$
W(t) \equiv W\left(t ; \alpha_{1}, \alpha_{2}, \alpha_{3}\right)
$$

is a three parameter Weibull probability density function used to represent the pulse shape for the nuclear fusion reactions that deposit ${ }^{56} \mathrm{Ni}$ in the supernova, $N_{1}(\mathrm{t})$ and $N_{2}(\mathrm{t})$ are the subsequent pulses in the nuclear decay chain

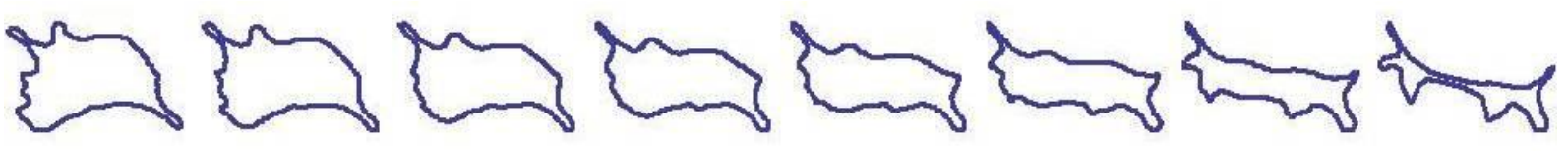

Figure 37. Geodesic path between two contours. 


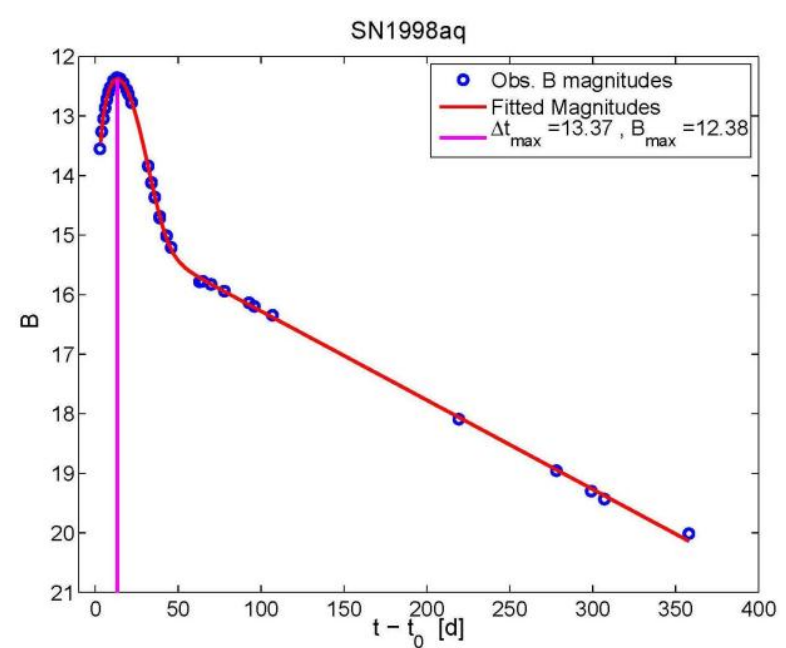

Figure 38. The light curve of SN1998aq. The time is measured in days since Ni deposition began. The discrete data points are observed apparent $B$ magnitudes and the smooth curve is the model fit. The vertical magenta line indicates the maximum brightness $B_{\max }$.

$$
{ }^{56} \mathrm{Ni} \stackrel{k_{1}}{\rightarrow}{ }^{56} \mathrm{Co} \stackrel{k_{2}}{\rightarrow}{ }^{56} \mathrm{Fe},
$$

and

$$
k_{1}=\frac{1}{8.764 \alpha_{4}}, \quad k_{2}=\frac{1}{111.42 \alpha_{4}} .
$$

The fitting parameters $C_{1}$ and $C_{2}$ are the constants which convert the relative abundances represented by the pulses into observed luminosities. The four nonlinear fitting parameters are: (1) $\alpha_{1}$, the time that ${ }^{56} \mathrm{Ni}$ deposition begins, (2) $\alpha_{2}$, a shape parameter, (3) $\alpha_{3}$, a scale parameter, and (4) $\alpha_{4}\left(0<\alpha_{4} \leq 1\right)$, an acceleration factor specifying the shortening of the half-lives of the two nuclear decays because of the extreme temperatures and densities inside the supernova. Strong evidence for such an acceleration effect was given many years ago by Rust, Leventhal and McCall [1], and recently confirmed by our work at NIST. The

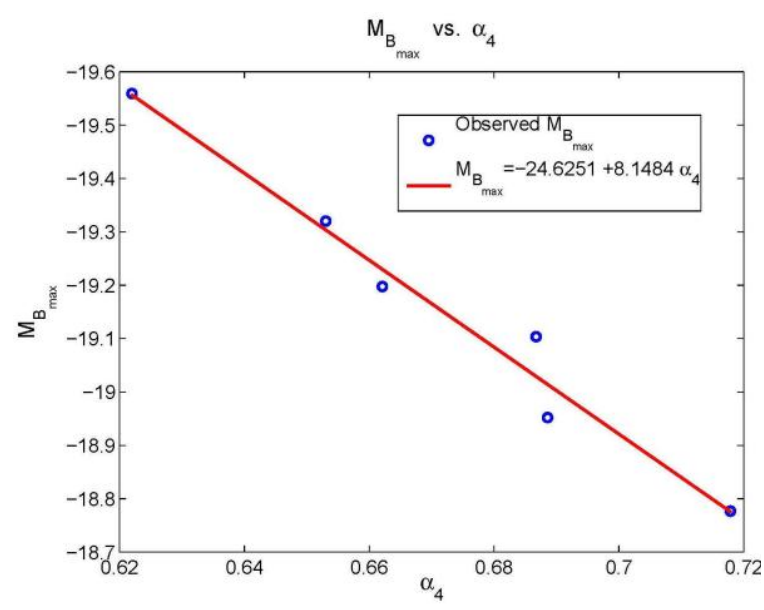

Figure 39. The correlation between $M_{B \max }$ and the decay acceleration parameter $\alpha_{4}$.
ODEs for the relative abundances can be written

$$
\begin{array}{lrl}
\frac{d N_{1}}{d t}=W(t)-k_{1} N_{1}, & N_{1}\left(\alpha_{1}\right)=0, \\
\frac{d N_{2}}{d t}= & k_{1} N_{1}-k_{2} N_{2}, & N_{2}\left(\alpha_{1}\right)=0, \\
\frac{d N_{3}}{d t}= & k_{2} N_{2}, & N_{3}\left(\alpha_{1}\right)=0,
\end{array}
$$

Using the nonlinear fitting program VARPRO ${ }^{14}$, we were able to get extremely good fits of the above model to the six light curves in the study. One of them is given in Figure 38. The other five fits were of similar quality, with all of them explaining more that $99 \%$ of the variance in the measured data $\left(R_{2}>0.99\right)$.

Since all six of the supernovae were in galaxies whose distances $D$ (in $\mathrm{Mpc}$ ), were already known, it was easy to convert the maximum apparent magnitudes $B_{\max }$ to maximum absolute magnitudes $M_{B \max }$ via the formula

$$
M_{B \max }=B_{\max }-5 \log _{10} D-25 .
$$

The luminosity calibration is possible because this measure of the absolute luminosity is very strongly correlated with the decay acceleration parameter $\alpha_{4}$. This correlation is illustrated in Figure 39. To estimate the distance to a faraway supernova: (1) measure its light curve, (2) fit the above model to estimate $\alpha_{4}$ and $B_{\max }$, (3) estimate $M_{B \max }$ from the correlation line in Figure 39, and (4) estimate $D$ from the preceding formula.

[1] B.W. Rust, M. Leventhal and S.L. McCall, Evidence for a Radioactive Decay Hypothesis for Supernova Luminosity, Nature 262 (1976) pp. 118-120.

\section{Unfolding Optical Spectrum Measurements}

\section{Bert W. Rust \\ Stephen W. Brown (NIST PML)}

The integral equations which must be inverted to correct optical spectrum measurements for scattered light in a certain spectrometer can be written

$$
\begin{array}{r}
y\left(\lambda_{i}\right)=\int_{220.0000}^{859.920} K\left(\lambda_{i}, \lambda_{L}\right) x^{*}\left(\lambda_{L}\right) d \lambda_{L}+\varepsilon\left(\lambda_{i}\right), \\
198.9388 \leq \lambda_{i} \leq 873.9835,
\end{array}
$$

where $y\left(\lambda_{\mathrm{i}}\right)$ the observed spectrum, $K\left(\lambda_{i}, \lambda_{L}\right)$ is the instrument response function, shown in Figure 40, $\varepsilon\left(\lambda_{i}\right)$ are random measurement errors, and $x^{*}\left(\lambda_{L}\right)$ is the unknown corrected spectrum. The black curve in Figure

${ }^{14}$ http://www.netlib.org/opt/varpro 


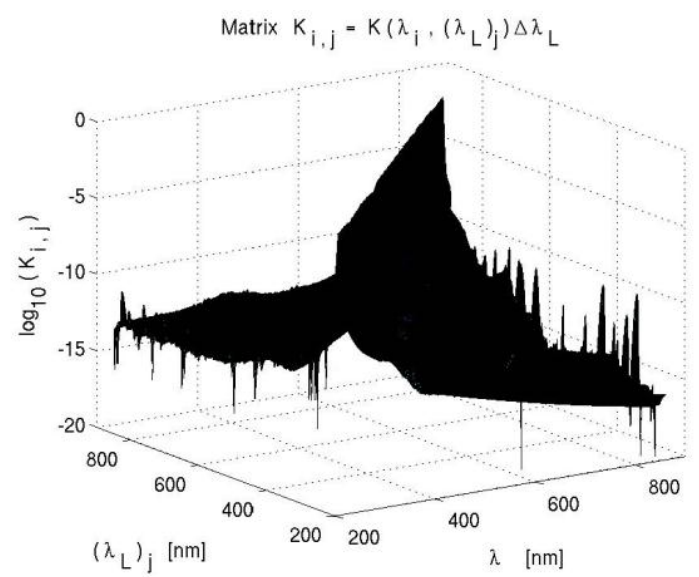

Figure 40. Instrument response matrix for the spectrometer, plotted on a log scale to show the small perturbations responsible for producing the scattered light.

41 is a mathematically computed test spectrum $x^{*}\left(\lambda_{L}\right)$ comprised by a linear combination of two Lorentzians, and the blue curve is the instrument's response $y^{*}(\lambda)$ that would be obtained if no random measurement errors were present. When the integral equations are discretized (with a $1024 \times 1024$ matrix $\boldsymbol{K}$ ), the result is a linear regression problem

$$
y=\boldsymbol{K} x^{*}+\varepsilon, \quad \varepsilon \sim N\left(0, \boldsymbol{S}^{2}\right),
$$

where $S^{2}$ is the positive definite covariance matrix for the measurement errors. If there were no measurement errors, it would be easy to compute the corrected spectrum by

$$
x^{*}=\boldsymbol{K}^{-1} y^{*} .
$$

The results of that calculation are shown in Figure 42.

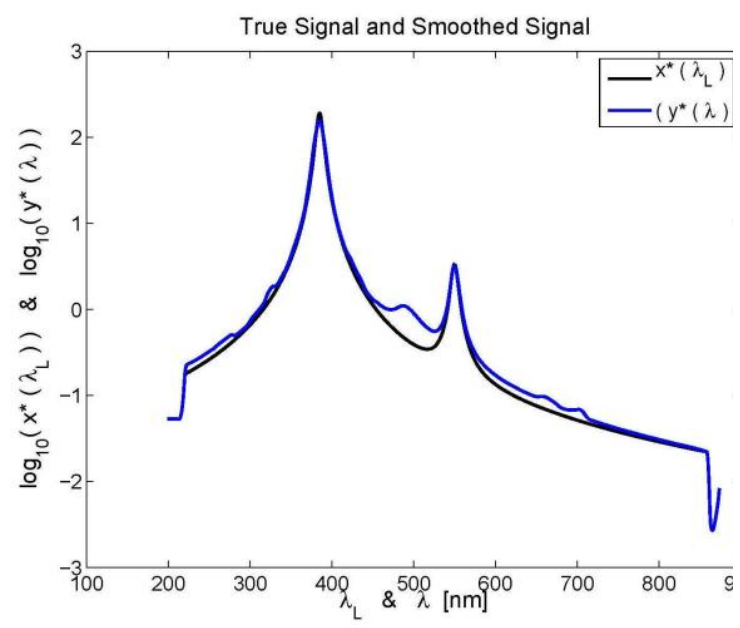

Figure 41. The instrument response $y^{*}$ to a mathematical test spectrum $x^{*}$ in the absence of measurement errors. The difference between the two curves is the scattered light produced by the spectrometer.
The outcome would have been much different if any random measuring errors had been added to $y^{*}$. Even with rounding errors so small that they changed $y^{*}$ only in the 5th or 6th digit, the resulting estimate of $x^{*}$ would have oscillated wildly between positive and negative values. One way often used to stabilize such problems is to impose a non-negativity constraint on them, i.e., to replace the least squares problem,

$$
S S R=\min _{x}\|y-\boldsymbol{K} x\|^{2},
$$

with

$$
S S R=\min _{x \geq 0}\|y-\boldsymbol{K} x\|^{2} .
$$

The Matlab Optimization Toolbox contains two commands, fmincon and lsqnonneg which purport to solve the latter problem, but as seen in Figure 43, our initial experiments show that they both fail when used to estimate $x^{*}$. Since these routines cannot provide good estimates when there are no measurement errors in the observed $y(\lambda)$, they should not be trusted in real problems when the observations do contain measurement errors.

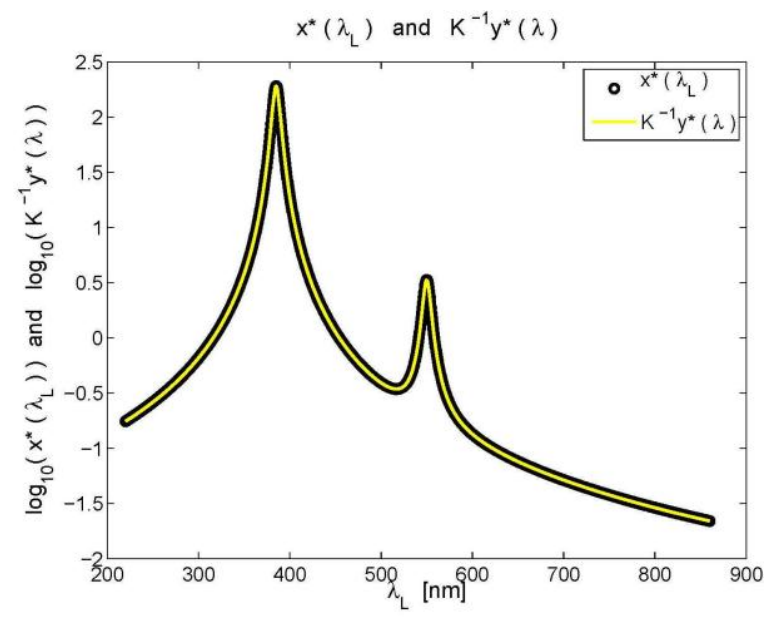

Figure 42. The true spectrum $x^{*}$ and the estimated spectrum $\boldsymbol{K}$ ${ }^{l} y^{*}$. The two vectors agree to around 5 significant digits, so they are graphically identical. 


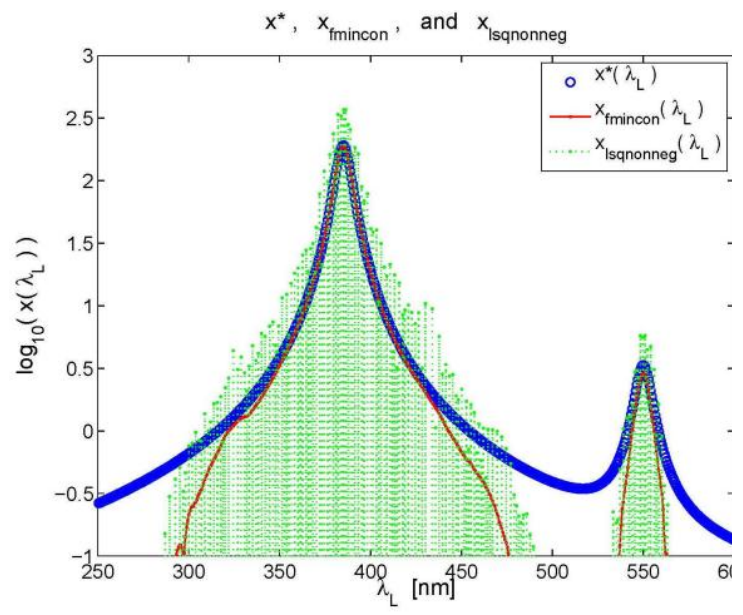

Figure 43. Two Matlab estimates of $x^{*}$. Most of the elements in the vector estimate $x_{\text {lsqnonneg }}$ are exact zeros, and most of them on the estimate $x_{\text {fmincon }}$ are numbers of order $10^{-16}$. 


\section{High Performance Computing and Visualization}

Computational capability is advancing rapidly. This means that modeling and simulation can be done with greatly increased fidelity (e.g. higher resolution, more complex physics). However, developing large-scale parallel applications remains highly challenging, requiring expertise that application scientists rarely have In addition, the hardware landscape is changing rapidly, and so new algorithmic techniques must constantly be developed. We are developing and applying facilities and expertise of this type for application to NIST problems. Large scale computations and laboratory experiments invariably produce large volumes of scientific data, which cannot be readily comprehended without some form of visual analysis. We are developing the infrastructure necessary for advanced visualization of scientific data, including the use of $3 D$ immersive environments and applying this to NIST problems. One of our goals is to develop the 3D immersive environment into a true interactive measurement laboratory.

\section{Rheology of Dense Suspensions}

\author{
William George \\ Steven Satterfield \\ Marc Olano \\ Judith Terrill \\ Nicos Martys (NIST EL) \\ Edward Garboczi (NIST EL) \\ Pascal Hebraud (CNRS/ESPCI, France)
}

See feature article, page 28.

\section{Visualization and Modeling of RF Propagation in Body Area Networks}

\author{
John Hagedorn \\ Kamran Sayrafian \\ Judith Terrill
}

See feature article, page 32 .

\section{Modeling and Visualization of Cement Paste Hydration and Microstructure Development}

\author{
William George \\ Steven Satterfield \\ Judith Terrill \\ Joel Muskin-Pierret \\ Jean-Loup Traore \\ Jeffrey Bullard (NIST EL) \\ Edward Garboczi (NIST EL) \\ Paul E. Stutzman (NIST EL)
}

http://www.nist.gov/itl/math/hpcvg/hydrationhpc.cfm http://www.nist.gov/itl/math/hpcvg/hydrationvis.cfm

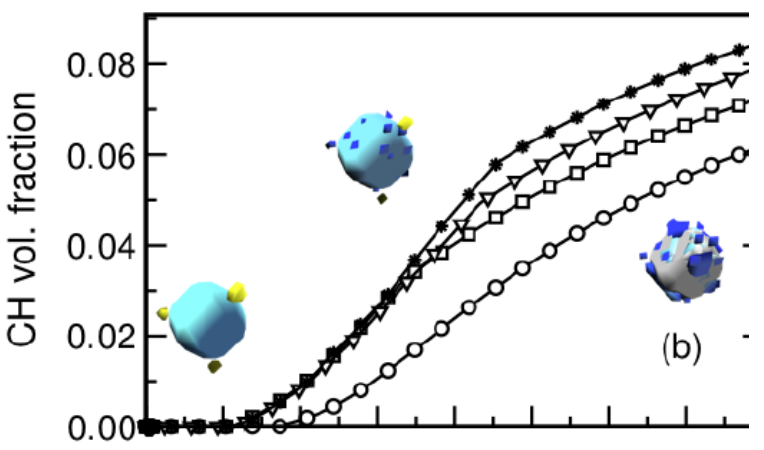

Figure 44. Plot of time variation of: $b$ ) portlandite for $C 3 S$ and C3S + gypsum systems. The vertical dotted lines represent the approximate beginning of acceleration for the systems with and without gypsum. Microstructure shown at $0 h, 3 h$, and $7 h$ for the $\mathrm{C} 3 \mathrm{~S}+2.5 \%$ gypsum system, with light blue $=\mathrm{C} 3 \mathrm{~S}$, yellow $=$ gypsum, dark blue $=$ portlandite, and gray $=\mathrm{C}-\mathrm{S}-\mathrm{H}$.

When cement powder is mixed with water, the hydration process that transforms the paste from a fluid suspension into a hardened solid involves complex chemical and microstructural changes. Understanding and predicting the rates of these changes is a longstanding goal. Computational modeling of the hydration of cement is challenging because it involves a large number of coupled nonlinear rate equations that must be solved in a highly irregular three-dimensional spatial domain. To address these challenges we are applying a new computational model called HydratiCA, which has several advantages. HydratiCA uses stochastic cellular automata algorithms to simultaneously model reaction and transport phenomena in 3D. This allows us to track the detailed kinetics and equilibria that occur in a diverse range of cementious systems.

Parallelization of the model is important so that we can simulate systems that are large enough to be realistic, avoiding finite size effects, and still be able to complete the simulations in a reasonable amount of time. Over the course of the simulation time, a series of data volumes is produced at the time values of interest. Visualization of the output is important both for 
validation and to understand the results.

This year we extended the computational model and ran a number of validation studies, including the creation and coalescence of void space in the simulation. We also analyzed the Message Passing Interface (MPI) calls in HydratiCA to aid in removing inefficiencies. Finally, we successfully implemented and tested checkpointing.

We have used HydatiCA to investigate the kinetic effect of sulfate incorporation and the possible implications for the determination of the "optimum sulfate." We ran computer simulations for two kinds of systems: $\mathrm{C}-\mathrm{S}-\mathrm{H}+$ gypsum + portlandite, and $\mathrm{C} 3 \mathrm{~S}$ with gypsum varying between $0 \%$ and $7.5 \%$ by mass of solids. The simulations indicated that, for the mechanisms that were assumed, sulfate ions should cause a shortening of the initial period of slow reaction as well as an overall increase in the hydration rate when C-S-H is able to incorporate sulfate ions. Some published experimental data indicate a lengthening of the slow reaction period when sulfates are present, so these assumed mechanisms may need to be re-evaluated.

In the coming year we will study the rate controlling mechanism for early age cement hydration. The results of these simulations will provide new insights into hydration mechanisms, will help resolve some long-standing controversies in the literature, and will pave the way toward better measurement science that will lead to improved standard test methods for early age concrete properties, as called for in a recent roadmap for hydration kinetics research published jointly by NIST and the Federal Highway Administration.

[1] L. Valentini, J. W. Bullard, G. Artioli, J.-L. Traore, S. G. Satterfield, J. E. Terrill, and M. Magistri, Role of Sulfate Incorporation into $\mathrm{C}-\mathrm{S}-\mathrm{H}$ during Cement $\mathrm{Hy}-$ dration: Results from Numerical Modeling of the System C3S + Gypsum, in preparation.

[2] J. W. Bullard, D. P. Bentz, E. J. Garboczi, S .G. Satterfield, P. E. Stutzman, L. Valenti, and G. Artioli, "Simulating Solution Chemistry and Phase Evolution in Early-age Cement Pastes", invited presentation at the ACI Spring 2012 Convention, Dallas, TX, March 18-22, 2012.

[3] L. Valentini, G. Artioli, J. W. Bullard, and S. G. Satterfield, "Modeling the Effect of Calcium Sulfate on C3S Hydration: Implications for the Origin of the Optimum Sulfate", Advances in Cement-Based Materials: Characterization, Processing, Modeling and Sensing, Austin, Texas, June 10-12, 2012.

[4] J. J. Biernacki, J. W. Bullard, D. Constantiner, M. Juenger, J. H. Cheung, W. Hansen, R. D. Hooton, A. Luttge, and J. Thomas, Paving the Way for a More Sustainable Concrete Infrastructure: A Vision for Developing a Comprehensive Description of Cement Hydration Kinetics, NIST SP 1138, November 2012.

\section{Nano-structures, Nano-optics, and How to Control Exciton Fine Structure with Electric Fields}

\author{
James S. Sims \\ John Kelso \\ Garnett W. Bryant (NIST PML) \\ http://www.nist.gov/itl/math/hpcvg/nanohpc.cfm \\ http://www.nist.gov/itl/math/hpcvg/nanovis.cfm
}

Research and development of nanotechnology, with applications ranging from smart materials to quantum computation to biolabs on a chip, has the highest national priority. Semiconductor nanoparticles, also known as nanocrystals and quantum dots, are one of the most intensely studied nanotechnology paradigms. Nanoparticles are typically $1 \mathrm{~nm}$ to $10 \mathrm{~nm}$ in size, with a thousand to a million atoms. Precise control of particle size, shape and composition allows one to tailor charge distributions and control quantum effects to engineer properties completely different from the bulk and from small clusters. As a result of enhanced quantum confinement effects, nanoparticles act as artificial, man-made atoms with discrete electronic spectra that can be exploited as light sources for novel enhanced lasers, discrete components in nanoelectronics, qubits for quantum information processing, and enhanced ultrastable fluorescent labels for biosensors to detect, for example, cancers, malaria or other pathogens, and to do cell biology.

We are working with the NIST Physical Measurement Laboratory to develop computationally efficient large scale simulations of such nanostructures, and also to develop immersive visualization techniques and tools to enable analysis of highly complex computational results of this type. The electrical and optical properties of semiconductor nanocrystals and quantum dots are studied. In the most complex cases this entails modeling structures with on the order of a million atoms. Highly parallel computational and visualization platforms are critical for obtaining the computational speeds and analysis tools necessary for a systematic, comprehensive study.

This year the work was focused on modulating and controlling the optical properties of self-assembled quantum dots using electric fields, culminating in a paper submitted to the Physical Review Letters [1]. Calculations were carried out on NIST's 4,404processor Linux cluster.

In addition to the electric field work, we have also added a parameter to the code which handles the imaginary part of the magnetic field parameters (electric field parameters are real) and there are now data input files for strain, electric fields, and magnetic fields. We are now doing new runs (and new research) incorporat- 
ing the effect of magnetic fields to what has already been investigated without including the effect of magnetic fields. This year we have completed testing the changes to the nanotech code for these imaginary parameters, and it is giving reasonable looking results. We have also redone some results from last year on bigger dots than we have been able to do before, and we are getting much better looking results than before. As the computational model has been extended to handle more complex and larger systems by including not only the nanostructures but also the substrate and environment around them, parallel processing has become even more of a necessity. Both shared memory OpenMP parallelization and distributed memory MPI are employed.

In the coming year the work will be focused on modulating and controlling the optical properties of self-assembled quantum dots via external magnetic fields using this new feature of our nanostructure codes.

[1] G. W. Bryant, N. Malkova, and J. Sims, Electric-field Control of Exciton Fine Structure: Atomic Scale Manipulation of Exchange Splitting, in review.

\section{High Precision Calculations of Fundamental Properties of Few- Electron Atomic Systems}

\author{
James Sims \\ Stanley Hagstrom (Indiana University) \\ M. B. Ruiz (University of Erlangen)
}

NIST has long been involved in supplying critically evaluated data on atomic and molecular properties such as the atomic properties of the elements contained in the Periodic Table and the vibrational and electronic energy level data for neutral and ionic molecules contained in the NIST Chemistry WebBook. Fundamental to this endeavor is the ability to predict, theoretically, a property more accurately than even the most accurate experiments. It is our goal to be able to accomplish this for few-electron atomic systems.

The mathematical intractability of the Schrödinger equation has prevented the computation of accurate numerical solutions for atomic and molecular systems until recently. In the past two decades, there have been breathtaking improvements in computer hardware and innovations in mathematical formulations and algorithms, leading to "virtual experiments" becoming a more and more cost-effective and reliable way to investigate chemical and physical phenomena.

The essential flaw in theoretical methods is their inability to correctly describe the mathematical cusp behavior of many-body wave functions in regions of close electron proximity, the so-called electron cusp region of the exact electronic wave function [1-3]. This flaw leads to incomplete accounting of instantaneous, short-range correlation among electrons. We are undertaking the theoretical development of our hybrid Hylleraas-CI wave function method to solve the electron cusp problem and bring sub-chemical accuracy to atomic systems with more than two electrons. Our Hy$\mathrm{CI}$ method has been employed over the past two years to explore its utility for both three electron lithium systems and the four electron beryllium atom. In the case of lithium, we have computed four excited states of the lithium atom to two orders of magnitude greater than has been done before [4].

At the four electron level, in calculations published in the Physical Review A: Atomic, Molecular, and Optical Physics [5], we have demonstrated the ability of Hy-CI calculations to achieve $0.1 \mu \mathrm{h}$ accuracy for beryllium. This represents one of the highest levels of accuracy ever achieved in quantum computations with more than three electrons. Familiar chemical electron affinities and ionization energies involve these nonrelativistic energies. To get truly accurate chemical properties like these it is important to go beyond the 0.1 microh accuracy to somewhere close to the nanohartre level we were able to achieve for lithium. To enable Hy-CI calculations to do this, we have been investigating more flexible atomic orbital basis sets, better configuration state function filtering techniques to control expansion lengths, and improved four election integral techniques. We will be employing the results of these improvements to our methodology in a much bigger beryllium calculation which will determine whether really accurate calculations on atoms with more than four electrons are feasible.

[1] T. Kato, On the Eigenfunctions of Many-particle Systems in Quantum Mechanics, Communications in Pure and Applied Mathematics 10 (1957), 151-177.

[2] R. T. Pack and W. B. Brown, Cusp Conditions for Molecular Wavefunctions, Journal of Chemical Physics $\mathbf{4 5}$ (1966), 556-559.

[3] W. Kutzelnigg and J. D. Morgan III, Rates of Convergence of the Partial-wave Expansions of Atomic Correlation Energies, Journal of Chemical Physics 96 (1992), 4484-4508.

[4] J. S. Sims and S. A. Hagstrom, Hylleraas-configurationinteraction study of the $2^{2} S$ ground state of neutral lithium and the first five excited ${ }^{2} S$ states, Physical Review A 80 (2009), 052507.

[5] J. S. Sims and S. A. Hagstrom, Hylleraas Configuration Interaction Study of the Singlet ${ }^{1} \mathrm{~S}$ Ground State of Beryllium, Physical Review A $\mathbf{8 3}$ (2011), 032518.

[Contributes to Virtual Measurements crosscut.] 

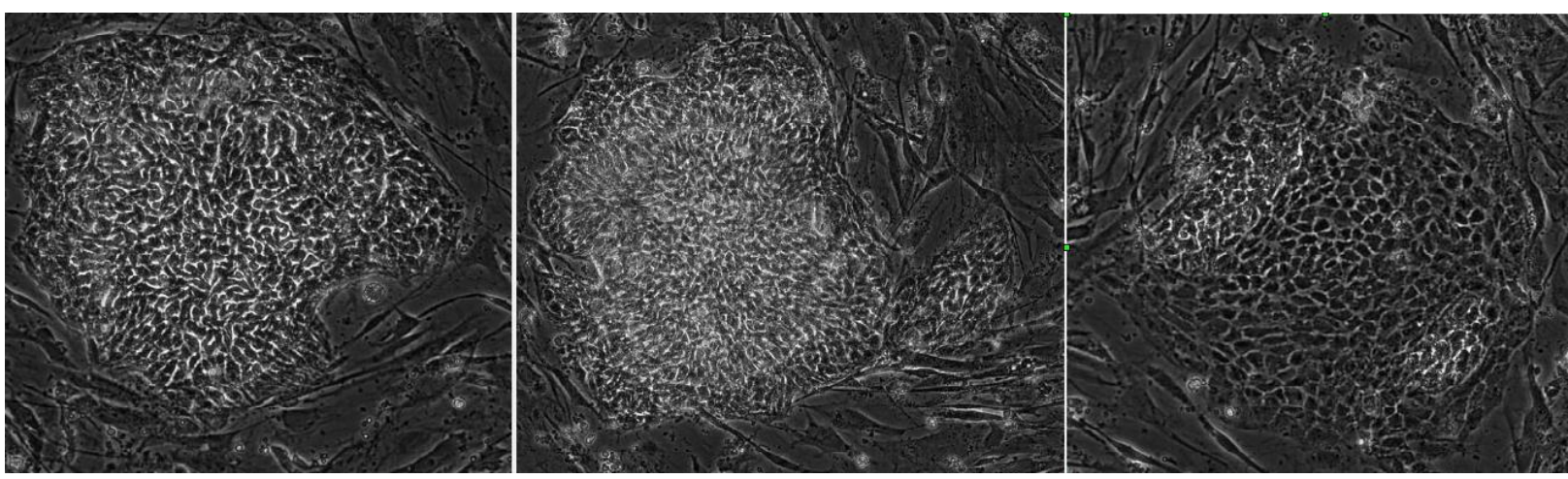

Figure 45. Examples of three types of stem cell colony images: healthy and undifferentiated cells, less healthy cells, and differentiated cells.

\section{Determination of Computational Features to Quantitatively Characterize Stem Cell Colony Quality}

\author{
Adele Peskin \\ Ya-Shian Li-Baboud (ITL SSD) \\ Peter Bajcsy (ITL SSD) \\ Michael Halter (NIST MML) \\ Anne Plant (NIST MML)
}

Working with biologists in MML and at the Lieber Institute, we have identified qualitative attributes of images of stem cell colonies that can help to automate the process of identifying colonies of healthy, undifferentiated cells. Our current reference data includes 182 image colonies that have been manually ranked on a scale of 1-5, with 1 representing colonies of differentiated cells, and 5 representing colonies of healthy undifferentiated cells. However, the rankings are subjective and often inconsistent from one person's assessment to another. So we are faced with two simultaneous questions: how to quantify image features in order to classify the colonies, and how to establish these classifications given that our reference data is sometimes inconsistent. Figure 45 shows a range of colonies from undifferentiated to differentiated.

We are working with a variety of image features, several of which are constructed specifically for this investigation, including a measure of contrast within a colony, which biologically represents how well individual cell edges are defined within the colony, and a measure of homogeneity, which represents the fraction of cells within the colony that are in the same biological state. Both of these measures are calculated from local standard deviations of pixel intensity, within small neighborhoods of each pixel, the size of which are representative of the size of individual cells within the colony. Other features include circularity of the colony, which indicates consistent growth within the colony, size of the colony, and smoothness of colony edges. A new method for colony segmentation was constructed, which was challenging due to the ambiguous edges of the colonies, in part due to another strain of cells that are grown along with the stem cells. Different approaches are currently being taken to classify the colonies given quantitative colony features, including decision trees based on sets of observer data where there is agreement [1].

[1] H., Witten, E. Frank, and M. A. Hall, Data Mining, Third Edition, Elsevier, Amsterdam, 2011.

\section{An Automated Method for Locating Phantom Nodules in Phantom CT Studies}

\author{
Adele Peskin \\ Alden Dima (ITL SSD) \\ Ganesh Saiprasad (ITL SSD)
}

http://www.nist.gov/itl/math/hpcvg/medimgvis.cfm

The Cancer Imaging Archive (TCIA) has a publicly available FDA database consisting of just over thousand CT scans intended to facilitate the assessment of lung nodule size estimation methodologies, the development of image analysis software, as well as a wide range of different analyses. The use of these scans would be greatly enhanced by the availability of phantom nodule location data that could be input into methods that require them. We have developed a new image processing method to locate the phantom nodules in these CT scans in order to avoid the need for laborious manual location prior to their use. We present a method for extracting the phantom nodules from the CT data, which involves phantom lung wall removal and separation of the phantom nodules from surrounding phantom blood vessels.

Nodules in the lung are classified according to their measured growth in CT scans taken over a period of time. Many prognostic techniques for cancerous 

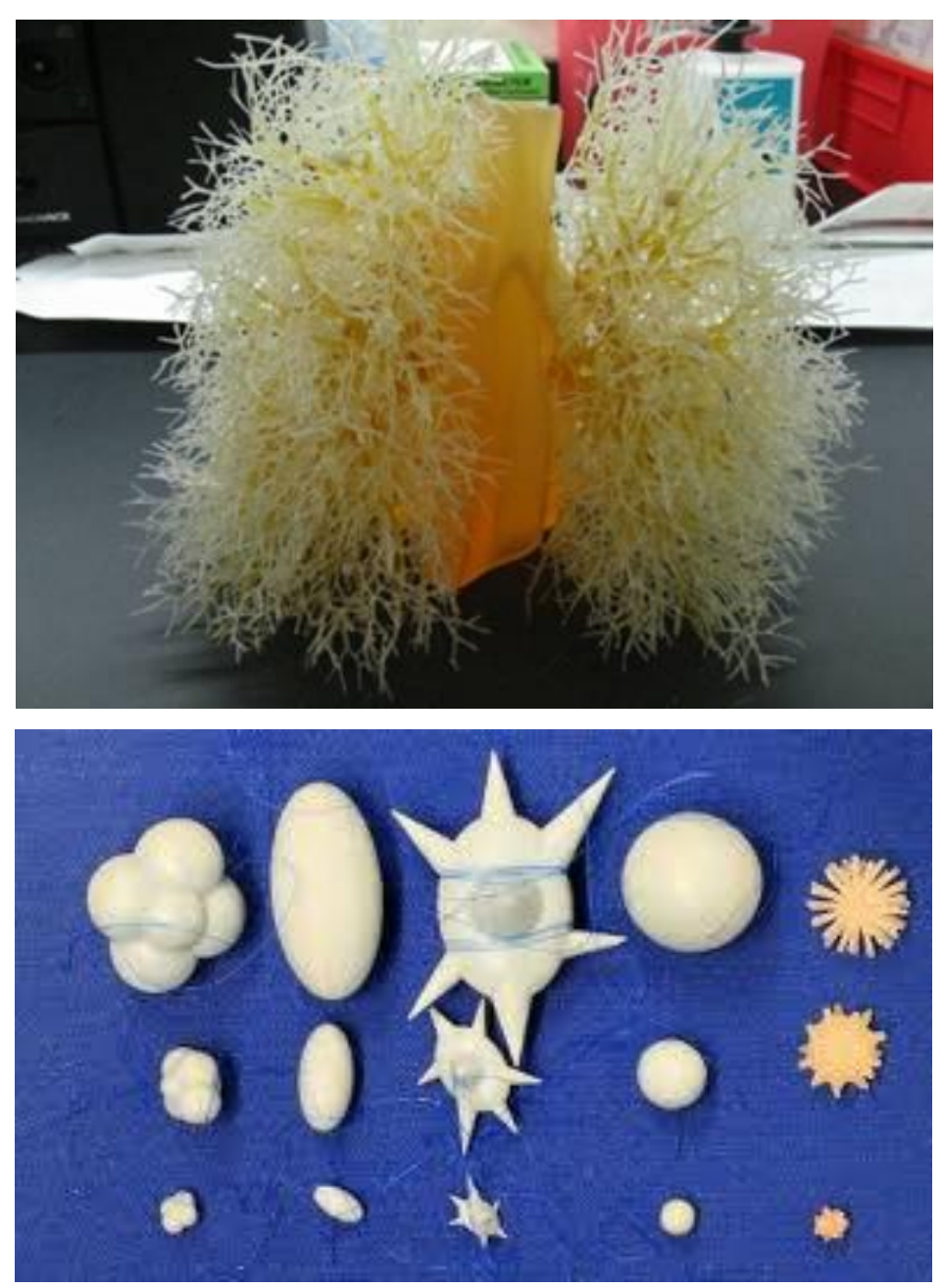

Figure 46. FDA lung phantom and nodules.

nodules depend on accurate volumetric measurement techniques. Fully automated volumetric techniques must be able to handle the complex task of separating nodules from the blood vessels and structures around them, and often attached to them. Many different techniques have been developed, both fully and semiautomated, and comparative studies have been done and are still underway [1,2]. The Radiological Society of North America's QIBA (Quantitative Imaging Biomarkers Alliance) Volumetric CT Study 3A is in the process of testing the variation and performance of algorithms for computing nodule volumes in CT lung nodule data sets ${ }^{15}$. The study is being currently being performed on phantom lung nodule data. The phantom data, generated at the FDA, is available via the TCIA for lung nodule size estimation assessment and image analysis software development ${ }^{16}$. However, the locations of the phantom nodules in these sets are not

\footnotetext{
15 http://qibawiki.rsna.org/index.php?title=Main_Page

16 https://wiki.cancerimagingarchive.net/display/Public/Phantom $\underline{+\mathrm{FDA}}$
}

known well enough to be used as inputs to methods which require them. Figure 46 shows a picture of the FDA lung phantom used to create the CT lung nodule data and the assortment of nodules.

The extraction of nodule locations in the lung data takes place in a series of three main steps: definition of the lung wall in the CT data, removal of the lung wall, and finally separation of the nodules from surrounding blood vessels. Locating the lung wall in the CT data takes advantage of the large intensity difference between the lung wall and the air inside the lungs. By locating sections of the higher intensity pixels that span the height of the lung, we extract sections of lung wall and locate nodules that are attached to the lung wall, which are then removed. Figure 47 shows an iso-surface of an entire CT lung data set at an iso-value of -100 Hounsfield units (HU), a small section of an iso-surface at $-100 \mathrm{HU}$ containing a nodule attached to the lung wall, and a sample of a lung wall extraction. After lung wall removal, higher intensity pixels are clustered, and nodules are separated from blood vessels according to the sizes and geometries of remaining pixel clusters.

Some results are shown in Figure 48, for a data set containing nodules with two different densities, in the $0 \mathrm{HU}$ and -600 $\mathrm{HU}$ ranges. We tested our nodule extraction methods against 40 sets of data for which manually selected reference data was available. For the thin-sliced data sets, where the slice thickness is approximately equal to the pixel-pixel distance within a slice, our algorithm correctly located $96.4 \%$ of the nodules in all of the sets, with a false positive selection in only $2.55 \%$ of the data. We are currently processing 1200 sets of FDA CT lung data for a variety of data mining projects.

[1] A. C. Jirapatnakul, A. P. Reeves, T. V. Apanasovich, A. M. Biancadi, D. F. Yankelevitz, and C. I. Henschke, Pulmonary Nodule Classification: Size Distribution Issues, in Proceedings of the International Symposium on Biomedical Imaging, 2007, 1248-1251.

[2] Kostis, W.J., Reeves, A.P., Yankelevitz, D.F., Henschke, C.I. Three-Dimensional Segmentation and GrowthRate Estimation of Small Pulmonary Nodules in Helical CT Images, IEEE Transactions on Medical Imaging 22:10 (2003).

[3] A. P. Peskin, A. Dima, and G. Saiprasad, Anthropomorphic Thoracic Phantom CT Studies, in Proceedings of the International Conference on Image Processing, Computer Vision, and Pattern Recognition, Las Vegas, July 16-19, 2012. 

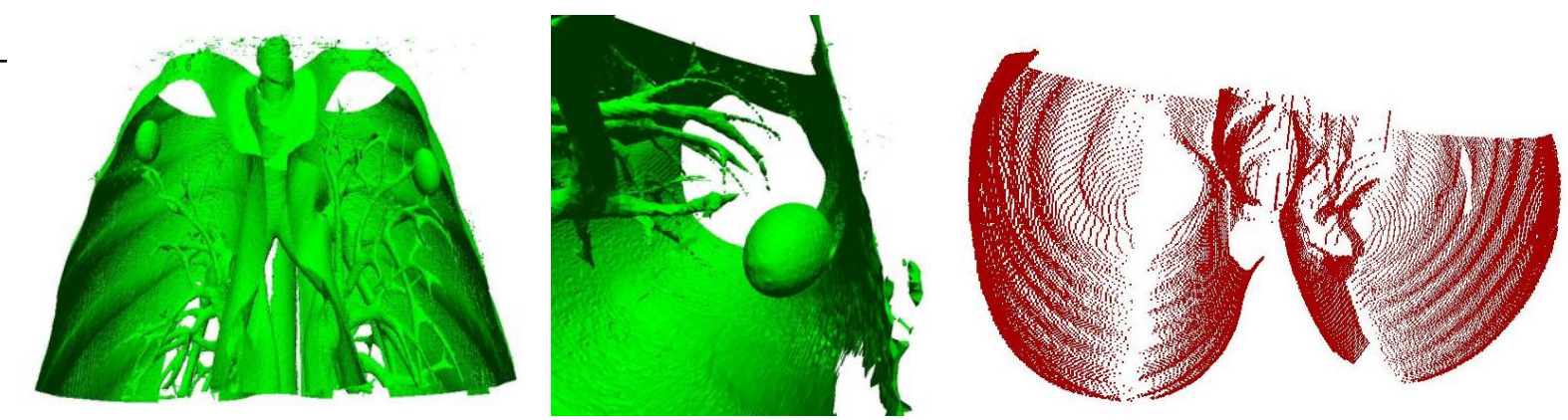

Figure 47. Iso-surface at -100 HU of an entire data set; close-up of nodule attached to wall; lung wall from a CT data set consisting of 119 slices, each $2.5 \mathrm{~mm}$.
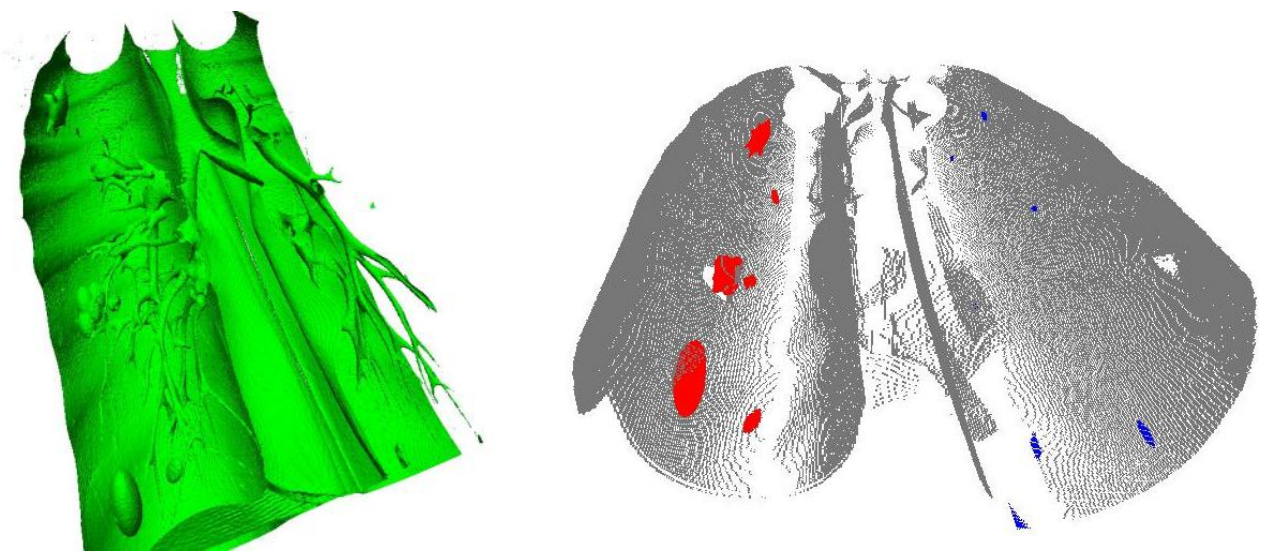

Figure 48. Isosurface at -100 Hounsfield units of a CT data set and the corresponding lung wall in grey and nodules in red (O HU nodules) and blue (-600 HU nodules).

\section{Declarative 3D Graphics}

\section{Sandy Ressler}

Manipulating and presenting 3D computer graphics as native elements of web pages is a once elusive goal that is now becoming a reality. Working with the Web3D Consortium and the Declarative 3D for Web Architecture Community Group of the World Wide Web Consortium (W3C), we have produced a number of demonstrations and an extensive tutorial illustrating the power and practicality of declarative markup for 3D.

Declarative, as opposed to imperative, markup describes the geometric objects of interest which are then interpreted by the web browser and rendered to the screen. Graphical objects such as "spheres", "cones", and so on, are treated in exactly the same way that "paragraphs", "headings" and other page elements are treated. These graphical objects appear in the web page's Document Object Model (DOM) just like regular page elements. This allows 3D application developers to use the rich infrastructure of web applications, including many widely used JavaScript frameworks, directly for their applications. The current implementation of declarative $3 \mathrm{D}$ that we use is called
X3DOM, developed primarily by Franhoffer and the Web3D Consortium.

We have produced a collection of demonstrations that embed declarative 3D graphics into HTML-based slide shows. These demonstrations are available at a NIST website ${ }^{17}$ and were demonstrated at the W3C

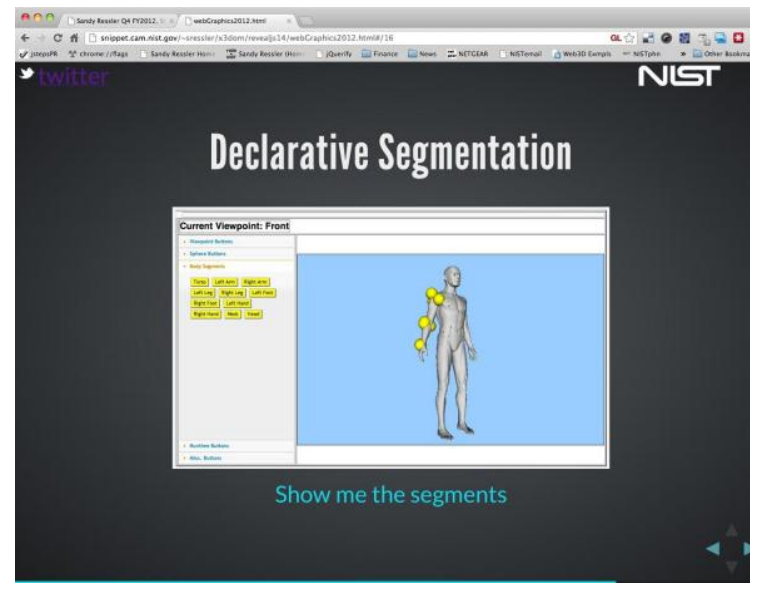

Figure 49. Declarative $3 D$ Graphics embedded in an HTML slide show.

17 http://math.nist.gov/ SRessler/dec3Dslides.html 
Technical Plenary Advisory Committee (TPAC) 2012 in Lyon France [1]. We also produced an extensive tutorial presented at the Web3D Conference in August of 2012 [3]. The tutorial ties together the use of X3DOM with jQuery and HTML5, and demonstrates many of the technical possibilities of declarative markup. These include the ability to dynamically generate user interface components that smoothly integrate with 3D graphics; the ability to associate semantics with graphical elements; and the ability to associate web based events with graphical models.

Finally we used the application of human anthropometry as a test-bed to demonstrate the use of declarative markup for a specific application [2].

[1] S. Ressler, Slides, Filters and Mobile Oh My! (Demonstrations of HTML and Declarative 3D Integration), 1st International Workshop on Declarative 3D for the Web Architecture, April 17, 2012, Lyon, France.

[2] S. Ressler, Web-based Declarative 3D Graphics for Anthropometry Visualization and Education, The Graphical Web 2012, September 13, 2012, Zurich, Switzerland.

[3] S. Ressler, Not Your Mother's Web3D: Integrating X3DOM, jQuery and HTML5, Web3D 2012, August 4, 2012, Los Angeles, CA.

\section{Information Visualization for Cloud Computing}

\section{Sandy Ressler \\ Kevin Mills (ITL ANTD)}

As part of the Measurement Science for Complex Information Systems project we produced a series of visualizations with the explicit goal of producing results quickly so as to be meaningful to the researchers during their period of inquiry and to influence the direction of the research. The visualization approach was to take the data from the researchers (K. Mills) and process it via a set of $\mathrm{R}$ scripts which generated the individual frames of the animation. Initially a set of 2D animations was produced for each of the 12 experiments. In addition a set of 3D animations, also using R, was produced.

An interactive VRML-based visualization was produced, with which the user can interact in real time (for individual time frames); see Figure 50. The user can spin the data object in real time, and more informatively, when the cursor is placed over the sphere, the fitness value appears as a popup. The color also corresponds to fitness.

Finally, an interactive web site using a new information visualization library called $\mathrm{d} 3$.js was used to implement a set of parallel coordinates to visualize the quantities of all the variables; see Figure 51. As you

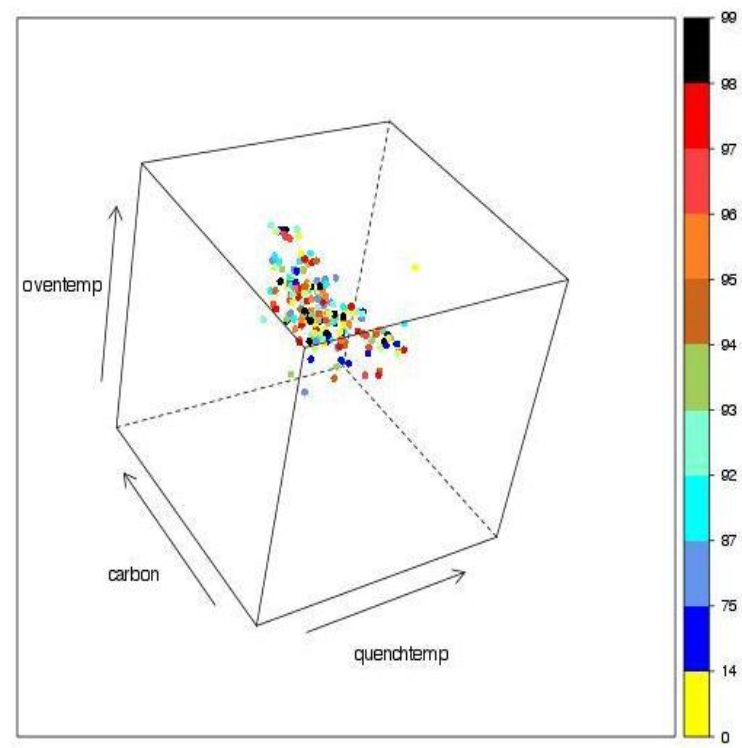

Figure 50. Genetic Algorithm visualization results illustrating convergence to high fitnesss.

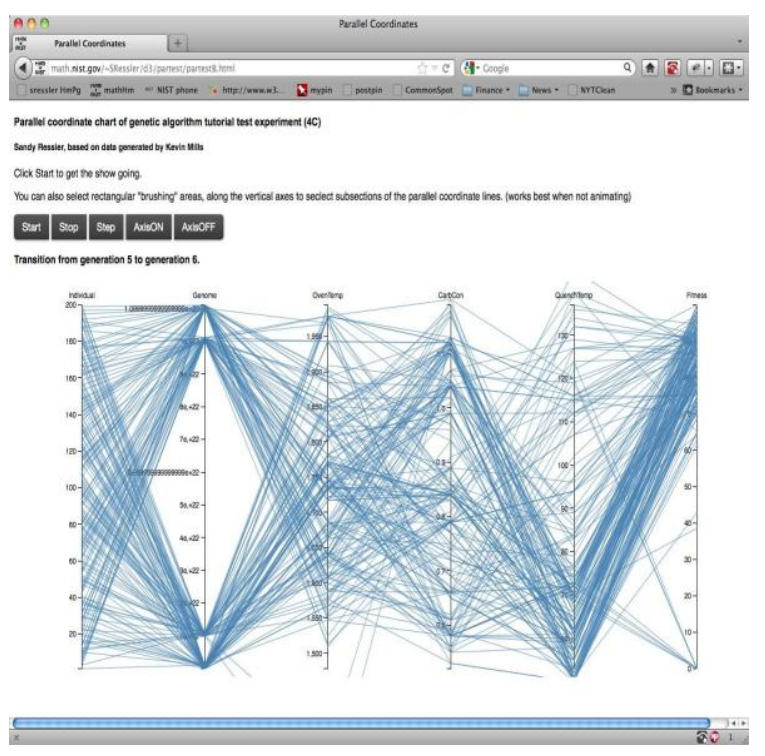

Figure 51. Parallel coordinate display of genetic algorithm variables.

watch the animation ${ }^{18}$ (taking place in real-time in the web browser) move through successive generations, you can observe the increase in fitness.

We plan on continuing to work with the research team of the Measurement Science project ${ }^{19}$, which is now focused on characterizing resource utilization for cloud computing.

\footnotetext{
18 http://math.nist.gov/ SRessler/d3/partest/partestB.html

19 http://www.nist.gov/itl/antd/emergent behavior.cfm
} 


\section{Interactive Visualization and Analysis Tools for the National Vulnerability Database}

\author{
Judith Terrill \\ Styvens Belloge \\ Sandy Ressler \\ Michael Ekstrom \\ Johathan Ibers \\ Harold Booth (ITL CSD)
}

The National Vulnerability Database (NVD) is the U.S. government repository of standards based vulnerability management data. It includes security checklists, security related software flaws, misconfigurations, product names, and impact metrics. This data enables automation of vulnerability management, security measurement, and compliance. Updated daily, it is available on the web as XML data. We are currently developing visualization and analysis tools to facilitate use of and insight into the NVD.

This year we created a schema for the XML data and created a tool to import the data into an SQLite database. The database is updated nightly after the new vulnerabilities are added to the website. We created a front end visualization and analysis tool, NVDsql, to facilitate study of the database. We developed a series of interactive web based visualizations using the Data Driven Documents (D3) tool. We gave a variety of D3 demos at the NIST booth at the 8th Annual IT Security Automation Conference, October 3-5, 2012, Baltimore MD. See Figure 52 for an example. In the coming year, we will be expanding our D3 tool set with the goal of incorporating these into the NVD web site when it undergoes its planned upgrade.

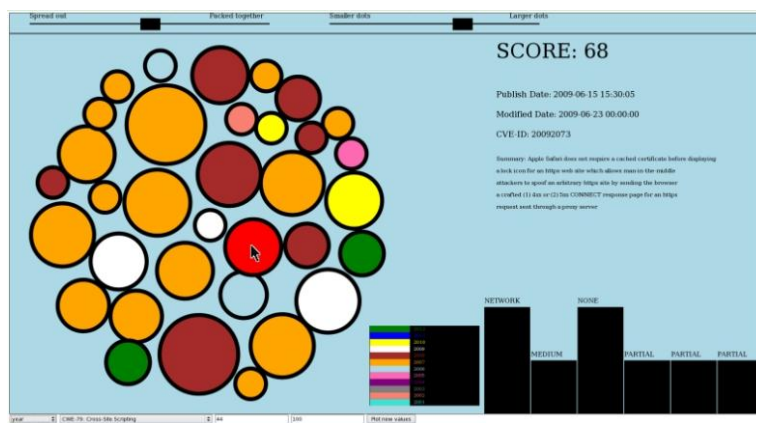

Figure 52. A dynamic visualization of the cases of cross site scripting with score above 43. The circle radii are based on the score of each case, with the smallest being 50 and the largest being 93. There is additional color coding for the year (user selected attribute). A user can select an individual case and see details on it. The red circle is currently selected and the information on this case is shown on the right.

\section{WebGL Enhancements for DLMF Visualization}

\author{
Sandy Ressler \\ Brian Clanton (University of Rochester) \\ Marc Olano
}

WebGL is a relatively new technology that enables web browsers to display OpenGL type 3D graphics natively on web pages. This allows users to create sophisticated graphics that take advantage of hardware GPU chips to produce high performance graphics displayed right in the web page. Using the new HTML5 "canvas" element one can place these graphics in the page, and most importantly, these graphics are portable and interoperable on most computing and web browser platforms. The NIST Digital Library of Mathematical Functions (DLMF) currently uses older visualization technology to display graphics. Interoperability is problematic, and maintenance is highly labor intensive. In addition to interoperability issues, we wanted to demonstrate more advanced capabilities and some of the visual potential of applying WebGL to the future visualization needs of the DLMF.

We have created a DLMF Model Viewer ${ }^{20}$ that displays the surfaces in a variety of different shadings; see Figure 53. The viewer has the ability to place a clipping plane at an arbitrary angle, an ability that had never been explored in the DLMF project.

With the successful conclusion of this demonstration, the next steps are to determine if this capability can be added to the production version of the DLMF. In addition, we anticipate improving the usability and visualizations of the model viewer as precursors to the production DLMF web site.

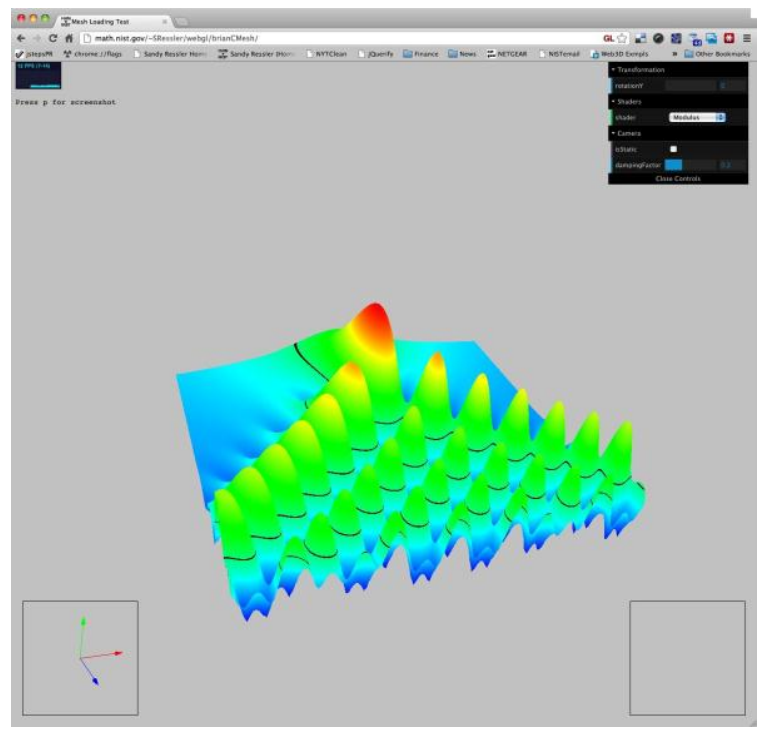

Figure 53. The DLMF Model Viewer.

${ }^{20}$ http://math.nist.gov/ SRessler/webgl/brianCMesh/index.html 


\section{Scientific Software}

Modern science and engineering in general, and modern measurement science in particular, require a wide variety of software tools for scientific discovery, exploration, and analysis. As scientific inquiry becomes deeper and more specialized, so must the supporting software tools. The goal of this work is to develop critical software tools that support measurement science at NIST, as well as computational science and engineering at large.

\section{OOF: Finite Element Analysis of Material Microstructures}

\author{
Stephen A. Langer \\ Andrew C.E. Reid (NIST MML) \\ Günay Doğan (Theiss Research)
}

http://www.ctcms.nist.gov/oof/

The OOF Project, a collaboration between ACMD and MML, is developing software tools for analyzing real material microstructure. The microstructure of a material is the (usually) complex ensemble of polycrystalline grains, second phases, cracks, pores, and other features occurring on length scales large compared to atomic sizes. The goal of OOF is to use data from a micrograph of a real or simulated material to compute the macroscopic behavior of the material via finite element analysis. OOF is intended to be a general tool, applicable to a wide variety of microstructures in a wide variety of physical situations.

The currently available version of OOF is OOF2, which solves two dimensional time-dependent problems. Two versions were released this year, 2.1.6 and 2.1.7. These versions fixed bugs and improved OOF2's handling of electron backscatter diffraction data, which allows users to load experimentally measured crystalline orientation data directly into the program. OOF2 can now also generate pole figures, which are a way of viewing the orientation data, allowing users to check that the data was read correctly. (A number of parameters affect interpretation of the data, and OOF2's assumptions may not always match the assumptions of the software that produced the data.)

One major area of development this year was OOF3D, which solves three dimensional problems starting from three dimensional micrographs. A large fraction of the work on OOF3D involved writing and rewriting its graphics code. OOF3D's graphics uses the vtk library ${ }^{21}$, which is very powerful and often maddeningly difficult to use. Shortcomings in the graphics interface were brought to light by development of the test suite, and new visualization methods were required to determine if mesh modification routines were operating correctly.

\footnotetext{
21 http://www.vtk.org/
}

A second area of development this year has been image segmentation, although this work has not yet been integrated into a released version of OOF2. Image segmentation is the task of identifying distinct regions and structures in given images. It is critical to the analysis of material micrographs. The OOF approach to image segmentation is energy minimization, trying to match curves to region boundaries in images using special segmentation energies. In the last fiscal year, several computational procedures were developed and implemented, enabling the efficient and robust deployment of two very effective segmentation energies, the piecewise-constant Mumford-Shah model and the Geodesic Active Contour (GAC) model. The two models are useful for segmenting images with flat regions and images with distinct boundaries, respectively. The implementation of the GAC model contains procedures to compute high-quality edge indicator functions, reliable image derivatives and interpolation, and uses special components for the corresponding Newton's method for faster computation. The piecewise-constant model employs sophisticated meshing and integration routines that quickly compute statistics of arbitrary image regions. Key ingredients to this model are adaptive mesh refinement and statistical error estimators used to determine the right amount of work to obtain the result, taking into account the inherent limitations on the available information due to finite image resolution. Significant effort was invested into improving the numerical infrastructure as well. Extensive tests and profiling led to many improved components and achieved significant speed-ups, ranging between $3 \mathrm{X}-100 \mathrm{X}$.

The OOF team also worked on adding new features to OOF2. They completed the infrastructure for surface properties, and implemented a demonstration surface property with a prescribed flux on one side of the surface. This likely completes the prerequisites for OOF to support discontinuous surface effects such as those present in electrochemical problems. Also during this year, they made substantial progress on understanding the fundamentals of crystal plasticity, having worked through some simple example problems in other codes. These results form the beginnings of a validation suite, to ensure that the OOF implementation of crystal plasticity is correct. 

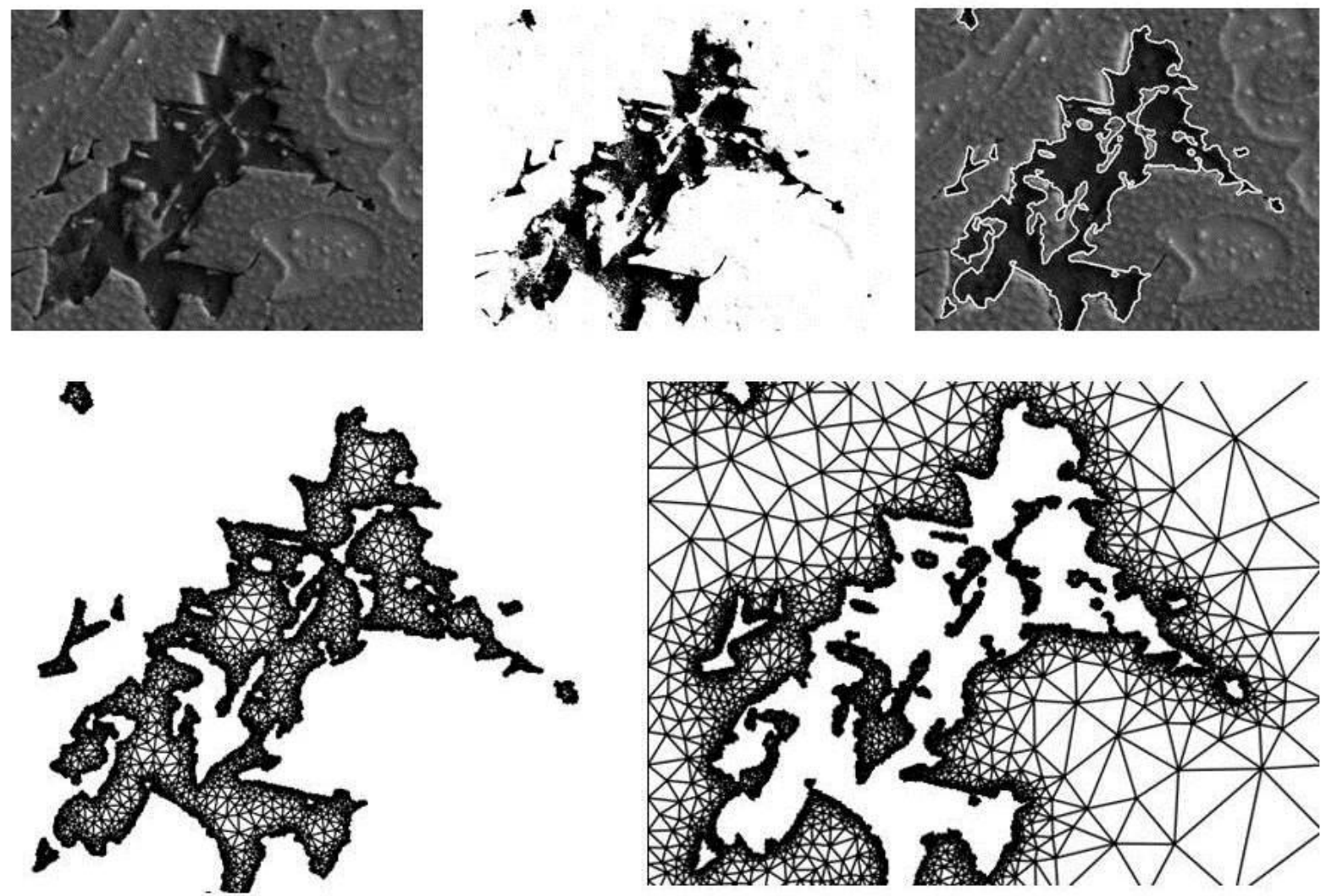

Figure 54. Segmentation and meshing of a micrograph: The top row shows the original input micrograph (left, courtesy of Dale Newbury, $M M L)$, its segmentation by thresholding (middle), a widely used method for image segmentation, and its segmentation with our implementation of the Chan-Vese model (right), which produces coherent regions unlike thresholding (which results in many unwanted speckles). The bottom row shows the meshes corresponding to the two phases of the micrograph. The meshes are of high quality and suitable for finite element simulations, and, they do not require any adjustment by the user. The meshes contain many fewer degrees of freedom compared to the original pixel grid, 10,131 and 11,006 elements respectively, whereas the input image has 819,200 pixels. Reduction of megapixel images to meshes of a few tens of thousands of high quality elements is typical in our experiments.

The OOF2 team has worked with developers on the nanoHUB site ${ }^{22}$ to update the version of OOF2 that is installed there. 244 people used OOF2 on nanoHUB this FY (October 2011 - September 2012).

OOF2 was downloaded from its home page 1,217 times between August 2011 and July 2012. 82,761 manual pages were read online during that period.

[1] Valerie R. Coffman, Andrew C. E. Reid, Stephen A. Langer, and Gunay Dogan, OOF3D: An Image-based Finite Element Solver for Materials Science, Mathematics and Computers in Simulation 82 (2012), 2951.

\section{Micromagnetic Modeling}

Michael Donahue

Donald Porter

Robert McMichael (NIST CNST)

June Lau (NIST MML)

http://math.nist.gov/oommf/

Advances in magnetic devices such as recording heads, field sensors, magnetic nonvolatile memory (MRAM), and magnetic logic devices are dependent on an understanding of magnetization processes in magnetic materials at the nanometer level. Micromagnetics, a mathematical model used to simulate magnetic behavior, is needed to interpret measurements at this scale. ACMD is working with industrial and academic partners, as well as with colleagues in the NIST CNST, MML, and PML, to improve the state-of-the-art in micromagnetic modeling.

Michael Donahue and Donald Porter in ACMD have developed a widely used public domain computer

22 http://www.nanohub.org/ 
code for doing computational micromagnetics, the Object-Oriented Micromagnetic Modeling Framework (OOMMF). OOMMF serves as an open, welldocumented environment in which algorithms can be evaluated on benchmark problems. OOMMF has a modular structure that allows independent developers to contribute extensions that add to the basic functionality of OOMMF. OOMMF also provides a fully functional micromagnetic modeling system, handling both two and three-dimensional problems, with sophisticated extensible input and output mechanisms. In fiscal year 2012 alone, the software was downloaded more than 5,000 times, and use of OOMMF was acknowledged in 186 peer-reviewed journal articles. OOMMF has become an invaluable tool in the magnetics research community.

Key new developments in OOMMF over the last year include:

1. Continued development on measurement, analysis, and improvement of the performance of multithreaded computations. Efforts included enabling more code modules to use the SSE instruction set where available, development of a multi-level thread launch and join control system, and techniques for striping data arrays across memory nodes of non-uniform memory access (NUMA) architectures.

2. Improvements to numeric accuracy when computing magnetostatic fields, particularly for computational cells with large aspect ratios, and when computing the angle between spins in adjacent cells, which is important in error and step-size control.

3. A uniaxial anisotropy energy that can vary with simulated time, or calculation stage.

4. Support for adding Zhang damping to the computed magneto dynamics.

5. A snapshot release delivering the latest developments to the micromagnetic community.

Ongoing work includes extension of periodic boundary condition support to two dimensions, and collaboration with the University of California, San Diego, on porting some OOMMF calculations to massively parallel graphical processing units (GPUs) with the support of the Materials Genome Initiative.

OOMMF is part of a larger activity, the Micromagnetic Modeling Activity Group (muMAG), formed to address fundamental issues in micromagnetic modeling through two activities: the development of public domain reference software, and the definition and dissemination of standard problems for testing modeling software. ACMD staff members are involved in development of the standard problem suite as well. The latest standard problem is an extension of a proposal [1] aimed at validation of simulations of spin momen- tum transfer in the "current parallel to plane" geometry. A solver that computes spin momentum transfer is based on one of two competing equations. The extensions to the proposed standard problem serve to illustrate where the two competing equations diverge in their predictions. Ongoing work includes development of OOMMF input files and post-processing scripts capable of computing the interpolated vortex position demanded by the terms of the problem.

In addition to the continuing development of OOMMF, the project also does collaborative research using OOMMF. The project played an instrumental role in the 2007 Innovation in Measurement Science project (with CNST, MML and PML), Magnetic Nanostructures for Post-CMOS Electronics. This work involved development of methods for modeling polycrystalline materials with magnetically thick grain boundaries. Recent work has used OOMMF to investigate the scaling of input fields of an s-shaped nanoscale magnetic logic device with device size [2]. In all, ACMD micromagnetic project produced two journal papers, three invited talks, and one conference presentation this past year.

[1] M. Najafi, et al., Proposal for a standard problem for micromagnetic simulations including spin-transfer torque, Journal of Applied Physics 105 (2009) 113914113921.

[2] G. S. Abo, Y.-K. Hong, B.-C. Choi, M. J. Donahue, S. Bae, J. Jalli, J. Park, J. Lee, M.-H. Park, and S.-H. Gee, Micromagnetic Computer Simulated Scaling Effect of S-shaped Permalloy Nano-element on Operating Fields for AND or OR Logic, IEEE Transactions on Magnetics 48 (2012), 1851-1855.

[3] M. J. Donahue, Micromagnetic Investigation of Periodic Cross-tie/Vortex Wall Geometry, Advances in Condensed Matter Physics (May 2012) 908692, 8 pages.

[Contributes to Virtual Measurements crosscut.]

\section{Parallel Adaptive Refinement and Multigrid Finite Element Methods}

\author{
William F. Mitchell \\ Marjorie A. McClain \\ Eite Tiesinga (NIST PML) \\ Paul Julienne (NIST PML) \\ Thomas Hanna (NIST PML) \\ John Villarrubia (NIST PML)
}

http://math.nist.gov/phaml

Finite element methods using adaptive refinement and multigrid techniques have been shown to be very efficient for solving partial differential equations. Adaptive refinement reduces the number of grid points by concentrating the grid in the areas where the action is, and 
multigrid methods solve the resulting linear systems in an optimal number of operations. Recent research has focused on $h p$-adaptive methods where adaptivity is in both the grid size and the polynomial order of approximation, resulting in exponential rates of convergence. $\mathrm{W}$. Mitchell has been developing a code, PHAML, to apply these methods on parallel computers. The expertise and software developed in this project are useful for many NIST laboratory programs, including material design, semiconductor device simulation, and the quantum physics of matter.

This year we completed a study of the performance of 13 proposed $h p$-adaptive strategies in terms of accuracy vs. degrees of freedom. For this experiment we gathered a collection of 12 test problems, most of which came from the numerical results section of papers on $h p$-adaptive refinement $[1,2,3,6,8]$. These problems contain a variety of difficulties including singularities, sharp peaks, wave fronts, oscillations and boundary layers. Most of them are parameterized to adjust the strength of the difficulty. We found that which strategy is most effective depends on the type of difficulty, and the accuracy requirements. The socalled reference solution methods performed well uniformly, but are very expensive computationally. Of the less expensive methods, the simple smooth prediction and type parameter strategies performed well most of the time. We have subsequently put effort into improving the efficiency of the code, and are now examining accuracy vs. computation time.

The development and application of PHAML continue to be of prime importance. As multicore computers begin to dominate the market, additional models of parallelism need to be implemented in scientific software. During the past year we continued the addition of OpenMP directives to PHAML to implement a shared memory model [7]. Not only is this appropriate for a multicore computer, but it can be combined with the previously implemented MPI message passing model to form a hybrid MPI/OpenMP model that uses shared memory parallelism on the multicore nodes of a cluster and message passing between the nodes.

PHAML was originally developed to solve elliptic partial differential equations in two spatial dimensions. We are now extending it to 3D, in support of collaborations with PML. This year we implemented the basic functionality, but still lack many features including high order elements and parallel execution.

There are two major collaborative efforts with PML that apply PHAML to their physical models.

- In collaboration with Eite Tiesinga and Tom Hanna, PHAML was used to calculate the bound states, scattering properties and dynamics of two dipolar molecules held in a cylindrically symmetric trap [4,5]. We have studied in detail the twobody energy spectrum, and shown for the first time that the trap may be used to actively control the strength of the interactions. In collaboration with Eite Tiesinga and Paul Julienne, we are currently extending these simulations to a 3D model.

- We began a new collaboration with John Villarrubia to apply PHAML to the modeling of scanning electron microscope images of samples that contain a mixture of conducting and insulating regions. John has a code that models electron scattering in materials, secondary electron production, and detection. We are coupling this code with PHAML, which will perform the finite element analysis to determine the electric fields that affect the image.

Future work will continue to enhance PHAML with additional capabilities, robustness and efficiency, implement and study some recently proposed $h p$-adaptive strategies, complete the extension of PHAML to 3D problems, and continue collaborations that use PHAML to solve NIST applications.

[1] W. F. Mitchell and M. A. McClain, A Survey of $h p$ Adaptive Strategies for Elliptic Partial Differential Equations, in Recent Advances in Computational and Applied Mathematics (T. E. Simos, ed.), Springer, 2011, 227-258.

[2] W. F. Mitchell and M. A. McClain, A Comparison of $h p$-Adaptive Strategies for Elliptic Partial Differential Equations, in review.

[3] W. F. Mitchell, A Collection of 2D Elliptic Problems for Testing Adaptive Algorithms, in review.

[4] T. M. Hanna, E. Tiesinga, W. F. Mitchell and P. S. Julienne, Resonant Control of Polar Molecules in an Optical Lattice, Physical Review A 85:2 (2012).

[5] T. M. Hanna, E. Tiesinga, W. F. Mitchell and P. S. Julienne, Bound states of interacting polar molecules in an optical lattice, 42nd Annual DAMOP Meeting, Atlanta, GA, June 2011.

[6] W. F. Mitchell and M. A. McClain, Comparison of $h p$ Adaptive Finite Element Strategies, Foundations of Computational Mathematics, Budapest, July 2011.

[7] W. F. Mitchell, Experience with MPI and OpenMP in an Adaptive FEM Code, SIAM Conference on Parallel Processing for Scientific Computing, Savannah, GA, February 2012.

[8] W. F. Mitchell, Comparison of $h p$-Adaptive Finite Element Strategies, George Washington University, Washington, DC, March 2012. 


\section{Digital Library of Mathematical Functions}

The special functions of applied mathematics are fundamental tools enabling modeling and analysis in all areas of science and engineering. To make effective use of such functions, practitioners must have ready access to a reliable source of information on their properties. The goal of this work is the development and dissemination of definitive reference data on the special functions of applied mathematics. The centerpiece of this effort is the DLMF, a freely available interactive and richly linked online resource.

\section{Digital Library of Mathematical Functions}

\author{
Daniel Lozier \\ Frank Olver \\ Ronald Boisvert \\ Marjorie McClain \\ Bruce Miller \\ Bonita Saunders \\ Abdou Youssef \\ Brian Antonishek (NIST ITL) \\ Charles Clark (NIST PL) \\ Adri Olde Daalhuis (University of Edinburgh)
}

http://dlmf.nist.gov/

Math and science go hand in hand. Purely mathematical developments find application in practical problemsolving in all fields of science as well as engineering, while cutting-edge science is a major driver of mathematical research. Often the mathematical objects at the intersection of math and science are mathematical functions. Effective use of these functions requires ready access to their many intricate properties. This need was capably satisfied for 50 years by the NBS Handbook of Mathematical Functions [1]. $21^{\text {st }}$ century needs, greatly enlarged since 1964, are being met by the NIST Digital Library of Mathematical Functions ${ }^{23}$ and its print companion, the NIST Handbook of Mathematical Functions [2]. The new handbook contains more than twice as many formulas as the old one, coverage of more functions, and an up-to-date list of references.

Collectively, the new handbook and website are called the DLMF. The website covers everything in the handbook and much more: additional formulas and graphics, interactive search, live zooming and rotation of 3D graphs, internal links to symbol definitions and cross-references, and external links to online references and sources of downloadable software.

The website is being updated regularly based on user feedback as well as continual review of the latest published research and tracking of emerging web technologies by the DLMF editors and staff. There have been five updates since public release in May 2010, two in calendar year 2012. The update on March 2 made several improvements affecting display of math and graphics, augmented the software index, and updated the help files. The October 1 update corrected 11 reported errors, four of which were math errors in formulas and the rest were relatively less important clarifications of math statements. It also included six new formulas, 36 new references to recently published literature, and a few textual clarifications and other minor improvements in the bibliography and index. This update is the first time formulas have been added to the original DLMF content. A complete record of changes since original release is available online ${ }^{24}$.

In August 2012 Dr. Adri Olde Daalhuis of the University of Edinburgh was added as a DLMF Mathematics Editor. Dr. Olde Daalhuis is (co-)author of three DLMF chapters. He has made substantial contributions to the five DLMF web updates, especially the fifth one, and he is actively engaging with the other four editors in planning for a second edition of the printed handbook, which will bring it up to date with respect to the website.

We are working with Cambridge University Press to maximize adoption of the DLMF as a replacement for the old Abramowitz and Stegun handbook. Sales to date of the Cambridge volume have exceeded 4,000 copies. Google Scholar currently lists about 400 citations. This number covers all academic fields, including physics, which in our experience is the source of many applications that use math functions. Finally, for calendar year 2012 there were more than 256,000 visits to the DLMF web site by some 140,000 unique visitors. Those visitors download more than 2 million individual DLMF web pages.

[1] M. Abramowitz and I. Stegun, eds., Handbook of Mathematical Functions with Formulas, Graphs and Mathematical Tables, Applied Mathematics Series 55, National Bureau of Standards, Washington, DC, 1964.

[2] F. Olver, D. Lozier, R. Boisvert, and C. Clark, eds., NIST Handbook of Mathematical Functions, Cambridge University Press, 2010.

${ }^{24}$ http://dlmf.nist.gov/errata 


\section{Mathematical Knowledge Management}

Bruce Miller

Joe Corneli (Open University, UK)

Deyan Ginev (Jacobs University, Germany)

Michael Kohlhase (Jacobs University, Germany)

Heinrich Stamerjohanns (Jacobs University, Germany)

Abdou Youssef (George Washington University)

Most of ACMD's current activity in Mathematical Knowledge Management (MKM) revolves around work-flows that use LaTeX markup as the document source, as was the case with the NIST Digital Library of Mathematical Functions (DLMF), for which we developed the LaTeXML [1] system. Newer applications continue to rely on the LaTeX markup familiar and convenient to authors in most heavily mathematical fields. The LaTeXML system converts that markup into XML and MathML for use on the web, with improved usability, reusability and accessibility.

We have reported previously on the arXMLiv project [2] based at Jacobs University. This project assembles a collection of workstations to convert, in parallel, the over half-million documents stored in Cornell's preprint server. Between the large volume of material and its sometimes haphazard markup quality, this corpus exercises the software to the utmost. The result however is not only a more convenient means of browsing the repository, but also an extremely useful data set for research in search, semantic analysis and other topics. Recent developments have improved the automation of RDFa (Resource Description Framework) metadata for the documents.

The development of a server-based Daemon [3] provided a web-service whereby other applications can convert LaTeX authored mathematics into usable forms. This opened up a number of interesting possibilities, including supporting mathematics in various Wikis. A particularly high profile Wiki containing mathematics is the Planet Math web site ${ }^{25}$. Planet Math is currently developing a beta version based on ideas from the Planetary project [4]. Here, the conversion to MathML should support the benefits of semantic representation of mathematics in terms of reuse, search and discovery of mathematical relationships.

Recent work has brought us close to realizing the conversion to SVG (Scalable Vector Graphics) of many forms of graphical markup used in LaTeX documents. While SVG may not provide the same level of accessibility to the truly blind as does MathML, it significantly improve the usability and scalability.

The real challenge, however, is ongoing work involving the generation of Content MathML, or semantic representation, of the mathematics. Always a

\footnotetext{
25 http://planetmath.org/
}

goal, and further prodded by the Planetary project, inferring the semantics of ambiguous markup is not necessarily always possible, short of full human intelligence (or AI?). Nevertheless, much can be inferred or clarified by augmenting the documents or, at least, recording and representing the ambiguities for later analysis. How such inferences are best done, on the one hand, and how to represent ambiguities, on the other, are continuing work [5]. Much infrastructural work within LaTeXML has been carried out to support such conversions and the interconnected parallel representations needed to make use of them.

All of these developments eventually lead back to DLMF. Truly reusable mathematics based on content MathML will allow importing to other software such as computer algebra or graphics systems, perhaps even automated validation. Capabilities such as the Planetary system could be used for mathematics-aware social media related to DLMF.

[1] B. Miller and A. Youssef, Technical Aspects of the Digital Library of Mathematical Functions, Annals of Mathematics and Artificial Intelligence 38 (2003), 121136. And see http://dlmf.nist.gov/.

[2] H. Stammerjohanns, M Kohlhase, D. Ginev, C. David, and B. Miller, Transforming Large Collections of Scientific Publications to XML, Mathematics in Computer Science 3:3 (2010), 299-307.

[3] D. Ginev, H. Stammerjohanns, B. Miller, and M. Kohlhase, The LaTeXML Daemon: Editable Math on the Collaborative Web, Lecture Notes in Computer Science 6824 (2011), 292-294.

[4] M. Kohlhase, et al., The planetary System: Web 3.0 \& active documents for STEM, Procedia Computer Science 4 (2011), Special issue: Proceedings of the International Conference on Computational Science (ICCS) (Mitsuhisa Sato, et al., eds.)

[5] D. Ginev, The Structure of Mathematical Expressions, Master's Thesis, Jacobs University, 2011.

\section{Visualization of Complex Function Data}

\author{
Bonita Saunders \\ Brian Antonishek (NIST ITL) \\ Qiming Wang \\ Bruce Miller \\ Sandy Ressler \\ Daniel Lozier \\ Frank Olver
}

Any visualization of complex function data must be both accurate and accessible if it is to be of use to a mathematical or physical scientist trying to understand the behavior of the function. The goal of our work is to create highly accurate visualizations that can be viewed 
by users on most widely used computer platforms. A properly constructed adaptive computational grid can enhance the fidelity and usability of a visualization by bringing out key features of the function such as zeros and poles while minimizing file size. We have had some success in developing such grids using a composite map consisting of tensor product B-splines together with variational techniques. Preliminary results were presented at the biannual meeting of the International Society for Grid Generation (ISGG 2012) [1].

We have attacked the accessibility problem in a variety of ways. We successfully created PDF files with embedded interactive 3D graphics from the DLMF. Qiming Wang and Brian Antonishek worked with Bruce Miller to integrate this work into the DLMF so that the visualizations are embedded automatically whenever the DLMF PDF is regenerated. The resulting file can be viewed on any platform using an Adobe Acrobat Reader.

Currently, DLMF website visualizations are rendered using two common technologies for 3D graphics on the web: Virtual Reality Modeling Language (VRML) and X3D. Although users may view the visualizations by downloading a free plugin, we have not found an acceptable viewer for the Linux operating system that easily handles all the custom features. Consequently, we have been looking at WebGL as an alternative. WebGL is a JavaScript API for rendering interactive 3D graphics inside an HTML5 web page without the use of a plugin. Primarily due to excellent work by Qiming Wang, we have made outstanding progress toward creating WebGL versions of the DLMF visualizations. Expanding on work originally done by Sandy Ressler, Qiming has successfully implemented in WebGL all the capabilities present in the DLMF visualizations, including alternative color maps, viewpoints, scaling and cutting plane animation. A sample rendering is shown in Figure 55.

WebGL visualizations are usable on Windows, Mac, and Linux platforms in most HTML5 compatible browsers with the exception of Internet Explorer (IE). Microsoft supports HTML5, but the company has indicated they have no intention of supporting WebGL because of security concerns. We are looking at a work-around using Chrome Frame or Flash which will allow a user to view a WebGL file even when attempting to access it through IE. Also, we are automating the transformation of the DLMF X3D files into the WebGL format and working with Bruce Miller to design the best way to integrate the files into the DLMF. For now, we expect WebGL to be another option in addition to the VRML and X3D formats, but in the future we expect to be able to drop the other formats when WebGL is more established.

[1] B. Saunders, Q. Wang, and B. Antonishek, Adaptive Composite B-Spline Grid Generation for Interactive 3D Visualizations, in Proceedings of MASCOT/ISGG 2012,

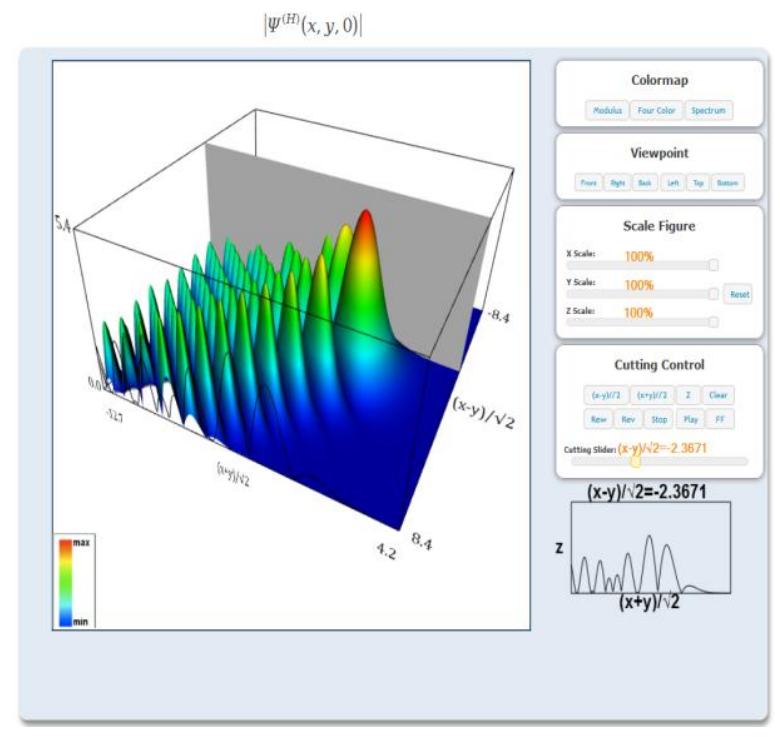

Figure 55. Modulus of hyperbolic umbilic canonical integral function $\left.\mid \Psi^{(\mathrm{H})} x, y, 0\right) \mid$.

Las Palmas de Gran Canaria, Spain, October 22-26, 2012.

[2] B. Saunders and Q. Wang, Tensor Product B-Spline Mesh Generation for Accurate Surface Visualizations in the NIST Digital Library of Mathematical Functions, Mathematical Methods for Curves and Surfaces, in $R e$ vised Selected Papers of the Seventh International Conference (MMCS 2008), Tonsberg, Norway, June 26July 1, 2008, Lecture Notes in Computer Science, Vol. 5862, Springer, 2010, 385-393.

[3] B. Saunders and Q. Wang, Tensor Product Grid Generation for Complex Surface Visualizations in a Digital Library of Mathematical Functions, Proceedings of the $11^{\text {th }}$ International Society for Grid Generation (ISGG) Conference, Montreal Canada, May 25-28, 2009, SV.3.

[4] Q. Wang, B. Saunders, and S. Ressler, Dissemination of 3D Visualizations of Complex Function Data for the NIST Digital Library of Mathematical Functions, in CODATA Data Science Journal 6 (2007) (Supplement on the Proceedings of the $20^{\text {th }}$ International CODATA Conference, Beijing, China, October 2006), S146- S154.

[5] B. Saunders, Q. Wang, From B-Spline Mesh Generation to Effective Visualizations for the NIST Digital Library of Mathematical Functions, Curve and Surface Design: Avignon 2006, Sixth International Conference on Curves and Surfaces, Avignon, France, Nashboro Press, 2007, 235-243. 


\section{DLMF Standard Reference Tables on Demand}

\author{
Bonita Saunders \\ Bruce Miller \\ Marjorie McClain \\ Daniel Lozier \\ Chris Schanzle \\ Annie Cuyt (University of Antwerp) \\ Stefan Becuwe (University of Antwerp) \\ Franky Backeljauw (University of Antwerp)
}

Researchers and mathematical software designers have long used tables of elementary and special function values for numerical exploration, software validation, algorithm development, or graphics production, but previously published tables often suffered from severe limitations: low precision, restriction to real arguments, limited number of arguments and parameters, and inability to accommodate alternate function definitions. The goal of our project is to provide a state of the art online system where these limitations become attributes which the user has some flexibility to specify. The system output will be function values with an accuracy certification.

The first phase of the project involves the integration of a front-end developed at NIST with high precision reference software developed by a research group at the University of Antwerp under the leadership of Dr. Annie Cuyt. Their software, which uses continued fractions as the main tool, establishes an error bound for the function computation. The group continues to add new functions to their package, but a substantial subset of the functions in the Digital Library of Mathematical Functions (DLMF) is currently covered.

On December 6-11, 2011 we hosted a workshop with Annie Cuyt's group to discuss the implementation of a prototype for the first phase. In less than two days Bruce Miller (ITL) and Stefan Becuwe (Antwerp) merged our frontend with the $\mathrm{C}++$ code of Cuyt's group to produce a working prototype with a small number of functions. During the two week meeting a selected group of testers at NIST and the University of Antwerp were given access to the system. We made some initial changes based on feedback from the testers. Additional changes were made over the following months after emails and numerous conference calls with Cuyt's group. New mathematical functions were added and the website interface improved. We added MathJax, a JavaScript display engine for mathematics, to make our web page math content accessible to users with browsers that don't support MathML. We implemented help features, including simple function descriptions and links to the appropriate pages of the DLMF. We are currently discussing the possibility of providing links to extensive documentation and refer-

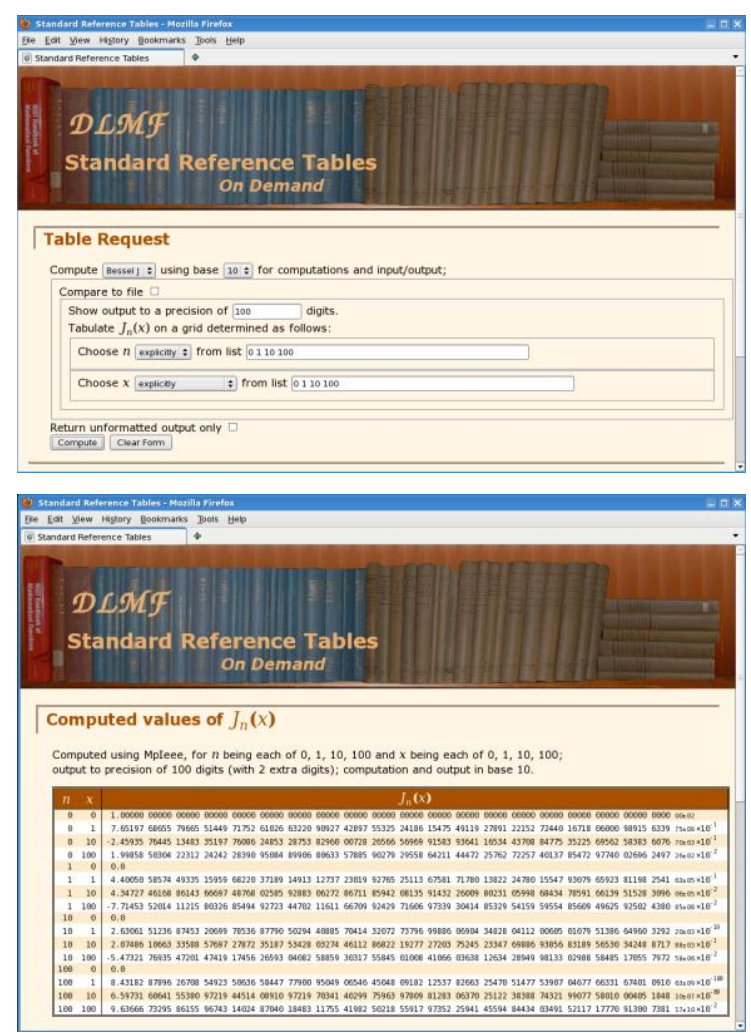

Figure 56. User interface to the Standard Reference Tables.

ences for users interested in a more in depth understanding of the function validation techniques being used.

Daniel Lozier and Annie Cuyt each presented invited talks on aspects of the project at the Workshop on Numerical Software: Design, Analysis, and Verification, Santander, Spain, July 4-6, 2012 [1] held in association with the yearly meeting of IFIP's Working Group 2.5 on Numerical Software. Over the next few months additional modifications and testing will be conducted before the public release of the first phase.

[1] F. Backeljauw, S. Becuwe, A. Cuyt, J. Van Deun, and D. Lozier, Validated Evaluation of Special Mathematical Functions, in Proceedings of Workshop on Numerical Software: Design, Analysis, and Verification, Santander, Spain, July 4-6, 2012, Science of Computer Programming, in review.

[Contributes to Virtual Measurements crosscut.] 


\section{Special Function Expansions and Definite Integrals Associated with Fundamental Solutions of Linear Partial Differential Equations}

\author{
Howard S. Cohl \\ Hans Volkmer (University of Wisconsin Milwaukee) \\ Ernie G. Kalnins (University of Waikato) \\ Gestur Ólafsson (Louisiana State University)
}

The concept of a function expresses the idea that one quantity (the input) completely determines another quantity (the output). This project is about special functions and orthogonal polynomials. A special function is a function which has appeared in the mathematical sciences so often that it has been given a name. Our research centers on a type of special function called a Green's function or a fundamental solution (named after the British mathematician George Green, who first developed the concept in the 1830s). Green's functions can be used to describe the influence of natural phenomena such as electromagnetism, gravity, heat and waves. For instance in electromagnetism, a Green's function describes the influence of a point charge. The possible inputs are all of space (with the exception of a singular region), and the output is the "force" exerted from the point charge throughout space.

Green's functions play an important role in the fundamental area of mathematics called partial differential equations, where they provide a powerful mechanism for obtaining their solutions. We study Green's functions in spaces which are curved, and in particular, those spaces which locally look the same in every direction. These spaces are called isotropic. Orthogonal polynomials and the special functions associated with them occupy a natural location in the study of these spaces. We focus on their behavior. Our study is fundamental to the mathematical understanding of the universe, which as Einstein showed is curved.

We have focused on the following.

1. The determination in separable coordinate systems of eigenfunctions and eigenfunction expansions for fundamental solutions of Laplace's equation on three-dimensional Euclidean space.

2. The computation of generalizations for generating functions of hypergeometric orthogonal polynomials, generalized series expansions for higher transcendental functions associated with linear sep- arable partial differential equations, and related definite integrals.

3. The determination of eigenfunction expansions and addition theorems connected with fundamental solutions for linear elliptic partial differential equaequations on isotropic Riemannian manifolds.

Hypergeometric orthogonal polynomials are polynomials which are orthogonal with respect to a measure on an interval of the real line. We study eigenfunction expansions of integration kernels in terms of orthogonal polynomials (i.e., Jacobi, Gegenbauer, Chebyshev, Legendre and Laguerre). Some important kernels which can be expanded in terms of these polynomials are fundamental solutions of linear partial differential equations in rotationally-invariant coordinate systems and generating functions.

In collaboration with Hans Volkmer (Department of Mathematical Sciences, University of Wisconsin Milwaukee) we have derived eigenfunction expansions for a fundamental solution of Laplace's equation on $\mathbf{R}^{3}$ in parabolic and elliptic cylinder coordinates [4]. These expansions are given in terms of Hermite functions, modified parabolic cylinder functions, Mathieu functions and modified Mathieu functions. As an application of orthogonality and closure relations, we have obtained new definite integrals for products of associated Legendre functions with Bessel functions, associated Legendre functions, Ferrers functions, and Chebyshev polynomials of the first kind [3]. Some of these new definite integrals correspond to infinite series addition theorems for fundamental solutions of elliptic PDEs and are obtained using orthogonality relations for Ferrers functions of the first kind with integer degree and order, and Chebyshev polynomials of the first kind. We also obtain new definite integrals from pre-existing definite integrals by the application of the closure relation for the Hankel transform.

In terms of real Euclidean space, we have used expansions of a power-law fundamental solution of the polyharmonic equation on $\mathbf{R}^{d}$ in terms of Chebyshev polynomials of the first kind and Gegenbauer polynomials to derive addition theorems in polyspherical coordinate systems for the resulting azimuthal Fourier coefficients. We have also developed polyspherical addition theorems for a logarithmic fundamental solution of the polyharmonic equation in even-dimensional Euclidean space by comparing azimuthal Fourier expansions of this fundamental solution to corresponding Gegenbauer polynomial expansions. 


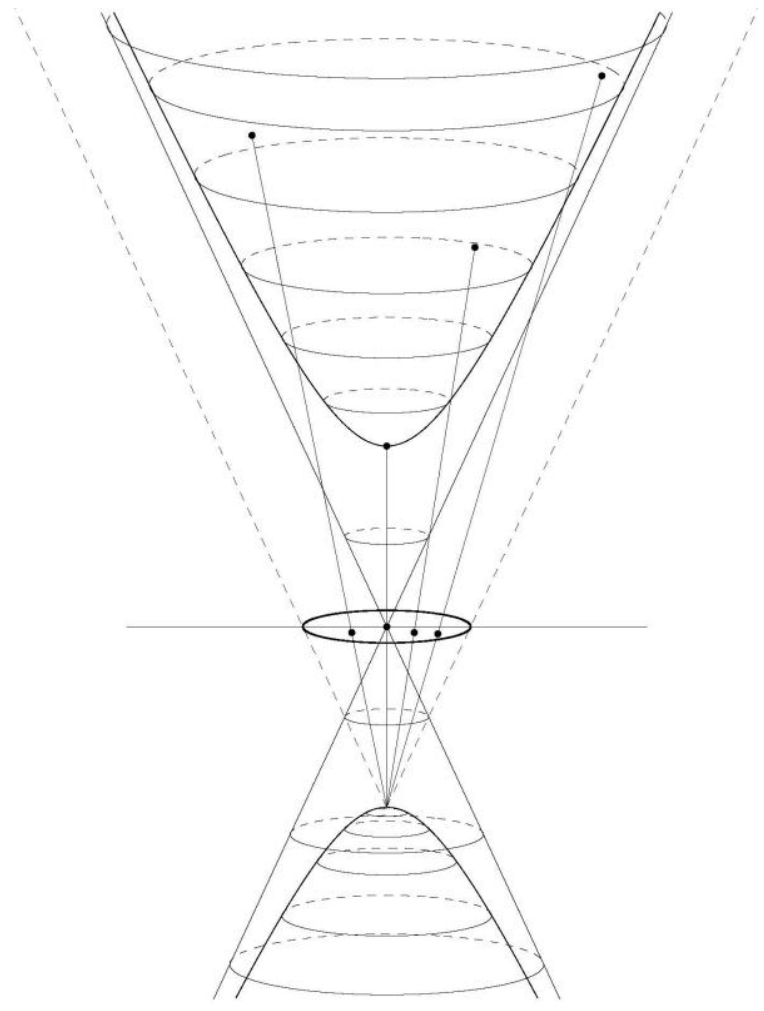

Figure 57. A graphical depiction of the hyperbolid model of hyperbolic geometry $\mathbf{H}_{R}^{d}$ with stereographic projection from the hyperbolid model to the Poincaré ball model.

We also study special function representations for fundamental solutions of Laplace's equation in terms of the global geodesic distance function on Riemannian spaces of constant curvature. In [1] we derive several alternative expressions for a fundamental solution of Laplace's equation $S_{R}^{d}$ on the $d$-dimensional $R$-radius hypersphere $\mathbf{S}_{R}^{d}$. These expressions include definite integral, Gauss hypergeometric function, finitesummation and Ferrers function of the second kind representations. In [2], we derive several alternative expressions for a fundamental solution of Laplace's equation $\mathcal{H}_{R}^{d}$ on the $d$-dimensional $R$-radius hyperboloid $\mathbf{H}_{R}^{d}$ (see Figure 57). These expressions include definite integral, Gauss hypergeometric function, finite-summation and associated Legendre function of the second kind representations.

Concerning eigenfunction expansions and addition theorems for fundamental solutions of linear elliptic PDEs on curved isotropic Riemannian manifolds, we have had recent successes in Cohl \& Kalnins (2012) [2] on $\mathbf{H}_{R}^{d}$. In [2], we compute azimuthal Fourier and Gegenbauer expansions for $\mathcal{H}_{R}^{d}$. In particular, we derive azimuthal Fourier expansions in rotationallyinvariant coordinate systems and Gegenbauer polyno- mial expansions in polyspherical coordinate systems which parameterize points on $\mathbf{H}_{R}^{d}$. The Gegenbauer polynomial expansion is given in terms of products of associated Legendre functions of the first and second kind and Gegenbauer polynomials which are the reproducing kernel for the space of polyspherical harmonics on a $(d-1)$-dimensional hypersphere embedded within $\mathbf{H}_{R}^{d}$.

On this project, we are currently collaborating with Gestur Ólafsson (Department of Mathematics, Louisiana State University). We have obtained for the rank one symmetric spaces, formulae for the global geodesic distance function as well as the radial coefficients which multiply the Laplace-Beltrami operator for the Grassmann submanifolds embedded within these symmetric spaces. A fundamental solution of Laplace's equation on the rank one symmetric spaces is radial and can be written as a solution to a second order ODE, of which eigenfunctions are known to be given in terms of specific Jacobi polynomials. Product addition theorems (in analogy with the addition theorem for Hyperspherical harmonics) which correspond to the harmonics on the embedded Grassmann submanifolds have also been computed. These are analogous to the polyspherical harmonics on embedded (d-1)dimensional hyperspheres within $\mathbf{H}_{R}^{d}$ and $\mathbf{S}_{R}^{d}$. One can expand the eigenfunctions of the Laplace-Beltrami operator on a $d$-dimensional rank one symmetric space in terms of the reproducing kernel of the harmonics on the $(d-1)$-dimensional embedded Grassmann manifolds $G^{d-1}$. We know that $L^{2}\left(G^{d-1}\right)=\oplus \mathcal{Y}_{m}$, where $\mathcal{Y}_{m}$ is the space of harmonics of degree $m$ on $G^{d-1}$. This is a finite dimensional Hilbert space where point evaluation is linear and continuous.

In [2], we computed azimuthal Fourier and Gegenbauer polynomial expansions for $\mathcal{H}_{R}^{d}$ along with corresponding polyspherical addition theorems. We aim to compute azimuthal Fourier, Gegenbauer polynomial expansions and polyspherical addition theorems for $S_{R}^{d}$ on $\mathbf{S}_{R}^{d}$. Our main goal is to understand the global analysis, derive and introduce eigenfunction expansions and generate corresponding special function addition theorems for a fundamental solution of Laplace's equation on the remaining rank one symmetric spaces such as complex, quaternionic and octonionic projective spaces as well as complex, quaternionic, and octonionic hyperbolic spaces.

[1] H. S. Cohl, Fundamental Solution of Laplace's Equation in Hyperspherical Geometry, Symmetry, Integrability and Geometry: Methods and Applications 7 (2011), 108 (14 pages).

[2] H. S. Cohl and E. G. Kalnins, Fourier and Gegenbauer Expansions for a Fundamental Solution of the Laplacian in the Hyperboloid Model of Hyperbolic Geometry, 
Journal of Physics A: Mathematical and Theoretical 45 (2012), 145206 (33 pages).

[3] H. S. Cohl and H. Volkmer, Definite Integrals using Orthogonality and Integral Transforms, Symmetry, Integrability and Geometry. Methods and Applications 8 (2012), 077.

[4] H. S. Cohl and H. Volkmer, Eigenfunction Expansions for a Fundamental Solution of Laplace's equation on $\mathbf{R}^{3}$ in Parabolic and Elliptic Cylinder Coordinates, Journal of Physics A: Mathematical and Theoretical 45 (2012), 355204 (20 pages). 


\section{Quantum Information}

An emerging discipline at the intersection of physics and computer science, quantum information science $(Q I S)$ is likely to revolutionize science and technology in the same way that lasers, electronics, and computers did in the $20^{\text {th }}$ century. By encoding information into quantum states of matter, one can, in theory, exploit the seemingly strange and counter-intuitive behavior of quantum systems to enable phenomenal increases in information storage and processing capability, as well as communication channels with extremely high levels of security. Although many of the necessary physical manipulations of quantum states have been demonstrated experimentally, scaling these up to enable fully capable quantum computers remains a grand challenge. We are engaged in (a) theoretical studies to understand the true power of quantum computing, $(b)$ collaborative efforts with the multi-laboratory experimental quantum science program at NIST to characterize and benchmark specific physical implementations of quantum information processing, and $(c)$ the development and assessment of technologies for quantum communication.

\section{Quantum Algorithms for Quantum Field Theories}

\author{
Stephen Jordan \\ Keith Lee (University of Pittsburgh) \\ John Preskill (Caltech)
}

\section{See feature article, page 17.}

\section{Quantum Computing Theory}

\author{
Scott Glancy \\ Emanuel Knill \\ Adam Meier \\ Mike Mullan \\ Yanbao Zhang \\ Bryan Eastin (Northrop Grumman) \\ Emilio Cobanera (University of Indiana) \\ Gerardo Ortiz (University of Indiana)
}

Our contributions to quantum computing and information theory at NIST span several application areas. We highlight our contributions to future improvements in the precision of atomic clocks, the analysis of experiments on the foundations of quantum mechanics, and the mathematics of physical dualities in condensed matter theory.

A promising strategy for improving the precision of atomic clocks is to exploit quantum correlations among multiple atoms to reduce measurement noise. In the relevant setting, an atomic clock consists of a classical "flywheel" that actually does the time keeping, and a fundamental frequency reference based on an atomic transition. The flywheel's frequency is not fundamental and must be corrected to match the frequency reference. The flywheel is very stable, but still drifts over time. Therefore, the flywheel's frequency must be continually controlled to match the frequency reference. Measurements of the frequency reference are used to correct the flywheel's frequency. To make the measurements as informative as possible, multiple atoms can be prepared in a quantum correlated state and, after an evolution period, measured in a general quantum basis. We adapted semidefinite programming techniques for optimizing quantum query algorithms to optimize the prepared state and final measurement for this purpose [1]. A key aspect of our method is to optimize a "cost function" that quantifies the quality of the measurement. Our original approach was greedy, optimizing an obvious quadratic cost independently of past measurements. We discovered that contrary to intuition, it is advantageous to make the cost function dependent on the past. To explore how much of an improvement can be obtained by exploiting quantum correlations, we implemented the first efficient simulation of an atomic clock that includes full Bayesian update cycles for correcting the flywheel frequency. Our first tests of the simulation confirmed what Till Rosenband (NIST PML) found in his explorations of measurement techniques on two and three atomic systems.

Our work includes collaborations on experiments that use entangled photons for fundamental tests of quantum mechanics. Recently, these experiments have been expanded with the goal of demonstrating deviceindependent random number generation (see page 79). The analysis of data from these experiments requires quantifying the evidence against conventional, local realistic explanations for experimental data. The quantification can be done by means of statistical pvalues in a way that is independent of the specifics of the experiment, thus enabling direct comparison of completely different experiments. Previously, we developed an asymptotically optimal method for computing such p-values for rejecting local realism. While this method is practical for experiments in progress, future experiments involve more complicated configurations requiring a more efficient approach. We 
have developed such an approach that is not limited by the complexity of the experimental configuration [2].

We continue to investigate techniques that promise to be useful for future fault-tolerant quantum computers. With B. Eastin, we completed work on a more efficient way of purifying so-called magic states needed for universal fault-tolerant quantum computing [3]. The work was particularly timely, as recent progress on IARPA's Quantum Computing Program suggests that many fault-tolerant quantum computations are dominated by the work needed for such purifications. Several research groups have already found use for our approach in their work. As part of our work on fault-tolerant architectures, we also refined our simulations of stabilizer codes, which will be an important part of the soon-to-be completed Ph.D. Thesis of Adam Meier.

Our work on physical dualities began with a now completed investigation of so-called non-Abelian dualities from the point of view of the framework of bond algebras developed by E. Cobanera and G. Ortiz [4]. These kinds of dualities can be used as important tools to analyze phase diagrams of classical and quantum thermodynamic systems defined on lattices. As part of our work, we sought novel examples of dualities that generalize those based on standard group theory and found a generalization to Hopf algebras in one dimension. Hopf algebras capture the essential algebraic properties of group algebras, so whenever an algebraic construction works for groups, one can attempt to define it more generally for Hopf algebras. In this case, the construction was successful. We are presently considering whether there are any physically (rather than mathematically) motivated examples in this class that cannot be obtained from a conventional group.

[1] M. Mullan, and E. Knill, Improving Quantum Clocks Via Semidefinite Programming, Quantum Informaton and Computation 12 (2012), 553-574.

[2] Y. Zhang, S. Glancy, and E. Knill, Asymptotically Optimal Data Analysis for Rejecting Local Realism, Physical Review A 84 (2011), 062118 (10 pages).

[3] A. Meier, B. Eastin, and E. Knill, Magic-State Distillation with the Four-Qubit Code, Quantum Information and Computation, to appear.

[4] E. Cobanera, G. Ortiz, and E. Knill, The Non-Abelian Duality Problem, in review.

\section{Quantum Computing Benchmarks}

\author{
Emanuel Knill \\ Adam Meier \\ Michael Mullan \\ Dietrich Leibfried (NIST PML) \\ Till Rosenband (NIST PML) \\ David Wineland (NIST PML)
}

Experimental efforts to demonstrate quantum devices suitable for quantum computations are rapidly growing and showing increasing success on multiple platforms. While the ion-trap platform is still leading the field, superconducting qubits are rapidly approaching parity, and solid-state qubits are showing increasing promise. One of our goals is to establish simple benchmarking protocols that can quantify the performance of these devices in a platform-independent way. Presently, our main benchmarking strategy involves the implementation of randomized quantum networks and comparing the output to that expected for an ideal network. This yields an "error per gate" that can be reported as a device quality measure. Our latest implementation of such a benchmark involved random two-qubit circuits and established an error per so-called Clifford operation as well as an error per phase-gate, one of the standard elementary two-qubit gates. This work appeared in Physical Review Letters [1] and established a method for benchmarking that can readily be scaled to more qubits. The technique has also been demonstrated in superconducting qubits by a group at IBM, obtaining similar results. In both cases, the experimental challenge is to improve the errors per gate by at least an order of magnitude so that it becomes conceivable to scale up the systems in a faulttolerant way.

While presently available quantum devices are unable to realize the promised computational advantages of quantum computers, they are still useful for demonstrating basic principles of quantum algorithms and may suggest new ways of improving measurement precision. An example application is the use of quantum algorithm techniques for improving the precision of atomic clocks, where the frequency standard is based on a clock transition that can also serve as a qubit. The quantum information perspective has already been used to advantage in the so-called $\mathrm{Al}^{+}$-based quantum logic clock of the NIST Ion Storage group. Our theoretical work on quantum-algorithmbased clock optimization may be directly applicable to this clock. D. Leibfried recently suggested that the principles could be demonstrated on the more controllable $\mathrm{Be}^{+}$or $\mathrm{Mg}^{+}$quantum bits being developed for quantum information processing. This would also serve as a benchmark for algorithmic controllability of these quantum bits. We are in the process of determining the feasibility of this suggestion. 
[1] J. P. Gaebler, A. M. Meier, T. R. Tan, R. Bowler, Y. Lin, D. Hanneke, J. D. Jost, J. P. Home, E. Knill, D. Leibfried, and D. J. Wineland, Randomized Benchmarking of Multiqubit Gates, Physical Review Letters 108 (2012), 260503 (5 pages).

\section{Quantum State Estimation Theory and Applications}

\author{
Scott Glancy \\ Emanuel Knill \\ Katelyn Weber (Montana State University) \\ Mark Girard (Freiburg Institute for Advanced Studies) \\ Gene Hilton (NIST PML) \\ Kent Irwin (NIST PML) \\ Konrad Lehnert (NIST PML) \\ Will Kindel (University of Colorado) \\ Hsiang S. Ku (University of Colorado)
}

Many emerging technologies will exploit quantum mechanical effects to enhance metrology, computation, and communication. Developing these technologies requires improved methods to measure the state of quantum systems. Quantum state estimation is a statistical problem of estimating an underlying quantum state using a collection of measurements made on independently prepared copies of the state. Accurate quantum state estimation allows experimentalists to answer the questions "What quantum state does my device prepare?" and "How confident am I that it has prepared that state?"

One popular method to estimate quantum states is the maximum likelihood method, according to which the reported estimate is the state that maximizes the likelihood for the observed data. Finding the maximum likelihood state requires an iterative numerical optimization procedure. We formulated a statistically relevant criterion for stopping the iterations when the likelihood is "large enough", depending on the proposed use of the estimated state [2]. We used classical statistical theory to describe several likelihood ratio confidence regions for the state estimate and gave stopping rules in each case. This work has provided the first rigorous stopping criteria for maximum likelihood quantum state tomography, allowing researchers to calculate good quantum state estimates without wasting computational resources. The confidence regions we described can be used to give uncertainties for quantum state estimates.

Motivated by shortcomings in the maximum likelihood method (especially estimator bias and computation time) we implemented a new method for quantum state characterization based on sampling of "pattern functions". A quantum state's expectation value of an observable (such as fidelity with any other pure state) can be expressed as the expectation value of a pattern function, where the pattern function is a function of experimentally measurable quantities. By repeating the measurement and calculating the mean value of the pattern function evaluated for each measurement result, one can estimate the desired expectation value and its uncertainty. We found that the pattern function method avoids the bias that plagues maximum likelihood quantum state tomography, requires far less computational effort, and provides rigorous uncertainty estimation. However, given equal data sets, the pattern functions showed slightly larger statistical error.

We continue our collaboration with the JILA laboratory of Konrad Lehnert through the DARPA project titled "Analog Quantum Integrated Circuits". The goal of this project is to create entangled states in superconducting microwave circuits and to use that entanglement for quantum teleportation [1]. In 2012 the laboratory prepared an entangled state of quantum microwaves and verified the entanglement with preliminary measurements. We are developing methods to calibrate the measurements and fully quantify the amount of entanglement.

[1] S. Glancy, W. Kindel, H. S. Ku, E. Knill, K. D. Irwin, G. C. Hilton, L. R. Vale, and K. W. Lehnert, Progress Towards Quantum Teleportation of Traveling Microwaves, Workshop-School in Quantum Computation and Information, Fortaleza, Brazil, October 8, 2012.

[2] S. Glancy, E. Knill, and M. Girard, Gradient-based Stopping Rules for Maximum-likelihood Quantum-state Tomography, New Journal of Physics 14 (2012), 095017.

\section{Quantum State Tomography using Compressed Sensing}

\author{
Yi-Kai Liu \\ Steven T. Flammia (University of Sydney) \\ David Gross (University of Freiburg) \\ Jens Eisert (Freie Universität Berlin)
}

Quantum state tomography is a family of techniques for estimating the state of a quantum system. Tomography provides a full characterization of the state, including all effects due to noise and decoherence. This makes it a useful tool in experiments involving quantum-enhanced metrology and quantum computation. However, tomography of large quantum systems is very resource-intensive: the required number of state preparations and measurements grows exponentially with the number of qubits.

Previously we studied methods for quantum state tomography based on compressed sensing, which can reconstruct quantum states that are approximately low- 
rank, while achieving a quadratic reduction in the number of measurement settings. We have proved tight error bounds, using a technique based on the restricted isometry property (RIP). In addition, in the special case of pure states, we have proved that compressed tomography has nearly optimal sample complexity (among all methods using two-outcome Pauli measurements).

In the past year, we have generalized our results on sample complexity to apply to low-rank mixed states; again, we find that compressed tomography is nearly optimal. We have also developed some useful tools: a more general version of our direct fidelity estimation technique, which can be used to certify low-rank mixed states; and a very simple compressed sensing technique for quantum process tomography.

We have run numerical simulations, which show that the sample complexity of compressed tomography is largely insensitive to the number of Pauli observables that one measures. This gives rise to a new degree of freedom when designing experiments: one can either measure a small set of observables relatively accurately, or measure a large (e.g., complete) set of observables relatively coarsely. Finally, our simulations demonstrate that compressed tomography is more accurate than standard maximum-likelihood estimation (MLE) in a variety of situations, given comparable experimental resources.

We are continuing to investigate compressed tomography of continuous-variable systems, as well as practical uses of compressed tomography in ion trap quantum information processors.

[1] S. T. Flammia, D. Gross, Y.-K. Liu and J. Eisert, Quantum Tomography via Compressed Sensing: Error Bounds, Sample Complexity, and Efficient Estimators, New Journal of Physics 14 (2012), 095022.

[2] M. Ohliger, V. Nesme, D. Gross, Y.-K. Liu and J. Eisert, Continuous-variable quantum compressed sensing, in review.

\section{Cryptography Beyond Quantum Key Distribution}

\section{Yi-Kai Liu}

Since Bennett and Brassard's discovery of quantum key distribution in 1984, many researchers have wondered whether quantum information can be used to perform other cryptographic tasks with informationtheoretic security. Unfortunately, there are strong theoretical results that rule out the possibility of quantum bit-commitment and most other kinds of secure computation. Interestingly, however, some of these cryptographic tasks become possible, if one assumes additional restrictions on the adversary's power; for instance, one may assume that the adversary can only perform k-local measurements, or that he has bounded or noisy storage.

We have been studying a model where it is possible to build isolated qubits, which can be measured individually, but cannot be entangled. This is motivated by recent experimental work with nitrogen vacancy centers, and possible cryptographic applications such as one-time memories and one-time programs.

\section{Post-Quantum Cryptography}

\author{
Stephen Jordan \\ Yi-Kai Liu \\ Lily Chen (ITL CSD) \\ Dustin Moody (ITL CSD) \\ Rene Peralta (ITL CSD) \\ Ray Perlner (ITL CSD) \\ Daniel Smith (ITL CSD)
}

Quantum information science creates both new challenges and new opportunities for cryptography. Peter Shor's 1994 discovery of efficient quantum algorithms for integer factorization and discrete logarithms showed that currently used cryptosystems (such as RSA, Diffie-Hellman and elliptic curve cryptosystems) will not remain secure in a world with quantum computers. The search for alternative cryptosystems secure against quantum attack has grown into an active subfield called post-quantum cryptography. Staff members of the ACMD, in collaboration with the ITL Computer Security Division, are currently studying various aspects of post-quantum cryptography.

Currently there are three main classes of cryptosystems considered to be promising post-quantum candidates: lattice-based (such as NTRU), code-based (such as McEliece), and multivariate (such as HFE and its variants). Significant open questions remain about each of these candidates. First, straightforward implementations of lattice-based and code-based cryptosystems suffer from large key sizes, scaling quadratically with the underlying security parameter. By incorporating additional structure into the keys, one can reduce their size; however, the security of these structured keys against classical or quantum attack remains an area of active research. In addition, recent successful attacks on multivariate cryptosystems have raised questions about what kinds of algebraic constructions suffice to ensure the security of these schemes. Finally, while there are some proposals for post-quantum encryption schemes that achieve key and message sizes comparable to RSA, there continue to be challenges in developing post-quantum signature schemes that are equally efficient. Thus there has been some serious consideration of alternative solutions, such as hash-based signatures. 
In the past year we have surveyed various postquantum cryptosystems, to get an overview of the different constructions, security proofs, and cryptanalytic attacks. In addition, we have surveyed recent work on quantum algorithms and experimental implementations of quantum computers. Overall, it appears that there are promising candidates for post-quantum cryptography, although many of these are still at the basic research stage. The next step will be to perform a detailed evaluation of these candidate cryptosystems, to move them closer to practical use.

\section{Quantum and Classical Circuit Obfuscation with Braids}

\author{
Stephen Jordan \\ Gorjan Alagic (Caltech) \\ Stacey Jeffery (University of Waterloo)
}

A circuit obfuscator is an algorithm that translates logic circuits into functionally-equivalent similarly-sized logic circuits that are hard to understand. While ad hoc obfuscators exist, theoretical progress has mainly been limited to no-go results. In this project we propose a new notion of circuit obfuscation, which we call partial indistinguishability. We then prove that, in contrast to previous definitions of obfuscation, partial indistinguishability obfuscation can be achieved by a polynomial-time algorithm. Variants of this algorithm can be applied to both classical and quantum circuits.

Circuit obfuscation, while an intuitively natural concept, is difficult to define formally. In 2007, Goldwasser and Rothblum made substantial progress by defining indistinguishability obfuscation. An indistinguishability obfuscator $O$ is a map from circuits to circuits, such that if $A$ and $B$ are functionally equivalent then $O(A)$ and $O(B)$ are indistinguishable. Intuitively, such an obfuscator hides all information about the implementation of $A$ and $B$ (though not necessarily their functionality).

If $O$ is a fixed, deterministic map then for $O(A)$ to be indistinguishable from $O(B)$, one requires $O(A)=O(B)$. However, $O$ may also be a probabilistic map, in which case it suffices for the probability distribution $O(A)$ to be computationally infeasible to distinguish from the probability distribution $O(B)$. However, complexity-theoretic arguments show that a polynomial-time deterministic indistinguishability obfuscator for general circuits cannot exist unless $\mathrm{P}=\mathrm{NP}$. Even in the probabilistic case, no efficient construction for indistinguishability obfuscation of general circuits is known.

In this work, we propose a weaker definition, which we call partial-indistinguishability obfuscation. Here, given equivalent circuits $A$ and $B$, we demand
$O(A)=O(B)$ only if $A$ can be obtained from $B$ by some sequence of logic-gate identities taken from a fixed finite set $G$. If $G$ is a complete set of gate identities then one recovers Goldwasser and Rothblum's notion of indistinguishability obfuscation. For smaller sets of gate identities, this defines a weaker notion of obfuscation, but on the other hand, it may be actually achievable in polynomial time. Specifically, using ideas from topological quantum computation, we show that efficient obfuscators exist for both classical quantum circuits when $G$ is taken to be the relations of the Artin generators for the braid group.

[1] G. Alagic, S. Jeffery, and S. P. Jordan. Partialindistinguishability obfuscation using braids, in preparation.

\section{Generating Random Bits from Entangled Quantum States}

\author{
Scott Glancy \\ Stephen Jordan \\ Manny Knill \\ Yi-Kai Liu \\ Paulina Kuo \\ Alan Mink \\ Xiao Tang \\ Lawrence Bassham (ITL CSD) \\ Michaela Iorga (ITL CSD) \\ Andrew Rukhin (ITL SED) \\ Joshua Bienfang (NIST PML) \\ Alan Migdall (NIST PML) \\ Sae Woo Nam (NIST PML)
}

Quantum information technology enables new cryptographic schemes whose security is based on physical, rather than computational, assumptions. This year, staff members of the ACMD, as well as of other divisions of the ITL and the PML, have teamed up to initiate a NIST Innovations in Measurement Science project to develop a random number generator based on quantum entanglement. Such a generator could be used to generate the private keys used in standard cryptographic schemes. In addition, the team plans to integrate the quantum random number generator with the Computer Security Division's random number beacon. The beacon will publish time-stamped random numbers on the internet, which can then be used to implement various secure multiparty protocols, such as privacy-preserving auctions and selective disclosure of personal information.

At the heart of this project is a fundamental connection between quantum nonlocality and randomness. This is illustrated by "Bell tests," which are experiments using entangled pairs of photons, whose results cannot be predicted by any classical ("local realistic") 
model, but are in agreement with quantum mechanics. The result of a Bell test turns out to have a surprising property: it is guaranteed to be random, in the sense that it could not have been predicted beforehand. Moreover, this guarantee can be made "deviceindependent," i.e., any Bell test that passes a certain verification procedure, which involves its input-output behavior but not its internal functioning, can be guaranteed to produce a random output.

Building a device-independent secure random number generator poses many experimental and theoretical challenges. In particular, it requires the construction of a "loophole-free" Bell test. Achieving such a test requires very high efficiency generation, transmission, and detection of entangled photons, which only recently has appeared within reach of experimental technique. In addition, there are theoretical challenges in parlaying such a test into a useful cryptographic resource, as one must show that the scheme is secure against both the subtleties of nature and the attacks of a malicious adversary.

\section{Quantum Communications}

\author{
Xiao Tang \\ Oliver Slattery \\ Paulina Kuo \\ Yong-Su Kim \\ Barry Hershman
}

We conduct experimental research in quantum communications to identify the potential for and limitations of this technology for the IT sector, and to develop the requisite measurement science to support commercialization. More specifically, our research agenda includes single photon creation, transmission, storage, transduction and detection. Our goal is to demonstrate a complete quantum communications system that will create strategically selected single photons, transmit them (flying qubits) and interface them with an atomic quantum memory (stationary Qubit) for storage and processing.

Previously, our research group developed an efficient frequency up-conversion detector for single photon level light at $1.31 \mu \mathrm{m}$ by pumping them at 1.55 $\mu \mathrm{m}$. We then worked with Stanford University to develop an up-conversion detector targeting $1.55 \mu \mathrm{m}$, the other low-loss transmission window in silica based fibers. In this case, the single photons at $1.55 \mu \mathrm{m}$ are pumped at $1.9 \mu \mathrm{m}$ creating near visible single photons efficiently detectable using a silicon-based avalanche photo diode (APD). The $1.55 \mu \mathrm{m}$ system achieves a detection efficiency of more than $35 \%$ with low noise characteristics [1]. This development extended upcon- version detection wavelength from near $1.3 \mu \mathrm{m}$ to 1.55 $\mu \mathrm{m}$.

One of the drawbacks of silicon based avalanche detectors is so-called detection jitter, which limits the maximum detection rate of the system. To overcome this, our team previously demonstrated a proof-ofprinciple scheme to increase the data rate of quantum communication systems equipped with up-conversion detectors [2]. During this fiscal year, NIST and Stanford researchers developed a unique dual-channel upconversion device [3] in which signal photons at 1.3 $\mu \mathrm{m}$ are pumped by two slightly separated wavelengths near $1.55 \mu \mathrm{m}$ and converted to two photons at slightly separated visible wavelengths in the two channels. The visible photons are separated using a dispersion element and sent to two distinct silicon based APDs for detection. Therefore, the system data rate can be doubled with high detection efficiency.

The team has further developed our signal photon sources to include narrowed single photons at $895 \mathrm{~nm}$ (compatible with atomic transitions of Cesium) and $1310 \mathrm{~nm}$ (suitable for transmission over long distances). In addition, the development of a Cesium based quantum memory system was started during this yearincluding outfitting a dedicated laboratory toward that development.

The team also successfully completed an SBIR project to commercialize the upconversion detector technology developed at NIST.

More details of the development and research progress by the team in recent years can be found in the newly published article in Physics Reports [4].

[1] J. S. Pelc, L. Ma, C. R. Phillips, Q. Zhang, C. Langrock, O. Slattery, X. Tang, and M. M. Fejer, Longwavelength-pumped upconversion single-photon detector at $1550 \mathrm{~nm}$ : performance and noise analysis, Optics Express 19:22 (2011) 21445.

[2] L. Ma, J. C. Bienfang, O. Slattery and X. Tang, Upconversion single-photon detector using multiwavelength sampling techniques, Optics Express 19:6 (2011) 5470.

[3] J. S. Pelc, P. S. Kuo, O. Slattery, L. Ma, X. Tang, and M. M. Fejer, Dual-channel, single-photon upconversion detector at 1.3 $\mu \mathrm{m}$, Optics Express 20:17 (2012), 19075.

[4] L. Ma, O. Slattery, X. Tang, Single photon frequency up-conversion and its applications, Physics Reports (2012). 


\section{Low Density Parity Codes for Quantum Key Distribution}

Alan Mink

Anastase Nakassis (ITL ANTD)

Quantum information science incorporates quantum mechanics into information technology. It is an emerging field thought to have the potential to cause revolutionary advances. One promising early area is quantum key distribution (QKD), which is a protocol that uses a pair of unsecured communication channels, one quantum and one classical, to establish a shared secret between two parties, Alice and Bob. This shared secret is then used to encrypt messages between Bob and Alice. QKD has been proven information theoretically secure, unlike classical key distribution, which relies on computational complexity.

There are four stages to the QKD protocol. Stage 3 is where Alice and Bob exchange information over the classical channel to correct errors between their bit sequences (the shared secret) without exposing the value of their bits. There is a large body of information about error correction codes, but QKD presents a somewhat different environment than conventional communication. Expected QKD error rates are higher ( $1 \%$ to $11 \%$ vs. $0.1 \%$ or below), error correction information is sent separately over an error free channel, the amount of information that the error correction code leaks about the data must be kept below a given limit, and data retransmission is not possible.

The original QKD error correction algorithm, Cascade, requires multiple round trip communications. Each trip leaks additional information, and the number of trips required is unknown in advance. An alternative error correction method, called low density parity check (LDPC), has become popular for QKD, because it only requires a single roundtrip communication, and therefore the amount of leaked information is known in advance. Also, a single trip reduces communication delays, a benefit as the distance between Alice and Bob increases.

We have investigated LDPC error correction codes customized for QKD. In particular, we have developed tighter bounds for the information leaked during QKD LDPC [1]. We have also developed efficient LDPC software and hardware implementation techniques.

We then followed up with a description of the LDPC forward error correction algorithm adapted for the QKD protocol in a form that is readily applied by developers [2]. A sparse matrix describing a set of check sums is a key component of LDPC. Our description includes sources for the LDPC matrices as well as lookup tables, data structures, and a pseudo code description of the complete LDPC algorithm, both of which are normally absent from LDPC descriptions. We also evaluated these matrices for their QKD correction performance, since different matrices present a tradeoff between correction performance and information leakage. A matrix that can correct a larger error rate also leaks more information, directly reducing the amount of secure key, possibly to zero. We also discussed the computational precision required for this LPDC algorithm and showed a simple heuristic technique to boost performance with less precision.

The objective of the QKD 3rd stage is to perform error correction such that it will optimize the amount of secure key in the time allotted. Thus, we are pursuing faster implementations, using hardware and GPUs, as well as devising strategies to maximize overall secure key production, within the time constraints available.

[1] A. Nakassis and A. Mink, LDPC Error Correction in the Context of Quantum Key Distribution, in Proceedings of the SPIE: Defense Security \& Sensing, Baltimore, MD, April 2012.

[2] A. Mink and A. Nakassis, LDPC for QKD Reconciliation, The Computing Science and Technology International Journal 2:2 (2012), 2162-0687. 


\section{Foundations of Measurement Science for Information Systems}

Modern information systems are astounding in their complexity. Software applications are built from thousands of interacting components. Computer networks interconnect millions of independently operating nodes. Large-scale networked applications provide the basis for services of national scope, such as financial transactions and electrical power distribution. In spite of our increasing reliance on such systems, our ability to build far outpaces our ability to secure. Protocols controlling the behavior of individual nodes lead to unexpected macroscopic behavior. Local power anomalies propagate in unexpected ways, leading to large-scale outages. Computer system vulnerabilities are exploited in viral attacks, resulting in widespread loss of data and system availability. The long term stability of our critical infrastructure is simply unknown. Measurement science has long provided a basis for the understanding and control of physical systems. Similar types of deep understanding and insight are lacking for complex information systems. We seek to develop the mathematical foundations needed for the emergence of a true measurement science for complex networked information systems.

\section{A Game-theoretic Approach to the Security of Complex Systems}

Assane Gueye

Vladimir Marbukh

Ion Matei (NIST EL)

John S. Baras (University of Maryland)

Richard La (University of Maryland)

Aron Laszka, (Budapest Univ. of Tech. and Economics)

Today, almost all critical infrastructures of national importance-transportation, financial, power grids, military, intelligence systems, and health and safety systems - rely on information and communication technologies (ICT). Despite this vital dependency on ICT systems, our fundamental knowledge about their global behavior is quite primitive: today there is no science that offers the knowledge needed to build globally predictable systems. Moreover, recent failures of critical infrastructures (e.g., communication and financial networks, power grids) have exposed troubling deficiencies in our ability to prevent and mitigate such catastrophic events. Metrics are needed which can be used for effectively assessing a system's level of security and reliability. Unfortunately, conventional metrics are based on unrealistic assumptions, e.g., stand-alone systems, steady state, lack of selfish user behavior in general and of malicious behavior in particular. Finally, we still lack a good understanding of the fundamental limits in our ability to design large systems that are secure and resilient. Only such understanding will enable the development of realistic and cost-effective tools to deal with present and future security threats.

Our research effort strives to address the aforementioned issues from a first principle/science-based approach. We are exploring three closely related and interdependent research directions: (a) characterizing macro-scale structures and dynamics of large-scale interconnected systems, (b) identifying fundamental metrics that are indicators of the system's inherent lev- el of security and reliability, and (c) using these metrics to develop efficient tools for cybersecurity risk management and appropriate feedback loops to mitigate risks. We envision enhancing conventional models and proposing new ones by explicitly recognizing that ICT infrastructures are distributed large-scale systems where some components (e.g., users, processes) are selfish and other components are malicious. We plan to leverage recent advances in complex systems research, and game-theoretic models of selfish and malicious behavior, as well as our growing ability to collect and analyze large amounts of data. We will also build upon classical analytical approaches such as decision theory, risk theory, information theory, and control theory. To reach this interdisciplinary goal, we envision close collaboration with NIST staff members as well as faculty members at the University of Maryland.

Our research in the last year has demonstrated the important role game theory can play in studying security problems by capturing the strategic nature of adversaries. In particular, by modeling some idealized security scenarios as games between an attacker and a defender, we have shown that the ability of a malicious/selfish agent to acquire and exploit system information may alter conventional predictive metrics of system behavior. The next step is to bring current models closer to real-life applications, validate them against real-life data, and propose new and improved models. To this end, we have recently consolidated our earlier work and built upon it to propose new metrics for quantifying network vulnerability to attacks [1]. Using these metrics, we have proposed and discussed a framework for analyzing the risk/cost tradeoff for supply-demand networks [2]. In [3] the framework was generalized to other networks and the complexity of deriving the risk/cost tradeoff curve was analyzed. Finally, we have established the relationship between the proposed vulnerability metrics and well-known graph theory notions [4]. 
In the next year, we plan to utilize these metrics for the analysis of ICT systems. These systems are complex interconnected networks for which the local (micro) interactions of agents (some selfish, others malicious) can lead to the emergence of global (macro), often undesirable behaviors. For such large systems, we envision developing tools and models that can help us understand the underlying process that leads to "phase transitions" in the system's global behavior. This can be used to design systems so that their behavior can be predicted prior to building them, as well as during operation. For such networks of interacting agents, the notion of trust plays a central role in the process of local decision making. We intend to study how trust between the agents and the presence of both positive and negative externalities influence the dynamics and global behavior of such systems. Understanding the system's behavior will enable the development of effective control methods to mitigate the risk of catastrophic events.

The human factor is another aspect of the security process that we would like to explore. We are particularly interested in the issues of incentive and usability: how to develop the right mix of incentives (policies, laws) to promote adoption of best practices in cybersecurity, and how to develop security solutions that are intuitive and user-friendly enough to permit easy adoption. Finally, while our research is focused on the security of ICT systems, we plan to extend and apply the results to other critical systems such the power grid, water distribution, and transportation systems.

[1] A. Gueye, V. Marbukh, and J. C. Walrand, Towards a Quantification of Communication Network Vulnerability to Attacks: A Game Theoretic Approach, in Proceedings of the 3 rd International ICST Conference on Game Theory for Networks, Vancouver, Canada, May 2012.

[2] A. Gueye and V. Marbukh, A Game-Theoretic Framework for Network Security Vulnerability Assessment and Mitigation, in Proceedings of the 3rd Conference on Decision and Game Theory for Security (GameSec), Springer-Verlag, Berlin, 2012.

[3] A. Lazska and A. Gueye, Quantifying Network Topology Robustness under Budget Constraints, in review.

[4] A. Gueye, A. Lazska, J. Walrand, and V. Anantharam, A Polyhedral-Based Analysis of Nash Equilibrium of Quasi-Zero-Sum Games and its Applications to Communication Network Security, in review.

\section{An Algebraic Formulation for the Analysis and Visualization of Network Graphs}

\author{
Roldan Pozo
}

Characterizing the topological structure of large graphs is an important problem in network science. While it is straightforward to visualize small networks of hundreds or a few thousands of vertices and edges using conventional graph visualization packages, attempting to render large networks (unless they exhibit a special structure, such as a tree-like topology) is nearly impossible. In such cases the rendered image is nearly a solid blot of color in which is difficult to see any meaningful information. This difficulty is largely due to (1) the presence of high-degree nodes (hubs) which entangle many parts of the graph with itself, and (2) the sheer number of vertices and edges (well into the millions and billions) which are becoming common in network science.

An alternate approach is to visualize important network properties, rather than the actual network itself. Such network portraits attempt to capture interesting attributes of a large graph, such as degree distribution, or its connectivity properties. In this research area, we focus on a particular type of information: the distribution of "fringe" communities: small connected components of a graph, in the absence of high-degree hubs. In a social science context, for example, they represent independent or rogue groups, highly connected amongst themselves, but often lost with the inclusion of more popular nodes.

The Q-matrix, in its fundamental formulation, is a connected component size distribution matrix for a percolation process on a network graph. As a simple example, consider the component size distribution of degree-limited subgraphs on an undirected network. Figure 59, for example, displays the non-trivial connected components of a subgraph of the math.nist.gov website containing only nodes of (in+out) degree 25 or less. The image reveals various structures, e.g., ladders, stars, and cluster patterns representing tightly coupled webpages. These form the nucleus of fringe communities of web pages, and by varying the degree parameter we can direct the size and granularity of groups revealed. As the degree threshold is increased from 1 to the maximum degree of the original graph, we can form a connected component size distribution, which can be encoded as a row of a (sparse) matrix. Thus, the Q-matrix formulation is $Q(i, j)=$ number of connected components of size $j$ in a subgraph where the maximum degree is equal to or less than $i$. This matrix is too large to view directly, but one may render its image by projecting the nonzero values onto the $z$-axis and displaying it as a three- 


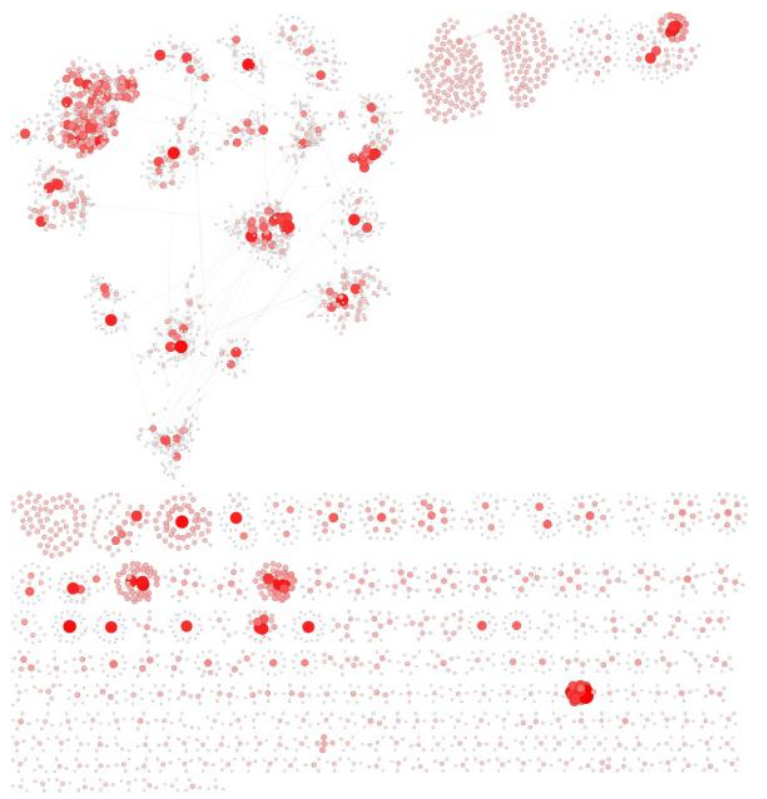

Figure 59. The non-trivial connected components of a subgraph of the math.nist.gov website containing only nodes of (in+out) degree 25 or less.

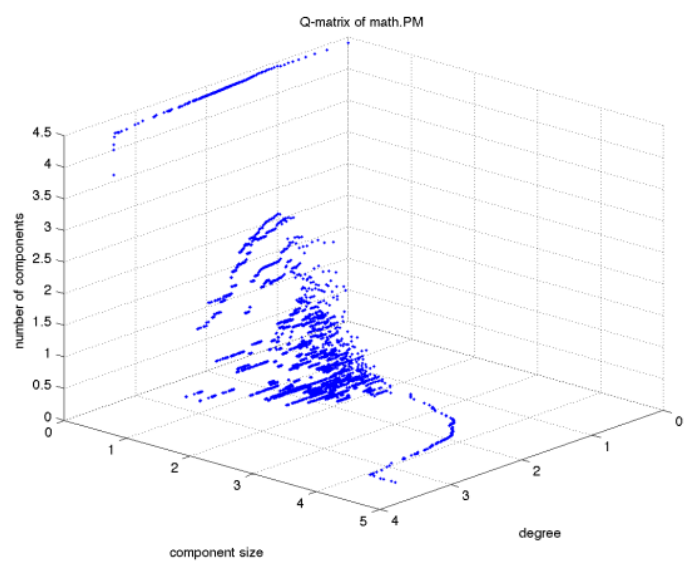

Figure 60. The Q-matrix diagram of the math.nist.gov website.

dimensional structure, as show in Figure 60. The Qmatrix of a graph can be considered as a generalization of its degree-distribution. However, it encodes other properties, such as the formation and growth of its giant component, its connected subparts, as well as its size parameters. In fact, common network metrics (degree distribution, number of connected components, number of vertices and edges) can be extracted from the Q-matrix using simple linear algebra operations.

Among the interesting characteristics of the Qmatrix formulation is that network graphs from similar application areas (e.g., social networks, email networks, web graphs, peer-to-peer networks, road networks, citation graphs) share similar visual characteristics. This makes such a framework useful for network identification and classification. Indeed, the
Q-matrix can be used to generate meaningful thumbnail images for network data collections.

Another useful feature is that a Q-matrix (and its plot) is unique to each network graph. That is, unlike general graph layout schemes used in visualizations, there is only one Q-matrix visualization. This is in contrast to conventional visualization and layout methods, in which the same graph may be rendered in multiple configurations. Finally, the computation of the Q-matrix is highly efficient - graphs with millions of vertices and edges can be processed in seconds on common desktop computers [1].

[1] R. Pozo, Efficient Q-matrix Computation for the Visualization of Complex Networks, in Proceedings of the 8th International Conference on Signal Image Technology \& Internet Based Systems, 2012.

\section{Threshold Digraphs}

\section{Brian Cloteaux \\ Elizabeth Moseman \\ James Shook \\ M. Drew LaMar (College of William \& Mary)}

A typical problem from the random modeling of networks is generating a graph with a given degree sequence. A threshold sequence is a graphical sequence that can only be realized by one labeled graph. The unique graph produced by these sequences is called a threshold graph (see Figure 58). These sequences define the boundary between graphical and non-graphical sequences, and understanding them is vital for the creation of algorithms for manipulating and realizing degree sequences. While a great deal of theory has been developed for undirected threshold sequences [2], there are few published results for directed threshold sequences.

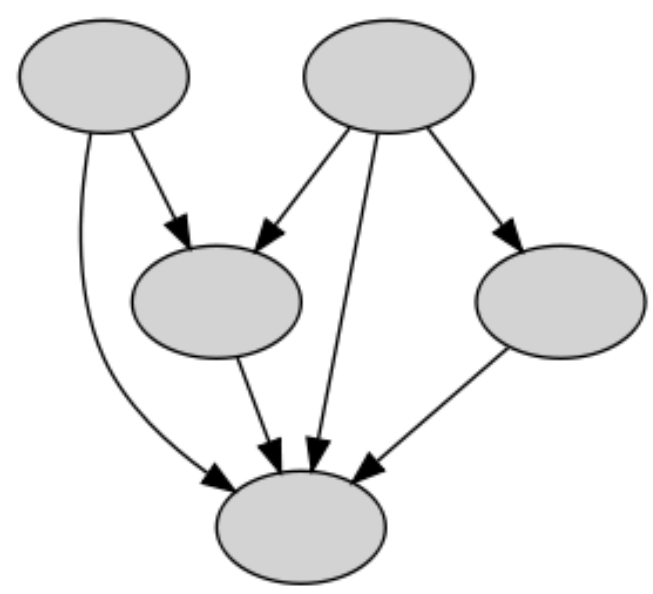

Figure 58. Example of a Threshold Digraph 
In order to create better network models, we have developed a number of new fundamental results concerning threshold digraphs. This project has enabled us to condense many of the known results in this area, while also developing several new results to describe these sequences. This has enabled us to create a new algorithm for the problem of creating a general realization of a digraph from a degree sequence. In addition, this work has produced an extremely short proof of the classical theorem for testing if a degree sequence if graphical, known as the Fulkerson-Chen inequalities. Further, we have shown an extension of the Fulkerson-Chen inequalities where the conditions necessary for them to be applied can be relaxed.

Beyond the immediate benefits of this project, such as the new algorithm for generating realizations, these results have application for creating new and faster algorithms in areas such as random network model generation (see below) and degree sequence manipulation. In addition, this work has led to a better understanding of how real-world networks contrast to these extremal examples of digraphs.

[1] B. Cloteaux, M. D. LaMar, E. Moseman, and J. Shook, Threshold Digraphs, arXiv:1212.1149, in review.

[2] N. V. R. Mahadev and U. N. Peled, Threshold Graphs and Related Topics, Elsevier, 1995.

\section{Random Generation of Digraphs}

\section{Elizabeth Moseman}

James Shook

Continuing our work in [2] on directed networks (digraphs), we address the problem of generating a model from a known degree sequence. Studying these models is the first step in understanding how the degree sequence constraint affects the network. A basic observation is that a directed network can be represented by a zero-one matrix with zero trace and the row and column sums representing the network degree sequence. There are algorithms in $[1,3,4]$ to generate models in both contexts. The known algorithms either have very large running time [3] or do not generate the samples uniformly $[1,4]$.

We have developed an algorithm to generate directed graphs with a given degree sequence that is significantly faster than known algorithms. Our algorithm runs in $O(m n)$ time where $m$ is the number of ones in the matrix and $n$ is the number of columns of the matrix. The method uses sequential importance sampling to generate models. The advantage of our algorithm is that we can calculate the number of ways to generate a given realization and the probability. Similar to [3], we measure the efficiency of our algorithm using the coefficient of variation $\left(c v^{2}\right)$. The closer $c v^{2}$

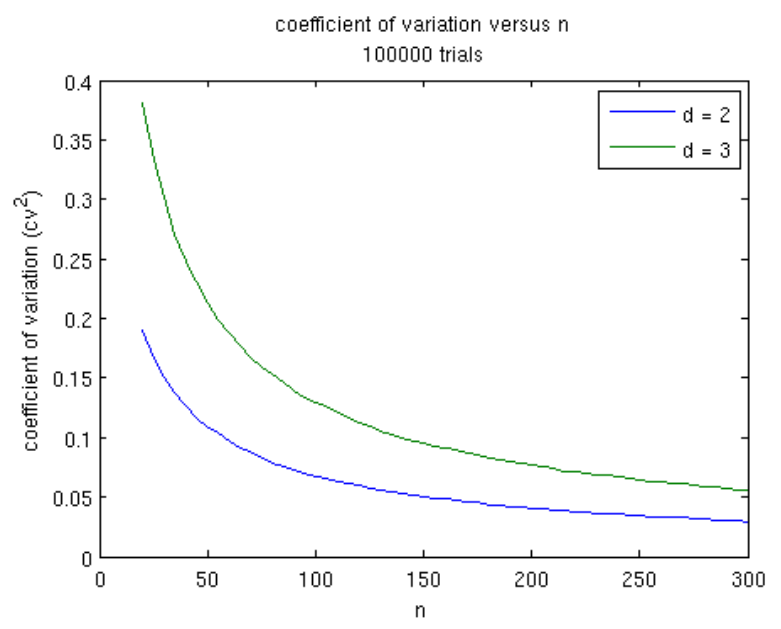

Figure 61. Coefficient of variation in 2 and 3 regular graphs.

is to zero, the closer the generation of random digraphs is to uniform. We tested our algorithm on constant degree sequences. In Figure 61 the $c v^{2}$ is plotted as a function of the number of nodes $(n)$ after 100,000 trials. The data suggests that the algorithm gets closer to uniform as $n$ increases. We are still testing our algorithm on a variety of different degree sequences.

In addition to modeling digraphs, our algorithm is able to estimate the number of digraphs with the specified degree sequence. A method of generating random models and an estimate of the number of digraphs leads to a better understanding of how network observables behave on these networks. In turn, this leads to a better understanding of how real-world networks differ from the models that we can generate.

[1] Y. Chen, P. Diaconis, S. P. Holmes, and J. S. Liu, Sequential Monte Carlo Methods for Statistical Analysis of Tables, Journal of the American Statistical Association 100 (2005), 109-120.

[2] B. Cloteaux, M. D. LaMar, E. Moseman, and J. Shook, Threshold Digraphs, arXiv:1212.1149, in review.

[3] C. Greenhill, A Polynomial Bound on the Mixing Time of a Markov Chain for Sampling Regular Directed Graphs, Electronic Journal of Combinatorics 18 (2011), \#P234.

[4] H. Kim, C. I. D. Genio, K. E. Bassler, and Z. Toroczkai, Constructing and sampling directed graphs with given degree sequences, New Journal of Physics 14 (2012), 023012 . 


\section{Topological Models of Power Grids}

\section{Brian Cloteaux}

Over the past two decades, it has been observed that many of the networks embedded in systems all around us have a number of common features. The importance of these networks, commonly called complex networks, lies in the number of areas in which they are found. They have been shown to arise naturally in systems of both biological and social interactions. In addition, there have also been claims that complex networks appear in many engineered systems such as the Internet, software components, and an area in which we are particularly interested, power grids.

Being able to measure and model engineered systems has become an essential step towards being able to maintain and understand the reliability, safety, and in some cases the security of these systems. This is especially true for power grids, where there is a need for formal measurement programs. But this need poses several questions about how we should analyze power grid networks. In order to perform topological analysis on the structure of power grids, these networks are often treated in the literature as being complex networks. But this approach raises the question, "Why would a highly engineered system, such as a power
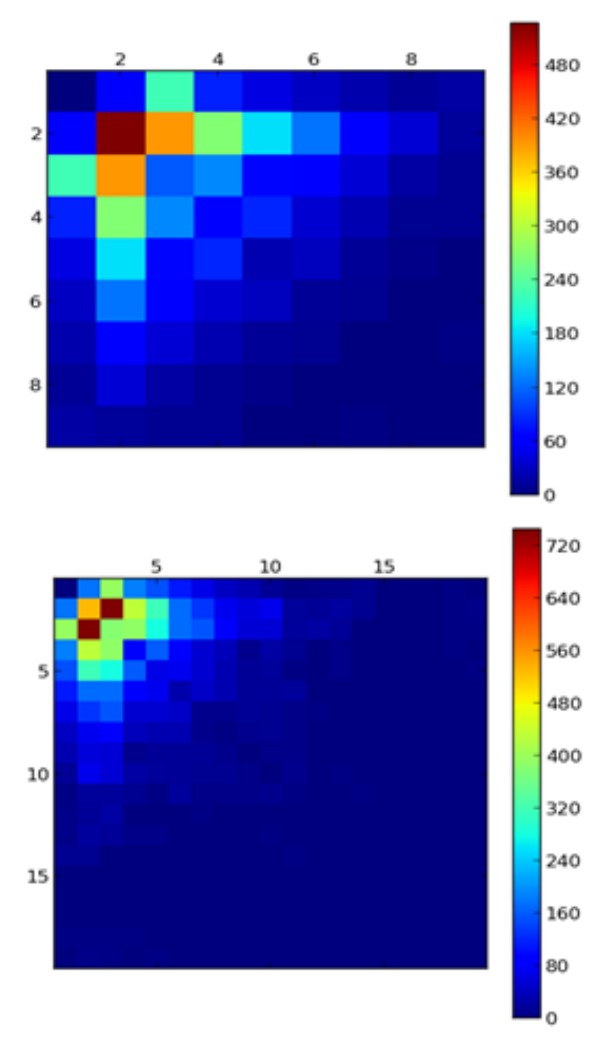

Figure 62. Joint degree distributions. Top: Polish power grid. Bottom: Western US power grid. grid, show characteristics of complex networks?"

In order to understand where the effects of engineering choices show up in power grid networks, we examined three power grids under the $\mathrm{dK}$-hierarchy of network models. The $\mathrm{dK}$-series is a series of progressively refined sets of approximations for networks [3]. By progressively refined, we mean that these models have the property that every member of the $\mathrm{dK}$-set of models for a given network is also a member of the (d-1)K-set of models. As d gets larger, these sets quickly reduce to the target network itself. In modeling a given network, its $1 \mathrm{~K}$-models are defined to be all of the graphs with the network's degree sequence, while its $2 \mathrm{~K}$-models also have the network's joint degree sequence, and finally its $3 \mathrm{~K}$ models also have the network's local clustering coefficients. In order to capture many of the topological characteristics of a network, its random models often must be at least $2 \mathrm{~K}$-models, and sometimes they must even be $3 \mathrm{~K}$-models.

It has been observed that power grids seem to always have an exponential degree distribution with an exponent of 0.5 . This distribution is independent of the underlying engineering principles used in its design. We speculate that this is a result of capacity limitations, combined with consistent population distributions [2]. Thus, we are able to create $1 \mathrm{~K}$-models without consideration of underlying engineering choices.

For $2 \mathrm{~K}$-models, it is a very different story. We have shown a dramatic difference in the joint degree distribution of various power grids. Shown in Figure 62 is the joint degree distribution of the Polish and the Western United States power grids. Comparing these two systems, we see a large difference between the number of two degree node that connect to other two degree nodes. This difference is directly attributable to differing engineering choices made between the two systems. Thus, for us to be able to create a $2 \mathrm{~K}$-model of either of these systems, we must account for the local engineering principles used in the design.

This has led us to develop a new modeling algorithm for creating random power grids similar to the one in the western U.S. By taking into account engineering choices in the design of our random models, we have been able to create more realistic topological models for our power grid research.

[1] B. Cloteaux. Modeling the Topology of Power Grids, in preparation.

[2] B. Jiang and T. Jia, Zipf's Law for all the Natural Cities in the United States: a Geospatial Perspective, International Journal of Geographical Information Science 25:8 (2011), 1269-1281.

[3] P. Mahadevan, D. Krioukov, K. Fall, and A. Vahdat, Systematic Topology Analysis and Generation Using Degree Correlations, SIGCOMM Computer Communications Review 36:4 (2006), 135-146. 


\section{Geometry of Networks}

\author{
Iraj Saniee (Bell Labs) \\ Sean Kennedy (Bell Labs) \\ Onuttom Narayan (Univ. of California at Santa Cruz) \\ Gabriel H. Tucci (Bell Labs) \\ Shi Li (Princeton University) \\ John D. Hobby (Bell Labs) \\ Sean Kennedy (Bell Labs) \\ Jaime Morgenstern (Carnegie Mellon University) \\ Gordon Wilfong (Bell Labs) \\ Lisa Zhang (Bell Labs)
}

Gromov hyperbolicity [4] is important not only in group theory, coarse geometry, and differential geometry, but also in many applied fields such as communication networks, cyber security and statistical physics. Hyperbolicity is observed in many real world networks, such as the Internet and data networks at the IP layer. The study of traffic flow and congestion in graphs is an important subject of research in graph theory. Furthermore, it is an extremely important topic in network theory and more specifically, in the study of communication networks. This research is concerned with investigation of hyperbolicity in large-scale networks, its measurement and detection on real data sets and its impact on flows. We also explore the algorithmic advantages of hyperbolicity to simplify computations on large graphs.

Hyperbolicity of Real-Life Large Networks (Kenne$d y$, Narayan and Saniee). In our earlier work we proposed an experimental device which we called the curvature plot for detection of hyperbolicity in largescale networks. Hyperbolic networks are those in which geodesics (or shortest paths) between any triple set of points are always within a maximum distance $\delta \geq$ 0 of each other ("all triangles are $\delta$-slim"). We had illustrated the utility of curvature plots for large graphs by applying them to graphs of communication networks at the IP layer using publicly available measurements.

In this work, we explore hyperbolicity of more than two dozen networks, ranging over co-authorship, friendship and email graphs, peer-to-peer, Web and Internet graphs, road graphs, and other graphs from a few tens of thousands to several million nodes. We show that all but the road networks exhibit strong hyperbolicity (that is, $\delta$ is considerably smaller than the graph diameter) and in each case we document the statistics of both the hyperbolic constant $\delta$ and the graph diameter. This investigation shows that hyperbolicity is not a specialized property limited to a small class of graphs, but in many ways is universal, at least among large-scale graphs encountered in practice. We conclude with quantification of the algorithmic advantages of hyperbolic graphs for path computation for networks of up to several hundred million nodes. (To be released in 1Q 2013.)

Non-Hyperbolicity in Random Regular Graphs and their Traffic Characteristics (Tucci [1]). In this work we study congestion and hyperbolicity in random regular graphs. By congestion we mean the maximal load on the nodes of a network given a distribution of flows between its nodes. We study traffic congestion under geodesic routing, by which we mean that the path chosen to route the traffic between the nodes is the minimum length path, and tied paths receive equal fraction of each flow. Based on prior work, we would expect that for a hyperbolic network of size $n$, congestion would scale as $\Theta\left(n^{2}\right)$ for uniform traffic. Here we show that for random $d$-regular graphs (i.e., each node has degree $d \geq 3$ ) the maximum congestion scales as $O\left(n \log _{d-1}(n)^{3}\right)$, suggesting these graphs are nonhyperbolic. Further, we show formally that random $d$ regular graphs are not $\delta$-hyperbolic for any nonnegative $\delta$ asymptotically almost surely. We also provide a tighter lower bound on the graph slimness $\delta$ as a function of $n$ (graph size) and $d$ (degree) for $d$-regular graphs than what has been known so far.

Lack of Hyperbolicity in Asymptotic Erdös-Renyi Sparse Random Graphs (Narayan, Saniee and Tucci [2]). Random graphs are the simplest examples of networks with small diameter, in the sense that the largest distance between any pair of points scales like $\log (n)$ for a family of random graphs with $n$ nodes. It is often thought that at least in the sparse regime, that is, when the number of edges is small, these graphs are highly tree-like and are probably hyperbolic. In this work, we prove that the giant component of the Erdös-Renyi random graph is not Gromov $\delta$-hyperbolic for any $\delta$ with probability tending to one as $n$ gets large. As a corollary we provide an alternative proof that the spectral gap of the giant component of these random graphs approaches zero almost surely as $n$ increases.

Traffic Congestion in Expanders, $(\boldsymbol{p}, \boldsymbol{\delta})$-Hyperbolic Spaces and Product of Trees ( $\mathrm{Li}$ and Tucci). In this work we extend the analysis of traffic congestion in Gromov hyperbolic graphs. These are graphs in which geodesics between any triple set of points are always within a maximum distance $\delta>0$ of each other ("triangles are $\delta$-slim"). We study what happens to traffic congestion if we relax the slimness condition so that not all but a fraction of all triangles are $\delta$-slim. More precisely, we say that a metric $(\mathrm{X}, \mathrm{d})$ is $(\mathrm{p}, \delta)$-hyperbolic if for at least a fraction $p$ of the 3-tuples $(\mathrm{u}, \mathrm{v}, \mathrm{w})$ in $\mathrm{X}$ the geodesic triangle $\Delta(\mathrm{uvw})$ is $\delta$-slim. The case $p=1$ corresponds to the classic Gromov $\delta$-hyperbolic spaces. We show that congestion in these graphs scales as $\Omega\left(p^{2} n^{2} / D_{n}^{2}\right)$ where $D_{n}$ is the diameter of $G_{n}$.

An important family of graphs is expanders. In graph theory, an expander is a sparse graph that has 
strong connectivity properties. Expander constructions have spawned research in pure and applied mathematics, with several applications to complexity theory, design of robust computer networks, and the theory of error-correcting codes. It is well known that random regular graphs are a large family of expanders. It was proved in [1] that random d-regular graphs have maximum vertex congestion scaling as $O\left(n \log _{d-1}(n)^{3}\right)$. Therefore, it is a natural question to ask if expanders have always low congestion under geodesic routing. In this work, we show that this is not the case. More precisely, we construct a family of expanders with maximum vertex congestion $\Theta\left(n^{2}\right)$. (To be released in 1Q 2013.)

Traffic Analysis in Random Delaunay Tessellations and Other Graphs (Hobby and Tucci [3]). Given a set of points in Euclidean space, the Delaunay construction gives a canonical triangulation whose vertices are these points. These triangulations have many nice combinatorial and geometric properties that make them extremely useful. Moreover, they can also be constructed in Riemannian manifolds. However, they do not exist for arbitrary sets of points: certain density requirements are needed to ensure that the triangulation can accurately represent both the topology and geometry of the manifold. These triangulations are canonically determined by the set of points and have many of the properties that Delaunay triangulations have in the Euclidean space.

In this paper, we focus on the following construction. Let $\mathrm{M}$ be a either the open unit disk or the whole Euclidean plane and let $\mathcal{P}$ be a Poisson point process with rotationally symmetric density $\rho$ on $\mathrm{M}$. Let $\mathcal{T}$ be the Delaunay triangulation graph of $\mathcal{P}$ with respect to the Euclidean metric. Our interest is in understanding how this graph's characteristics change as we change the density function $\rho$. In particular, we study how the maximum vertex congestion is affected by changes in the function $\rho(x, y)$. We also study how the density affects the degree distribution of the corresponding triangulation as well as other graph properties. Finally, we investigate the maximum vertex congestion in Erdös-Renyi random graphs, random geometric graphs and random d-regular graphs.

Hierarchical Clustering via Räcke Decomposition (Kennedy, Morgenstern, Wilfong and Zhang). Räcke in 2002 identified an effective recursive hierarchical decomposition of a graph, with the property that each cluster of nodes at each level of the hierarchy is internally better connected than to their peers clusters (low cut value) and this property is repeated at each level of the hierarchy. It turns out that the underlying algorithm for Räcke decomposition is NP-hard and subsequent polynomial time algorithms proposed for this decomposition use semi-definite programming and thus are not scalable. In this work, we redefine the cut value with the aim of reducing the complexity of the clustering algorithm. In particular, we replace Räcke's exhaustive search (to find a group of nodes with low cut value) with non-exhaustive approximations and heuristics. We benchmark the resulting algorithm on a test set of networks including synthetic networks with an underlying hierarchical structure. We show our results compare favorably to existing iterative methods for hierarchical decompositions. We expect our approach to lead to more scalable algorithms applicable to large-scale network clustering. (To be released in 1Q 2013.)

[1] G. H. Tucci, Random Regular Graphs are not Asymptotically Gromov Hyperbolic, arXiv: 1203.5069, in review.

[2] O. Narayan, I. Saniee and G. H. Tucci, Lack of Hyperbolicity in Asymptotic Erdös-Renyi Sparse Random Graphs, arXiv: 1009.5700v2, in review.

[3] J. D. Hobby and G. H. Tucci, Traffic Analysis in Random Delaunay Tessellations and Other Graphs, arXiv: 1203.4863, in review.

[4] Jussi Väisälä, Gromov Hyperbolic Spaces, Expositiones Mathematicae 23:3 (2005), 187-231.

This work received partial support from NIST grant 60NANB10D128.

\section{Broadcast and Spread of Information in Complex Networks}

\section{Fern Y. Hunt}

To understand the issues involved in the interaction between the local dynamics of agents in a network and the network topology, we began an investigation of the conditions for optimal spread of information. The research literature on the general topic of spread in networks is encyclopedic and a lot of effort has been devoted to elucidating the relationship between network topology and mode of spread. The understanding gained has broad practical implications, from the design of efficient broadcasting algorithms, to effective strategies against malware, to better understanding of the genesis of cascades.

In 2003 J. Kleinberg et al. posed the problem of optimal spread of opinion or innovation in social networks in terms of finding the set of most "influential" nodes of a given cardinality, i.e. the set of initially activated nodes that generate the largest resulting cascade of activated nodes. Later, in 2010, Borkar et al. studied a related problem where the mode of spread is a simple random walk on the network graph. Despite its simplicity, this model has found wide application, for example in routing algorithms for networks where nodes have limited location information, and more generally in the development of so-called consensus algorithms, where 
spread is due to nearest neighbor interaction. In this setting a target subset of the nodes (representing "consensus leaders") is initially assigned consensus function values that remain fixed. Function values of the remaining nodes are computed iteratively by averaging fixed values from the boundary of the target set and those of its neighbors as dictated by the random walk. Following Borkar we seek to identify the target set of fixed cardinality D for which the time to consensus is shortest. It is convenient to minimize a related time, namely the average expected time it takes for a random walker initially situated outside the target set to reach the set for the first time- that is, the hitting time of the set averaged over all initial positions of the walk. Solving the resulting optimization problem exactly is quite challenging for large graphs and may be NP hard. An approximation to the optimal set constructed by a greedy algorithm was shown by Borkar et al. to be within a factor (1-1/e) of optimal. Our goal is not primarily to devise a better algorithm; rather, we seek to understand the interaction of hitting times and graph topology that are sufficient conditions for the existence of tractable optimal target sets.

Currently our efforts center on finding optimal target sets that are subsets of $k$-dominated subsets of a connected graph. A $k$-dominated subset is a subset of the graph such that every vertex $v$ is either in it or is within distance $k$ of some element of it. Exact calculation of the optimal subsets of a large number of graphs shows that with few exceptions these sets are contained in a $k$-dominated subset. For example the vertex cover of the graph, a special case of $k$-domination for $k=1$, is an optimal target set for $D$, the cardinality of the cover. As a byproduct, we developed a method for finding optimal target sets as subsets of a maximal matching of the graph. To further our goal, we are initially restricting our study to trees and graphs with small treewidth, because a polynomial time algorithm for finding optimal target sets in general graphs may not exist. However, such algorithms do exist for a similar problem for graphs with small treewidth. Moreover, there is a polynomial (in the number of vertices) time algorithm (motivated by the facility location problem in operations research) for computing $k$-dominating subsets or their approximations for this class of graphs and graphs of general topology, so there is the possibility that the results of this research can be applied to a broad range of realistic networks.

[1] F. Y. Hunt, Optimal Spread in Network Models of Consensus, in preparation.

\section{Security Risk Analysis of Enterprise Networks Using Attack Graphs}

\author{
Anoop Singhal (ITL SSD) \\ Xinming Ou (Kansas State University) \\ Rui Zhuang (Kansas State University) \\ Su Zhang (Kansas State University) \\ Pengsu Cheng (Concordia University) \\ Lingyu Wang (Concordia University) \\ Sushil Jajodia (George Mason University) \\ Stephen Noel (George Mason University)
}

http://csrc.nist.gov/groups/SNS/security-risk-analysis-enterprisenetworks/index.html

At present, computer networks constitute the core component of information technology infrastructures in areas such as power grids, financial data systems, and emergency communication systems. Protection of these networks from malicious intrusions is critical to the economy and security of our nation. Vulnerabilities are regularly discovered in software applications, which are exploited to stage cyber attacks. Currently, management of security risk of an enterprise network is more an art than a science. System administrators operate by instinct and experience rather than rely on objective metrics to guide and justify decision making.

The objective of this research is to develop a standard model for measuring security of computer networks. A standard model will enable us to answer questions such as "Are we more secure now than yesterday?", or "How does the security of one network configuration compare with another one?" Also, having a standard model to measure network security will allow users, vendors and researchers to evaluate methodologies and products for network security in a coherent and consistent manner.

We have approached the challenge of network security analysis by capturing vulnerability interdependencies and measuring security in the exact way that real attackers penetrate the network. Our methodology for security risk analysis is based on the model of attack graphs. We analyze all attack paths through a network, providing a probabilistic metric of the overall system risk. Through this metric, we analyze trade-offs between security costs and security benefits.

In FY2012, we integrated our techniques into an attack graph-based security tool called MULVAL, which we released as an open source system. We also published a SpringerBrief book [1] on this topic.

Aggregating Common Vulnerability Scoring System $^{26}$ (CVSS) scores of individual vulnerabilities provides a practical approach to network security metrics. However, existing approaches to aggregating

${ }^{26}$ http://www.first.org/cvss 
CVSS scores usually result in useful semantics of individual scores to be lost in the aggregated result. We published a new paper [2] in which we address this issue in two novel ways. First, instead of taking each base score as an input, our approach drills down to the underlying base metric level where dependency relationships have well defined semantics. Second, our approach interprets and aggregates base metrics in a way to preserve the corresponding semantics of individual scores.

Moving-target defense has been hypothesized as a potential game changer in cyber defense, including that for computer networks. However there has been little work to study how much proactively changing a network's configuration can increase the difficulty for attackers and thus improve the resilience of the system under attack. This year we presented a basic design schema for a moving-target network defense system [3]. Based on this design schema, we conducted a simulation study to investigate the degree to which proactively changing a network's parameters can decrease an adversary's chance for success. We believe this is an important first step towards understanding why and how the concept of a moving target can be successfully applied to computer network defenses.

Finally, we also developed a new metric, "K Zero Day Safety," for measuring security risk of a network against unknown vulnerabilities [4].

In FY2013, we plan to apply attack graphs to study the effectiveness of moving target defense. We also plan to publish our results as a NIST report and as papers in conferences and journals.

[1] X. Ou and A. Singhal, Quantitative Security Risk Assessment of Enterprise Networks, SpringerBriefs in Computer Science, Springer, New York, December 2011.

[2] P. Cheng, L. Wang, S. Jajodia, and A. Singhal, Aggregating CVSS Base Scores for Semantics-Rich Network Security Metrics, in Proceedings of the $31^{\text {st }}$ IEEE International Conference on Reliable and Distributed Systems, Irvine, CA, October 2012.

[3] R. Zhuag, S. Zhang, S. Deloach, X. Ou, and A. Singhal, Simulation-based Approaches to Studying Effectiveness of Moving Target Network Defense, in Proceedings of the National Symposium on Moving Target Defense, Annapolis, MD, June 2012.

[4] L. Wang, A. Singhal, S. Jajodia, and S. Noel, A Network Security Metric for Measuring and Improving Computer Networks Resistance to Unknown Vulnerabilities, in review.

This research was performed in part with funding from ACMD.

\section{Distributed Motion Control in Mobile Sensor Networks}

\author{
V. Marbukh \\ K. Sayrafian \\ A.G. Aghdam (Concordia University) \\ H. Mahboubi (Concordia University)
}

A wireless sensor network is a set of spatially distributed sensors that monitor some physical targets (e.g. people) or environmental conditions, such as temperature, sound, pressure, etc. and cooperatively pass their data to a desired location through wireless connections in the network. A mobile sensor network (MSN) is a wireless sensor network in which each node is autonomously capable of moving or relocating to a new position. This mobility adds a new dimension to the capabilities of sensor networks and allows them to better adapt to changes in the environment where they are operating.

Locations of sensors in a MSN affect both their ability to better acquire information on the intended target(s) and event(s) as well as their ability to communicate this information to the intended recipient(s). The information acquisition needs, which require proximity to the target(s), often compete with the communication needs, which require proximity to the recipient(s) of the sensor information. Lack of centralized control, variety of performance criteria and operational uncertainties, and possibilities of MSN topology change in a dynamic environment make MSN optimization an exceedingly challenging problem. In addition, there are a number of practical constraints, such as energy consumption and obstacle avoidance, which have to be taken into account in this regard.

In our research, we have proposed aligning sensor mobility with the overall MSN performance goals by assuming that (a) sensing and communication are optimized much faster than sensors relocate, and (b) sensor relocations are governed by cost/benefit analysis, where "cost" of sensor battery energy expenditure for sensor relocation is weighted against projected performance gains due to new sensor locations. Based on Voronoi-partitioning, we have developed sensor deployment strategies for effective coverage in wireless sensor networks. The relocation strategy is based on monitoring the distances of the sensors and the points inside its corresponding Voronoi polygon from the edges or vertices of the polygon [1]. Extensive simulations have confirmed the effectiveness of our proposed deployment algorithms, and their superiority to alternate techniques reported in the literature.

We have also investigated energy-efficient techniques for tracking a target in a field while maximizing the life-span of the network. The major sources of energy depletion in the network are assumed to be sensing, communication and movement of the sensors. 
The problem is formulated as a constrained optimization, and a procedure for solving this optimization problem has been outlined in [2].

For the future, we intend to investigate the problem of tracking a target in a field with obstacles using cooperative mobile sensors in an energy-efficient manner. It is desired to develop a strategy to relocate the sensors to optimal positions at any time instant such that the total energy consumption of the sensors is minimal. This will ensure sufficient network lifetime, which is an essential design consideration for MSNs.

[1] H. Mahboubi, A. Momeni, A.G. Aghdam, K. SayrafianPour, and V. Marbukh, An Efficient Target Monitoring Scheme with Controlled Node Mobility for Sensor Networks, IEEE Transactions on Control Systems Technology 20 (6) (2012)1522-1533.

[2] H. Mahboubi, W. Masoudimansour, A. G. Aghda, K. Sayrafian, and V. Marbukh, Maximum Life Span Strategy for Target Tracking in Mobile Sensor Networks, in Proceedings of 2012 American Control Conference, Montreal, Canada, June 2012, 5096-5101.

\section{Combinatorial Testing}

\section{Raghu Kacker \\ Rick Kuhn \\ James Lawrence \\ Yu Lei (University of Texas at Arlington) \\ Itzel Mendoza (Centro Nacional de Metrologia)}

Combinatorial testing (CT) is an approach to detect combinatorial interaction faults, which may cause a software-based system to fail when some particular values of certain parameters combine with some particular values of certain other parameters. Many critical system failures in recent times have been traced to interaction faults in the underlying software. CT is a versatile and broadly applicable methodology for ensuring trustworthy software in business, industrial, medical, scientific, and transport systems.

It is often difficult or impossible to characterize all possible combinatorial interactions, let alone test them. Combinatorial testing is based on the insight that while the behavior of a software system may be affected by a large number of factors, only a few factors are involved in a failure-inducing fault. Investigations of actual faults show that most faults involved a single parameter, a smaller proportion of faults resulted from interactions between values of two parameters, and progressively fewer interaction faults involved $3,4,5$, and 6 parameters. Most combinatorial testing has until recently been limited to pair-wise (2-way) testing, in which all interactions involving two parameters are detected. A reason is that efficient test methods and tools to detect higher order interaction faults have so far not been available. The goal of this project is to advance the technology from pair-wise to higher order (strength) testing and to demonstrate successful applications of high strength combinatorial testing.

We have recently completed a major revision to the NIST/UTA software tool Advanced Combinatorial Testing System (ACTS) for generating high strength combinatorial test suites with support of constraints. We have distributed 1,250 copies worldwide to companies and individuals. We estimate the number of users of ACTS tool to be several thousand. We continue to provide consulting services upon request.

Comparison benchmarking. We compared ACTS to other free research tools. We used both reallife systems collected from the literature, as well as some synthesized systems. The comparison shows that ACTS performs better in most cases, especially for larger systems with more complex constraints.

Third-party constraints solver. We integrated the open-source constraints solver Choco in ACTS for better and wider constraints support. This is written in Java and is platform-independent. Previously (201011 ), we had integrated constraints solver $Z 3$ developed by Microsoft which is specific for Windows, which made widespread use of ACTS more difficult.

Introduction to Combinatorial Testing. We have converted our NIST Special Publication on "Practical Combinatorial Testing" into a draft for a book to be published by Taylor and Francis Publishers, with additional invited chapters, in 2013.

Mini-symposium on combinatorial testing. We organized the First International Workshop on Combinatorial Testing (IWCT2012) in conjunction with the 5th IEEE International Conference on Software Testing, Verification, and Validation (ICST2012) held in April 2012 in Montreal. Organizers were Itai Segall, Rick Kuhn, Raghu Kacker, and Yu Lei. We solicited papers from industry and academia and received 20 submissions; 11 were selected by the Program Committee. We had a good balance of papers from industry and academia. The IWCT2012 had over 30 participants, and we had a good exchange of technical information. The organizers of ICST2013 have invited us to do another workshop in conjunction with ICST2013.

Modeling of the input test space. We have done a case study on the use of CT by using the ACTS tool itself as the software system under test. The study illustrates the challenges in modeling the input space [1].

Identification of failure-inducing combinations. We have developed a novel iterative approach to identify failure-inducing combinations. A tool called "Ben" to help automate fault localization is under development. We have also investigated use of a classification tree approach to identify failure-inducing combinations. A research paper on this approach was presented at IWCT2012 [2]. 
Combinatorial coverage measurement. We have developed a prototype tool to determine combinatorial coverage of candidate test suites. The earlier version evaluated up to 4-way combinations. We have improved the tool to evaluate 5-way and 6-way combinations. A guest researcher from Mexico, Itzel Mendoza, is working with us to incorporate support of constraints in the NIST Combinatorial Coverage measurement tool.

Combinatorial testing for electronic healthcare systems. We are investigating the benefits of combinatorial testing for conformance and interoperability testing of healthcare systems. In particular we are applying CT to an implementation of the ISO/IEEE 11073 communication protocol for health informatics. Antidote is an open-source implementation of ISO/IEEE 11073. We have built a tool to input test sequence messages to Antidote. We plan to generate a set of combinatorial sequences. We will use the testing tool to apply these sequences, and then measure the effectiveness of these sequences in terms of code coverage and/or fault detection.

[1] M. Borazjany, L. Yu, Y. Lei, R. Kacker and D. R. Kuhn, Combinatorial Testing of ACTS: A Case Study, in Proceedings of the Fifth IEEE International Conference on Software Testing, Verification and Validation, First International Workshop on Combinatorial Testing (IWCT) Montreal Canada April 17-21, 2012, 591-600.

[2] K. Shakya, T. Xie, N. Li, Y. Lie, R. Kacker and D. R. Kuhn, Isolating Failure-Inducing Combinations in Combinatorial Testing uses Test Augmentation and Classification, in Proceedings of the Fifth IEEE International Conference on Software Testing, Verification and Validation, First International Workshop on Combinatorial Testing (IWCT) Montreal Canada, April 17-21, 2012, 620-623.

\section{Approximating the Ising Partition Function}

\section{Isabel Beichl \\ Amanda Streib \\ Noah Streib \\ Francis Sullivan (IDA Center for Computing Sciences)}

The Ising model is a classical model in the statistical mechanics of magnetization. Historically, the Ising model has played a significant role in physics; the discovery of phase transitions within the model served as one of the first pieces of evidence in support of atomism, the claim that small changes on an atomic scale could lead to large, observable changes. Due to its subtle complexities, the Ising model continues to be studied intensely by mathematicians, computer scientists, and physicists alike.

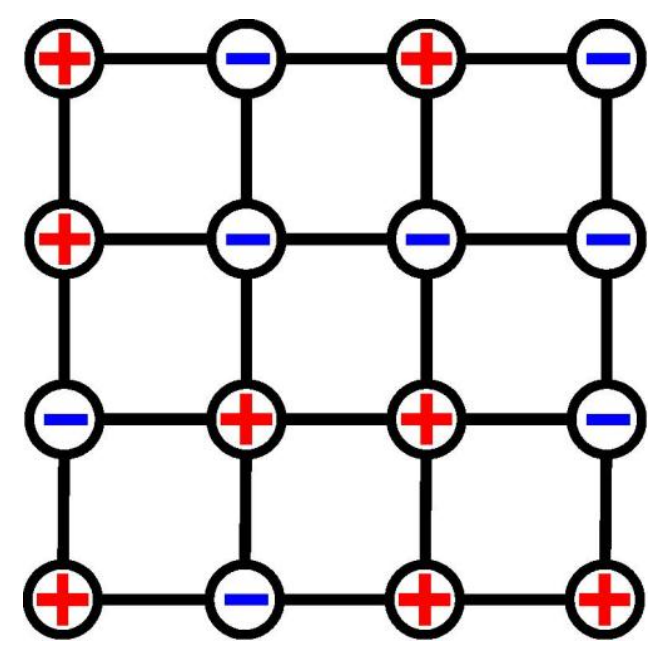

Figure 63. Lattice with spin configuration $\sigma$.

The Ising model was one of the origins of the socalled Monte Carlo method and one of the first examples of a computational problem that is provably hard. Computational ideas and techniques that originated in studies of the Ising model continue to be relevant to current work in biosciences, material science, the understanding of networks, nanotechnology, and behavior of colloids.

The Ising model represents a magnet acted on by an external field. The magnet is represented by a graph $G=(V, E)$, where the vertices $V$ are the atoms in the magnet. The graph is accompanied by an assignment $\sigma$ of $\{ \pm 1\}$ spins to each vertex, called spin configurations, which represent the possible states of the atoms in the magnet. Each spin configuration appears in the model with a certain probability, which is governed by the temperature, the strength and direction of the external field, and the correlation of the spins between neighboring atoms. Given values for these parameters, the (Boltzmann) probability distribution on the $2^{|V|}$ spin configurations is defined as: $\pi(\sigma)=f(\sigma) / Z$. The denominator $Z$, which is the sum of the $f(\sigma)$ over all spin configurations, is called the partition function. Since an estimate of the partition function yields estimates for many important physical and thermodynamic quantities of the magnet, it is the partition function that we strive to find.

Unfortunately, finding $Z$ is hard. Except for a few very-restricted special cases, the problem of determining $Z$ exactly is \#P complete. Therefore, we seek to find approximation algorithms for $Z$ that run quickly. Currently, physicists implement a Markov chain called the Metropolis algorithm [4], despite the fact that it has been proven to be inefficient at low temperatures! A provably polynomial algorithm is a Markov chain of Jerrum and Sinclair [2]. However, this algorithm is again too slow to use in practice. 
Our aim has been to design an algorithm that physicists can use in practice. The algorithm that we created does just that. It combines a heuristic sampling method of Chen [1] (which generalizes the pioneering work of Knuth [3]) with graph-theoretic and linear-algebraic tools. One of the serious issues with existing work on this problem is that convergence becomes slower and slower near a critical temperature. We have developed a radically new method that is temperature independent. The algorithm generates coefficients that, once computed, can be used for any temperature.

[1] P. Chen, Heuristic Sampling: a Method for Predicting the Performance of Tree Searching Programs, SIAM Journal on Computing 21 (1992), 295-315.

[2] M. Jerrum and A. Sinclair, Polynomial-time Approximation Algorithms for the Ising Model, SIAM Journal on Computing 22:5 (1993), 1087-1116.

[3] D. E. Knuth, Estimating the Efficiency of Backtrack Programs, in Selected Papers on Analysis of Algorithms, CSLI Publications, Stanford, CA (2000).

[4] N. Metropolis, A. W. Rosenbluth, M. N. Rosenbluth, A. H. Teller, and E. Teller, Equations of State Calculations by Fast Computing Machines, Journal of Chemical Physics 21:6 (1953), 1087-1092.

\section{Spectral Algorithms for Document Topic Modeling}

\author{
Yi-Kai Liu \\ Anima Anandkumar (University of California, Irvine) \\ Dean Foster (University of Pennsylvania) \\ Daniel Hsu (Microsoft Research - New England) \\ Sham Kakade (Microsoft Research - New England)
}

An important task in machine learning is to take a collection of documents and classify them according to their underlying topics. This poses a number of difficulties. In particular, one may not know a priori what topics will appear in the documents, and moreover, each individual document can contain a mixture of several topics. Latent Dirichlet allocation (LDA) is a well-known model for this situation. Typically one uses expectation maximization (EM) to learn the parameters of the LDA model, and then uses this model to classify the documents. Unfortunately, the EM algorithm can be computationally expensive, and may not converge to a globally optimal solution in general.

We have developed a spectral algorithm for learning the LDA model, which works by estimating the third-order moments of the word distributions of the documents, and computing an orthogonal tensor decomposition (a generalization of the singular value decomposition). This algorithm is guaranteed to recover the parameters of the LDA model exactly, and it can be implemented efficiently using linear algebra techniques. Numerical experiments suggest that our spectral algorithm is competitive with existing optimized implementations of the EM method. Interestingly, there are similarities between the tensor decomposition used in our algorithm, and certain classes of many-body quantum states.

[1] A. Anandkumar, D. P. Foster, D. Hsu, S. M. Kakade and Y.-K. Liu, A spectral algorithm for latent Dirichlet allocation, in Advances in Neural Information Processing Systems, Volume 25 (P. Bartlett and F.C.N. Pereira and C.J.C. Burges and L. Bottou and K.Q. Weinberger, eds.), 2012. 


\section{Part IV}

\section{Activity Data}

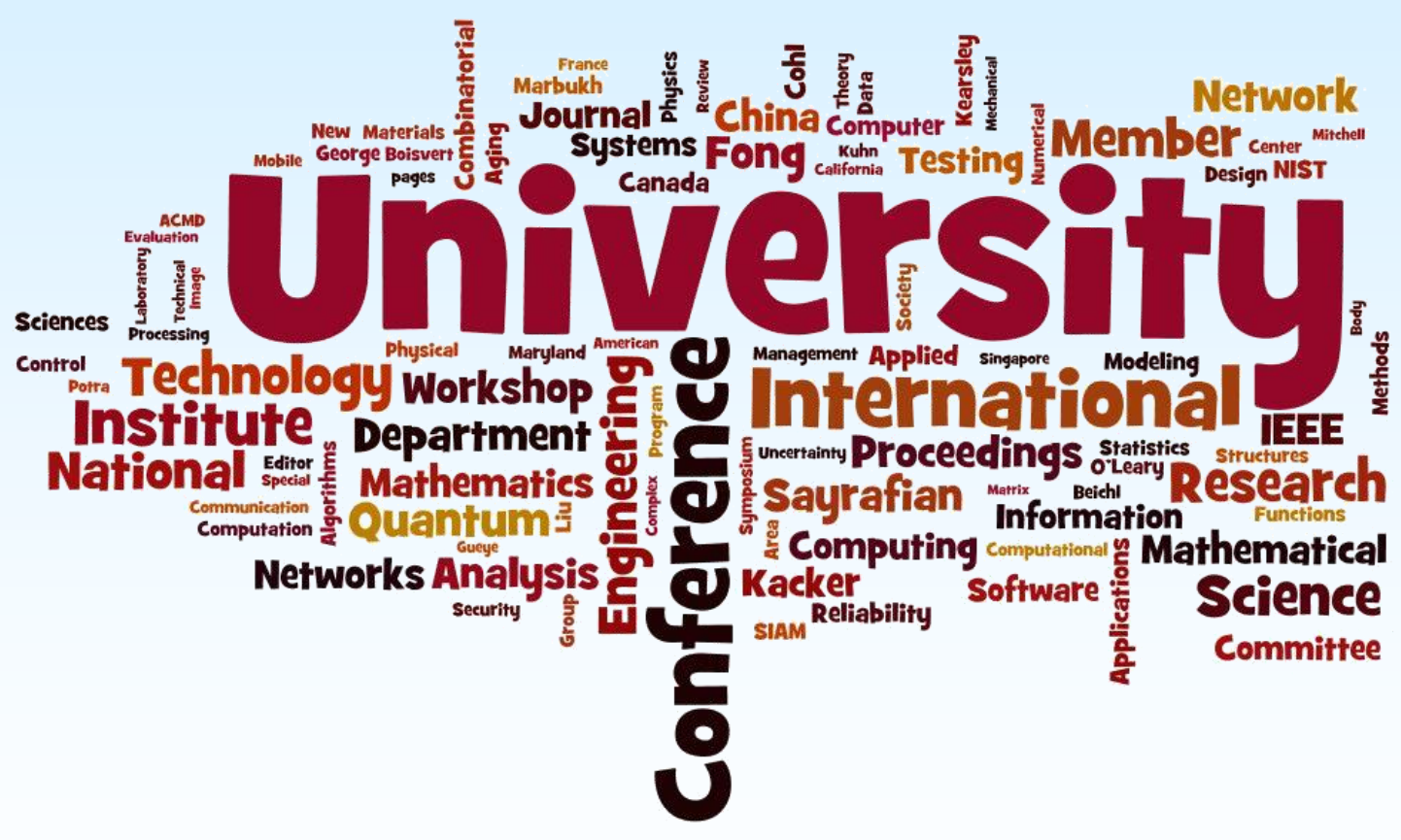




\section{Publications}

Note: Names of (co-)authors with a current Division affiliation are underlined.

\section{Appeared}

\section{Refereed Journals}

1. G. S. Abo, Y. K. Hong, B. C. Choi, M. J. Donahue, S. Bae, J. Jalli, J. Park, J. Lee, M.-H. Park and S.-H. Gee, Micromagnetic Computer Simulated Scaling Effect of S-shaped Permalloy Nanoelement on Operating Fields for AND or OR logic, IEEE Transactions on Magnetics 48 (2012), 18511855 .

2. B. K. Alpert, W. Bertrand Doriese, Joseph W. Fowler and Joel N. Ullom, Predicted Energy Resolution of a Running-Sum Algorithm for Microcalorimeters, Journal of Low Temperature Physics 167:5-6 (2012) 582-587.

3. I. Beichl, E. Moseman and F. Sullivan, Computing Network Reliability Coefficients, Congressus $\mathrm{Nu}$ merantium 207 (2011), 111-127.

4. D. A. Bennett, R. D. Horansky, D. R. Schmidt, A. S. Hoover, R. Winkler, B. K. Alpert, J. A. Beall, W. B. Doriese, J. W. Fowler, C. P. Fitzgerald, G. C. Hilton, K. D. Irwin, V. Kotsubo, J. A. B. Mates, G. C. O’Neil, M. W. Rabin, C. D. Reintsema, F. J. Schima, D. S. Swetz, L. R. Vale, J. N. Ullom, A High Resolution Gamma-ray Spectrometer Based on Superconducting Microcalorimeters, Review of Scientific Instruments 83 (2012), 093113.

5. R. J. Braun, R. Usha, G. B. McFadden, T. A. Driscoll, L. P. Cook and P. E. King-Smith, Thin Film Dynamics on a Prolate Spheroid with Application to the Cornea, Journal of Engineering Mathematics 73 (2012), 121-138.

6. G. W. Bryant, Natalia Malkova, J. Sims, M. Zielinski, W. Jaskolski and J. Aizpurua, Controlling the Optics of Quantum Dots with Nanomechanical Strain, Physical Review B:Condensed Matter and Materials Physics 84 (2011), 235412.

7. J. D. Benson, A. J. Kearsley and A. Z. Higgins, Mathematical Optimization of Procedures for Cryoprotectant Equilibration using a Toxicity Cost Function, Cryobiology 64:3 (2012), 144-151.

8. T. J. Burns, S. P. Mates, R. L. Rhorer, E. P. Whitenton and D. Basak, Modelling the Peak Cutting Temperature during High-speed Machining of AISI 1045 Steel, Engineering Transactions 60 (2012), 118-124.
9. A. S. Carasso, Reconstructing the Past from Imprecise Knowledge of the Present: Effective Nonuniqueness in Solving Parabolic Problems Backward in Time, Mathematical Methods in the Applied Sciences (2012), Wiley OnLine Library, DOI:10.1002/mma2582.

10. B. Cloteaux and L. A. Valentin, Counting the Leaves of Trees, Congressus Numerantium 207 (2011), 129-139.

11. V. R. Coffman, A. C. E. Reid, S. A. Langer, G. Dogan, OOF3D: An Image-based Finite Element Solver for Materials Science, Mathematics and Computers in Simulation 82 (2012), 2951.

12. H. S. Cohl, Fundamental Solution of Laplace's Equation in Hyperspherical Geometry, Symmetry, Integrability and Geometry: Methods and Applications 7 (2011), 108.

13. H. S. Cohl and E. G. Kalnins, Fourier and Gegenbauer Expansions for a Fundamental Solution of the Laplacian in the Hyperboloid Model of Hyperbolic Geometry, Journal of Physics A: Mathematical and Theoretical 45:14 (2012), 145206.

14. H. S. Cohl, Table Errata: "Formulas and Theorems for the Special Functions of Mathematical Physics" by W. Magnus, F. Oberhettinger and R. P. Soni, Mathematics of Computation 81:280 (2012), 2251.

15. H. S. Cohl and H. Volkmer, Eigenfunction Expansions for a Fundamental Solution of Laplace's Equation on $\mathrm{R}^{3}$ in Parabolic and Elliptic Cylinder Coordinates, Journal of Physics A: Mathematical and Theoretical 45:35 (2012), 355204 (20 pages).

16. H. S. Cohl, Erratum: "Developments in Determining the Gravitational Potential using Toroidal Functions, Astronomische Nachrichten 333:8 (2012), 784-785.

17. H. S. Cohl and H. Volkmer, Definite Integrals using Orthogonality and Integral Transforms, Symmetry, Integrability and Geometry: Methods and Applications 8 (2012), 077.

18. G. Dogan and R. H. Nochetto, First Variation of the General Curvature Dependent Surface Energy, ESAIM: ESAIM: Mathematical Modelling and Numerical Analysis 46:1 (2012), 59-79.

19. M. J. Donahue, Micromagnetic Investigation of Periodic Cross-tie/vortex Wall Geometry, Advances in Condensed Matter Physics (2012), 908692 (8 pages).

20. W. B. Doriese, B. K. Alpert, J. W. Fowler, G. C. Hilton, A. S. Hojem, K. D. Irwin, C. D. Reintse- 
ma, D. R. Schmidt, G. M. Stiehl, D. S. Swetz, J. N. Ullom and L. R. Vale, Optimization of the TESbias Circuit for a Multiplexed Microcalorimeter Array, Journal of Low Temperature Physics 167:5-6 (2012) 595-601.

21. S. T. Flammia, D. Gross, Y.-K. Liu and J. Eisert, Quantum Tomography via Compressed Sensing: Error Bounds, Sample Complexity and Efficient Estimators, New Journal of Physics 14 (2012), 095022.

22. J. P. Gaebler, A. M. Meier T. R. Tan, R. Bowler, Y. Lin, D. Hanneke, J. D. Jost, J. P. Home, E. Knill, D. Leibfried and D. J. Wineland, Randomized Benchmarking of Multiqubit Gates, Physical Review Letters 108 (2012), 260503 (5 pages).

23. T. Gerrits, M. J. Stevens, B. Baek, B. Calkins, A. Lita, S. Glancy, E. Knill, S. W. Nam, R. P. Mirin, R. H. Hadfield, R. S. Bennink, W. P. Grice, S. Dorenbos, T. Zijlstra, T. Klapwijk and V. Zwiller, Generation of Degenerate, Factorizable, Pulsed Squeezed Light at Telecom Wavelengths, Optics Express 19 (2011), 24434-24447.

24. S. Glancy, E. Knill and M. Girard, Gradient-Based Stopping Rules for Maximum-Likelihood Quantum-State Tomography, New Journal of Physics 14 (2012), 095017 (9 pages).

25. F. Gurtuna, C. Petra, F. A. Potra, O. Shevchenko and A. Vancea, Corrector-predictor Methods for Sufficient Linear Complementarity Problems, Computational Optimization and Applications 48 (3) (2011), 453-485.

26. C. Hagwood, J. Bernal, M. Halter, J. Elliott, Evaluation of Segmentation Algorithms on Cell Populations using CDF Curves, IEEE Transactions on Medical Imaging 31 (2012), 380-390.

27. P. D. Hale J. Jargon, C. M. Wang, B. Grossman, M. Claudius, J. Torres, A. Dienstfrey and D. Williams, A Statistical Study of De-embedding Applied to Eye Diagram Analysis, IEEE Transactions on Instrumentation and Measurement 61:2 (2012), 475-488.

28. T. M. Hanna, E. Tiesinga, W. F. Mitchell and P. S. Julienne, Resonant Control of Polar Molecules in an Optical Lattice, Physical Review A 85:2 (2012), 022703.

29. C. Holloway, E. Kuester and A. Dienstfrey, Characterizing Metafilms: The Connection Between Surface Susceptibilities and Effective Material Properties, IEEE Antennas and Wireless Propagation Letters 10 (2012), 1507-1511.
30. S. P. Jordan, K. S. M. Lee and J. Preskill, Quantum Algorithms for Quantum Field Theories, Science 336:6085 (2012), 1130-1133.

31. S. P. Jordan, H. Kobayashi, D. Nagaj and H. Nishimura, Perfect Completeness in Classicalwitness Quantum Merlin-Arthur Proof Systems, Quantum Information and Computation 12:5/6 (2012), 460-470.

32. R. Kessel, R. Kacker and K. D. Sommer, Uncertainty Budgeting for Range Calibration, Measurement 45 (2012), 1661-1669.

33. Z. Levine, A. Pintar, J. Hagedorn and C. Fenimore, Uncertainties in RECIST as a Measure of Volume for Lung Nodules and Liver Tumors, Medical Physics 39:5 (2012), 2628-37.

34. Z. H. Levine, B. R. Galloway, A. P. Peskin, C. P. Heussel and J. J. Chen, Tumor Volume Measurement Errors of RECIST Studied with Ellipsoids, Medical Physics 38:5 (2011), 2552-2557.

35. H. Mahboubi, A. Momeni, A.G. Aghdam, K. Say$\underline{\text { rafian }}$ and $\underline{\text { V. Marbukh, An Efficient Target }}$ Monitoring Scheme with Controlled Node Mobility for Sensor Networks, IEEE Transactions on Control Systems Technology 20:6 (2012), 15221533.

36. L. Ma, O. Slattery, Xiao Tang, Single Photon Frequency Up-conversion and its Applications, Physics Reports 521:2 (2012), 69-94.

37. N. Martys, M. Khalil, W. George, D. Looten, P. Hebraud, Stress Propagation in Colloidal Suspensions Under Shear, European Physical Journal E 35:3 (2012), 1-7.

38. L. A. Melara and A. J. Kearsley The radius of attraction for Newton's method and TVminimization image denoising, Applied Mathematics Letters 25:12 (2012), 2417-2422.

39. A. Mink and A. Nakassis, LDPC for QKD Reconciliation, The Computing Science and Technology International Journal 2:2 (2012).

40. M. Mullan and E. Knill, Improving Quantum Clocks via Semidefinite Programming, Quantum Information and Computation 12 (2012), 553-574.

41. J. S. Pelc, L. Ma, C. R. Phillips, Q. Zhang, C. Langrock, O. Slattery, X. Tang and M. M. Fejer, Long-wavelength-Pumped Upconversion Singlephoton Detector at $1550 \mathrm{~nm}$ : Performance and Noise Analysis, Optics Express 19:22 (2011), 21445.

42. J. S. Pelc, Paulina S. Kuo, O. Slattery, L. Ma, Xiao Tang and M. M. Fejer, Dual-channel, Singlephoton Upconversion Detector at $1.3 \mu \mathrm{m}$, Optics 
Express 20:17 (2012), 19075.

43. F. A. Potra, Weighted Complementarity Problems - a New Paradigm for Computing Equilibria, SIAM Journal on Optimization 22:4 (2012), 1634-1654.

44. F. A. Potra and H. Engler, A Characterization of the Behavior of the Anderson Acceleration on Linear Problems, Linear Algebra and its Applications 438:3 (2013), 1002-1011.

45. K. Sayrafian, J. Hagedorn, W. Yang and J. Terrill, A Virtual Reality Platform to Study RF Propagation in Body Area Networks, CogInfoCom 2012, the 3rd IEEE International Conference on Cognitive Infocommunications.

46. K. Sayrafian and K. Y. Yazdandoost, Wireless Technologies in Healthcare: Selected Papers from IEEE PIMRC 2011, International Journal on Wireless Information Networks 19:3 (2012), 159162.

47. W. Villanueva, W. J. Boettinger, G. B. McFadden and J. A. Warren, A Diffuse-Interface Model of Reactive Wetting with Intermetallic Formation, Acta Materialia 60 (2012), 3799-3814.

48. D. F. Williams, M. T. Ghasr, B. Alpert, Z. Shen, A. Arsenovic, R. M. Weikle II and R. Zoughi, Legendre Fit of the Reflection Coefficient of a Radiating Rectangular Waveguide Aperture, IEEE Transactions on Antennas and Propagation 60:8 (2012) 4009-4014.

49. W. Yang and K. Sayrafian, Interference Mitigation Using Adaptive Schemes in Body Area Networks, International Journal on Wireless Information Networks 19:3 (2012), 193-200.

50. Y. Zhang, S. Glancy and E. Knill, Asymptotically Optimal Data Analysis for Rejecting Local Realism, Physical Review A 84 (2011), 062118 (10 pages).

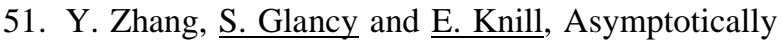
optimal data analysis for rejecting local realism, Physical Review A 84 (2012), 062118 (10 pages).

\section{Journal of Research of NIST}

1. A. S. Carasso and A. E. Vladar, Fractional Diffusion, Low Exponent Levy Stable Laws and 'Slow Motion' Denoising of Helium Ion Microscope Imagery, Journal of Research of the NIST 117 (2012), 199-142.

2. R. Kessel, R. Kacker and K. D. Sommer, Combining Results from Multiple Evaluations of the Same Measurand, Journal of Research of the NIST 116 (2011), 809-820.

\section{Books}

1. A. M. Dienstfrey and R. F. Boisvert, eds., Uncertainty Quantification in Scientific Computing, IFIP) Advances in Information and Communication Technology 377, Springer, Heidelberg, 2012.

\section{Sponsored Research}

2. X. Ou and A. Singhal, Quantitative Security Risk Assessment of Enterprise Networks, SpringerBriefs in Computer Science, Springer, New York, December 2011.

\section{Book Chapters}

1. T. Gründling, W. E. Wallace, C. Barner-Kowollik, C. M. Guttman, A. J. Kearsley, Automated Data Processing and Quantification in Polymer Mass Spectrometry, in Mass Spectrometry in Polymer Chemistry (C. Barner-Kowollik, T. Gründling, J. Falkenhagen, S. Weidner, eds.), Wiley-VCH Verlag \& Co, Weinheim, Germany, 2012.

2. R. Kacker, R. Kessel and K. D. Sommer, Only Non-Informative Bayesian Prior Distributions Agree with the GUM Type A Evaluations of Input Quantities, in Advanced Mathematical and Computational Tools in Metrology and Testing 9 (F. Pavese, M. Bär, J.-R. Filtz, A. B. Forbes, L. Pendrill and H. Shirono, eds.), Series on Advances in Mathematics for Applied Sciences 84, World Scientific, Singapore, 2012, 216-223.

3. D. R. Kuhn, R. Kacker and Yu Lei, Combinatorial Testing, in Encyclopedia of Software Engineering (Phillip Laplante ed.), Online Edition, Taylor and Francis, New York, June 2012, 12 pages.

\section{Conference Proceedings}

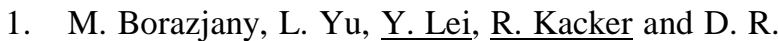
Kuhn, Combinatorial Testing of ACTS: A Case Study, in Proceedings of the Fifth IEEE International Conference on Software Testing, Verification and Validation, First International Workshop on Combinatorial Testing (IWCT) Montreal Canada April 17-21, 2012, 591-600.

2. T. J. Burns, S. P. Mates, R. L. Rhorer, E. P. Whitenton and D. Basak, Transient Convectiondiffusion Modelling of Peak Temperature in Orthogonal Cutting, in Proceedings of the 23rd International Congress of Theoretical and Applied Mechanics (ICTAM2012), Beijing, China, August 19-24, 2012.

3. C. Crosti, D. Duthinh, J. Harris, L.T. Phan, F. A. Potra and E. Simiu, Wind engineering in a multihazard context: probabilistic, synergy and optimi- 
zation issues, in Proceedings of the 13th International Conference on Wind Engineering, IAWE, Amsterdam, The Netherlands, 2011 (8 pages).

4. A. Dienstfrey, Computation, Measurement, and Standards, in Proceedings of the NAFEMS North America Conference, Herndon, VA, September 10-12, 2012.

5. J. T. Fong, Composites Failure Criteria, Uncertainty Propagation and Estimation of the A-basis and B-basis Design Allowables, in Proceedings of the 2012 JEC/Europe Composites Forum, Paris, France, March 26-30, 2012, JEC Composites Magazine 71 (March 2012), 87-90.

6. J. T. Fong, N. A. Heckert and J. J. Filliben, A Stochastic and Local Theory of Fatigue for Aging Pressure Vessels and Piping, in Proceedings of the $3^{\text {rd }}$ International Symposium on Structural Integrity (ISSI), Jinan, China, October 31 - Nov. 2, 2012, pp. 19-31.

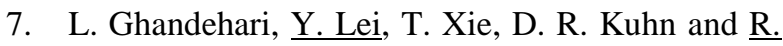
Kacker, Identifying Failure-Inducing Combinations in a Combinatorial Test Set, in Proceedings of the Fifth IEEE International Conference on Software Testing, Verification and Validation (ICST), Montreal Canada, April 17-21, 2012, 370379.

8. A. Gueye and V. Marbukh. A Game Theoretic Framework for Network Vulnerability Assessment and Mitigation, in $3^{\text {rd }}$ Conference on Conference on Decision and Game Theory for Security, Budapest, Hungary November 5-6, 2012.

9. A. Gueye, V. Marbukh and Jean C. Walrand. Towards a Metric for Communication Network Vulnerability to Attacks: A Game Theoretic Approach, in $3^{\text {rd }}$ International ICST Conference on Game Theory for Networks (GameNets), Vancouver, Canada, May 25-26, 2012.

10. D. Harris, F. Sullivan, and I. Beichl, Linear Algebra and Sequential Importance Sampling for Network Reliability, in Proceedings of the Winter Simulation Conference, Phoenix AZ, December 11-14, 2011, 3344-3352.

11. R. Kacker, D. R. Kuhn, Y. Lei and J. Lawrence, Combinatorial Testing for Software Used in Metrology, in Proceedings of the 20-th IMEKO (International Measurement Confederation) World Congress, Busan, Korea, September 9-13, 2012, TC21-O-9, paper 724.

12. A. J. Kearsley, Optimality Conditions for a Hierarchical Control Problem Governed by a PDE, in Proceedings of the 2nd International Conference on Instrumentation Control and Automation
(ICA), Bandung, Indonesia November 15-17, 2011.

13. D. R. Kuhn, J. Higdon, J. Lawrence, R. Kacker

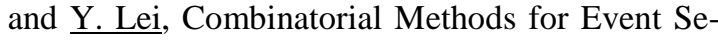
quence Testing, in Proceedings of the Fifth IEEE International Conference on Software Testing, Verification and Validation, First International Workshop on Combinatorial Testing (IWCT) Montreal Canada, April 17-21, 2012 pp. 601-609.

14. P. S. Kuo, J. S. Pelc, O. Slattery, L. Ma, M. M. Fejer and $X$. Tang, Dual-channel, Single-photon Upconversion Detector at $1300 \mathrm{~nm}$, in Nonlinear Photonics, OSA Technical Digest, Optical Society of America, 2012, NM3C.6.

15. P. S. Kuo, J. S. Pelc, O. Slattery, M. M. Fejer and $\underline{X}$. Tang, Dual-channel, Single-photon Upconversion Detector Near $1300 \mathrm{~nm}$, in Proceedings of SPIE 8518 (2012), 85180U1-12.

16. P. S. Kuo, J. S. Pelc, O. Slattery, M. M. Fejer and $\underline{X}$. Tang, Photon Temporal Correlations Measured Using a Dual-Channel Upconversion Detector, in Frontiers in Optics Conference, OSA Technical Digest, Optical Society of America, 2012, FTh4B.1.

17. Y.-K. Liu, Universal Low-rank Matrix Recovery from Pauli Measurements, in Advances in Neural Information Processing Systems (NIPS), 2011, 1638-1646.

18. F. Maggioni, M. Bertocchi, E. Allevi, F. A. Potra and S. W. Wallace, Stochastic second-order cone programming in mobile ad-hoc networks: sensitivity to input parameters, Stochastic Programming, Applications in Finance, Energy and Logistics $(\mathrm{H}$. I. Gassmann, S. W. Wallace, W. T. Ziemba eds), World Scientific, 2012, 20 pages.

19. H. Mahboubi, W. Masoudimansour, A. G. Aghda,

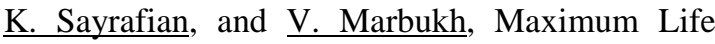
Span Strategy for Target Tracking in Mobile Sensor Networks, in Proceedings of 2012 American Control Conference, Montreal, Canada, June 2012, 5096-5101.

20. H. Mahboubi, W. Masoudimansour, A. G. Agh-

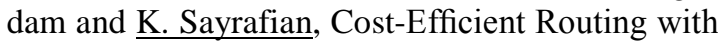
Controlled Node Mobility in Sensor Networks, in Proceedings of 2011 IEEE Multiconference on Systems \& Control, Denver, USA, September 2011, 1238-1243.

21. H. Mahboubi, K. Moezzi, A. G. Aghdam, K. Sayrafian and V. Marbukh, Distributed Deployment Algorithms for Improved Coverage in Mobile Sensor Networks, in Proceedings of 2011 IEEE 
Multiconference on Systems \& Control, Denver, USA, September 2011, 1244-1249.

22. H. Mahboubi, J. Habibi, A. G. Aghdam and $\underline{K}$.

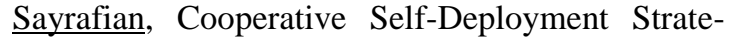
gies in a Mobile Sensor Network with Prioritized Coverage Plan, in Proceedings of 2011 IEEE Global Communications Conference (GLOBECOM), Houston, USA, December 2011.

23. V. Marbukh, Risks/benefits of Selfish Network Operations \& Management in Dynamic Environment, 13th IEEE/IFIP Network Operations and Management Symposium (NOMS 2012), Maui, HI, 2012.

24. V. Marbukh, Towards Modeling Effect of Packetlevel TCP Dynamics on Internet Operations and Management, 13th IEEE/IFIP Network Operations and Management Symposium (NOMS 2012), Maui, HI, 2012.

25. Ion Matei, A. Gueye and John S. Baras. Flow Control in Time-Varying, Random Supply Chain Networks. in $20^{\text {th }}$ International Symposium on Mathematical Theory of Networks and Systems (MTNS), Melbourne, Australia July 9-13, 2012.

26. A. Nakassis and A. Mink, LDPC error correction in the context of Quantum Key Distribution, in Proceedings of SPIE: Defense Security \& Sensing 8400 (2012), 840009-1.

27. A. P. Peskin, D. J. Hoeppner, C. H. Stuelten, Segmentation and Cell Tracking of Breast Cancer Cells, in Proceedings of the 7th International Symposium on Visual Computing (G. Bebis et al., eds), Part I, Lecture Notes in Computer Science 6938 (2011), 381-391.

28. A. P. Peskin, A. Dima and G. Saiprasad, An Automated Method for Locating Phantom Nodules in Anthropomorphic Thoracic Phantom CT Studies, in Proceedings of the International Conference on Image Processing and Computer Vision, Las Vegas, July 16-19, 2012.

29. S. Ressler, Slides, Filters and Mobile Oh My! (Demonstrations of HTML and Declarative 3D Integration), in Proceedings of the 1st International Workshop on Declarative 3D for the Web Architecture, Lyon, France, April 17, 2012.

30. S. Ressler, Web-based Declarative 3D Graphics for Anthropometry Visualization and Education, in The Graphical Web, Zurich, Switzerland, September 13, 2012.

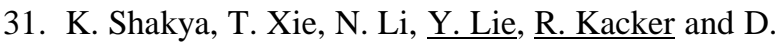
R. Kuhn, Isolating Failure-Inducing Combinations in Combinatorial Testing uses Test Augmentation and Classification, in Proceedings of the Fifth
IEEE International Conference on Software Testing, Verification and Validation, First International Workshop on Combinatorial Testing (IWCT) Montreal Canada, April 17-21, 2012, 620-623.

32. E. Simiu, F. A. Potra and D. Duthinh, Methodological and probabilistic issues in multi-hazard engineering design, in Applications of Statistics and Probability in Civil Engineering (Michael Faber, Jochen Koehler and Kazuyoshi Nishijima, Editors), CRC Press, ISBN: 978-0-415-66986-3, Leiden, The Netherlands, 2011, 836-839.

33. O. Slattery, Paulina Kuo, Yong-Su Kim, L. Ma and Xiao Tang, Narrowed Bandwidth SPDC Correlated Photon Source using Volume Bragg Grating, in Proceedings of SPIE 8518 (2012), 85180Y1-10.

34. J. C. Wu, A. F. Martin, C. S. Greenberg and R. Kacker, Data Dependency on Measurement Uncertainties in Speaker Recognition Evaluation, in Active and Passive Signatures III, Proceedings of the SPIE 8382 (2012), 83820D, 12 pages.

35. W. Yang and K. Sayrafian, Radiation Pattern of an UWB Wearable Antenna: A Preliminary Study, in Proceedings of the $7^{\text {th }}$ International Conference on Body Area Networking (BodyNets), Oslo, Norway, September 24-26, 2012

36. D. H. Yeo, F. T. Lombardo, D. K. Banerjee, E. J. Letvin, E. Simiu, M. L. Levitan and F. A. Potra, NIST advances in computer-aided and computational methods in wind engineering, in Proceedings of the ATC-SEI Advances in Hurricane Engineering Conference, Miami, FL, 2012 (12 pages).

37. L. Yu, Yu Lei, R. Kacker, D. R. Kuhn and J. Lawrence, Efficient Algorithms for T-Way Test Sequence Generation, in Proceedings of the 17-th IEEE International Conference on Engineering of Complex Computer Systems (ICECCS), Paris, France, July 18-20 , 2012, 220-229.

\section{Sponsored Research}

1. P. Cheng, L. Wang, S. Jajodia and A. Singhal, Aggregating CVSS Base Scores for SemanticsRich Network Security Metrics, in Proceedings of the $31^{\text {st }}$ IEEE International Conference on Reliable and Distributed Systems, Irvine, October 2012.

2. R. Zhuag, S. Zhang, S. Deloach, X. Ou, and A. Singhal, Simulation-based Approaches to Studying Effectiveness of Moving Target Network Defense, in Proceedings of the National Symposium on Moving Target Defense, Annapolis, MD, June 2012. 


\section{Technical Magazine Articles}

1. I. Beichl, Just Try, Computing in Science \& Engineering 13:6 (2011), 4.

2. I. Beichl, Of Art and CS Education, Computing in Science \& Engineering 14:1 (2012), 4.

3. I. Beichl, Is My Car Smarter than My Cat?, Computing in Science \& Engineering 14:3 (2012), 4.

4. I. Beichl, Counting on Today's Students, Computing in Science \& Engineering 14:5 (2012), 4.

5. I. Beichl, Hail and Farewell, Computing in Science \& Engineering 14:6 (2012), 4.

6. D. R. Kuhn. J. Higdon, J. Lawrence, R. Kacker and Y. Lei, Efficient Methods for Interoperability Testing Using Event Sequences, CrossTalk : the Journal of Defense Software Engineering (July/August 2012), 15-18.

7. D. P. O'Leary and B. W. Rust, Light a Single Candle: Studying Supernovae, Computing in Science and Engineering 14:2 (March/April 2012), 90-96.

\section{Technical Reports}

1. R. F. Boisvert, Applied and Computational Mathematics Division, Summary of Activities for Fiscal Year 2011, NISTIR 7856, May 2012.

2. A. S. Carasso, Alternative Methods of Latent Fingerprint Enhancement and Metrics for Comparing Them, NISTIR 7910, January 2013.

3. W. F. Mitchell and M. A. McClain, A Comparison of $h p$-Adaptive Strategies for Elliptic Partial Differential Equations (long version), NISTIR 7824, October 2011.

4. E. Simiu and F. A. Potra, Technical Basis for Regulatory Guidance on Design-Basis HurricaneBorne Missile Speeds for Nuclear Power Plants (NUREG/CR-7004), Office of Nuclear Regulatory Research, U.S. Nuclear Regulatory Commission, Washington, DC 20555-0001, November 2011, (110 pages).

5. J. C. Wu, M. Halter, R. Kacker and J. Elliott, A New Measure in Cell Image Segmentation Data Analysis, NISTIR 7871, July 2012.

6. J. C. Wu, A. F. Martin, C. S. Greenberg and R. Kacker and V. Stanford, Significance Test in Speaker Recognition Data Analysis with Data Dependency” NISTIR 7884, October 2012.

\section{Accepted}

1. A. Anandkumar, D. P. Foster, D. Hsu, S. M. Kakade and Y.-K. Liu, Two SVDs Suffice: Spectral Decompositions for Probabilistic Topic Modeling and Latent Dirichlet Allocation, in Advances in Neural Information Processing Systems (NIPS), 2012.

2. F. Backeljauw, S. Becuwe, A. Cuyt, J. Van Deun and D. W. Lozier, Validated Evaluation of Special Mathematical Functions, Science of Computer Programming.

3. H. S. Cohl, On a Generalization of the Generating Function for Gegenbauer Polynomials, Integral Transforms and Special Functions.

4. A. Gueye, V. Marbukh and J. C. Walrand. Towards a Metric for Communication Network Vulnerability to Attacks: A Game Theoretic Approach, $8^{\text {th }}$ International Conference on Information Warfare and Security ICIW-2013 Regis University, Denver, CO, March 25-26, 2013.

5. D. G. Harris, I. Beichl and F. Sullivan, Fast Sequential Importance Sampling to Estimate the Graph Reliability Polynomial, Algorithmica.

6. R. L. Jacobsen, R. D. Johnson III, K. K. Irikura and R. Kacker, Anharmonic Vibrational Frequency Calculations are not Worthwhile for Small Basis Sets, Journal of Chemical Theory and Computation

7. A. J. Kearsley, Constrained Hierarchical Control of a Diffusion Equation, International Journal of Applied Mathematics and Statistics.

8. H. Mahboubi, K. Moezzi, A. G. Aghdam, K. Say$\underline{\text { rafian and V. Marbukh, Distributed Deployment }}$ Algorithms for Improved Coverage in a Network of Wireless Mobile Sensors," IEEE Transactions on Industrial Informatics.

9. H. Mahboubi, J. Habibi, A. G. Aghdam and K. Sayrafian, Distributed Deployment Strategies for Efficient Coverage in a Network of Mobile Sensors with Prioritized Sensing Field, IEEE Transactions on Industrial Informatics.

10. W. Masoudimansour, H. Mahboubi, A. G. Aghdam and K. Sayrafian, Maximum Lifetime Strategy for Target Monitoring in Mobile Sensor Networks in Presence of Obstacles, Proceedings of $51^{\text {st }}$ IEEE Conference on Decision and Control, Maui, USA, December 2012. 
11. A. Meier, B. Eastin and E. Knill, Magic-State Distillation with the Four-Qubit Code, Quantum Information and Computation.

12. A. K. Nurse, G. B. McFadden and S. R. Coriell, Bubble Motion and Size Variation during Thermal Migration with Phase Change, Physics of Fluids.

13. D. P. O'Leary and B. W. Rust, Variable Projection for Nonlinear Least Squares Problems, Computational Optimization and Applications.

14. R. Pozo, Efficient Q-matrix Computation for the Visualization of Complex Networks, in Proceedings of the 8th International Conference on Signal Image Technology \& Internet Based Systems, 2012.

15. J. C. Wu, A. F. Martin and R. Kacker, Bootstrap Variability Studies in ROC Analysis on Large Datasets, Communications in Statistics.

16. W. Yang and K. Sayrafian, Theoretical Analysis and Modeling of Link Adaptation Schemes in Body Area Networks, Proceedings of the 3th International Conference on Mobile Health (MobiHealth), November 2012.

\section{In Review}

1. B. K. Alpert, R. D. Horansky, D. A. Bennett, W. B. Doriese, J. W. Fowler, A. Hoover, M. W. Rabin, J. N. Ullom, Filters for High Rate Pulse Processing.

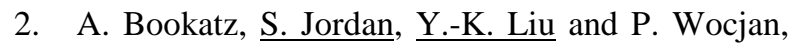
Testing Quantum Expanders is Co-QMAcomplete.

3. A. S. Carasso, Hazardous Continuation Backward in Time in Nonlinear Parabolic Equations and an Experiment in Deblurring Nonlinearly Blurred Imagery.

4. J. Chalfoun, A. Dima, M. Kociolek, M. Halter, A. Cardone, A. Peskin, P. Bajcsy and M. Brady, Segmenting Time-lapse Phase Contrast Images of Adjacent NIH 3T3 Cells.

5. H. S. Cohl and C. MacKenzie, Generalizations and Simplifications of Generating Functions for Jacobi, Gegenbauer, Chebyshev and Legendre Polynomials with Definite Integrals, arXiv:1210.0039.

6. H. S. Cohl, Fourier, Gegenbauer and Jacobi Expansions for a Power-law Fundamental Solution of the Polyharmonic Equation and Polyspherical Addition Theorems, arXiv:1209.6047.
7. H. S. Cohl, Fourier Expansions for Logarithmic Fundamental Solutions of the Polyharmonic Equation, arXiv:1202.1811.

8. H. S. Cohl and H. Volkmer, Separation of Variables in an Asymmetric Cyclidic Coordinate System, arXiv:1301.3559.

9. H.S. Cohl, Antiderivatives and Integral Representations of Associated Legendre Functions with $\mu= \pm v$, arXiv:1301.3556.

10. D. Cotrell, A. Lott and G. McFadden, Boundary Slip Effects on the Stability of Spiral Poiseuille Flow.

11. R. Chávez, K. Sayrafian, A. Khalegi, K. Takizawa, J. Wang, I. Balasingham and H. Li, Radio Propagation Models for IEEE 802.15.6 Standardization of Implant Communication in Body Area Networks.

12. E. Cobanera, G. Ortiz and E. Knill, The NonAbelian Duality Problem.

13. J. T. Fong, N.A. Heckert, J. J. Filliben and W.F. Guthrie, A Bayesian and Quantitative Nondestructive Evaluation (QNDE) Approach to Estimating Remaining Life of Pressure Vessels \& Piping.

14. J. T. Fong, N.A. Heckert and J. J. Filliben, Composites Failure Criteria, Uncertainty Propagation and Estimation of the A-basis and B-basis Design Allowables.

15. J. T. Fong, C. A. Cimini, Jr, J. D. D. Melo, N. A. Heckert and J. J. Filliben, Theoretical Failure Envelopes of Open Hole Composite Laminates with A- and B-basis Allowables Estimated from Smooth Specimen Properties.

16. S. W. Freiman and J. T. Fong, Determining Uncertainties in Failure Time Predictions for Brittle Materials.

17. A. Gueye, Aron Lazska, Jean C. Walrand and V. Anantharan, A Polyhedral-Based Analysis of Nash Equilibria of Quasi-Zero-Sum Games and its Applications to Communication Network Security.

18. C. Hagwood, J. Bernal, M. Halter, J. Elliott, T. Brennan, Testing Equality of Cell Populations based on Shape and Geodesic Distances.

19. W. F. Mitchell and M. A. McClain, A Comparison of $h p$-Adaptive Strategies for Elliptic Partial Differential Equations.

20. W. F. Mitchell, A Collection of 2D Elliptic Problems for Testing Adaptive Algorithms.

21. E. Moseman, I. Beichl and F. Sullivan, Two Algorithms to Approximate Multivariate Network Reliability. 
22. M. Ohliger, V. Nesme, D. Gross, Y.-K. Liu and J. Eisert, Continuous-variable Quantum Compressed Sensing.

23. K. Pahlavan, G. Bao, Y. Ye, S. Makarov, U. Khan, P. Swar, D. Cave, A. Karellas, P. Krishnamurthy and $\underline{\mathrm{K}}$. Sayrafian, RF Localization for Wireless Video Capsule Endoscopy.

\section{Sponsored Research}

24. J. D. Hobby and G. H. Tucci, Traffic Analysis in Random Delaunay Tessellations and Other Graphs, arXiv: 1203.4863.

25. O. Narayan, I. Saniee and G. H. Tucci, Lack of Hyperbolicity in Asymptotic Erdos-Renyi Sparse Random Graphs, arXiv: 1009.5700v2.

26. G. H. Tucci, Random Regular Graphs are not Asymptotically Gromov Hyperbolic, arXiv: 1203.5069.

27. L. Wang, A. Singhal, S. Jajodia and S. Noel, A Network Security Metric for Measuring and Improving Computer Networks Resistance to Unknown Vulnerabilities.

\section{Presentations}

\section{Invited Talks}

1. I. Beichl, "Linear Algebra and Sequential Importance Sampling for Network Reliability," Winter Simulation Conference, Phoenix, AZ, December 13, 2011.

2. R. F. Boisvert, "Fair Access: Society Publishing," Perspectives Workshop on the Publication Culture in Computing Research, Liebnitz Center for Informatics, Schloss Dagstuhl, Germany, November 7, 2012.

3. R. F. Boisvert, "Reproducibility at ACM," Panel Discussion on Journal Policies/Experiences, Workshop on Reproducibility in Computational and Experimental Mathematics, Institute for Computational and Experimental mathematics, Brown University, Providence, RI, December 11, 2012.

4. T. J. Burns, "Transient Convection-diffusion Modelling of Peak Temperature in Orthogonal Cutting," 23rd International Congress of Theoretical and Applied Mechanics (ICTAM2012), Beijing, China, August 24, 2012.

5. A. S. Carasso, "Unexplored Territory in Nonstandard Parabolic Equations and their Application," Joint Numerical Analysis/Norbert Wiener Center Seminar, Mathematics Department,
University of Maryland, College Park MD, December 6, 2011.

6. A. S. Carasso, "Non-standard Parabolic Equations and Image Reconstruction," AMS-SIAM Minisymposium on Applied, Computational and Discrete Mathematics at National Laboratories and Federal Research Agencies, Joint Mathematics Meetings, Boston, MA, January 4-7, 2012.

7. B. Cloteaux, "Affiliation Networks," Old Dominion University Computer Science Seminar, Norfolk, VA, October 28, 2011.

8. H. S. Cohl, "The Fourier and Gegenbauer Analysis of Fundamental Solutions for the Laplace Equation on Riemannian Spaces of Constant Curvature," Department of Mathematical Sciences, University of Wisconsin--Milwaukee, Milwaukee, Wisconsin, October 7, 2011.

9. H. S. Cohl, "Fourier, Gegenbauer and Jacobi Expansions related to a Fundamental Solution of the Polyharmonic Equation," Department of Mathematical Sciences, University of Wisconsin-Milwaukee, Milwaukee, Wisconsin, October 7, 2011.

10. H. S. Cohl, "Addition Theorems in Separable Coordinate Systems for Fundamental Solutions," Department of Mathematical Sciences, University of Wisconsin--Milwaukee, Milwaukee, Wisconsin, March 2, 2012.

11. H. S. Cohl, "Fourier, Gegenbauer and Jacobi Expansions," Department of Mathematics and Statistics, American University, Washington DC, March 20, 2012.

12. H. S. Cohl, "The Fourier and Gegenbauer Analysis of Fundamental Solutions for Laplace's Equation on Riemannian Spaces of Constant Curvature," Department of Mathematics, Louisiana State University, Baton Rouge, Louisiana, August 14, 2012.

13. H. S. Cohl, "Simplifications and Generalizations of Generating Functions for Hypergeometric Orthogonal Polynomials with Definite Integrals," Department of Mathematical Sciences, University of Wisconsin--Milwaukee, Milwaukee, Wisconsin, September 28, 2012.

14. H. S. Cohl, "Generalizations of Generating Functions for Hypergeometric Orthogonal Polynomials," The Norbert Weiner Center for Harmonic Analysis, University of Maryland, College Park, Maryland, October 16, 2012.

15. H. S. Cohl, "Expansions for the Iterated Poisson Equation on $d$-dimensional Euclidean Space," Department of Mathematical Sciences, George 
Mason University, Fairfax, Virginia December 7, 2012.

16. A. Dienstfrey, Panelist, American Society of Mechanical Engineers, 2012 Verification and Validation Symposium, Las Vegas, NV, May 2-4, 2012.

17. A. Dienstfrey, "Computation, Measurement, and Standards," NAFEMS North America Conference, Herndon, VA, September 10-12, 2012.

18. G. Dogan, "An Iterative Shape Reconstruction Algorithm for Inverse Problems," 6th International Conference on Inverse Problems: Modeling \& Simulation, Antalya, Turkey, May 21-26, 2012.

19. G. Dogan, "Variational Shape Optimization for Image Segmentation," Fall Eastern American Mathematical Society Sectional Meeting, Rochester Institute of Technology September 2223, 2012.

20. M. J. Donahue, "Numerical Methods in Micromagnetics for Fast, Accurate Computation of Demagnetization Kernels with Periodic Boundaries," IMA/MCIM Industrial Problems Seminar at the University of Minnesota, Minneapolis, Minnesota, February 24, 2012.

21. M. J. Donahue, "Numerical Methods in Micromagnetics for Fast, Accurate Computation of Demagnetization Kernels with Periodic Boundaries," International Workshop on Advanced Micromagnetics (IWAM) at the Center for Magnetic Recording Research, University of California, San Diego, May 21-22, 2012.

22. M. J. Donahue, "Recent Advances in Micromagnetics," IEEE Magnetics Society, University of Colorado, Boulder, Colorado, August 2, 2012.

23. J. T. Fong, "Design of and Modern Statistics for Improving Reliability of Composite Materials," International JEC/Asia Composite Materials Design Forum, Singapore, October 20, 2011. [Invited Keynote Lecture]

24. J. T. Fong, "Design of Experiments and Modern Statistics for Modeling and Failure Prediction of Full-Scale Structures," Department of Mechanical Engineering, National University of Singapore, Singapore, October 21, 2011.

25. J. T. Fong, "Design of Experiments and Modern Statistics for Modeling and Failure Prediction of Full-Scale Structures," Department of Mechanical Engineering, Nanyang University, Singapore, October 24, 2011.

26. J. T. Fong, "Uncertainty Estimation and Failure
Prediction of Full-Scale Structures using a MultiScale Approach," School of Mechanical Engineering, East China University of Science and Technology, Shanghai, China, December 9, 2011.

27. J. T. Fong, "Modern Statistics, Exploratory Data Analysis and Design of Experiments for Improving Reliability of Aging Engineering Structures," Department of Mathematics, Ningpo University, Ningpo, China, December 12, 2011.

28. J. T. Fong, "Modern Statistics, Data Analysis and Design of Experiments for Improving Reliability of Aging Components and Structures," Department of Mechanical Engineering, City University of Hong Kong, Hong Kong, December 14, 2011.

29. J. T. Fong, "Risk-Informed Inspection and OnLine Monitoring of High-Consequence Aging Structures and Power Plants," Department of Mechanical Engineering, City University of Hong Kong, Hong Kong, December 15, 2011.

30. J. T. Fong, "Engineering Statistics and Reliability Analysis of Composite Structures," $6^{\text {th }}$ Composite Design Workshop, Department of Aeronautics and Astronautics, Stanford University, Stanford, CA, Jan. 24, 2012.

31. J. T. Fong, "Composites Failure Criteria with Uncertainty Quantification for A- and B-Design Allowables," JEC/Europe Composite Materials Design Forum, Paris, France, Mar. 27, 2012.

32. J. T. Fong, "Modern Statistics, Data Analysis and Design of Experiments for Improving Reliability of Aging Engineering Structures," National Physical Laboratory, Teddington, United Kingdom, April 3, 2012. [Distinguished Science Lecture]

33. J. T. Fong, "Modern Statistics, Uncertainty Propagation and Design of Experiments for Improving Reliability of Aging Engineering Structures," Center for Nondestructive Evaluation, Iowa State University, Ames, IA, April 23, 2012.

34. J. T. Fong, "Multi-scale Failure Simulation, Uncertainty Propagation Theory and NDE-based Health Monitoring of Aging Structures," Department of Civil and Environmental Engineering, University of South Carolina, Columbia, SC, May 4, 2012

35. J. T. Fong, "On the Relative Value of Material Property Databases with, or, without an Expression of Uncertainty," e-Ceramics Workshop, Department of Defense, Arlington, VA, June 4, 2012.

36. J. T. Fong, "Multi-scale Modeling with 
Uncertainty Quantification for Reliability of HighConsequence Aging Structures," Materials Reliability Division, National Institute of Standards \& Technology, Boulder, CO, July 20, 2012.

37. J. T. Fong, "Engineering Statistics and Reliability Analysis of Composite Structures," $7^{\text {h }}$ Composite Design Workshop, Department of Aeronautics and Astronautics, Stanford University, Stanford, CA, Aug. 22, 2012.

38. J. T. Fong, "Fatigue Life Estimation of Aging Nuclear Components," Electric Power Research Institute, Charlotte, NC, September 5, 2012.

39. J. T. Fong, "Quantitative Nondestructive Evaluation (QNDE) and Stochastic Modeling of Fatigue of Aging Structures," Department of Mechanical Engineering, Yokohama National University, Yokohama, Japan, October 15, 2012.

40. J. T. Fong, "Quantitative Technology of Nondestructive Evaluation (QT-NDE): Key to Innovative Manufacturing and Aging Equipment Management," School of Mechanical Engineering, East China University of Science and Technology, Shanghai, China, October 19, 2012.

41. J. T. Fong, "Quantitative Technology of Nondestructive Evaluation (QT-NDE): Key to Innovative Manufacturing and Aging Equipment Management," China Special Equipment Inspection and Research Institute, Beijing, China, October 22, 2012.

42. J. T. Fong, "Quantitative Technology of Nondestructive Evaluation (QT-NDE): Key to Innovative Manufacturing and Aging Equipment Management," Department of Mechanical Engineering, Beijing Institute of Technology, Beijing, China, October 23, 2012.

43. J. T. Fong, "Quantitative Technology of Nondestructive Evaluation (QT-NDE): Key to Innovative Manufacturing and Aging Equipment Management," Department of Materials Science, Dalian University of Technology, Dalian, China, October 25, 2012.

44. J. T. Fong, "Quantitative Technology of Nondestructive Evaluation (QT-NDE): Key to Innovative Manufacturing and Aging Equipment Management," Department of Mechanical Engineering, China University of Petroleum, Qingdao, China, October 29, 2012.

45. J. T. Fong, "A Stochastic and Local Theory of Fatigue for Aging Pressure Vessels and Piping," $3^{\text {rd }}$ International Symposium on Structural Integrity (ISSI), October 31 - Nov. 4, 2012,
Shandong University, Jinan, China, November 1, 2012. [Keynote Lecture]

46. W. L. George, N. S. Martys and J. E. Terrill, "Computational Rheology for Cement and Concrete," Workshop on High-Performance Computing for Industry, Rensselaer Polytechnic Institute, October 26-28, 2011.

47. W. L. George, N. S. Martys, S. G. Satterfield, J. E. Terrill, "Modeling the Flow of Cement and Concrete in a Rheometer," Argonne Leadership Computing Facility Seminar Series, Argonne National Laboratory, May 21, 2012.

48. W. L. George, N. S. Martys, S. G. Satterfield, M. Olano and J. E. Terrill, "Advancing the Materials Science of Concrete with Supercomputers," Argonne National Laboratory Exhibit, SC Conference, Seattle WA, November 2011.

49. S. Glancy, "Progress Towards Quantum Teleportation of Traveling Microwaves," Workshop-School in Quantum Computation and Information, Fortaleza, Brazil, October 11, 2012.

50. A. Gueye, "On the Value of Communication Network (Security)," Institute for Systems Research, University of Maryland, November 4, 2011.

51. A, Gueye, "On the Value of Communication Network Security," MURI workshops (MLMR, ADCN, MAASCOM), University of California, Berkeley, October 27, 2011.

52. S. Jordan, "Quantum Algorithms for Simulating Quantum Field Theories," NASA Future Technologies Conference, Mountain View, CA, January 21, 2012.

53. S. Jordan, "Quantum Algorithms for Simulating Quantum Field Theories," C. N. Yang Institute Seminar, SUNY Sony Brook, NY, March 8, 2012.

54. S. Jordan, "Quantum Algorithms for Simulating Quantum Field Theories," Capital Area Theory Seminar, University of Maryland, College Park, MD, April 6, 2012.

55. R. Kacker, "Introduction to Combinatorial Testing," Department of Mathematics and Statistics, University of Maryland Baltimore County, December 2, 2011.

56. R. Kacker, "Combinatorial Testing for Software," Infosys Education and Research (E\&R) Unit Winter School on Software Validation, Verification and Reliability, Bangalore, India, December 16, 2011.

57. R. Kacker, "Combinatorial Testing for Software," Philips Research, Bangalore, India, Dec. 19, 2011. 
58. R. Kacker, "Combinatorial Testing for Software," IBM-India, Bangalore India, December 19, 2011

59. R. Kacker, "Evolution of Combinatorial Testing for Software from Design of Experiments," International Conference on Quality and Reliability Engineering (ICQRE-2011), Indian Statistical Institute, Bangalore India, December 20, 2011.

60. R. Kacker, "Uncertainty Requirements in the Contract SOW for GOES-R and Implementation of VIM3 and GUM," CALCON 2012 Technical Conference Utah State University, August 27, 2012.

61. A. J. Kearsley, "Stochastic Derivative Approximations in Calibrating Mass Spectrometers," Universitas Bakrie, Indonesia, September 13, 2012.

62. A. J. Kearsley, "Optimal Finite Difference Calculations for Maximizing Signal to Noise," Jakarta State University, September 21, 2012.

63. A. J. Kearsley, "Optimality Conditions for a Hierarchical Control Problem Governed by a PDE," 2nd International IEEE Conference on Instrumentation Control and Automation (ICA), Bandung, Indonesia, November 15-17, 2011.

64. A. J. Kearsley, "Survey of Mathematics Applications at NIST," Putera Sampoerna School of Education, Jakarta, Indonesia, September 19, 2012.

65. A. J. Kearsley, "Optimal Library Searching through Multidimensional Scaling," University of Calgary, June 19, 2012.

66. A. J. Kearsley, "Instrument Tuning using a Stochastic Gradient Approximation," University of Calgary, June 24, 2012.

67. Y.-K. Liu, "Quantum Algorithms for Testing Graph Expansion and Bipartiteness," Computer Science Department, University of Maryland, March 9, 2012.

68. Y.-K. Liu, "Universal Low-rank Matrix Recovery from Pauli Measurements," Perimeter Institute, Waterloo, Canada, April 4, 2012.

69. Y.-K. Liu, "Universal Low-rank Matrix Recovery from Pauli Measurements," Microsoft Research New England, Boston, April 10, 2012.

70. Y.-K. Liu, "Matrix Completion," Computer Science Department, George Washington University, April 19, 2012.

71. Y.-K. Liu, "Universal Low-rank Matrix Recovery from Pauli Measurements," Institute for Defense
Analysis Center for Computing Sciences, Bowie, MD, July 27, 2012.

72. Y.-K. Liu, "Some Uses of Quantum Information in Machine Learning and Cryptography," JQI Quantum Information Science Workshop, College Park, MD, September 28, 2012.

73. D. W. Lozier, "DLMF Tables: A New Source of Data for Mathematical Software Developers," Workshop on Numerical Software: Design, Analysis and Verification, University of Cantabria, Santander, Spain, July 4-6, 2012.

74. V. Marbukh, "Some Personal Observations on System Complexity," Network Complexity Research Group (NCRG) Meeting, Internet Research Task Force (IRTF), Paris, France, 2012.

75. N. Martys and W. George, "Computational Modeling of Suspensions: Application to Cement Based Materials," Monte Verita Conference on Microstructure, Setting and Aging of Cement: From Soft Matter Physics to Sustainable Materials, Monte Verita, Ascona, Switzerland, August 12-16, 2012.

76. G. B. McFadden, "Richard Tapia's Influence as an Undergraduate Teacher and Mentor," A Celebration of Richard Tapia Receiving the National Medal of Science, Rice University, March 3, 2012.

77. G. B. McFadden, "Bubble Motion and Size Variation during Thermal Migration with Phase Change," Department of Mathematical Sciences, University of Delaware, April 24, 2012.

78. W. F. Mitchell, "Comparison of $h p$-Adaptive Finite Element Strategies." George Washington University, Washington, DC, March 22, 2012.

79. D. P. O'Leary, "Image Restoration from a Machine Learning Perspective," 2012 Cray Distinguished Speaker, Computer Science Department, University of Minnesota, April, 2012.

80. A. Peskin, A. Dima and G. Saiprasad, "An Automated Method for Locating Phantom Nodules in Anthropomorphic Thoracic Phantom CT Studies," American Statistical Association Spring Meeting, Colorado-Wyoming Chapter, April 20, 2012,

81. Sandy Ressler, "3D Computer Graphics on the World Wide Web," Stockton Computer Society, Galloway NJ, March 29, 2012. [ACM Distinguished Lecture]

82. B. Saunders, "Interactive 3D Visualizations of Complex Function Data in the NIST Digital Library of Mathematical Functions," SIAM Math 
Awareness Conference, Old Dominion University, Norfolk, VA, March 24, 2012.

83. B. Saunders, "Constructing Interactive 3D Visualizations for the NIST Digital Library of Mathematical Functions," SIAM Minisymposium on Applied, Computational and Discrete Mathematics at National Laboratories and Federal Research Agencies, AMS-MAA-SIAM Joint Mathematics Meetings, Boston, MA, January 5, 2012.

84. B. Saunders, Panelist, Conversation on NonAcademic Employment, Joint Mathematics Meetings, Boston, MA, January 5, 2012.

85. K. Sayrafian, "Body Area Networks: Standardization Activities and Technology Challenges," Centre for Communication Systems Research, University of Surrey, Guildford, UK, October 11, 2012.

86. K. Sayrafian, "RF Propagation in Body Area Networks," Workshop on Medical Communication Systems (ACM MedCOMM), Helsinki, Finland, August 13, 2012

87. K. Sayrafian, "Personalized Health: An ICT Perspective," COST Strategic Conference on Personalised Medicine (PEMED), Larnaca, Cyprus, June 18-22, 2012.

88. K. Sayrafian, "Opportunities and Challenges in Body Area Networks," Department of Electrical \& Computer Engineering, Worcester Polytechnic Institute, Worcester, MA, February 23, 2012.

89. K. Sayrafian, Panelist, Health and Medical CPS Panel, National Science Foundation Cyber Physical Systems PI Meeting \& Conference, October 4, 2012.

90. K. Sayrafian, "The Role of Standardization in Healthcare," mHealth Summit, National Harbor, MD, December 5, 2011.

91. N. Streib, "An Application of Graph Theory in Theoretical Physics," University, Discrete Mathematics Seminar, Virginia Commonwealth Richmond, VA, December 4, 2012.

92. Xiao Tang, "Single Photon Frequency Conversion and its Applications in Quantum Information Research," Department of Electrical Engineering, Washington University, St. Louis, April 13, 2012.

93. Xiao Tang, "Dual Channel Upconversion Device for NIR Single-photon Detection," The 21th International Laser Physics Workshop (LPHYS'12), University of Calgary, Calgary, Canada, July 23 - 27, 2012.

\section{Conference Presentations}

1. P. Bajcsy, J. Chalfoun, A. Cardone, A. Peskin, M. Halter, K. Bhadriraju, J. J. Filliben, J. T. Elliott, M. Kociolek, M. C. Brady and A. L. Plant, "Computational Science for Biological Metrology," Poster, 5th Advances in Stem Cell Discovery and Development Conference, San Diego, October 19-21, 2011.

2. J. W. Bullard, D. P. Bentz, E. J. Garboczi, S.G. Satterfield, P.E. Stutzman, L. Valenti and G. Artioli, "Simulating Solution Chemistry and Phase Evolution in Early-age Cement Pastes," American Concrete Institute Spring 2012 Convention, March 18-22, Dallas, Texas.

3. T. J. Burns, "NIST and the MGI," IMA Special Workshop: Mathematics and the Materials Genome Initiative, University of Minnesota, Minneapolis, MN, September 13, 2012.

4. B. Cloteaux, "Extracting Hierarchies with Overlapping Structure from Network Data", Winter Simulation Conference, Phoenix, AZ, December 13, 2011.

5. B. Cloteaux, "Extracting Affiliations from Networks", NIST-Bell Labs Workshop on LargeScale Complex Networks, Gaithersburg, MD, June 8, 2012.

6. H. S. Cohl, "Super Expansions and Definite Integrals for Jacobi, Gegenbauer, Legendre and Chebyshev Polynomials," International Symposium on Orthogonal Polynomials and Special Functions -- A Complex Analytic Perspective, Copenhagen, Denmark, June 14, 2012.

7. A. Dienstfrey, "A Metrology Infrastructure for Scientific Computing," National Radio Science Meeting, University of Colorado, Boulder, CO, Jan 4-7, 2012

8. G. Dogan, "Fast Iterative Algorithm for Shape Reconstruction in Image Processing," Copper Mountain Conference on Iterative Methods, Copper Mountain, CO, March 25-30, 2012.

9. G. Dogan, "An Algorithm for Shape Detection in Inverse Problems," SIAM Conference on Imaging Sciences, Philadelphia, PA, May 20-22, 2012.

10. G. Dogan, "Energy-based Segmentation Methods for Micrograph Analysis," International Conference on 3D Materials Science, Seven Springs, PA, July 8-12, 2012.

11. G. Dogan, "Variational Algorithms for Fast and Robust Shape Reconstruction in Imaging," European Congress on Computational Methods in 
Applied Sciences and Engineering, Vienna, Austria, September 10-14, 2012.

12. M. J. Donahue, "Fast, Accurate Computation of the Demagnetization Tensor for Periodic Boundaries," Intermag 2012, Vancouver, Canada, May 8, 2012.

13. B. Eastin, "Simulating Concordant Computations," Quantum Discord Workshop, Singapore, January 10, 2012.

14. J. T. Fong, "Uncertainty Estimation of Time-toFailure of a Full-Scale Structure on Fire using a Stochastic and Multi-Scale Approach," $48^{\text {th }}$ Annual Meeting of Society of Engineering Science, October 12-14, Northwestern University, Evanston, IL, October 13, 2011.

15. J. T. Fong, "A Bayesian Approach to Estimating the Uncertainty of Fatigue Life of Pressure Vessels and Piping," $39^{\text {th }}$ Review of Progress in QNDE Meeting, July 15-20, 2012, Denver, CO, July 18, 2012.

16. J. T. Fong, "QNDE: Key to Developing CuttingEdge Tools for Manufacturing and Product Longevity," $39^{\text {th }}$ Review of Progress in QNDE Meeting, July 15-20, 2012, Denver, CO, July 19, 2012.

17. A. Gueye, "A Game Theoretic Framework for Network Vulnerability Assessment and Mitigation," GameSec2012, Budapest, Hungary, November 6, 2012.

18. A. Gueye, "Toward a Metric for Communication Network Vulnerability to Attacks: A Game Theoretic Approach," GameNets2012, Vancouver, BC, Canada, May 25, 2012.

19. A. Kearsley, "On Calculating Distances Between Chemical Spectra," 8th International Conference on Information, Communications \& Signal Processing (ICICS), Singapore, December 15, 2011.

20. A. Kearsley, "Calibrating Chemical Spectrometers," American Society for Mass Spectrometry, Vancouver, Canada, June 21, 2012.

21. A. Kearsley, "Wassterstein Metric and Multidimensional Scaling for Compound Searching," University of Calgary, July 20, 2012.

22. P. S. Kuo, J. S. Pelc, O. Slattery, M. M. Fejer and $\mathrm{X}$. Tang, "Photon Temporal Correlations Measured Using a Dual-Channel Upconversion Detector," Frontiers in Optics Conference, Rochester, NY, October 14, 2012.

23. S. Jordan, "Super-polynomial Quantum Speedups," Workshop on Post-Quantum Cryptog- raphy and Quantum Algorithms, Lorentz Center, Leiden, Netherlands. November 5, 2012.

24. Y.-K. Liu, "Universal Low-rank Matrix Recovery from Pauli Measurements," Workshop on Quantum Information: Codes, Geometry and Random Structures, Centre de Recherches Mathématiques, Montreal, Canada, October 24, 2011.

25. Y.-K. Liu, "Sub-tomography and Fidelity Estimation," Workshop on Quantum Tomography, Centre for Quantum Technologies, Singapore, November 30, 2011.

26. Y.-K. Liu, "Universal Low-rank Matrix Recovery from Pauli Measurements," Neural Information Processing Systems (NIPS) Conference, Granada, Spain, December 14, 2011.

27. Y.-K. Liu, "How to Interpret a Large Density Matrix," Workshop on Quantum Computation, Verification and Validation (QCVV), Bethesda, MD, May 1, 2012.

28. H. Mahboubi, W. Masoudimansour, A, Aghdam, K. Sayrafian, V. Marbukh, "Maximum Life Span Strategy for Target Tracking in Mobile Sensor Networks," IEEE American Control Conference (ACC), June 27-29, 2012.

29. V. Marbukh, "Towards Modeling Effect of Agents' Bounded Rationality on Performance of Selfish Resource Allocation," the Latsis Symposium on Econophysics, ETH-Zurich, Switzerland, 2012.

30. V. Marbukh, "Risks/benefits of Selfish Network Operations \& Management in Dynamic Environment," 13th IEEE/IFIP Network Operations and Management Symposium (NOMS 2012), Maui, HI, 2012.

31. V. Marbukh, "Towards Modeling Effect of Packetlevel TCP Dynamics on Internet Operations and Management," 13th IEEE/IFIP Network Operations and Management Symposium (NOMS 2012), Maui, HI, 2012.

32. N. S. Martys, C. F. Ferraris, W. L. George, S. G. Satterfield and M. T. Olano, "Computational Based Study of Suspension Flow in a Vane Rheometer," 83rd Annual Meeting of the Society of Rheology, Cleveland, Ohio, October 9-13, 2011.

33. W. F. Mitchell, "Experience With MPI and OpenMP in an Adaptive FEM Code," SIAM Conference on Parallel Processing for Scientific Computing, Savannah, GA, February 15, 2012.

34. A. P. Peskin, A. Dima, G. Saiprasad, "An Automated Method for Locating Phantom Nodules in Anthropomorphic Thoracic Phantom CT Studies," 
International Conference on Image Processing and Computer Vision, Las Vegas, NV, July 16-19, 2012.

35. R. Pozo, "Efficient Q-matrix Computation for the Visualization of Complex Networks," Workshop on Complex networks and Their Applications, The $8^{\text {th }}$ International Conference on Signal Image Technology \& Internet Based Systems, Naples, Italy, November 25-29, 2012.

36. S. Ressler, "Not Your Mother's Web3D: Integrating X3DOM, jQuery and HTML5," Web3D 2012, Los Angeles, CA, August 4, 2012.

37. S. Ressler, "Web-based Declarative 3D Graphics for Anthropometry Visualization and Education," The Graphical Web 2012, Zurich, Switzerland, September 13, 2012.

38. B. Saunders, B. Antonishek and Q. Wang, "Adaptive Composite B-Spline Grid Generation for Interactive 3D Visualizations," Eighth International Conference on Mathematical Methods for Curves and Surfaces MASCOT/ISGG 2012 (IMACS Workshop and International Society for Grid Generation Conference), Las Palmas de Gran Canaria, Spain, October 24, 2012.

39. B. Saunders, "Adaptive Composite B-Spline Mesh Generation for the Visualization of Complex Function Surfaces," Eighth International Conference on Mathematical Methods for Curves and Surfaces, Oslo, Norway, June 29, 2012.

40. B. Saunders, "Adaptive Grid Generation Using the Composition of Tensor Product B-Splines," SIAM Conference on Geometric and Physical Modeling, Orlando, Florida, October 27, 2011.

41. J. Shook, "Affiliation Networks," Mathematics Department, University of Mississippi, Oxford, MS, October 6, 2011.

42. J. Shook, "Greater than 1-tough k-trees without a 2-factor," International Conference on Cycles in Graphs, Nashville, TN, June 1, 2012.

43. V. Taroudaki and D. P. O'Leary, "Reconstruction of Images: Computation of Confidence Intervals," SIAM Conference on Imaging Science, Philadelphia, May 2012.

44. V. Taroudaki and D. P. O'Leary, "Confidence Intervals of an Image Reconstruction," Modern Mathematical Methods in Science and Technology (M3ST) Conference, Kalamata, Greece, August 2012.

45. J. Terrill and S. Belloge, "Visualization and Analysis of the National Vulnerability Database," NIST
Booth Demo, 8th Annual IT Security Automation Conference, Baltimore MD, October 3-5, 2012.

46. L. Valentini, G. Artioli, J.W. Bullard, S.G. Satterfield, "Modeling the Effect of Calcium Sulfate on C3S Hydration: Implications for the Origin of the Optimum Sulfate," 3rd Advances in CementBased Materials: Characterization, Processing, Modeling and Sensing, Austin, Texas, June 10-12, 2012.

47. W. Yang, K. Sayrafian, "Radiation Pattern of an UWB Wearable Antenna: A Preliminary Study," $7^{\text {th }}$ International Conference on body Area Networks (BodyNets), September 24-26, 2012.

48. W. Yang, K. Sayrafian, "Interference Mitigation for Body Area Networks," $22^{\text {nd }}$ Annual IEEE International Symposium on Personal, Indoor and Mobile Radio Communications (PIMRC), September 11-14, 2011.

\section{Web Services}

1. Digital Library of Mathematical Functions ${ }^{27}$ : a repository of information on the special functions of applied mathematics.

2. Guide to Available Mathematical Functions ${ }^{28}$ : a virtual repository of mathematical software components.

3. Matrix Market ${ }^{29}$ : a repository of matrices for testing of algorithms and software for numerical linear algebra.

4. SciMark ${ }^{30}$ : a benchmark for scientific computing in Java.

\section{$\underline{\text { Software Released }}{ }^{31}$}

1. ACPT: Access Control Policy Test - V. Hu, D. R. Kuhn and R. Kacker

2. ACTS: Combinatorial Test Suite Generation with Support of Constraints. Version 2.7 - Y. Lei, D. R. Kuhn and R. Kacker

3. Combinatorial Coverage Measurement. Version 1.0 - D. R. Kuhn and R. Kacker

\footnotetext{
27 http://dlmf.nist.gov/

28 http://gams.nist.gov/

29 http://math.nist.gov/matrixmarket/

30 http://math.nist.gov/scimark/

31 In cases where multiple versions have been released during the year we only list the last.
} 
4. C-PUT: Tool for Prioritization of User-Session Based Test Suites - R. Bryce, S. Sampath, D. R. Kuhn and R. Kacker

5. Graphicality Testing Routines. Released to NetworkX - B. Cloteaux

6. NGraph++ Toolkit: Network Graph Toolkit for the analysis and simulation of large scale networks, Version 4.5 - R. Pozo

7. OOMMF: Object Oriented Micromagnetic Framework. Version 1.2a5 - D. Porter and M. Donahue

8. PHAML: Parallel Hierarchical Adaptive MultiLevel finite element program for the solution of elliptic partial differential equations, Versions 1.10.0, 1.11.0 - W. Mitchell.

9. Tcl/Tk. Versions 8.5.11, 8.5.12, 8.6b3 - D. Porter

10. trofs: Read-only virtual filesystem support for Tcl. Version 0.4.6 - D. Porter

11. varpro. $\mathrm{m}^{32}:$ Variable projection Matlab software for least squares problems - D. P. O'Leary and B. W. Rust

\section{Conferences, Minisymposia, Lecture Series, Courses \\ MCSD Seminar Series}

B. Cloteaux served as Chair of the ACMD Seminar Series. There were 29 talks presented during this period.

1. J. Shook (ACMD), "On Finding A Minimum Toughness Condition for a k-tree to be Hamiltonian," November 29, 2011.

2. C. Hogg (Ceramics Division, NIST), "What do Noisy Datapoints Tell Us About the True Signal?" January 17, 2012.

3. A. Carasso (ACMD), "Some New Results in Inverse Reconstruction,” January 31, 2012.

4. S. Jordan (ACMD), "Quantum Algorithms for Quantum Field Theories,” February 21, 2012.

5. B. Roy (Indian Statistical Institute), "Application of PBIBD in Cryptology and Security," March 6, 2012.

6. W. Mitchell (ACMD), "Comparison of hpAdaptive Finite Element Strategies," March 13, 2012.

\footnotetext{
${ }^{32}$ http://www.cs.umd.edu/ oleary/software/varpro.m
}

7. R. Goldin (George Mason University), "Exploration of a Random Dot Product Graph Model for Neuronal Connectivity," March 20, 2012.

8. E. Moseman (ACMD), "Network Reliability: Approximation Algorithms," March 30, 2012.

9. T. Taylor (National Cancer Institute), "Finding and Quantifying Protein Monomeric Structural Pseudo-Symmetry," April 24, 2012.

10. R. Song (University of Illinois), "Subordinate Brownian Motions and Their Applications," May 2, 2012.

11. Y. Hu (AT\&T Labs), "Visualizing Data with Graphs and Maps," May 7, 2012.

12. Y. Liu (ACMD), "Universal Low-Rank Matrix Recovery from Pauli Measurements," May 15, 2012.

13. S. Casey (American University), "Signal Adaptive Frame Theory," May 22, 2012.

14. M. Kohlhase (Jacobs University), "The Planetary System: Active Documents and a Web3.0 for Math," May 30, 2012.

15. D. LaMar (The College of William and Mary), "Split Digraphs and Their Applications," June 19, 2012.

16. J. Walrand (University of California at Berkeley), “A Benes Packet Network," June 22, 2012.

17. Z. Di (George Mason University), "Applications and Recent Developments of Multilevel Optimization Framework (MG/OPT)," July 24, 2012.

18. T. Massard (CEA, France), "Numerical Experiments and Data Mining in Huge Experimental Databases at Selected Nuclear Energy and Alternative Energy Research Centers in France," July 26, 2012.

19. K. Petersen (New York University), "Finding Minimal Energy Paths for Droplets on Superhydrophobic Surfaces: A Phase Field Approach," July 30, 2012.

20. R. La (University of Maryland), "Secondary Spectrum Trading Market - Auction-based Approach to Spectrum Allocation and Profit Sharing," August 14, 2012.

21. C. Dunne (University of Maryland), "Measuring and Improving the Readability of Network Visualizations," August 28, 2012.

22. D. O'Leary (University of Maryland), "Image Restoration Using Machine Learning," September 11, 2012. 
23. J. De Loera (University of California at Irvine), "Integrals of Polynomials over Convex Polytopes: Combinatorics and Algorithms," September 19, 2012.

24. Walter Morris (George Mason University), "Clique Number and Chromatic Number of Graphs Defined by Convex Geometries," October 18, 2012.

25. Jaideep Ray (Sandia National Laboratories, Livermore), "Generating Conditional Realizations of Graphs and Fields Using Markov Chain Monte Carlo," November 5, 2012.

26. Kyle Hickmann (Tulane University), "Inverse Problems for Material Characterization Using Thermoacoustic Imaging,” November 16, 2012.

27. Zydrunas Gimbutas (Courant Institute, New York University), "Robust High Order Integral Equation Solvers for Electromagnetic Scattering in Complex Geometries," November 29, 2012.

28. Alfred Carasso (ACMD), "Hazardous Continuation Backward in Time in Nonlinear Parabolic Equations and an Experiment in Deblurring Nonlinearly Blurred Imagery," December 4, 2012.

29. Amanda P. Streib (ACMD), "Mixing Times of Self-Organizing Lists and Biased Permutations," December 12, 2012.

\section{Conference Committees}

1. Wesley Griffin, Publicity Chair, ACM SIGGRAPH Symposium on Interactive 3D Graphics and Games, 2013.

2. A. Gueye, Technical Program Chair, $3^{\text {rd }}$ Conference on Decision and Game Theory for Security, (GameSec2012), 2012.

3. A. Gueye, Member, Organizing Committee, $2^{\text {nd }}$ Conference on Decision and Game Theory for Security (GameSec2011), 2011.

4. F. Hunt, Co-Chair, Steering Committee, Infinite Possibilities Conference, University of Maryland, Baltimore County, March 30-31' 2012.

5. R. Kacker, Co-Chair, First International Workshop on Combinatorial Testing (IWCT) in conjunction with the 5th IEEE International Conference on Software Testing, Verification and Validation (ICST), Montreal Canada, April 17-21, 2012.

6. S. Jordan, Member, Program Committee, Quantum Information Processing, (QIP) 2013.
7. A. Kearsley, Member, Organizing Committee, 2nd IEEE Conference on Control, Systems \& Industrial Informatics, 2012.

8. V. Marbukh, Co-Organizer, Second NIST - Alcatel/Lucent Bell Labs Workshops on Large-Scale Geometry of Complex Networks, NIST, Gaithersburg, MD, March 14, 2012.

9. W. F. Mitchell, Member, Scientific Committee, International Conference of Numerical Analysis and Applied Mathematics (ICNAAM 2012), Kos, Greece, September 19-25, 2012.

10. D. P. O'Leary, Member, Program Committee, NAACL-HLT 2012 Workshop on Evaluation Metrics and System Comparison for Automatic Summarization, Montreal, Quebec, Canada, June 8, 2012.

11. D. P. O'Leary, Member, Organizing Committee, SIAM Conference on Applied Linear Algebra (LA12), Valencia, Spain, June 18-22, 2012.

12. Sandy Ressler, Tutorial Chair, 18th International Conference on 3D Web Technology, 2013.

13. Sandy Ressler, Member, Program Committee, WWW2012 Workshop on Declarative 3D for the Web Architecture, 2012.

14. K. Sayrafian, Member, Organizing Committee and Track Chair, $22^{\text {nd }}$ Annual IEEE International Symposium on Personal, Indoor and Mobile Radio Communications (PIMRC), Sept. 11-14, 2011.

15. K. Sayrafian, Member, Organizing Committee and Track Chair, $7^{\text {th }}$ International Conference on Body Area Networks (BodyNets), Sept. 24-26, 2012.

16. K. Sayrafian, Member, Steering Committee, NSF Planning Workshop on Medical Device Innovation Using Cyber Physical Systems, July 18-19, 2012.

17. K. Sayrafian, Member, Technical Program Committee, 6th International Symposium on Medical Information and Communication Technology (ISMICT), March 26-29, 2012.

18. K. Sayrafian, Member, Technical Program Committee and International Advisory Board, 4th International Symposium on Applied Sciences in Biomedical and Communication Technologies (ISABEL), October 26-29, 2011. 


\section{Other}

1. J. T. Fong, Instructor, Modern Statistics, Data Analysis and Specimen/Structural Reliability Modeling, One-day Shortcourse, American Ceramic Society Fall Conference, Columbus, OH, October 16, 2011.

2. F. Hunt, Instructor, Stochastic Processes, Howard University, Fall 2012.

3. R. Kacker and Y. Lei, Presenters, Workshop on Combinatorial Testing for Software and Systems in conjunction with the International Conference on Quality and Reliability Engineering (ICQRE2011) organized by the Indian Statistical Institute, Bangalore, India, December 22, 2011.

4. A. J. Kearsley, Mentor, Mathematical Modeling in Industry XVI -- A Workshop for Graduate Students, University of Calgary, Canada, June 18-27, 2012.

5. Y. Parker and J. Terrill, Co-Organizers, NIST booth, SC11, the 2011 International Conference for High Performance Computing, Networking, Storage and Analysis, Seattle, WA, November 1319 ,

6. Y. Parker and J. Terrill, Co-Organizers, NIST booth, SC12, the 2012 International Conference for High Performance Computing, Networking, Storage and Analysis, Salt Lake City, Utah, November 13-19, 2012.

\section{Other Professional Activities}

\section{Internal}

1. I. Beichl, ITL Director, Summer Undergraduate Research Fellowship (SURF) Program.

2. R. Boisvert, NIST Scientific Computing Steering Group.

3. R. Boisvert, ITL Diversity Committee.

4. S. Glancy, NIST Boulder SURF Committee.

5. S. Glancy, President, Boulder Commerce Children's Center Association.

6. W. Mitchell, ITL Awards Committee.

7. A. O'Gallagher, ITL Diversity Committee.

8. S. Ressler, NIST Lobby Display Advisory Council.

\section{External}

\section{Editorial}

1. I. Beichl, Editor-in-Chief, Computing in Science \& Engineering.

2. R. Boisvert, Associate Editor, ACM Transactions on Mathematical Software.

3. R. Boisvert, Editor, Numerical Analysis, Mathematical Software and Computational Engineering, Finance and Science areas, Computing Research Repository (CoRR) preprint service. ${ }^{33}$

4. A. Dienstfrey, Associate Editor, International Journal of Uncertainty Quantification.

5. A. Kearsley, Member, Editorial Board, ISRN Journal on Applied Mathematics.

6. D. Lozier, Associate Editor, Journal of Numerical Analysis, Industrial and Applied Mathematics.

7. W. Mitchell, Associate Editor, Journal of Numerical Analysis, Industrial and Applied Mathematics.

8. D. P. O'Leary, Editor-in-Chief, SIAM Journal on Matrix Analysis and Applications.

9. D. P. O'Leary, Member, Editorial Board, Education Section, SIAM Review.

10. D. P. O'Leary, Member, Editorial Board, SIAM Books.

11. D. P. O'Leary, Department Editor, Your Homework Assignment, Computing in Science \& Engineering.

12. F. Potra, Regional Editor for the Americas, Optimization Methods and Software.

13. F. Potra, Associate Editor, Journal of Optimization Theory and Applications.

14. F. Potra, Associate Editor, Numerical Functional Analysis and Optimization.

15. F. Potra, Associate Editor, Optimization and Engineering.

16. R. Pozo, Associate Editor, ACM Transactions on Mathematical Software.

17. K. Sayrafian, Guest Editor, International Journal on Wireless Information Networks (IJWIN), Special Issue on Wireless Technologies in Healthcare, Volume 19, Issue 3, 2012.

18. K. Sayrafian, Guest Editor, International Journal of Ultra Wideband Communications and Systems, Special Issue on Applications of Ultra Wideband

33 http://www.arXiv.org/ 
Technology in Healthcare, Volume 2, Issue 2, 2011.

\section{Boards and Committees}

1. I. Beichl, Member, Magazine Operations Committee, IEEE Computer Society.

2. R. Bohn, Member, Cloud Computing Scoping Subcommittee, President's National Security Telecommunications Advisory Committee (NSTAC).

3. R. Bohn. Co-chair, Faster Administration of Science and Technology Education and Research (FASTER) Community of Practice, Networking and Information Technology R\&D (NITRD) Program.

4. R. Boisvert, Chair, International Federation for Information Processing's (IFIP) Working Group 2.5 (Numerical Software).

5. R. Boisvert, Co-chair, Publication Board, Association for Computing Machinery (ACM).

6. R. Boisvert, Member, Program Review Committee, Center for Computing Sciences, Institute for Defense Analysis.

7. R. Boisvert, Member, External Review Committee, Computer Science Department, George Washington University.

8. R. Boisvert, Member, Subcommittee on the Materials Genome Initiative, Committee on Technology, National Science and Technology Council.

9. A. Dienstfrey, Member, International Federation for Information Processing's (IFIP) Working Group 2.5 (Numerical Software).

10. F. Hunt, Member, Advisory Board, National Institute of Mathematical Biological Synthesis, University of Tennessee, Knoxville, Tennessee.

11. E. Knill, Member, Technical Advisory Panel, Quantum Computer Science Program, IARPA.

12. D. Lozier, Member, Steering Committee, Painleve Project.

13. D. P. O'Leary, Member, Oversight Committee, Gene Golub SIAM Summer Schools.

14. D. P. O'Leary, Member, Advisory Committee, SIAM Linear Algebra Activity Group International Summer School on Numerical Linear Algebra.

15. V. Marbukh, Member, Network Complexity Research Group, Internet Research Task Force (IRTF).
16. G. B. McFadden, Member, Selection Committee, SIAM Kleinman Prize.

17. B. Miller, Member, Math Working Group, World Wide Web Consortium.

18. D. Porter, Member, Release Manager, Tcl Core Team.

19. S. Ressler, Member, Declarative 3D for the Web Architecture Community Group ${ }^{34}$.

20. B. Saunders, Member, MAA Committee on Business, Industry and Government Mathematics.

21. B. Saunders, Member, Advisory Group for NSF CoSMIC Scholars Program, Towson University.

22. B. Saunders, Webmaster, SIAM Activity Group on Orthogonal Polynomials and Special Functions.

23. B. Saunders, OP-SF Talk listserv Moderator, SIAM Activity Group on Orthogonal Polynomials and Special Functions.

24. K. Sayrafian, Member, IEEE 802.15 Working Group on Wireless Personal Area Networks.

25. K. Sayrafian, Member, COST-IC1004, Action on Cooperative Radio Communications for Green Smart Environments, Body Area Networking Group.

26. K. Sayrafian, Member, Wireless Medical Technologies Working Group, National Institute for Biomedical Imaging and Bioengineering (NIBIB/NIH).

27. K. Sayrafian, Member, Thesis Defense Committee, Worcester Polytechnic Institute (Pranay Swar, Ph.D., May $30^{\text {th }}$, 2012, Yunxing Ye, M.Sc., July 31, 2012)

28. J. Terrill, Member, High End Computing Research and Development and Infrastructure Interagency Working Groups, Networking and Information Technology R\&D (NITRD) Program.

\section{Grants Awarded}

ACMD awards a small amount of funding through the NIST Measurement Science Grants Program for projects that make direct contributions to its research programs. Often such grants support direct cooperation between an external awardee and ACMD. This year the following research grants were awarded.

1. Concordia University: Energy Efficient Robust Control of Mobile Sensors in Environments with

34 http://www.w3.org/community/declarative3d/ 
Obstacles, \$99,000 (3 years). PI: Dr. Amir Aghdam.

2. National Physical Sciences Consortium: NIST-ITL, National Physical Science Consortium Project, \$297,000 (3 years). PI: Dr. James Powell.

3. Santa Fe Institute: Innovative Workshop Program in Network Science, Biologically Inspired Computation, and Computer Security, \$300,000 (5 years). PIs: Dr. Chris Wood and Drs. Chris Wood and Cris Moore.

4. University of California at San Diego: Micromagnetic Finite Difference Simulators on GPUs, \$180,000 (3 years). PI: Vitaliy Lomakin.

5. University of Edinburgh: Special Functions: Next Steps for the DLMF, \$173,513 (3 years). PI: Dr. Adri Olde Daalhuis.

6. University of Minnesota: Uncertainty Quantification for Molecular Dynamics Simulations for Bulk Behavior of Advanced Composites, \$283,387 (3 years). PI: Dr. Fadil Santosa.

7. University of Texas at Arlington: Combinatorial Testing for Complex Systems, \$330,384 (3 years). PI: Dr. Yu Lei.

\section{External Contacts}

ACMD staff members make contact with a wide variety of organizations in the course of their work. Examples of these follow.

\section{Industrial Labs}

Alcatel Lucent Bell Laboratories

ASSET InterTech

CLO Virtual Fashion, Inc.

Foray Technologies

IBM India, Bangalore, India

Infosys Technologies, Bangalore, India

Mentor Graphics

Microsoft Research New England

MPACT Corporation

Northrup Grumman

NVIDIA

Orbital Sciences Corporation

OSI Systems

Palantir Technologies

Philips Research Asia Bangalore, India

Schwarz Forensic Enterprises

Walt Disney Animation Studios

Watson Adventures

\section{Government/Non-profit Organizations}

Air Force Office of Scientific Research
Argonne National Laboratory

Army Research Laboratory

American Society for Mechanical Engineering

Association for Computing Machinery

Basque Center for Applied Mathematics (Spain)

CEA (France)

CENAM (National Metrology Institute of Mexico)

Center for Integration of Medicine and Innovative

Technology

China Special Equip. Inspection and Res. Inst. (China)

CNRS/ESPCI, France

Department of Energy

Electric Power Research Institute

Federal Communications Comission

Food and Drug Administration

IARPA

IDA Center for Computing Sciences

IEEE

Indian Statistical Institute (India)

International Federation for Information Processing

Internet Research Task Force

Lawrence Livermore National Laboratory

Lieber Institute

NASA Ames Research Center

NASA Indep. Verification and Validation Facility

Nat. Inst. for Biomedical Imaging and Bioengineering

National Institutes of Health

National Institute of Information and Communication Technology (Japan)

NOAA

National Physical Laboratory (UK)

National Physical Sciences Consortium

National Renewable Energy Laboratory

National Science Foundation

Nuclear Regulatory Commission

Oak Ridge National Laboratory

Pacific Northwest National Laboratory

Perimeter Institute (Canada)

Physikalisch-Technische Bundesanstalt (Germany)

Sandia National Laboratories

Santa Fe Institute

Savanah River National Laboratory

Society for Industrial and Applied Mathematics

Southwest Research Institute

Swedish Defense Research Agency (Sweden)

Theiss Research

West Health Institute

World Wide Web Consortium

\section{Universities}

Adam Mickiewicz University (Poland)

AGH University of Science and Technology (Poland)

Beijing Institute of Technology (China)

Boston University

Brown University

Budapest University of Technology and Economics (Hungary) 
California Institute of Technology

Carnegie Mellon University

China University of Petroleum (China)

Chinese Culture University (China)

City University of Hong Kong (China)

College of William and Mary

Concordia University (Canada)

Cornell University

Courant Institue of Mathematical Sciences

Dalian University of Technology (China)

East China University of Sci. and Technology (China)

Ecole Polytechnique de Montreal (Canada)

ETH, Zurich (Switzerland)

Federal University of Rio Grande do Norte (Brazil)

Freiburg Institute for Advanced Studies (Germany)

Freie Universität Berlin

George Mason University

George Washington University

Georgia Institute of Technology

Georgia State University

Gothenburg University (Sweden)

Hanoi Institute of Mathematics (Vietnam)

Howard University

IGBMC, Strasbourg (France)

Illinois Institute of Technology

Imperial College, London (UK)

Indiana University

Institut Supérieur d'Informatique de Modélisation et de leurs Applications (ISIMA) (France)

Institute for Computational and Experimental Math.

Institute for Mathematics and Its Applications

Iowa State University

Istituto per le Applicazioni del Calcolo (Italy)

Jacobs University Bremen (Germany)

Johns Hopkins University

Kansas State University

Karlsruhe Institute of Technology (Germany)

Louisiana State University

Macquarie University (Australia)

Montana State University

Morgan State University

Nanyang University (Singapore)

National University of Singapore (Singapore)

New York University

Northern Illinois University

Ningbo University, Ningbo (China)

Northwestern University

Old Dominion University

Open University (UK)

Oregon State University

Oslo University (Norway)

Purdue University

Shandong University (China)

Stanford University

Technical University of Berlin (Germany)
Tel Aviv University (Israel)

The College of William and Mary

Tokyo Institute of Technology (Japan)

Towson University

Tsinghua University (China)

Tulane University

UCLA

University of Alabama at Birmingham

University of Antwerp (Belgium)

University of Bologna (Italy)

University of Colorado

University of British Columbia

University of California at Berkeley

University of California at Davis

University of California at Irvine

University of California at San Diego

University of California at Santa Cruz

University of Campinas (Brazil)

University of Cantabria (Spain)

University of Central Florida

University of Colorado at Denver

University of Edinburgh (UK)

University of Erlangen (Germany)

University of Florida

University of Freiburg

University of Hong Kong (China)

University of Illinois

University of Indiana

University of Maryland

University of Maryland Baltimore County

University of Metz (France)

University of Miami

University of Minnesota

University of Mississippi

University of New Mexico

University of Oulu (Finland)

University of Pennsylvania

University of Pittsburgh

University of Rochester

University of South Carolina

University of Sydney (Australia)

University of Tennessee

University of Texas at Arlington

University of Utah

University of Waikato (New Zealand)

University of Washington

University of Waterloo (Canada)

University of Wisconsin

University of West Bohemia (Czech Republic)

University of Zagreb (Croatia)

Virginia Tech

Worcester Polytechnic Institute

Wroclaw University (Poland)

Yokohama National University (Japan) 


\section{Part V}

Appendix 


\section{$\underline{\text { Staff }}$}

ACMD consists of full time permanent staff located at NIST laboratories in Gaithersburg, MD and Boulder, CO. This is supplemented with a variety of special appointments. The following list reflects all appointments held during any portion of the reporting period (October 2011 - December 2012). For students and interns, see Table 2, page 11. (*) Denotes staff at NIST Boulder.

\section{Division Staff}

Ronald Boisvert, Chief, Ph.D. (Computer Science), Purdue University, 1979

Robin Bickel, Secretary

Catherine Graham, Secretary

Robert Bohn, Ph.D. (Physical Chemistry), University of Virginia, 1991

Alfred Carasso, Ph.D. (Mathematics), University of Wisconsin, 1968

Roldan Pozo, Ph.D. (Computer Science), University of Colorado at Boulder, 1991

Christopher Schanzle, B.S. (Computer Science), University of Maryland Baltimore County, 1989

\section{Mathematical Analysis and Modeling Group}

Timothy Burns, Leader, Ph.D. (Mathematics), University of New Mexico, 1977

*Bradley Alpert, Ph.D. (Computer Science), Yale University, 1990

*Andrew Dienstfrey, Ph.D. (Mathematics), New York University, 1998

Jeffrey Fong, Ph. D. (Applied Mechanics and Mathematics), Stanford University, 1966

David Gilsinn, Ph.D. (Mathematics), Georgetown University, 1969

Fern Hunt, Ph.D. (Mathematics), New York University, 1978

Raghu Kacker, Ph.D. (Statistics), Iowa State University, 1979

Anthony Kearsley, Ph.D. (Computational and Applied Mathematics), Rice University, 1996

Peter Ketcham. M.S. (Mathematics), University of Minnesota, 1997

Geoffrey McFadden, NIST Fellow, Ph.D. (Mathematics), New York University, 1979

*Agnes O'Gallagher, M.S. (Applied Mathematics), University of Colorado at Boulder, 1991

Bert Rust, Ph.D. (Astronomy), University of Illinois at Urbana-Champaign, 1974

NIST-ARRA Postdoctoral Fellows

Asha Nurse, Ph.D. (Mechanical Engineering), Brown University, 2011

Ismet Sahin, Ph.D. (Electrical and Computer Engineering), University of Pittsburgh, 2006

Faculty Appointee (Name, Degree / Home Institution)

Daniel Anderson, Ph.D. / George Mason University

Saul Gass, Ph.D. / University of Maryland College Park

Dianne O’Leary, Ph.D. / University of Maryland College Park

Florian Potra, Ph.D. / University of Maryland Baltimore County

Guest Researchers (Name, Degree / Home Institution)

Mirit Aladjem, Ph.D. / National Institutes of Health

James Benson, Ph.D. / Northern Illinois University

David Cotrell, Ph.D. / CD-Adapco

*John Gary, Ph.D. / NIST (retired)

*Daniel Kaslovsky, Ph.D. / University of Colorado

$\mathrm{Yu}$ (Jeff) Lei, Ph.D. / University of Texas at Arlington

P. Aaron Lott, Ph.D. / Lawrence Livermore National Laboratory

Itzel Dominquez Mendoza / Centro Nacional de Metrología, Mexico 
Bruce Murray, Ph.D. / SUNY Binghamton

Santanu Sarkar, Ph.D. / Indian Statistical Institute

Christoph Witzgall, Ph.D., NIST Scientist Emeritus

\section{Mathematical Software Group}

Daniel Lozier, Leader, Ph.D. (Applied Mathematics), University of Maryland, 1979

Michael Donahue, Acting Leader Ph.D. (Mathematics), Ohio State University, 1991

Javier Bernal, Ph.D. (Mathematics), Catholic University, 1980

Stephen Langer, Ph.D. (Physics), Cornell University, 1989

Marjorie McClain, M.S. (Mathematics), University of Maryland College Park, 1984

Bruce Miller, Ph.D. (Physics), University of Texas at Austin, 1983

William Mitchell, Ph.D. (Computer Science), University of Illinois at Urbana-Champaign, 1988

Donald Porter, Ph.D. (Electrical Engineering), Washington University, 1996

Bonita Saunders, Ph.D. (Mathematics), Old Dominion University, 1985

NRC Postdoctoral Associates

Howard Cohl, Ph.D. (Mathematics), University of Auckland, 2010

Faculty Appointees (Name, Degree / Home Institution)

Frank Olver, D.Sc. / University of Maryland College Park

G.W. Stewart, Ph.D. / University of Maryland College Park

Abdou Youssef, Ph.D. / George Washington University

Guest Researchers (Name, Degree / Home Institution)

Gunay Dogan, Ph.D. / Theiss Research

Adri Olde Daalhuis, Ph.D. / University of Edinburgh

Qiming Wang / NIST (retired)

\section{Computing and Communications Theory Group}

Ronald Boisvert, Acting Leader

Isabel Beichl, Project Leader, Ph.D. (Mathematics), Cornell University, 1981

Brian Cloteaux, Ph.D. (Computer Science), New Mexico State University, 2007

*Scott Glancy, Ph.D. (Physics), University of Notre Dame, 2003

Barry Hershman, A.A. (Electronics Engineering), Capitol College, 1979

Stephen Jordan, Ph.D. (Physics), Massachussetts Institute of Technology, 2008

*Emanuel Knill, NIST Fellow, Ph.D. (Math), University of Colorado at Boulder, 1991

Yi-Kai Liu, Ph.D. (Computer Science), University of California, San Diego, 2007

Vladimir Marbukh, Ph.D. (Mathematics) Leningrad Polytechnic University, 1986

Oliver Slattery, M.S. (Electrical Engineering), Johns Hopkins University, 2008

Kamran Sayrafian-Pour, Ph.D. (Electrical and Computer Engineering), University of Maryland, 1999

Xiao Tang, Project Leader, Ph.D (Physics), Chinese Academy of Sciences, 1985

NRC Postdoctoral Associates

Elizabeth Moseman, Ph.D. (Mathematics), Dartmouth College, 2007

James Shook, Ph.D. (Mathematics), University of Mississippi, 2010

Amanda Streib, Ph.D. (Mathematics), Georgia Institute of Technology, 2012

Noah Streib, Ph.D. (Mathematics), Georgia Institute of Technology, 2012

NIST-ARRA Postdoctoral Fellows

Assane Gueye, Ph.D. (Computer Science), University of California, Berkeley, 2011 


\section{Contractors}

Lijun Ma, Ph.D. (Optical Engineering), Tsinghua University, 2001

Alan Mink, Ph.D. (Electrical Engineering), University of Maryland, 1980

Faculty Appointees (Name, Degree / Home Institution)

James Lawrence, Ph.D. / George Mason University

Guest Researchers (Name, Degree / Home Institution)

*Bryan Eastin, PhD. / Northrup Grumman

Yong-Su Kim, Ph.D. / Pohang University of Science and Technology

Paulina Kuo, Ph.D. / Joint Quantum Institute, University of Maryland

Francis Sullivan, Ph.D. / IDA Center for Computing Sciences

\section{High Performance Computing and Visualization Group}

Judith Terrill, Leader, Ph.D. (Information Technology), George Mason University, 1998

Yolanda Parker, Office Manager

William George, Ph.D. (Computer/Computational Science), Clemson University, 1995

Terence Griffin, B.S. (Mathematics), St. Mary's College of Maryland, 1987

Wesley Griffin, M.S. (Computer Science), University of Maryland Baltimore County, 2010

John Hagedorn, M.S. (Mathematics), Rutgers University, 1980

John Kelso, M.S. (Computer Science), George Washington University, 1984

*Adele Peskin, Ph.D. (Chemical Engineering), University of Colorado at Boulder, 1985

Sandy Ressler, M.F.A. (Visual Arts), Rutgers University, 1980

Steven Satterfield, M.S. (Computer Science), North Carolina State University, 1975

James Sims, Ph.D. (Chemical Physics), Indiana University, 1969

Faculty Appointees (Name, Degree / Home Institution)

Marc Olano, Ph.D. / University of Maryland Baltimore County 


\section{Glossary of Acronyms}

\begin{tabular}{|c|c|}
\hline $2 \mathrm{D}$ & two dimensions \\
\hline 3D & three dimensions \\
\hline A\&S & Abramowitz \& Stegun (NBS Handbook of Mathematical Functions, 1964) \\
\hline $\mathrm{ACM}$ & Association for Computing Machinery \\
\hline ACMD & NIST/ITL Applied and Computational Mathematics Division \\
\hline ACTS & Advanced Combinatorial Testing System \\
\hline AIP & American Institute of Physics \\
\hline AISI & American Iron and Steele Institute \\
\hline ALCF & Argonne Leadership Computing Facility \\
\hline AMS & American Mathematical Society \\
\hline ANSI & American National Standards Institute \\
\hline ANTD & ITL Advanced Networking Technology Division \\
\hline APD & avalanche photo diode (photon detector) \\
\hline ARRA & American Recovery and Reinvestment Act \\
\hline $\operatorname{arXiv}$ & preprint archive housed at Cornell University (http://arxiv.org/) \\
\hline ASCE & American Society of Civil Engineers \\
\hline ASME & American Society of Mechanical Engineers \\
\hline ASTM & ASTM International, formerly known as the American Society for Testing and Material \\
\hline BAN & body area network \\
\hline Caltech & Californial Institute of Technology \\
\hline CENAM & Centro Nacional de Metrología (Mexico) \\
\hline $\mathrm{CI}$ & configuration interaction \\
\hline CMOS & complementary metal-oxide semiconductor \\
\hline CNRS & Centre National de la Recherche Scientifique (France) \\
\hline CNST & NIST Center for Nanoscale Science and Technology \\
\hline CODATA & Committee on Data for Science and Technology \\
\hline CPA & cryoprotective agent \\
\hline CPU & central processing unit \\
\hline CSD & ITL Computer Security Division \\
\hline $\mathrm{CT}$ & combinatorial testing \\
\hline CT & computed tomography \\
\hline CVSS & Common Vulnerability Scoring System \\
\hline $\mathrm{CY}$ & calendar year \\
\hline DARPA & DOD Defense Advanced Research Projects Agency \\
\hline DLMF & Digital Library of Mathematical Functions \\
\hline DNA & deoxyribonucleic acid \\
\hline DOC & Department of Commerce \\
\hline DOD & U.S. Department of Defense \\
\hline DOE & U.S. Department of Energy \\
\hline DOM & Document Object Model \\
\hline DPD & dissipative particle dynamics \\
\hline ECG & electrocardiogram \\
\hline EL & NIST Engineering Laboratory \\
\hline EM & expectation maximization \\
\hline ESPCI & École Supérieure de Physique et Chimie Industrielles de la Ville de Paris (France) \\
\hline FASTER & Faster Administration of Science and Technology Education and Research \\
\hline FDA & Food and Drug Administration \\
\hline FEM & finite element method \\
\hline FFT & fast Fourier transform \\
\hline FY & fiscal year \\
\hline $\mathrm{F} 90 \mathrm{gl}$ & Fortran 90 interface to OpenGL graphics standard \\
\hline GAC & Geodesic Active Contour \\
\hline GAMS & Guide to Available Mathematical Software \\
\hline GPU & graphics processing units \\
\hline
\end{tabular}




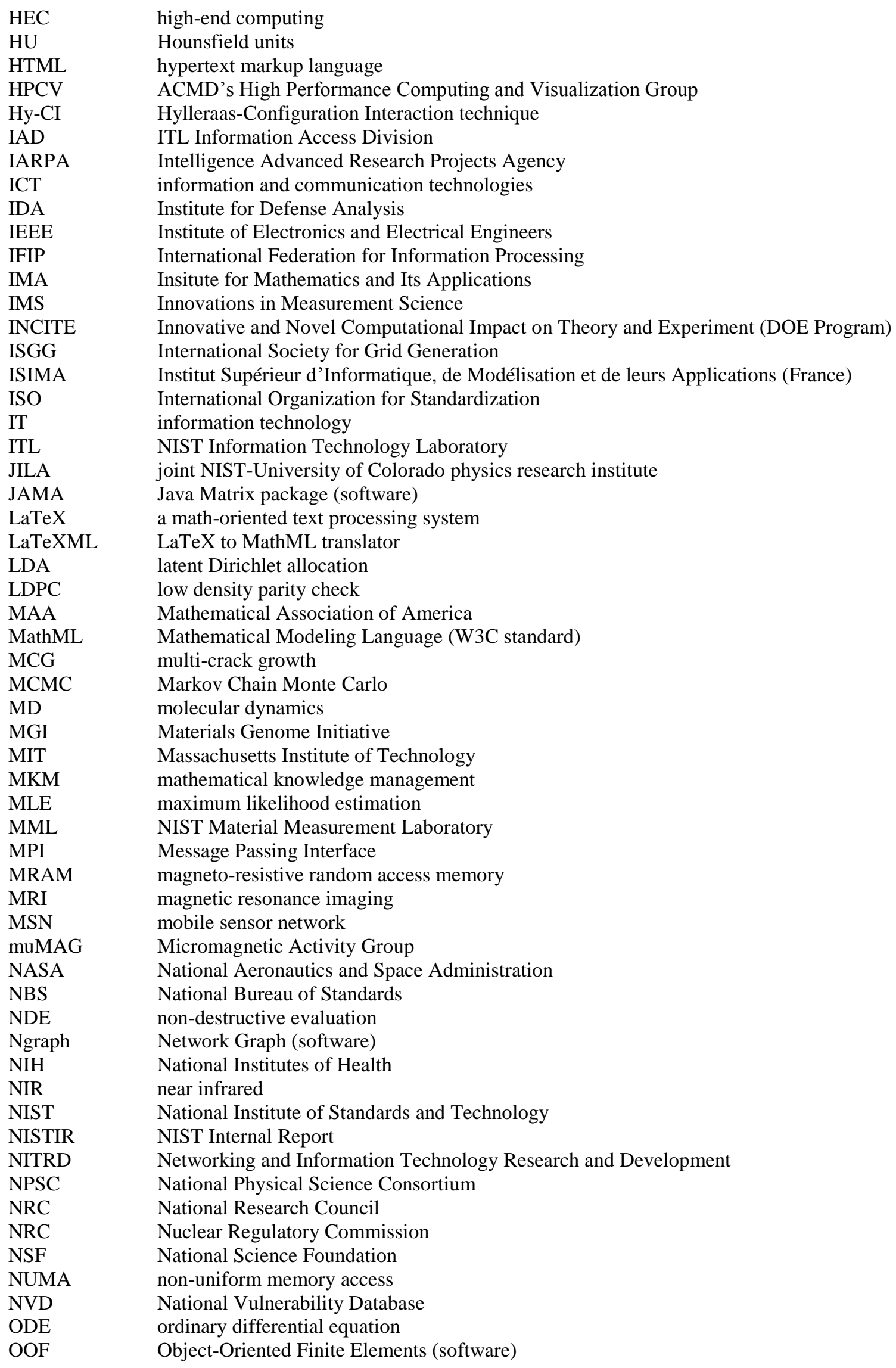




\begin{tabular}{ll} 
OOMMF & Object-Oriented Micromagnetic Modeling Framework (software) \\
PDE & partial differential equation \\
PHAML & Parallel Hierarchical Adaptive Multi Level (software) \\
PML & NIST Physical Measurement Laboratory \\
PREP & Professional Research Experience Program \\
PSNR & peak signal-to-noise ratio \\
QDPD & quarternion-based dissipative particle dynamics \\
QIBA & Quantitative Imaging Biomarkers Alliance \\
QIS & quantum information science \\
QKD & quantum key distribution \\
R\&D & research and development \\
REGEN & software for modeling of cryocoolers \\
RF & radio frequency \\
SAR & specific absorption rate \\
SBIR & Small Business Innovative Research \\
SCO & NIST Standards Coordination Office \\
SED & ITL Statistical Engineering Division \\
SHIP & NIST Summer High School Internship Program \\
SIAM & Society for Industrial and Applied Mathematics \\
SIGGRAPH & ACM Special Interest Group on Graphics \\
SparseLib++ & software for spare linear algebra in C++ \\
SPIE & International Society for Optical Engineering \\
SRM & standard reference material \\
SSD & ITL Software and Systems Division \\
STEP & NIST Student Temporary Employment Program \\
SUNY & State University of New York \\
SURF & Student Undergraduate Research Fellowship \\
SVG & scalable vector graphics \\
TCIA & The Cancer Imaging Archive \\
TNT & Template Numerical Toolkit (software) \\
TPAC & W3C Technical Plenary Advisory Committee \\
TV & total variation \\
UK & United Kingdom \\
UMBC & University of Maryland Baltimore County \\
UWB & ultra-wide band \\
VRML & virtual reality modeling language \\
W3C & World Wide Web Consortium \\
WAS & Washington Academy of Sciences \\
WebGL & Web-based Graphics Library \\
X3D & Extensible 3D \\
X3DOM & an open-source frameword for integrating X3D and HTML5 \\
XML & Extensible Markup Language \\
\hline
\end{tabular}

